\title{
The adolescent brain : neurocognitive development and subject-related factors
}

Citation for published version (APA):

Keulers, E. H. H. (2010). The adolescent brain : neurocognitive development and subject-related factors. [Doctoral Thesis, Maastricht University]. Neuropsych Publisher. https://doi.org/10.26481/dis.20101203ek

Document status and date:

Published: 01/01/2010

DOI:

10.26481/dis.20101203ek

Document Version:

Publisher's PDF, also known as Version of record

\section{Please check the document version of this publication:}

- A submitted manuscript is the version of the article upon submission and before peer-review. There can be important differences between the submitted version and the official published version of record.

People interested in the research are advised to contact the author for the final version of the publication, or visit the DOI to the publisher's website.

- The final author version and the galley proof are versions of the publication after peer review.

- The final published version features the final layout of the paper including the volume, issue and page numbers.

Link to publication

\footnotetext{
General rights rights.

- You may freely distribute the URL identifying the publication in the public portal. please follow below link for the End User Agreement:

www.umlib.nl/taverne-license

Take down policy

If you believe that this document breaches copyright please contact us at:

repository@maastrichtuniversity.nl

providing details and we will investigate your claim.
}

Copyright and moral rights for the publications made accessible in the public portal are retained by the authors and/or other copyright owners and it is a condition of accessing publications that users recognise and abide by the legal requirements associated with these

- Users may download and print one copy of any publication from the public portal for the purpose of private study or research.

- You may not further distribute the material or use it for any profit-making activity or commercial gain

If the publication is distributed under the terms of Article $25 \mathrm{fa}$ of the Dutch Copyright Act, indicated by the "Taverne" license above, 


\section{The adolescent brain:}

Neurocognitive development and

subject-related factors 
(C) Esther H.H. Keulers, Maastricht 2010

Cover Michel Lemaire (www.lemaire-artwork.com)

Production Datawyse/Universitaire Pers Maastricht

Publisher Neuropsych Publisher

ISBN 978-94-6159-010-7 


\title{
The adolescent brain: Neurocognitive development and subject-related factors
}

\author{
Proefschrift \\ ter verkrijging van de graad van doctor aan de Universiteit Maastricht, \\ op gezag van de Rector Magnificus, Prof. mr. G.P.M.F. Mols, \\ volgens het besluit van het College van Decanen, \\ in het openbaar te verdedigen \\ op vrijdag 3 december 2010 om 10:00 uur \\ door \\ Esther Hendrika Hubertina Keulers \\ geboren 5 november 1980 te Geleen
}

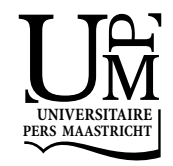




\section{Promotor}

Prof. dr. J. Jolles (Vrije Universiteit Amsterdam/Universiteit Maastricht)

Copromotor

Dr. P. Stiers

Beoordelingscommissie

Prof. dr. H.W.M. Steinbusch (voorzitter)

Dr. S. Durston (Universitair Medisch Centrum Utrecht)

Dr. E. Formisano

Prof. dr. A.M. Korebrits (Radboud Universiteit Nijmegen/Universiteit Maastricht)

Em. Prof. dr. J. Troost

Prof. dr. H.B.M. Uylings (VU Universitair Medisch Centrum Amsterdam)

The research described in this thesis was performed at the department of Psychiatry and Neuropsychology, School for Mental Health and Neuroscience, Maastricht University, Maastricht, the Netherlands.

Financial support for the publication of this thesis was kindly provided by Lundbeck B.V. and Nutricia Advanced Medical Nutrition. 


\section{$C_{\text {oreme }}$}

$\begin{array}{lll}\text { Chapter } 1 \quad \text { Introduction } & 7\end{array}$

$\begin{array}{lll}\text { Chapter } 2 & \text { Age, sex and pubertal phase influence mentalizing } & 17\end{array}$ about emotions and actions in adolescents

Chapter 3 Developmental changes between ages 13 and 21 years in the extent and magnitude of the BOLD response during decision making

Chapter 4 Decoding age from task-induced brain activation in adolescence

Chapter 5 Developmental fMRI studies: should cortisol reactivity 103 be considered as confounder?

Chapter $6 \quad$ Sex differences in brain activation are related to motivational context rather than to the nature of decision making

Chapter $7 \quad$ Concluding remarks

Summary

Samenvatting

Curriculum Vitae

Publications 

Chapter $\perp$

Introduction 


\section{Scope of thesis}

Across cultures and centuries, adolescence has been described as a period of profound changes in body and behavior situated between child and adulthood. Despite developmental gains in the cognitive domain (e.g. Huizinga et al., 2006; Luna et al., 2004), this age period is also characterized by increased impulsive and risk taking behavior that can lead to maladaptive outcomes such as substance abuse and higher mortality rates (Casey et al., 2008; Dahl, 2004; Steinberg, 2008a). These behavioral characteristics suggest suboptimal decision making abilities, especially with respect to long term consequences of behavior. Ironically, it is particularly in this developmental period that a strong appeal is made on decision making abilities. Important decisions have to be made, ranging from decisions which have consequences for the short term, such as choosing between making homework or hanging out with friends, to choices with long term consequences, such as the selection of a continuing education. The vulnerability of adolescents is further evidenced by an increased incidence of various psychiatric disorders, such as depression and schizophrenia, in this age period (Kessler et al., 2005; Paus et al., 2008).

These striking characteristics of adolescence have in the past years increasingly been explained by prolonged maturational changes in the structure and organization of the adolescent brain. Although it is assumed that behavioral as well as cognitive changes are accompanied by age-related changes in brain activation, the effects of structural maturation on the functioning of the brain are still unclear. Therefore, the main objective of this thesis is to gain more insight into the age-related changes in brain activation underlying neurocognitive development in adolescence. More insight into these brain-behavior mechanisms might improve our understanding of the striking features of adolescence.

\section{Background}

Structural brain maturation and neurocognitive development

The first indication for extended structural brain development after childhood came from post-mortem research (Huttenlocher, 1979; Huttenlocher et al., 1983). These studies showed changes in the frontal cortex during adolescence, namely continued myelination (Yakovlev \& Lecours, 1967) as well as cortical density changes, characterized by an increase of synapses at the onset of puberty followed by elimination and reorganization of these synaptic connections (Bourgeois et al., 1994; Huttenlocher, 1979). The development of neuroimaging techniques, such as Magnetic Resonance Imaging (MRI), has made it possible to 
study brain maturation in vivo. Neuroimaging studies have provided further evidence for the extended trajectory of structural brain development, with maturational changes throughout adolescence and even into young adulthood (Giedd et al., 2009; Gogtay et al., 2004; Sowell et al., 2001). Volumes of white matter and the integrity of white matter fibers increase linearly with age (Giedd, 2004; Giorgio et al., 2010). In addition, grey matter volumes show region-specific, inverted U-shaped developmental trajectories with peak values in adolescence (Gogtay et al., 2004). These structural changes are assumed to reflect maturational processes such as myelination and synaptic pruning. Furthermore, maturation related changes in functional connectivity have been demonstrated within brain networks involved in neurocognitive functions (Fair et al., 2007, 2008; Kelly et al., 2009).

Parallel to this structural brain maturation, socio-emotional and cognitive abilities also continue to develop throughout adolescence (e.g. Choudhury et al., 2006; Huizinga et al., 2006). Differential developmental trajectories have been shown for these functions. Performance on simple aspects of cognition, such as response inhibition, reaches adult levels in early adolescence (Klenberg et al., 2001; Luna et al., 2004; Paus, 2005). Conversely, performance on more complex aspects of cognition, such as working memory manipulation and decision making, reaches adult levels in middle and late adolescence (Hooper et al., 2004; Luciana et al., 2005). In addition, socio-emotional functions, such as sensation seeking behavior, show non-linear developmental changes with a peak in mid adolescence, that have been associated with the hormonal changes related to puberty (Martin et al., 2002; Steinberg, 2007; Steinberg et al., 2008b).

\section{Functional brain maturation}

It is assumed that these neurocognitive improvements are accompanied by agerelated changes in brain activation, which can be studied by using functional MRI. Some fMRI studies revealed an increase or even the emergence of activation with age in task-related brain areas. These results suggest that areas specifically involved in executing a neurocognitive function, are stronger activated with increasing age (e.g. Brown et al., 2005; Bunge et al., 2002; Crone et al., 2006; Rubia et al., 2006). However, other fMRI studies showed a decreased magnitude of activation with increasing age in areas that are involved in task execution. These latter findings suggest that children activate more widespread task-relevant areas compared with adults, possibly reflecting the fact that children have to invest more cognitive effort in successful performance of the same task (e.g. Booth et al., 2003; Casey et al., 1997; Galvan et al., 2006). Next to this inconsistency in age-related functional changes, the reported age effects are rather subtle, as they often fail to reach significance when recommended corrections for multiple comparisons are applied (Bennett et al., 2009; Poldrack \& 
Mumford, 2009). Thus, the implications of structural brain development for brain functioning are, at present, still unclear.

Influence of subject-related and methodological confounders

The weak and inconsistent findings regarding functional changes underlying neurocognitive development might suffer from contamination with the confounding influence of subject-related and methodological factors. First, uncontrolled subject characteristics might confound developmental findings. Namely, a specific age, sex and pubertal phase have been shown to affect behavioral performance as well as the brain activation patterns during neurocognitive tasks (e.g. Christakou et al., 2009; Steinberg et al., 2008b). For example, differential trajectories of structural brain development in male and female adolescents have been found (e.g. Lenroot \& Giedd, 2010), as well as sex differences in various neurocognitive functions (Bolla et al., 2004; Garavan et al., 2006). Consequently, including both male and female subjects in a developmental study increases intersubject variability and decreases fMRI sensitivity, leading to weaker age group differences. Second, developmental fMRI findings might be confounded by methodological factors, which seem to be related to age. Namely, the amount of head motion during scanning, variability in anatomy and functional organization as well as stress reactivity to fMRI scanning might be higher in children and adolescents compared with adults (Berl et al., 2006; Eatough et al., 2009; Velanova et al., 2008). For example, head motion causes signal intensity changes due to inhomogeneities in the magnetic field, which can not be explained by the task (Friston et al., 1996; Johnstone et al., 2006). Consequently, a higher amount of head motion in younger age groups reduces the signal to noise ratio in these groups, giving rise to reduced activation. Thus, to establish true age-related changes in functional activation, the role of these confounders should be investigated and controlled for.

\section{Thesis aims}

Age-related changes in brain activation underlying neurocognitive development during adolescence are still largely unknown. Furthermore, previous developmental findings might be confounded by uncontrolled subject-related and methodological factors. Therefore, the first aim of this thesis is to examine neurocognitive development in adolescence, and age-related functional changes underlying this development. The second aim is to investigate the influence of various subject-related and methodological factors on these neurocognitive developmental trajectories. Differences between male and female adolescents are investi- 
gated as the major subject-related factor, and stress-reactivity to fMRI scanning is the major methodological factor investigated.

\section{Approach and outline of thesis}

In order to pursue these thesis aims, we conducted two large-scale crosssectional studies, in which different age groups within the adolescence period were compared on both behavioral performance and brain activation during neurocognitive tasks. In addition, differences related to sex and stress reactivity were investigated. In the first study, behavioral data on several neuropsychological tasks were collected in adolescents aged 13-19. The second study included both behavioral and fMRI data of in total 86 adolescents and young adults. Methodological confounders, such as head motion and anatomical variability, were controlled for in these fMRI data. In total, the thesis consists of five chapters that report empirical studies on neurocognitive development in adolescence.

Chapter 2 describes a behavioral study in which the developmental trajectories of emotional versus cognitive functions were examined. A total of 252 adolescents divided into four age groups performed various neuropsychological tasks. In addition, the influence of two subject-related factors, i.e. sex and pubertal phase, on these developmental trajectories was investigated.

In chapter 3, we examined developmental changes in brain activation during the performance of a challenging gambling paradigm. This study aimed to evaluate the often reported developmental hypothesis of progressive focalization in functional activation from childhood to adulthood, while controlling for several confounding factors that may bias towards focalization. We compared the functional activation patterns of 13, 17 and 21 year-old male participants during the decision phase of the gambling task.

In chapter 4, an event related gonogo task was administered to three male adolescent groups of respectively 13, 17 and 21 years old. Next to the traditional, univariate voxel-wise group comparison, multivariate pattern classification analysis was introduced in order to examine whether age-related changes in brain activation were widespread throughout the brain, as indicated by maturational changes in anatomy and functional connectivity. In addition, we investigated whether this pattern of functional maturation was specific to a particular cognitive function or not, by exploring the generalizability of the classification results to different conditions of the gonogo task as well as to a totally different cognitive task.

Chapter 5 deals with cortisol changes in relation to fMRI scanning in young and old adolescents to determine whether cortisol reactivity to imaging was age- 
dependent. The association between cortisol and brain activation during a gambling task was studied as well.

In chapter 6, we examined the influence of male/female sex on brain activation during decision making tasks. Motivational context as well as the nature of decision making were manipulated in these two tasks. The functional activation patterns of male and female young adults within a small age range of 20-21 years were compared.

Finally, in chapter 7 the results described in the empirical chapters are discussed in the context of the thesis aims. In addition, theoretical implications as well as recommendations for future research are considered. 


\section{References}

Bennett, C.M., Miller, M.B., Wolford, G.L., 2009. Neural correlates of interspecies perspective taking in the post-mortem Atlantic Salmon: an argument for multiple comparisons correction. In: Organization for Human Brain Mapping Abstracts.

Berl, M.M., Vaidya, C.J., Gaillard, W.D., 2006. Functional imaging of developmental and adaptive changes in neurocognition. NeuroImage, 30, 679-691.

Bolla, K.I., Eldreth, D.A., Matochik, J.A., Cadet, J.L., 2004. Sex-related differences in a gambling task and its neurological correlates. Cerebral Cortex, 14, 1226-1232.

Booth, J.R., Burman, D.D., Meyer, J.R., Lei, Z., Trommer, B.L., Davenport, N.D., et al., 2003. Neural development of selective attention and response inhibition. NeuroImage, 20, 737-751.

Bourgeois, J.P., Goldman-Rakic, P.S., Rakic, P., 1994. Synaptogenesis in the prefrontal cortex of rhesus monkeys. Cerebral Cortex, 4, 78-96.

Brown, T.T., Lugar, H.M., Coalson, R.S., Miezin, F.M., Petersen, S.E., Schlaggar, B.L., 2005. Developmental changes in human cerebral functional organization for word generation. Cerebral Cortex, 15, 275-290.

Bunge, S.A., Dudukovic, N.M., Thomason, M.E., Vaidya, C.J., Gabrieli, J.D.E., 2002. Immature frontal lobe contributions to cognitive control in children: Evidence from fMRI. Neuron, 33, 301-311.

Casey, B.J., Getz, S., \& Galvan, A. (2008). The adolescent brain. Developmental Review, 28, 62-77.

Casey, B.J., Trainor, R.J., Orendi, J.L., Schubert, A.B., Nystrom, L.E., Giedd, J.N., et al., 1997. A developmental functional MRI study of prefrontal activation during performance of a go-no-go task. Journal of Cognitive Neuroscience, 9(6), 835-847.

Choudhury, S., Blakemore, S. J., Charman, T., 2006. Social cognitive development during adolescence. Social Cognitive and Affective Neuroscience, 1(3), 165-174.

Christakou, A., Halari, R., Smith, A.B., Ifkovits, E., Brammer, M., Rubia, K., 2009. Sex dependent age modulation of frontostriatal and temporo-parietal activation during cognitive control. NeuroImage, 48, 223-236.

Crone, E.A., Wendelkern, C., Donohue, S., van Leijenhorst, L., Bunge, S.A., 2006. Neurocognitive development of the ability to manipulate information in working memory. Proceedings of the National Academy of Sciences U.S.A., 103(24), 9315-9320.

Dahl, R.E., 2004. Adolescent brain development: a period of vulnerabilities and opportunities. Keynote address. Annals of the New York Academy of Sciences, 1021, 1-22.

Eatough, E.M., Shirtcliff, E.A., Hanson, J.L., Pollak, E.A., 2009. Hormonal reactivity to MRI scanning in adolescents. Psychoneuroendocrinology 34, 1242-1246.

Fair, D.A., Cohen, A.L., Dosenbach, N.U.F., Church, J.A., Miezin, F.M., Barch, D.M., et al., 2008. The maturing architecture of the brain's default network. Proceedings of the National Academy of Sciences U.S.A., 105(10), 4028-4032.

Fair, D.A., Dosenbach, N.U.F., Church, J.A., Cohen, A.L., Brahmbhatt, S., Miezin, F.M., et al., 2007. Development of distinct control networks through segregation and integration. Proceedings of the National Academy of Sciences U.S.A., 104, 13507-13512.

Friston, K.J., Williams, S., Howard, R., Frackowiak, R.S.J., Turner, R., 1996. Movementrelated effects in fMRI time-series. Magnetic Resonance in Medicine, 35(3), 346-355.

Galvan, A., Hare, T.A., Parra, C.E., Penn, J., Voss, H., Glover, G., et al., 2006. Earlier development of the accumbens relative to orbitofrontal cortex might underlie risk-taking behavior in adolescents. The Journal of Neuroscience, 26(25), 6885-6892. 
Garavan, H., Hester, R., Murphy, K., Fassbender, C., Kelly, C., 2006. Individual differences in the functional neuroanatomy of inhibitory control. Brain Research, 1105, 130-142.

Giedd, J.N., 2004. Structural magnetic resonance imaging of the adolescent brain. Annals of the New York Academy of Sciences, 1021, 77-85.

Giedd, J.N., Lalonde, F.M., Celano, M.J., White, S.L., Wallace, G.L., Lee, N.R. et al., 2009. Anatomical brain magnetic resonance imaging of typically developing children and adolescents. Journal of the American Academy of Child and Adolescent Psychiatry, 48(5), 465-470.

Giorgio, A., Watkins, K.E., Chadwick, M., James, S., Winmill, L., Douaud, G., et al., 2010. Longitudinal changes in grey and white matter during adolescence. NeuroImage, 49, 94103.

Gogtay, N., Giedd, J.N., Lusk, L., Hayashi, K.M., Greenstein, D., Vaituzis, A.C., et al., 2004. Dynamic mapping of human cortical development during childhood through early adulthood. Proceedings of the National Academy of Sciences U.S.A., 101(21), 81748179.

Hooper, C.J., Luciana, M., Conklin, H.M., Yarger, R.S., 2004. Adolescents' performance on the Iowa Gambling Task: implications for the development of decision making and ventromedial prefrontal cortex. Developmental Psychology, 40(6), 1148-1158.

Huizinga, M., Dolan, C. V., van der Molen, M.W., 2006. Age-related changes in executive functions: Developmental trends and a latent variable analysis. Neuropsychologia, 44, 2017-2036.

Huttenlocher, P.R., 1979. Synaptic density in human frontal cortex - developmental changes and effects of aging. Brain Research, 163, 195-205.

Huttenlocher, P.R., De Courten, C., Garey, L.J., Van Der Loos, H., 1983. Synaptic development in human cerebral cortex. International Journal of Neurology, 16-17, 144-154.

Johnstone, T., Ores Walsh, K.S., Greischar, L.L., Alexander, A.L., Fox, A.S., Davidson, R.,, et al., 2006. Motion correction and the use of motion covariates in multiple-subject fMRI analysis. Human Brain Mapping, 27, 779-788.

Kelly, A.M.C., Di Martino, A., Uddin, L.Q., Shehzad, Z., Gee1, D.G., Reiss, P.T., et al., 2009. Development of anterior cingulate functional connectivity from late childhood to early adulthood. Cerebral Cortex, 19, 640-657.

Kessler, R.C., Berglund, P., Demler, O., Jin, R., Merikangas, K.R., Walters, E.E., 2005. Lifetime prevalence and age-of-onset distributions of DSM-IV disorders in the National Comorbidity Survey Replication. Archives of General Psychiatry, 62(6), 593-602.

Klenberg, L., Korkman, M., Lahti-Nuuttila, P., 2001. Differential development of attention and executive functions in 3- to 12-year-old Finnish children. Developmental Neuropsychology, 20(1), 407-428.

Lenroot, R.K., Giedd, J.N., 2010. Sex differences in the adolescent brain. Brain and Cognition, 72(1), 46-55.

Luciana, M., Conklin, H.M., Hooper, C.J., Yarger, R.S., 2005. The development of nonverbal working memory and executive control processes in adolescents. Child Development, 76(3), 697-712.

Luna, B., Garver, K.E., Urban, T.A., Lazar, N.A., Sweeney, J.A., 2004. Maturation of cognitive processes from late childhood to adulthood. Child Development, 75(5), 1357-1372.

Martin, C.A., Kelly, T.H., Rayens, M.K., Brogli, B.R., Brenzel, A., Smith, W.J., et al., 2002. Sensation seeking, puberty and nicotine, alcohol and marijuana use in adolescence. Journal of the American Academy of Child and Adolescent Psychiatry, 41, 1495-1502.

Paus, T., 2005. Mapping brain maturation and cognitive development during adolescence. Trends in Cognitive Sciences, 9, 60-68. 
Paus, T., Keshavan, M., Giedd, J.N., 2008. Why do many psychiatric disorders emerge during adolescence? Nature Reviews Neuroscience, 9, 947-957.

Poldrack, R.A., Mumford, J.A., 2009. Independence in ROI analysis: where is the voodoo? Social Cognitive and Affective Neuroscience, 4, 208-213.

Rubia, K., Smith, A.B., Woolley, J., Nosarti, C., Heyman, I., Taylor, E., et al., 2006. Progressive increase of frontostriatal brain activation from childhood to adulthood during event-related tasks of cognitive control. Human Brain Mapping, 27, 973-993.

Sowell, E.R., Thompson, P.M., Tessner, K.D., Toga, A.W., 2001. Mapping continued braingrowth and gray matter density reduction in dorsal frontal cortex: Inverse relationships during postadolescent brain maturation. The Journal of Neuroscience, 21(22), 88198829.

Steinberg, L., 2007. Risk taking in adolescence: New perspectives from brain and behavioral science. Current Directions in Psychological Science, 16, 55-59.

Steinberg, L., 2008a. A social neuroscience perspective on adolescent risk-taking. Developmental Review, 28, 78-106.

Steinberg, L., Albert, D., Cauffman, E., Banich, M., Graham, S., Woolard, J., 2008b. Age differences in sensation seeking and impulsivity as indexed by behavior and self report: Evidence for a dual systems model. Developmental Psychology, 44(6), 1764-1778.

Velanova, K., Wheeler, M.E., Luna, B., 2008. Maturational changes in anterior cingulate and frontoparietal recruitment support the development of error processing and inhibitory control. Cerebral Cortex, 18, 2505-2522.

Yakovlev, P.A., Lecours, I.R., 1967. The myelogenetic cycles of regional maturation of the brain. In A. Minkowski (Ed.), Regional development of the brain in early life (pp. 370). Oxford: Blackwell. 

Chapter

\section{Age, sex and pubertal phase influence mentalizing about emotions and actions in adolescents}

Esther H.H. Keulers, Elisabeth A.T. Evers, Peter Stiers and Jelle Jolles Developmental Neuropsychology, 35(5), 555-569. 


\begin{abstract}
This study examined 1) emotional versus cognitive developmental trajectories and 2) the influence of age-extrinsic factors, i.e. sex and puberty. Using a crosssectional design, adolescents $(\mathrm{N}=252)$ divided into four age-groups (ages $13,15,17,19)$ performed two versions of a mentalizing task, about emotions and actions, as well as the Tower task. Firstly, performance on all tasks improved linearly into late adolescence (age 19). Thus no differential trajectories were found for emotional versus cognitive development. Secondly, girls outperformed boys in mentalizing speed regarding both emotions and actions. In boys, a later pubertal phase was associated with increased mentalizing speed after controlling for age-group.
\end{abstract}




\section{Introduction}

Adolescence is a social-psychological construct that refers to the transition period between childhood and adulthood. This period is characterized by profound physical (e.g. strength), cognitive (e.g. inhibitory control) and social (e.g. independence) development. Despite these developmental gains, adolescence is characterized by suboptimal decisions and actions which become noticeable in heightened substance abuse and mortality rates (Casey et al., 2008; Dahl, 2004; Steinberg, 2008). This apparent enigma about adolescence has been explained by differential trajectories of neural development for emotional processes on the one hand, and cognitive processes on the other hand (Casey et al., 2008; Steinberg, 2005, 2007). Models explaining this enigma (Casey et al., 2008; Steinberg, 2008) are based upon the notion that limbic structures such as the striatum, which are associated with emotional processes, develop earlier than parts of the prefrontal cortex such as the dorsolateral prefrontal cortex, which underlie top-down cognitive control processes (Gogtay et al., 2004). The biological imbalance between the two systems raises consequently difficulties in the control of behavior and emotion that is typical in adolescence (Casey et al., 2008; Steinberg, 2005).

Recent brain research has indeed shown that structural brain development continues throughout adolescence and into young adulthood. This is especially the case for higher-order association areas in the prefrontal and superior temporal cortices (Giedd, 2004; Gogtay et al., 2004). Complex cognitive functions mediated by these areas are thought to be prone to late maturation. Although developmental trajectories for different cognitive functions throughout childhood have been described thoroughly (Anderson, 2002; Klenberg et al., 2001), it is only recently that the interest in neurocognitive development over the period of adolescence has arisen (Huizinga et al., 2006; Luna et al., 2004). Consequently, little is known as yet about the neurodevelopmental trajectories of emotional in comparison with cognitive functions in adolescence. It has been hypothesized that these developmental trajectories are different because the underlying neural substrates of emotional processes mature earlier than those of cognitive control (Gogtay et al., 2004; Steinberg, 2007). The present study was devised to test this hypothesis: a cross-sectional design was used with four age groups of respectively 13, 15, 17 and 19 years. An additional research question pertained to the influence of sex on these developmental trajectories. This question was based upon the evidence that the brain of boys and girls matures at different rates (Giedd et al., 2006).

The present study is based on Steinberg's model (2007), which distinguishes two networks controlled by different neural processes namely a 'socio- 
emotional' and a 'cognitive control' network. The socio-emotional network, which is involved in processing social and emotional information, is assumed to mature under the influence of hormonal changes related to puberty (Nelson et al., 2005; Steinberg, 2007). Puberty refers to the physical changes that mark the beginning of sexual maturation. Some studies (Diamond et al., 1983; McGivern et al., 2002) suggested that the socio-emotional maturation shows a discontinuous pattern. For example, McGivern and colleagues (2002) demonstrated a slower reaction time in children of pubertal age compared with both younger and older children on a match-to-sample task in which participants had to match emotional faces to emotion words. However, Choudhury and colleagues (2006) found linear improvement in the performance on a mentalizing task in which participants had to answer questions regarding how they or another person would feel in various scenarios. These two studies were limited by the fact that they failed to measure pubertal phase quantitatively. So, the developmental trajectory of emotional processes remains largely unknown. The cognitive control network, which controls complex executive functions such as planning and selfregulation, is thought to develop linearly with age and over a longer time period than the socio-emotional network (Steinberg, 2007). Behavioral performance on cognitive control tasks such as response inhibition, working memory and decision-making did indeed appear to improve throughout adolescence. They reached adult levels of performance at ages between 14 and 19 years (Hooper et al., 2004; Luciana et al., 2005; Luna et al., 2004). Nevertheless, this prolonged maturation throughout adolescence was not found for other cognitive functions such as cognitive flexibility and goal-setting (Anderson et al., 2001). In the present study, we used an experimental mentalizing task including both an emotional and cognitive version (Maastricht Mentalizing Task, MMT). The MMT is comparable with previous used mentalizing tasks (Berthoz et al., 2002; Blakemore et al., 2007; Choudhury et al., 2006; Gallagher et al., 2000). Functional MRI studies using these mentalizing tasks showed that mentalizing areas such as medial prefrontal cortex, superior temporal sulcus and the temporal poles were activated (Blakemore et al., 2007; Gallagher et al., 2000). This proved the validity of such a task paradigm, we used here as well. We evolved this task in an attempt to study emotional and cognitive control processes in a comparable task paradigm, which in addition is ecologically valid. The MMT is a computerized task in which participants have to imagine real-life situations and make decisions. Mentalizing is defined as the ability to attribute, understand and think about mental states, such as intentions, beliefs and desires, of oneself and others in order to predict the resulting behavior (Fonagy et al., 2002; Frith \& Frith, 2003). The first version, MMT-emotion, involved the evaluation of an emotional situation from either a first or third person perspective. The MMT-emotion was used as a measure of the socio-emotional network in Steinberg's model (2007). The second version, MMT-action, requested participants to evaluate the conse- 
quences of events or behavior. The MMT-action was used as a measure of the cognitive control network in Steinberg's model (2007). Additionally, the Tower task of the Delis-Kaplan Executive Function System battery (D-KEFS; Delis et al., 2001a) was administered. The Tower task measured executive functions such as spatial planning. It was used as a more traditional measure of the cognitive control network (Steinberg, 2007).

Along with the absence of quantitative measures of pubertal phase, discrepancies between above-mentioned developmental studies may also reflect a lack of experimental control over inter-individual differences. Age-extrinsic factors, such as sex and level of parental education, are important to consider as they are associated with cognitive development. Sex is known to influence the rate of structural brain development as girls reach peak grey matter thickness a few years earlier than boys (Blanton et al., 2004; De Bellis et al., 2001; Giedd et al., 2006). On the behavioral level, some studies showed a superior performance by girls on verbal tasks and tasks involving empathy and theory of mind, whereas boys were superior on spatial tasks especially on mental rotation (Baron-Cohen, 2002; Burman et al., 2008; Linn \& Petersen, 1985; Wassenberg et al., 2008). However, these sex differences have not been consistently found (Anderson, 2002; Clark et al., 2006). Another important factor is the level of parental education. Higher levels were associated with higher performance on verbal and executive functioning tasks (Ardila et al., 2005; Hoff, 2003; Klenberg et al., 2001). Finally, the neuropsychological changes accompanying puberty are a third factor worth studying. This is because changing sex hormones during puberty have been associated with changes in the limbic system. These result in changes in arousal and emotions as well as increased sensation seeking and risk-taking behavior (Martin et al., 2002; Nelson et al., 2005). Until recently, the effect of puberty on tasks measuring cognitive control has received limited attention (McGivern et al., 2002). Moreover, reliable measures of pubertal development are often lacking in adolescent research (Dorn et al., 2006). Because the different pubertal phases were all represented in the boys in our sample, the present study examined as a minor research question the influence of pubertal phase on both emotional and cognitive functions in boys. Unfortunately, the influence of puberty could not be examined in girls in the present study, as the majority of girls were already postpubertal within the age range studied here. In this study we investigated the influence of two age-extrinsic factors, i.e. sex and puberty in boys, on the mentalizing and Tower tasks, as these factors are assumed to influence the developmental trajectories.

In sum, the aim of the present study was twofold. First of all, the developmental trajectories of the socio-emotional in comparison with the cognitive control network throughout adolescence were examined. The mentalizing task which involved emotional situations (MMT-emotion) was used as a measure of socio-emotional processes; whereas both the mentalizing task evaluating conse- 
quences of events or behavior (MMT-action) and the D-KEFS Tower task were used as measures of cognitive processes. We hypothesized that the development on the MMT-emotion would be complete prior to the MMT-action and Tower task. Secondly, the influence on these tasks of two age-extrinsic factors, i.e. sex and puberty in boys, was studied. The level of parental education was controlled for. Sex-specific developmental trajectories were expected for both the socioemotional and cognitive control functions. Furthermore, in boys the pubertal phase was hypothesized to primarily influence socio-emotional functioning as measured with the MMT-emotion.

\section{Method}

\section{Participants}

Participants were recruited through advertisements and information talks from secondary schools and a university, in the southern region of the Netherlands. Participants received an information package, containing a letter about the purpose of the study, an informed consent form and a short questionnaire about demographic, developmental and medical variables. In the case of participants aged 17 or less, informed consent was also obtained from the parents. Due to summer holidays, the data were collected in two waves, with the first wave in May-June 2007 and the second wave in October-December 2007. Participants in the seventh (13/14 year-olds), ninth (15/16 year-olds) and eleventh (17/18 yearolds) grades of pre-university education and in the first year of university (19/20 year-olds) were recruited in the first wave. In order to incorporate similar mean ages in the second wave, participants of the same cohort who had not yet participated were approached in the next school year. All participants were free from developmental, psychiatric or neurological abnormalities. They had never repeated or skipped a grade and did not use medication that could influence cognitive functioning (such as methylphenidate); those using medication for asthma were included.

A total of 252 participants (121 boys and 131 girls) were included of which $46.0 \%$ in the first wave. These participants were split up into four age groups as follows: group $1(\mathrm{~N}=70$, mean age $=13.34, \mathrm{SD}=0.40)$, group $2(\mathrm{~N}=67$, mean age $=15.33, \mathrm{SD}=0.33)$, group $3(\mathrm{~N}=58$, mean age $=17.38, \mathrm{SD}=0.39)$ and group 4 $(\mathrm{N}=57$, mean age $=19.62, \mathrm{SD}=0.51)$. The proportion of boys and girls did not differ between age groups $\left(\chi_{(3)}^{2}=1.42 ; p=.701\right)$. Level of Parental Education (LPE) was based on the parent with the highest level of education, measured with a commonly-used Dutch education scale (Directoraat-Generaal voor Arbeidsvoorziening, 1989; Kalff et al., 2001). One parent-pair (0.4\%) had a low level of parental education, 79 parent-pairs (31.3\%) had a moderate level, and 
172 (68.3\%) parent-pairs had a high level of education. Because only 1 parentpair had a low level of education, level of parental education was scored dichotomously: middle (elementary school to general secondary education) versus high (high vocational education to doctoral degree). Level of parental education differed between age groups $\left(\chi_{(3)}^{2}=11.69 ; \mathrm{p}=.009\right)$. The percentage of parents with a middle education was lower in group 4 compared with the three other groups (respectively $\mathrm{p}=.001$ versus group $1 ; \mathrm{p}=.009$ versus group 2 and $\mathrm{p}=.011$ versus group 3). Therefore, LPE was included as a covariate in further analyses.

\section{Procedure}

All participants were tested individually in a separate, quiet room. Testing was performed by well trained researchers or research assistants. Administration of the test battery took approximately 90 minutes. Tests were taken in the same order and consisted of two versions of the mentalizing task (MMT), three subtests of the Delis-Kaplan Executive Function System (D-KEFS; Delis et al., 2001a), and a computerised questionnaire about school and daily functioning. Only data from the two versions of the mentalizing task and the D-KEFS Tower task are presented in the present paper. The study was approved by the ethical committee of the Faculty Psychology and Neuroscience of the Maastricht University.

\section{Instruments}

The Maastricht Mentalizing Task (MMT) was used to measure the speed of mentalizing processes and decision-making about real-life situations. Participants were presented with hypothetical situations on a computer screen, followed by a question. They were instructed to imagine the situation, think of a possible answer and press the spacebar when they had done so. They had 15 seconds to respond, after which the task automatically continued. The elapsed time was taken as the dependent variable. If participants did not respond within the maximal period of 15 seconds, thinking time was valued as this maximum time (incidence $=0.12 \%$ of all MMT items). An exception was made for participants who did not respond to preceding and/or following questions as well. These cases may be caused by distraction or confusion and therefore these trials were excluded (incidence $=0.07 \%$ of all MMT items). Furthermore, thinking times below 1 second were considered unreliable and were excluded as well (incidence $=0.06 \%$ of all MMT items). The two versions of the MMT, MMTemotion and MMT-action, both consisted of 72 items, randomly presented to the participants. Item examples of both MMT versions will be given in the next two paragraphs. Between items a fixation cross was visible for the mean duration of 2 seconds, fluctuating between 0.5 and 3.5 seconds. The number of characters used to describe the hypothetical situation and question were kept between 45 
and 55 characters. The MMT was programmed in E-prime (Version 1.1; Psychology Software Tools, 2002).

The MMT-emotion required participants to think about emotional situations, from either a first (36 items) or a third (36 items) person perspective. After the participants thought about the hypothetical situation and pressed the spacebar, they had to indicate their answer by pressing either the '1 (Yes)' or '5 (No)' button on the keyboard. This answer screen was maximally visible for $1.5 \mathrm{sec}$ onds and disappeared if the participant pressed ' 1 ' or ' 5 '. An example of a first person perspective, the first item category, is: -hypothetical situation and question- 'During a play you forget your lines. Do you feel embarrassed? -followed by answer- Yes/ No'. An example of a third person perspective, the second item category, is: -hypothetical situation and question- 'Your mother sees a rat in the kitchen. Will she be afraid? -followed by answer- Yes/ No'.

The MMT-action included two item categories, namely items related to causal consequences of actions or behavior (36 items) and items related to planning own behavior and its consequences (36 items). After the participants thought about the hypothetical situation and pressed the spacebar, the computer gave a possible answer followed by Yes/ No. Participants were asked to indicate on a keyboard whether (press ' 1 ') or not (press '5') the computer's answer matched their own answer. This answer screen was maximally visible for 2.5 seconds and disappeared if the participant pressed ' 1 ' or ' 5 '. This time is longer than in the MMT-emotion, because in the MMT-action participants additionally had to compare their answer with that of the computer. An example item related to causal consequences is: -hypothetical situation and question- 'You forget to water the flowers. What will happen then?-followed by possible answer- The flowers wither. Yes/ No'. An example item related to behavioral planning is: hypothetical situation and question- 'You miss the train for an important appointment. What do you do? -followed by possible answer- You cancel the appointment. Yes/ No’.

The Delis-Kaplan Executive Function System - Tower task (D-KEFS; Delis et al., 2001a) was used to measure spatial planning ability. The Tower task is typically used to assess executive functions such as planning, rule learning, inhibition of impulsive responding, as well as visual-perceptual abilities (Delis et al., 2001b). The task required the movement of 5 different-sized disks across three pegs in order to duplicate the goal configuration. Participants had to transform the starting state into the goal state in the fewest number of moves possible while following two rules: 1) only one disk may be moved at a time and 2) a larger disk may not be placed on top of a smaller disk. The task consisted of 9 items of increasing difficulty consisting of 3 two-disks, 2 three-disks, 2 fourdisks and 2 five-disks items. The 9 items required respectively 1, 2, 3, 4, 7, 9, 13, 20 and 26 moves. For this study, three parameters were used as dependent variables, namely the first-move time (average time to make a first move across 
all correctly completed items), the mean move time (total time/ total number of moves across all correctly solved items) and accuracy (total number of correctly solved items).

The self-report version of the Pubertal Developmental Scale (PDS; Carskadon \& Acebo, 1993, the originally English version was translated into Dutch) was used to measure pubertal status. Good reliability and validity data of this scale have been reported (Bond et al., 2006; Petersen et al., 1988). Furthermore, pubertal self-reports correlated highly with physician ratings using Tanner stages (Carskadon \& Acebo, 1993). Questions included in the scale concerned growth spurt, body hair growth and skin changes. Additionally, sex-specific questions assessed breast growth and menarche for girls, and voice change and facial hair growth for boys. Items were rated on a 4-points scale, ranging from 1 (maturation not started) to 4 (maturation completed). Menarche was scored dichotomously as yes (4 points) or no (1 point). Total scores on 3 questions (for boys: body hair growth, voice change, facial hair growth; for girls: body hair growth, breast development and menarche) were recorded in five stages: 1) prepubertal, 2) early pubertal, 3) midpubertal, 4) late pubertal and 5) postpubertal, according to Crokett and colleagues (1988 in: Carskadon \& Acebo, 1993). For the present study these five stages were recoded into three stages, prepubertal $(1,2)$, pubertal $(3)$ and postpubertal $(4,5)$, in order to get an equal distribution of the number of participants over all stages.

\section{Statistical analysis}

The statistical package SPSS 16.0 was used for all analyses. Before statistical analyses, logarithmic transformations were conducted on all thinking and reaction time variables to meet the criteria for normality. First of all, developmental patterns and the influence of sex were determined. For the two MMT-versions, an analysis of covariance (ANCOVA) was conducted with age group (4 levels) and sex (2 levels) as between-subject variables, item category (4 levels) as within-subject variable, and LPE (2 levels) as a covariate. For first-move time and mean move time on the Tower task, an ANCOVA was used with age group (4 levels) and sex (2 levels) as between-subject variables, speed measure (2 levels) as within-subject variable, and LPE (2 levels) as a covariate. Accuracy on the Tower task was analysed with a non-parametric Kruskal-Walis test due to non-normality. If statistically significant effects were found, post hoc comparisons were performed. Additionally, polynomial trend analyses were performed for all dependent thinking and reaction time variables to determine whether the developmental patterns showed a linear and/or a quadratic course. The alpha value was set at .017 for these analyses after Bonferonni correction.

Secondly, the effect of pubertal phase on mentalizing was tested in a subsample that included only boys from group 1 and 2. This sub-sample was se- 
lected because of the specific age window in which there was enough variation in pubertal scores and a reflection of all pubertal phases. Girls were excluded because they barely showed variation in pubertal phase, as the majority of girls were already postpubertal at the time of assessment. The percentage of postpubertal girls in the 4 age groups was respectively 61.8\%, 100\%, 97.1\% and 100\%. Because pubertal phase is strongly related to age group, both variables were included in the analyses. The influence of pubertal phase on the two MMTversions was examined with an ANCOVA with pubertal phase (3 levels) as between-subject variable, item category (4 levels) as within-subject variable, and age group (2 levels) as a covariate. If statistically significant effects were found, post hoc comparisons were performed. Additionally, polynomial trend analyses were performed for all variables to determine whether puberty had a linear and/or a quadratic influence. The alpha value was set at .05 for the puberty analyses.

\section{Results}

Developmental patterns and sex differences

In case of the MMT participants were excluded from further analyses if they had not pressed the spacebar 4 or more times, as a consequence of which the thinking time had to be filled in with the maximum 15 seconds for these items. Five participants (1.98\%) were excluded from MMT analyses.

With regard to the MMT, a significant main effect for age group ( $\mathrm{F}_{(3}$, 239) $=5.65 ; \mathrm{p}=.001)$, sex $\left(\mathrm{F}_{(3,239)}=9.34 ; \mathrm{p}=.003\right)$ and item category were revealed $\left(\mathrm{F}_{(3,239)}=11.66 ; \mathrm{p}<.001\right.$; Figures 1 and 2$)$. The interaction effect age group $\mathrm{x}$ item category was not significant $\left(\mathrm{F}_{(3,239)}=1.50 ; \mathrm{p}=.144\right)$, suggesting that the developmental trajectories of the MMT-emotion and MMT-action did not differ. Post hoc comparisons showed that the oldest age group mentalized faster than the youngest age group $(\mathrm{p}<.001)$ and also a trend for faster mentalizing compared with age group 2 was found $(p=.021)$. The trend analysis confirmed these results by showing a linearly decreasing trend over age groups for mentalizing speed for emotions related to both self $\left(\mathrm{F}_{(3,239)}=31.39 ; \mathrm{p}<.001\right)$ and others $\left(\mathrm{F}_{(3 \text {, }}\right.$ 239) $=26.62 ; \mathrm{p}<.001)$ and for items related to causal consequences $\left(\mathrm{F}_{(3,239)}=7.73\right.$; $\mathrm{p}=.006)$ and to behavioral planning $\left(\mathrm{F}_{(3,239)}=6.31 ; \mathrm{p}=.013\right)$. The quadratic trend was not significant for any of the item categories. Additionally, boys needed more time to mentalize about both emotional situations and consequences of events and behavior than girls $(\mathrm{p}=.003)$. Furthermore, the mean thinking time for items related to behavioral planning was the longest (respectively $\mathrm{p}<.001$ versus first person emotional items, $\mathrm{p}<.001$ versus third person emotional items, and $\mathrm{p}<.001$ versus causal consequences items), followed by thinking time for items 




Figure 1: Mean thinking time on MMT-emotion with error bars indicating standard error of the mean

related to causal consequences (respectively $\mathrm{p}<.001$ versus first person emotional items and $\mathrm{p}=.001$ versus third person emotional items). In addition, thinking time for emotional items requiring a third person perspective were longer compared with items requiring a first person perspective $(p<.001)$. Neither LPE nor any other interaction term was significant.

In the case of the D-KEFS Tower task ten participants were excluded who were familiar with and trained in the Tower or similar tests due to computer games. Additionally, extreme outliers with a mean score of 3 standard deviations below or above the total mean were excluded from further analyses to meet the criteria for normality. In total, eighteen participants (7.14\%) were excluded 


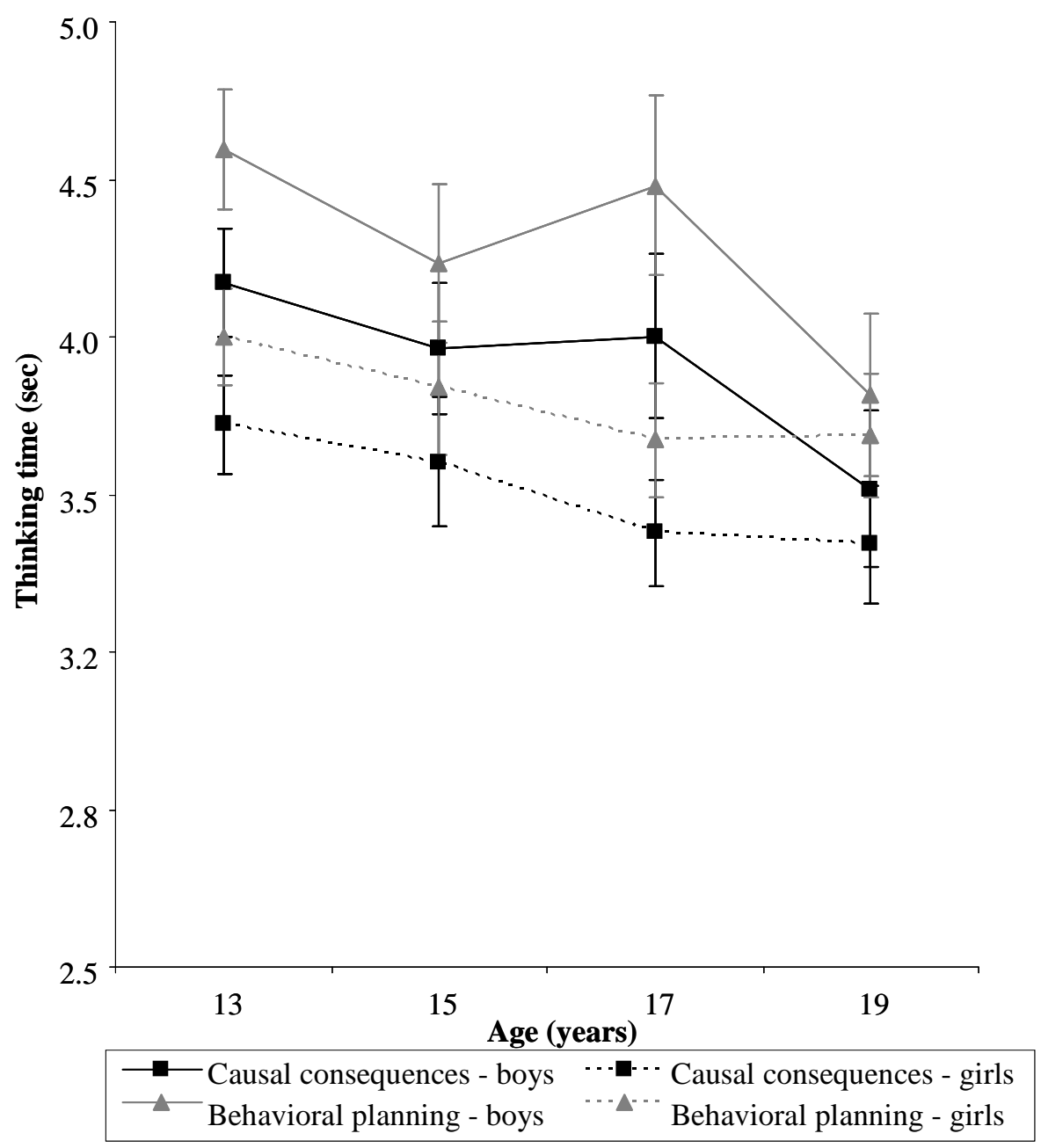

Figure 2: Mean thinking time on MMT-action with error bars indicating standard error of the mean

for first-move time and mean move time, and ten (3.97\%) for accuracy on the DKEFS Tower task.

With regard to the move time on the D-KEFS Tower task, the ANCOVA showed a significant age group $\mathrm{x}$ speed measure interaction effect $\left(\mathrm{F}_{(3,222)}=\right.$ 6.95; $\mathrm{p}<.001)$. Posthoc analyses revealed a main effect for age group on the firstmove time $\left(\mathrm{F}_{(3,222)}=5.91 ; \mathrm{p}=.001\right)$ but not on mean move time $\left(\mathrm{F}_{(3,222)}=2.61\right.$; $\mathrm{p}=.053$ ). The oldest age group thought longer before they made their first move than the three younger age groups (respectively $\mathrm{p}=.001$ versus group $1 ; \mathrm{p}=.011$ versus group 2 and $\mathrm{p}=.006$ versus group 3 ), which did not differ from each 
other. The trend analysis showed a linear $\left(\mathrm{F}_{(3,222)}=12.04 ; \mathrm{p}=.001\right)$ trend for firstmove time over age groups. Trend analysis for mean move time showed no significant results. Additionally, the ANCOVA and trend analysis were repeated separately for easy (item 1 to 4 ) and difficult (items 5 to 9 ) items. For the easy items both the ANCOVA and trend analysis revealed no significant age group effects. For the difficult items the age group $x$ speed measure interaction effect was confirmed $\left(\mathrm{F}_{(3,22)}=6.64 ; \mathrm{p}<.001\right)$ with a main effect for age group $\left(\mathrm{F}_{(3,}\right.$ 222) $=7.19 ; \mathrm{p}<.001)$ and a linear trend over age groups $\left(\mathrm{F}_{(3,222)}=14.60 ; \mathrm{p}<.001\right)$ for the first-move time. Furthermore, neither sex, nor LPE or any other interaction term was significant.

Accuracy did not differ between age groups $\left(\chi_{(3)}^{2}=2.63 ; p=.453\right)$, so groups did not differ in the number of items of the Tower task they solved correctly. The results remained unchanged also when analyses were repeated separately for easy (item 1 to 4) and difficult (items 5 to 9) items.

Influence of pubertal phase

The sub-sample used for the puberty analyses consisted of 69 male participants from age group 1 and 2, distributed over the three puberty stages: prepubertal $(\mathrm{N}=25)$, pubertal $(\mathrm{N}=28)$ and postpubertal $(\mathrm{N}=16)$. The distribution of pubertal stages was different for the two age groups $\left(\chi_{(1)}^{2}=48.88 ; \mathrm{p}<.001\right)$. Within age group 1 the majority of participants were prepubertal or pubertal, whereas within age group 2 the majority of participants were pubertal or postpubertal. Consistent with this, there was a positive correlation between pubertal stage and age group ( $\mathrm{r}=.737 ; \mathrm{p}<.001)$. To estimate the independent contribution of puberty on thinking times, both pubertal phase and age group were included in the ANCOVA.

The ANCOVA for the MMT revealed a main effect for pubertal stage $\left(\mathrm{F}_{(1}\right.$, 67) $3.18 ; \mathrm{p}=.048$ ). Post hoc analyses showed that participants in the prepubertal phase needed more time to think of an answer than participants in the postpubertal phase $(\mathrm{p}=.042)$. Additionally, trend analysis showed a linear decrease in thinking time over increasing pubertal phases for emotional items requiring a first person perspective $\left(\mathrm{F}_{(1,67)}=5.44 ; \mathrm{p}=.023\right)$, for items related to causal consequences $\left(\mathrm{F}_{(1,67)}=4.81 ; \mathrm{p}=.032\right)$ and to behavioral planning $\left(\mathrm{F}_{(1,67)}=7.09\right.$; $\mathrm{p}=.010)$. The trend analysis for emotional items requiring a third person perspective $\left(F_{(1,67)}=2.08 ; p=.154\right)$ as well as the effect of age group $\left(F_{(1,67)}=1.40\right.$; $\mathrm{p}=.241$ ) were not significant. 


\section{Discussion}

The present findings showed that both emotional and cognitive processes develop further through late adolescence, as assessed with an experimental mentalizing task (MMT-emotion and MMT-action) and the D-KEFS Tower task. However, emotional and cognitive processes did not show differential developmental trajectories. Furthermore, age-extrinsic factors proved to be of importance in these developmental patterns. Girls outperformed boys in mentalizing speed with regard to both emotion and action, but they did not outperform boys on the Tower task. In boys, a later pubertal phase was associated with increased mentalizing speed.

The first aim of the present study was to examine developmental trajectories of emotional in comparison with cognitive processes. We found no evidence for differential developmental trajectories as hypothesized based on theoretical models (Casey et al., 2008; Steinberg, 2008). Thus, the hypothesized earlier maturation of emotional in comparison with cognitive control processes was not confirmed for the ability to evaluate emotional situations from either a first or a third person perspective as measured here. However, differential developmental trajectories might still play a role in other emotional implementations compared with cognitive control as shown in other studies (Galvan et al., 2006; Gardner \& Steinberg, 2005). Gardner and Steinberg (2005) revealed that risk taking of adolescents is more influenced by the presence of peers than that of adults and Galvan and colleagues (2006) showed enhanced nucleus accumbens activity but a less mature response in prefrontal control regions during reward in adolescents compared with adults. In both cases, adolescents are compared with adults more sensitive to socio-emotional arousal (e.g. peers, reward) at the expense of the regulatory effectiveness of the still immature cognitive control network. The discrepancy between these and the present findings can be explained by differences in the implementation of emotional processes. In the present study we used virtual situations in which emotional situations versus consequences of behavior and events had to be evaluated in a laboratory environment, whereas the two other studies manipulated the real socio-emotional environment by including the presence of peers or different reward magnitudes to the tasks (Galvan et al., 2006; Gardner \& Steinberg, 2005).

The speed of mentalizing for both emotional items and items regarding consequences of events and behavior increased linearly with age. Regarding the MMT-emotion, adolescents were able to make faster assessments of emotional situations from both a first and a third person perspective with increasing age. Overall faster responses were recorded for first compared with third person perspectives, independent of age group. Another study on emotional mentalizing did find that the reaction time difference between first and third person perspectives decreased with age, which indicates more efficient perspective taking with 
increasing age (Choudhury et al., 2006). This change might still occur outside the age range measured in the present study, as is suggested by other studies which used an older adult group, viz. between ages 21 and 24 (Choudhury et al., 2006; Heatherton et al., 2006; Ruby \& Decety, 2004). Participants in the present study were instructed to imagine the emotional situation before they answered. This contrasts with the study of Choudhury and colleagues (2006), in which the instruction was to answer as quickly as possible. This difference in task instruction could also explain the different findings regarding perspective taking. Studies that used another measure of emotional processes, for example a face processing task, also showed improvement throughout adolescence, although the course was varying linear and quadratic (McGivern et al., 2002; Thomas et al., 2007). Future research should further investigate the specific course of emotional development throughout adolescence. Regarding the MMT-action, adolescents needed less time to mentalize about the consequences of actions and planned behavior with increasing age. Additionally, more time was needed by participants to mentalize about items related to planning their own behavior and its consequences compared with items related to the causal consequences of actions or behavior. This difference might be caused by a discrepancy in cognitive processing for the two item categories of the MMT-action. Items concerning planning behavior and its consequences (for instance going to the supermarket, missing a train, needing a new bike) might leave more answer options open to consideration than items concerning causal consequences of events and behavior, which are more straightforward (for instance oversleeping, sales, forgetting to water flowers). Blakemore and colleagues (2007) did not find behavioral differences between adolescents and adults on their mentalizing task. However, the tasks differ substantially on two points in the task design. First, in the present study open questions were used as opposed to closed questions by Blakemore and colleagues (2007). Second, this study is based on the thinking time taken between the hypothetical situation screen and key press for the end of the thinking process; whereas Blakemore and colleagues (2007) used the reaction time taken as the time between the answer screen and the key press for the answer. We opted for thinking time instead of real reaction speed to imitate real-life situations and measure cognitive processes such as working memory, weighing pros and cons, and selecting one behavior option. The MMT could be improved for future research in order to optimize the direct parallel between the emotion and action version by using the same situations, the exact same task design and timing and comparable item categories.

With respect to the D-KEFS Tower task, the present findings suggested that only one measure improved into late adolescence, namely the planning time needed to make the first move. Accuracy and mean move times already stabilized in early adolescence viz. at ages 12-13. The oldest age group thus seemed to use a different strategy and approach to solve these spatial problems com- 
pared with the three younger age groups, by thinking longer before making the first move. This difference in planning time was caused by the difficult items of the Tower task, as there were no developmental differences on the easy items. However, this different strategy did not result in a better accuracy score. The present findings corresponded to previous research, with most studies showing stabilization in adolescence viz. at ages 14-15, for dependent variables of the Tower task such as number of moves and time to solve the items (Asato et al., 2006; De Luca et al., 2003; Huizinga et al., 2006). The number of Tower items solved in the minimum number of moves was found to improve into late adolescence until age 21 (Huizinga et al., 2006). However, all three above-mentioned studies used the Tower of London task, which consists of three balls and less number of moves needed to solve an item than the Tower version used in the present study, which consisted of five disks. The more difficult Tower version used in the present study was able to show the prolonged development of the first-move time into late adolescence. This finding showed that developmental differences in adolescence might be subtle, but can be detected using sensitive and more complex tasks.

The second aim of this study was concerned with the influence of two ageextrinsic factors on emotional and cognitive development in order to explain inter-individual variance. With respect to the influence of the first age-extrinsic factor, i.e. sex, we found that boys thought longer than girls on both MMT versions but did not differ on the Tower measures. Boys needed more time than girls to evaluate and judge hypothetical real-life situations, with regard to both emotions and the consequences of events or planned behavior. The advantage of girls on the MMT-emotion is in line with the literature about social skills and decision-making (Baron-Cohen, 2002; Carroll \& Chiew, 2006; D’Acremont \& Van der Linden, 2006). Furthermore, these data match previous findings of girls outperforming boys on verbal tasks, for the MMT contained a large verbal component (Burman et al., 2008; Wassenberg et al., 2008). On the Tower task a superior performance by boys might be expected, given the spatial nature of this task (Clark et al., 2006). However, this sex effect was absent in the present study. Similarly, sex has been noted as a non-significant factor in Tower performance in other developmental studies (De Luca et al., 2003; Luciana \& Nelson, 2002).

The second age-extrinsic factor considered here, i.e. puberty in boys, did influence the thinking time on the MMT after correcting for age group. More specifically, the speed of mentalizing with regard to MMT-action items and to emotional items requiring a first person perspective increased linearly over increasing pubertal phases and was faster in postpubertal participants compared with prepubertal ones. These findings confirm the claim based on theoretical models about the importance of puberty within the age-window of adolescence (Dahl, 2004; Dorn et al., 2006; Steinberg, 2007). In spite of the expected effects 
of pubertal phase on emotional processes, we found puberty influences on both emotional and cognitive mentalizing items. The linear influence of pubertal phase on mentalizing speed found in the present study did not match earlier studies, which found a non-linear influence of puberty (Diamond et al., 1983; McGivern et al., 2002). However, the study by McGivern and colleagues (2002) inferred puberty from the chronological age of participants instead of measuring puberty quantitatively. Therefore the present study, which used a self-report version of the Pubertal Developmental Scale, is in a better position to evaluate the influence of puberty. Because we studied the influence of puberty only in boys, future research should extend this research question to larger samples of both boys and girls.

In all main analyses the level of parental education was controlled for as the percentage of high educated parents was higher in group 4 compared with the three other age groups. Level of parental education did not influence any of our dependent variables. Therefore, it is unlikely that our results have been affected by the unequal distribution of parental education over age groups. The absence of an association between level of parental education and cognition as suggested by others (e.g. Klenberg et al., 2001) might be due to a relatively high mean for parental education in this study. Future research should include more adolescents with a lower level of education to further examine this issue.

In conclusion, the large cross-sectional study we conducted has indicated still ongoing development into late adolescence with regard to mentalizing about emotions and about the consequences of events and planned behavior, as well as with regard to spatial planning. Performance on all three tasks showed a linear developmental pattern throughout adolescence. Our findings did not confirm the differential developmental trajectories as hypothesized on the one hand for emotional processes measured with the MMT-emotion, and on the other hand for cognitive processes measured with the MMT-action and the Tower task (Casey et al., 2008; Steinberg, 2007). Age-extrinsic factors proved to be of importance. Sex influenced mentalizing speed concerning real-life situations, with girls outperforming boys. Moreover, puberty emerged as an important factor to consider in adolescent research, as pubertal phase had an effect on mentalizing abilities in boys aged 12-15 after controlling for age group. These findings may have implications for educational practice and pedagogical interventions as they suggest that a) emotional and cognitive capacities continue to develop into late adolescence though the changes might be subtle and b) sex and puberty are important age-extrinsic factors to consider in adolescents' development. 


\section{References}

Anderson, P., 2002. Assessment and development of executive function (EF) during childhood. Child Neuropsychology, 8, 71-82.

Anderson, V., Anderson, P., Northam, E., Jacobs, R., Catroppa, C., 2001. Development of executive functions through late childhood and adolescence in an Australian Sample. Developmental Neuropsychology, 20, 385-406.

Ardila, A., Rosselli, M., Matute, E., Guajardo, S., 2005. The influence of the parents' educational level on the development of executive functions. Developmental Neuropsychology, 28(1), 539-560.

Asato, M.R., Sweeney, J.A., Luna, B., 2006. Cognitive processes in the development of TOLperformance. Neuropsychologia, 44, 2259-2269.

Baron-Cohen, S., 2002. The extreme male brain theory of autism. Trends in Cognitive Sciences, 6(6), 248-254.

Berthoz, S., Armony, J.L., Blair, R.J.R., Dolan, R.J., 2002. An fMRI study of intentional and unintentional (embarrassing) violations of social norms. Brain, 125, 1696-1708.

Blakemore, S.J., den Ouden, H., Choudhury, S., Frith, C., 2007. Adolescent development of the neural circuitry for thinking about intentions. Social Cognitive and Affective Neuroscience, 2(2), 130-139.

Blanton, R.E., Levitt, J.G., Peterson, J.R., Fadale, D., Sporty, M. L., Lee, M., et al., 2004. Gender differences in the left inferior frontal gyrus in normal children. Neuroimage, 22(2), 626-636.

Bond, L., Clements, J., Bertali, N., Evans-Whipp, T., McMorris, B. J., Patton, G.C., et al., 2006. A comparison of self-reported puberty using the Pubertal Development Scale and the Sexual Maturation Scale in a school-based epidemiological study. Journal of Adolescence, 29, 709-720.

Burman, D.B., Bitan, T., Booth, J.R., 2008. Sex differences in neural processing of language among children. Neuropsychologia, 46(5), 1349-1362.

Carroll J.M., Chiew K.Y., 2006. Sex and discipline differences in empathising, systemising and autistic symptomatology: evidence from a student population. Journal of Autism and Developmental Disorders, 36, 949-957.

Carskadon, M.A., Acebo, C., 1993. A self-administered rating scale for pubertal development. Journal of Adolescent Health, 14, 190-195.

Casey, B.J., Getz, S., Galvan, A., 2008. The adolescent brain. Developmental Review, 28, 6277.

Choudhury, S., Blakemore, S.J., Charman, T., 2006. Social cognitive development during adolescence. Social Cognitive and Affective Neuroscience, 1(3), 165-174.

Clark, C.R., Paul, R.H., Williams, L.M., Arns, M., Fallahpour, K., Handmer, C., et al., 2006. Standardized assessment of cognitive functioning during development and aging using an automated touchscreen battery. Archives of Clinical Neuropsychology, 21(5), 449467.

D’Acremont, M., van der Linden, M., 2006. Gender differences in two decision-making tasks in a community sample of adolescents. International Journal of Behavioral Development, 30, 352-358.

Dahl, R.E., 2004. Adolescent brain development: a period of vulnerabilities and opportunities. Keynote address. Annals of the New York Academy of Sciences, 1021, 1-22. 
De Bellis, M.D., Keshavan, M.S., Beers, S.R., Hall, J., Frustaci, K., Masalehdan, A., et al., 2001. Sex differences in brain maturation during childhood and adolescence. Cerebral Cortex, 11(6), 552-557.

De Luca, C.R., Wood, S.J., Anderson, V., Buchanan J., Proffitt, T. M., Mahony, K., et al., 2003. Normative data from the CANTAB I: Development of executive function over the lifespan. Journal of Clinical and Experimental Neuropsychology, 25, 242-254.

Delis, D.C., Kaplan, E., Kramer, J.H., 2001a. Delis-Kaplan Executive Function System (DKEFS), San Antonio: The Psychological Corporation.

Delis, D.C., Kaplan, E., Kramer, J.H., 2001b. Delis-Kaplan Executive Function System (DKEFS) examiner's manual, San Antonio: The Psychological Corporation.

Diamond, R., Carey, S., Back, K.J., 1983. Genetic influences on the development of spatialskills during early adolescence. Cognition, 13, 167-185.

Directoraat-Generaal voor de Arbeidsvoorziening, 1989. Handleiding voor de functieanalyse [Function analyses manual]. Den Haag: SDU uitgeverij.

Dorn, L.D., Dahl, R.E., Woodward, H.R., Biro, F., 2006. Defining the boundaries of early adolescence: A user's guide to assessing pubertal status and pubertal timing in research with a adolescents. Applied Developmental Science, 10(1), 30-56.

Fonagy, P., Gergely, G., Jurist, E., Target, M., 2002. Affect regulation, mentalization, and the development of the self. New York: Other Press.

Frith, U., Frith, C.D., 2003. Development and neurophysiology of mentalizing. Philosophical Transactions of the Royal Society of London. Series B: Biological Sciences, 358, 45947.

Gallagher, H.L., Happe, F., Brunswick, N., Fletcher, P.C., Frith, U., Frith, C.D., 2000. Reading the mind in cartoons and stories: an fMRI study of 'theory of mind' in verbal and nonverbal tasks. Neuropsychologia, 38, 11-21.

Galvan, A., Hare, T.A., Parra, C.E., Penn, J., Voss, H., Glover, G., et al., 2006. Earlier development of the accumbens relative to orbitofrontal cortex might underlie risk-taking behavior in adolescents. The Journal of Neuroscience, 26(25), 6885-6892.

Gardner, M., Steinberg, L., 2005. Peer influence on risk taking, risk preference, and risky decision making in adolescence and adulthood: an experimental study. Developmental Psychology, 41(4), 625-635.

Giedd, J.N., 2004. Structural magnetic resonance imaging of the adolescent brain. Annals of the New York Academy of Sciences, 1021, 77-85.

Giedd, J.N., Clasen, L.S., Lenroot, R., Greenstein, D., Wallace, G.L., Ordaz, S., et al., 2006. Puberty-related influences on brain development. Molecular and Cellular Endocrinology, 256, 154-162.

Gogtay, N., Giedd, J.N., Lusk, L., Hayashi, K.M., Greenstein, D., Vaituzis, A.C., et al., 2004. Dynamic mapping of human cortical development during childhood through early adulthood. Proceedings of the National Academy of Sciences U S A, 101(21), 81748179.

Heatherton, T.F., Wyland, C.L., Macrae, C.N., Demos, K.E., Denny, B.T., Kelley, W.M., 2006. Medial prefrontal activity differentiates self from close others. Social Cognitive and Affective Neuroscience, 1(1), 18-25

Hoff, E., 2003. The specificity of environmental influence: socioeconomic status affects early vocabulary development via maternal speech. Child Development, 74(5), 1368-1378.

Hooper, C.J., Luciana, M., Conklin, H.M., Yarger, R.S., 2004. Adolescents' performance on the Iowa Gambling Task: implications for the development of decision making and ventromedial prefrontal cortex. Developmental Psychology, 40(6), 1148-1158. 
Huizinga, M., Dolan, C.V., van der Molen, M.W., 2006. Age-related changes in executive functions: Developmental trends and a latent variable analysis. Neuropsychologia, 44, 2017-2036.

Kalff, A.C., Kroes, M., Vles, J.S.H., Bosma, H., Feron, F.J.M., Hendriksen, J.G.M., et al., 2001. Factors affecting the relation between parental education as well as occupation and problem behavior in Dutch 5- to 6- year- old children. Social Psychiatry and Psychiatric Epidemiology, 36, 324-331.

Klenberg, L., Korkman, M., Lahti-Nuuttila, P., 2001. Differential development of attention and executive functions in 3- to 12-year-old Finnish children. Developmental Neuropsychology, 20(1), 407-428.

Linn, M.C., Petersen, A.C., 1985. Emergence and characterization of gender differences in spatial abilities: A meta-analysis. Child Development, 56, 1479-1498.

Luciana, M., Conklin, H.M., Hooper, C.J., Yarger, R.S., 2005. The development of nonverbal working memory and executive control processes in adolescents. Child Development, 76(3), 697-712.

Luciana, M., Nelson, C.A., 2002. Assessment of neuropsychological function through use of the Cambridge Neuropsychological Testing Automated Battery: performance in 4- to 12-year-old children. Developmental Neuropsychology, 22(3), 595-624.

Luna, B., Garver, K.E., Urban, T.A., Lazar, N.A., Sweeney, J.A., 2004. Maturation of cognitive processes from late childhood to adulthood. Child Development, 75(5), 1357-1372.

Martin, C.A., Kelly, T.H., Rayens, M.K., Brogli, B.R., Brenzel, A., Smith, W. J., et al., 2002. Sensation seeking, puberty and nicotine, alcohol and marijuana use in adolescence. Journal of the American Academy of Child and Adolescent Psychiatry, 41, 1495-1502.

McGivern, R.F., Andersen, J., Byrd, D., Mutter, K.L., Reilly, J., 2002. Cognitive efficiency on match to sample task decreases at the onset of puberty in children. Brain and Cognition, 50, 73-89.

Nelson, E.E., Leibenluft, E., McClure, E.B., Pine, D.S., 2005. The social re-orientation of adolescence: A neuroscience perspective on the process and relation to psychopathology. Psychological Medicine, 35, 163-174.

Peterson, A.C., Crockett, L., Richards, M., Boxer, A., 1988. A self-report measure of pubertal status: Reliability, Validity and Initial Norms. Journal of Youth and Adolescence, 17, 117-133.

Ruby, P., Decety, J., 2004. How would you feel versus how do you think she would feel? A neuroimaging study of perspective-taking with social emotions. Journal of Cognitive Neuroscience, 16(6), 988-999.

Steinberg, L., 2005. Cognitive and affective development in adolescence. Trends in Cognitive Sciences, 9(2), 69-74.

Steinberg, L., 2007. Risk taking in adolescence: New perspectives from brain and behavioral science. Current Directions in Psychological Science, 16, 55-59.

Steinberg, L., 2008. A social neuroscience perspective on adolescent risk-taking. Developmental Review, 28, 78-106.

Thomas, L.A., De Bellis, M.D., Graham, R., Labar, K.S., 2007. Development of emotional facial recognition in late childhood and adolescence. Developmental Science, 10, 547558.

Wassenberg, R., Hurks, P.P., Hendriksen, J.G., Feron, F.J., Meijs, C.J., Vles, J.S., et al., 2008. Age-related improvement in complex language comprehension: Results of a crosssectional study with 361 children aged 5 to 15. Journal of Clinical and Experimental Neuropsychology, 30(4), 435-448. 


\section{Chapter}



Developmental changes between ages 13 and 21 years in the extent and magnitude of the BOLD response during decision making 


\begin{abstract}
Developmental neuroimaging results have suggested a progression in focalization in functional activations from childhood to adulthood. The mechanisms underlying this process are thought to be an age-related decrease in activation extent as well as an increased magnitude in task-related areas. The present study aimed to evaluate these notions while controlling for confounders that may bias towards focalization. We used adolescent subjects in small age ranges. In addition, head motion corrections were incorporated in statistical analyses and regions of interest were identified for each participant separately to overcome inter-individual variability in anatomy and functional organization. Activation patterns of 13, 17 and 21 year-old males were compared during the decision phase of a challenging and complex gambling paradigm. The BOLD amplitude enhanced with increasing age, modulated by task conditions. First, response amplitude during difficult, endogenous relative to exogenous decisions increased with age. This decision difficulty effect was most pronounced in 21 year-olds, both in areas associated with task execution and default mode areas. Second, deciding to pass as opposed to gamble exerted more effort in inferior frontal and parietal areas only by 13 and 17 year-olds. There was neither an agerelated decrease in activation extent, nor any qualitative shifts in activated areas as suggested by the focalization hypothesis. These results suggest that although different age groups throughout adolescence engage similar brain areas during decision making, the response magnitude in these areas increases with age particularly during difficult task conditions, providing that confounding factors are controlled.
\end{abstract}




\section{Introduction}

The propensity for risk taking and sensation seeking behavior has been shown to increase during early and middle adolescence (Steinberg et al., 2008b). Poor decision making tendencies in this age group might lead to maladaptive outcomes such as increased substance abuse and mortality rates (Dahl, 2004; Paus et al., 2008; Steinberg, 2008a). This suggests that decision making and its underlying neural substrates are subject to development in this period.

Neuroimaging research has shown continued maturation and refinement of brain structures and connectivity throughout adolescence and young adulthood. Age-related changes in grey matter show region-specific, inverted U-shaped developmental trajectories assumed to reflect synaptic pruning and/or myelination. The peak of grey matter volumes is reached earliest in primary sensorimotor areas and latest in higher-order association areas such as prefrontal regions (Giedd et al., 2009; Gogtay et al., 2004). Volumes of white matter and the integrity of white matter fibers increase linearly with age, reflecting progressive myelination (Giedd, 2004; Giedd et al., 2009; Giorgio et al., 2010). Furthermore, maturational changes have been shown in functional connectivity within brain networks known to support cognitive control, such as prefrontal-parietal and anterior cingulate-insular connections (Fair et al., 2007; Kelly et al., 2009). These networks continue to specialize and reorganize throughout adolescence, with an increased functional connectivity between spatially remote cortical areas.

Parallel to this structural maturation, socio-emotional and cognitive abilities also continue to develop during adolescence (e.g. Choudhury et al., 2006; Huizinga et al., 2006; Keulers et al., 2010). Cognitive functions, which are the focus of the present study, have shown differential rates of maturation. Performance on simple aspects of cognition such as response inhibition reaches adult levels in early adolescence (Klenberg et al., 2001; Luna et al., 2004; Paus, 2005). On the other hand performance on more complex and affective aspects of cognition such as working memory and decision making reaches adult levels in middle and late adolescence (Hooper et al., 2004; Luna et al., 2004; Steinberg et al., 2008b). The relation between cognitive performance and accompanying agerelated changes in brain activity has been examined in several studies (e.g. Bunge et al., 2002; Crone et al., 2006; Rubia et al., 2006; Scherf et al., 2006; Tamm et al., 2002). From these studies a pattern of developmental changes in brain activity emerged that has been conceptualized as a shift from diffuse activation with low amplitudes to more focal activation patterns with greater amplitudes (Durston et al., 2006; Durston \& Casey, 2006). This progression towards focalization comprises two components. Firstly, the spatial extent of activation 
decreases with age. This is evidenced by a decrease or even disappearance of activation with age across a wide range of brain areas (e.g. Brown et al., 2005; Durston et al., 2006; Rubia et al., 2006; Tamm et al., 2002). These areas are hypothesized to be uncorrelated to task performance (Durston et al., 2006). Secondly, the magnitude of activation increases with age in task-relevant brain areas (e.g. Brown et al., 2005; Bunge et al., 2002; Durston et al., 2006; Rubia et al., 2006, 2007; Tamm et al., 2002). These areas are involved in the execution of a specific task in adults.

Developmental changes in the spatial extent of activation have been examined using whole brain, voxel-wise group comparison only by a few studies (Casey et al., 2002; Durston et al., 2006). Although these studies report a decrease in extent of activation with age, this actually concerns very few, small voxel clusters where the younger group had significantly more activation than the older group. For instance, Durston and colleagues (2006) found only 35 and 11 voxels, respectively, in their longitudinal and cross-sectional data. Other studies examined age-related changes in activation extent by comparing the percentage of active voxels within particular task-relevant regions of interest (ROIs) (Casey et al., 1997; Galvan et al., 2006; Luna et al., 2001; Scherf et al., 2006). The results reported are diverse and include both qualitative changes in which ROIs are recruited selectively by children or adolescents, and quantitative changes in which ROIs are recruited by all age groups but differed in the degree of activation. These quantitative age effects comprise linear decreases, linear increases as well as non-linear inverted U-shaped changes, depending on the particular ROI and study. Moreover, in some studies most of the reported agerelated changes do not survive statistical testing (Luna et al., 2001; Scherf et al., 2006). Thus, although several studies report age-related changes in activation extent, their conclusions are based on small, often not significant age effects, confined to the specific ROIs and frequently not consistent with the focalization hypothesis.

Studies examining developmental changes in the magnitude of activation in task-relevant areas have shown inconsistent results. Some studies revealed an increase or even the emergence of activation with age in areas that are involved in executing a specific task in adults (e.g. Brown et al., 2005; Bunge et al., 2002; Durston et al., 2006; Rubia et al., 2006). However, other studies showed decreased magnitude of activation with increasing age in task-relevant areas (e.g. Booth et al., 2003; Casey et al., 1997; Galvan et al., 2006). Moreover, the reported age-related effects again are subtle. The results do not seem to survive statistical significance thresholds that take into account the normally recommended corrections for multiple comparisons (Poldrack \& Mumford, 2009). Thus, evidence for increased magnitude of brain activation in task critical areas as predicted by the focalization hypothesis is inconsistent and the observed effects are rather small at best. 
The status of the focalization hypothesis is further obscured because several confounding factors stand in the way of drawing firm conclusions regarding its validity. First of all, the studies mentioned differ widely in the factor 'age'. Some studies included children (i.e. 7-12 years), adolescents (i.e. 13-17 years) and adults (i.e. 18-29 years) (Crone et al., 2006; Galvan et al., 2006; Velanova et al., 2008), whereas other studies only compared children (i.e. 8-12 years) with adults (i.e. 18-30 years) leaving the period of adolescence out of account (Bunge et al., 2002; Booth et al., 2003; van Leijenhorst et al., 2006), whereas still others compared adolescents (i.e. 9-17 years) with adults (i.e. 20-40 years) (Ernst et al., 2005; Eshel et al., 2007; Rubia et al., 2006, 2007). Adolescence is a socialpsychological construct that refers to the transition period between childhood and adulthood, although precise age ranges concerning on- and offset are lacking (Spear, 2000). However, using broad age ranges (i.e. 9-17 years) probably yield developmentally heterogeneous groups that may well bias the results towards the focalization hypothesis. After all, the contribution of structural brain development, socio-emotional changes and pubertal stage in addition to cognitive development, are highly variable (Giedd et al., 2006; Gogtay et al., 2004; Nelson et al., 2005). Consequently, the higher heterogeneity in adolescent group increases the chance of finding more diffuse and weaker activations, as has been reported by some studies (e.g. Eshel et al., 2007; Rubia et al., 2006, 2007).

Another important factor that may cloud the results is head motion. Head motion causes signal intensity changes due to inhomogeneities in the magnetic field, which are unrelated to the task (Friston et al., 1996). This unmodeled signal variation is added to the measurement error and consequently decreases the sensitivity of the fMRI analysis (Friston et al., 1996; Johnstone et al., 2006). The amount of head motion during scanning is higher in younger subjects compared with adults (Thomas et al., 1999; Velanova et al., 2008; Wenger et al., 2004). Thus, a higher amount of head motion in younger age groups reduces the signal to noise ratio in these age groups giving rise to reduced magnitude and extent of functional clusters.

Inter-individual variability in anatomical and functional organization can also have a confounding influence on developmental fMRI studies (Brett et al., 2002; Devlin \& Poldrack, 2007; Toga \& Thompson, 2007; Uylings et al., 2005). Both anatomy and functional activation patterns have been thought to be more variable in developing children (Berl et al., 2006; Gaillard et al., 2001; Wilke \& Holland, 2003). Consequently, group averaging in fMRI may lead in younger subjects to a decreased co-localization of cortical areas and consequently to reduced magnitude of activation or to a more diffuse activation pattern, thus biasing towards the focalization hypothesis (Berl et al., 2006; Manoach et al., 2000; Manoach, 2003).

A last factor that may contribute to the inconsistency of results so far is the choice of task used to study cognitive development during adolescence. Most 
studies use relatively simple cognitive tasks, although stronger age effects may be obtained when a more difficult and challenging task is used, given more prolonged developmental trajectories for the latter (e.g. Luciana et al., 2005; Luna et al., 2004). In addition, higher cognitive demands in difficult compared with simple tasks are associated with increased brain activation (Braver et al., 1997; Duncan \& Owen, 2000; Stiers et al., 2010), which may influence developmental results. Adolescence has traditionally been associated with increased risk taking and poor decision making tendencies (Dahl et al., 2004; Steinberg et al., 2008a, 2008b). Therefore, decision making in the context of a gambling task is likely to be a more effective cognitive paradigm for eliciting developmental changes in functional organization of the brain. This is confirmed by Eshel and colleagues (2007) who found an increased magnitude of activation in prefrontal and anterior cingulate cortices in adults (i.e. 20-40 years; $\mathrm{N}=16$ ) compared with adolescents (i.e. 9-17 years; $\mathrm{N}=18$ ). To our knowledge, this is the only study so far studying the decision phase of a gambling task in adolescence. So, developmental changes throughout adolescence in brain activation during the decision phase of a gambling task remain largely unknown.

In conclusion, it seems that the focalization hypothesis which has often been presented in previous fMRI studies as a possible model for development, is not strongly founded in empirical evidence as the effects reported in its favor are rather weak. In addition, it is not clear whether these weak results are merely a biasing effect of confounding variables or whether a genuine focalization effect is clouded by noise variables. Therefore, in the present study our aim was to investigate the focalization hypothesis while controlling for confounding variables. First, we used an appealing gambling task in which participants had to choose between gambling for one of the two options or passing, and could cumulate points over trials. Second, we selected three homogeneous groups of male-only participants within a small age range at three stages of adolescence into adulthood, viz. 12/13 year-olds, 16/17 year-olds and 20/21 year-olds, in order to reduce developmental heterogeneity. Third, we equated age groups on the amount of head motion and modeled sudden head movements explicitly in addition to including realignment parameters in the statistical analyses. Lastly, we quantified BOLD signal change extent and magnitude in well defined regions of interest identified within each participant's individual data analysis results and performed group comparisons on these measures. This ruled out the influence of inter-individual variability in anatomy and functional organization. Based on the focalization hypothesis we predicted that: 1) the spatial extent of activation decreased with age and 2) the magnitude of activation increased with age in task-relevant brain areas. As a measure of activation extent we established the percentage of active voxels within several brain regions delineated by masks which together covered the entire brain. Magnitude measures were subtracted for several brain areas involved in executing the present gambling task 
(Stiers et al., 2010). These ROIs corresponded with areas within recently delineated core networks that are involved in task execution regardless of specific task demands (e.g. Dosenbach et al., 2007; Duncan \& Owen, 2000; Greicius et al., 2003; Seeley et al., 2007; Sridharan et al., 2008): 1) the Central Executive Network (CEN), 2) the Salience Network (SN) and 3) the Default Mode Network (DMN).

\section{Materials and methods}

\section{Participants and procedure}

Participants in the seventh (12/13 year-olds) and eleventh (16/17 year-olds) grades of pre-university education and university students (20/21 year-olds) were recruited through advertisements and information talks. All participants had normal or corrected-to-normal vision, were free from psychiatric and neurological abnormalities, never repeated or skipped a grade, did not use medication that could influence cognitive functioning and were screened for MRI contraindications. Written informed consent was obtained from all participants and their parents in case participants aged 17 or less. Participants received travelling expenses and a fee of 25 euros. The study was approved by the ethical committee of the Faculty Psychology and Neuroscience of the Maastricht University. From the total 70 participants included in the study, 1 participant had to be excluded due to a motivational problem, 3 participants because of too few trials in one task condition (GAMBLE) and 10 participants due to excessive head motion (see section Functional Magnetic Resonance Imaging data - Preprocessing for details). This resulted in a final sample of 56 male, right-handed participants divided into three age groups as follows: 13 year-olds $(\mathrm{N}=18$, mean age $=12.93$, $\mathrm{SD}=0.34$, range 12.29-13.66), 17 year-olds $(\mathrm{N}=21$, mean age $=16.99, \mathrm{SD}=0.43$, range 16.34-17.74) and 21 year-olds $(\mathrm{N}=17$, mean age $=21.00, \mathrm{SD}=0.60$, range 20.08-21.91).

Participants attended a training session on average one week before the scan session. The training session served to familiarize participants with the scanning environment and procedure, and to practice the experimental tasks using a mock scanner. In addition, the youngest age group received a training in the mock scanner to reduce motion during scanning (Slifer et al., 1993). Participants viewed a cartoon videotape in the mock scanner for 2 x 10 minutes, which was halted whenever the participants moved their head more than $4.5 \mathrm{~mm}$. Furthermore, two neuropsychological tests were administered to obtain estimates of intelligence. Age groups did neither differ on an estimate of verbal intelligence $\left(\mathrm{M}=113.39, \mathrm{SD}=8.08, \mathrm{~F}_{(2,53)}=2.06 ; \mathrm{p}=.14\right)$ as assessed with the Peabody Picture Vocabulary Test-III-NL (Dunn \& Dunn, 2005) nor on an estimate of nonverbal 
intelligence $\left(\mathrm{M}=122.07, \mathrm{SD}=4.96, \mathrm{~F}_{(2,53)}=0.91 ; \mathrm{p}=.41\right)$ as assessed with the Raven Standard Progressive Matrices (Raven et al., 1998). The Child Behavior Checklist and the Youth Self Report were administered in the two youngest age groups (Achenbach \& Rescorla, 2001), and the Adult Self Report (Achenbach \& Rescorla, 2003) in the adults group to screen for behavioral problems. All participants had scores on the total problem scale of these questionnaires within 1 $\mathrm{SD}$ of the mean of a normative standardized sample. Furthermore, age groups did not differ on the total problem scale of the self report versions $\left(F_{(2,52)}=1.46\right.$; $\mathrm{p}=.242$ ). In addition, level of parental education was inquired with a commonlyused Dutch education scale (Directoraat-Generaal voor Arbeidsvoorziening, 1989), as higher levels of parental education have been associated with higher performance on executive functioning tasks (Ardila et al., 2005; Klenberg et al., 2001). Fifteen parent-pairs (26.8\%) had a moderate level of education (elementary school to general secondary education) and 41 (73.2\%) parent-pairs had a high level of education (high vocational education to doctoral degree). Level of parental education did not differ between age groups $\left(\chi_{(2)}^{2}=1.19 ; \mathrm{p}=.55\right)$.

Task

Participants performed an event-related gambling task in which they could decide to either gamble or pass in order to earn as many points as possible. This task was originally used in event related potentials studies (Mennes et al., 2008, 2009), but adapted by Stiers and colleagues (2010) for utilization in fMRI studies. Here we used this latter version, except for minimal changes in the timing. In each trial a horizontal bar divided into two colored parts was presented, indicating the probability of an imaginary token being hidden underneath (Figure 1). The proportion of one colored part in relation to the total bar could range from 5\%-95\% to 50\%-50\%. Participants could guess under which part a token was hidden, by pressing the corresponding left or right button. Depending on the correctness of their choice, points could be won or lost. The points that could be won were indicated by a number above the bar and varied randomly between 10 and 100 points. Below the bar a second number was presented, which indicated the points that could be lost. These were systematically linked to the proportional division of the bar, with the most ambiguous proportions (50\%-50\%) coupled with the highest losses (80-100 points). To earn as many points as possible, participants had to make gambles. However, participants could also choose to pass by withholding their response. A pass trial resulted consequently in receiving a small 20 points reward. All participants started with 100 points. In $67 \%$ of all trials, the participants received feedback about the trial and an update of their total score. The feedback could be positive ('\$\$ \$ ), negative ('too bad') or neutral after a pass trial ('?'). 


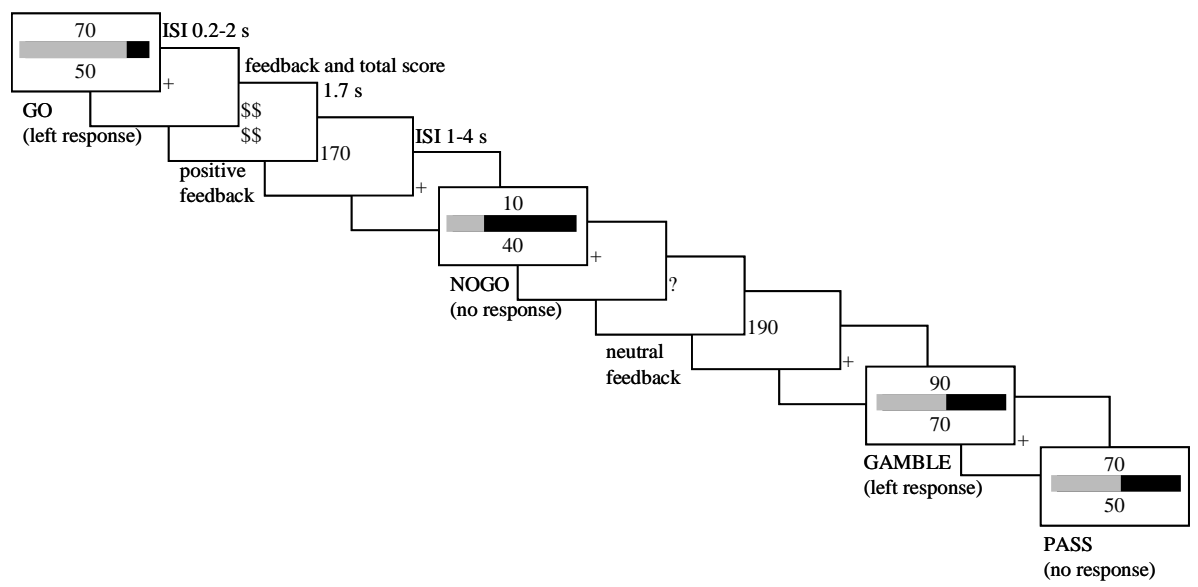

Figure 1: Stimulus sequence and timing of the gambling task. On each trial participants could decide either to gamble under which color part a token was hidden, or pass. A correct gamble was rewarded with the points above the bar, whereas an incorrect gamble resulted in loosing the point below the bar. Passing resulted in a reward of 20 points. In 2/3 of all trials, participants received feedback and an update on their total score. The first two trials in this sequence are exogenous trials in which the decision to gamble (GO) or pass (NOGO) is guided by stimulus characteristics. The last two trials are endogenous trials due to an uncertain probability of winning. The decision to gamble (GAMBLE) or pass (PASS) is guided by the participant.

The behavioral results from a previous study with this task (Mennes et al., 2008) were used to delineate four trial groups along two dimensions: 1) task difficulty: exogenous versus endogenous trials depending on whether stimulus characteristics suggested the appropriate choice or not, and 2) task response: gambling versus passing trials depending on whether a button was pressed or not. Trials characterized by a high probability of winning (80-95\%) and a high gain $(\geq 30$ points) in which participants should always gamble were exogenous gambling trials (GO). Trials characterized by a gain of 20 or less points in which participants should always pass were exogenous passing trials (NOGO). Gambling in these trials is disadvantageous as participants received a 20 points reward anyway when passing. Decisions on GO and NOGO trials were relatively easy and determined by stimulus characteristics such as the probability of winning and points that could be won. These trials are considered to measure exogenous decision making. All other trials characterized by an uncertain probability of winning (50-75\%) and a gain of 30 points or more were considered endogenous trials. Endogenous trials were more difficult as it was not immediately clear based on the stimulus characteristics which response was the most favorable. A final decision in such trials was guided by the participant and required interpretation of different kinds of information such as previous experiences, gain, 
gain/loss ratio, total score and internal motivational states. Based on whether the participant decided to gamble or pass, these endogenous trials were labeled respectively GAMBLE or PASS.

The task comprised three runs of 7.3 minutes and 50 trials each. Each trial started with the presentation of the bar for 3.5 seconds. During inter stimulus intervals a fixation cross was shown and jittering was applied to these intervals. The interval between the presentation of the bar and the start of the feedback fluctuated between 0.2 and 2.0 seconds with a mean interval of 1.1 seconds. In $2 / 3$ of the trials the participants received feedback shown for 1.7 seconds, in the remaining cases a fixation cross remained visible for this time period. The interval between the end of the feedback and the start of a new trial varied between 1.0 and 4.0 seconds with a mean interval of 2.5 seconds.

\section{Behavioral analyses}

The statistical package SPSS 16.0 was used for the analyses. Reaction times were analyzed with analysis of variance (ANOVA) with age group (3 levels) as between-subject variable and trial type (2 levels: GO vs GAMBLE) as withinsubject variable. The reaction time data did not violate the parametric assumption of ANOVA. The ratio GAMBLE/ PASS trials, indicative of a tendency to gamble, was analyzed using an oneway ANOVA with age group (3 levels) as between-subject variable. Total number of points, number of NOGO errors (i.e. failure to withhold response by a gain of 10 or 20 points) and negative feedback were analysed with a non-parametric Kruskal-Walis test due to non-normality. If statistically significant effects were found, post hoc comparisons were performed.

Functional Magnetic Resonance Imaging data

Image acquisition

A Siemens MAGNETOM Allegra 3T MRI head-only scanner was employed. Head motion was constrained by the use of foam padding. A total number of 32 axial slices covering the whole brain including the cerebellum were imaged by using a T2*-weighted gradient echo planner pulse sequence $(\mathrm{TR}=2000 \mathrm{~ms}$, TE $=30 \mathrm{~ms}, \mathrm{FA}=90, \mathrm{FOV}=224$, slice thickness $=4 \mathrm{~mm}$, matrix size $=64 \times 64$, flip angle $=90^{\circ}$ ). Voxel size was $3.5 \times 3.5 \times 4 \mathrm{~mm}$. A T1-weighted anatomical scan was acquired to aid with spatial normalization ( $\mathrm{TR}=2250 \mathrm{msec}$, $\mathrm{TE}=2.6$ msec, flip angle $=9^{\circ}$, FOV $=256 \mathrm{~mm}$, slice thickness $=1 \mathrm{~mm}$, matrix size $=256$ $\mathrm{x} 256$, number of slices $=192$ ). Voxel size was 1 x 1 x $1 \mathrm{~mm}$. Slice scanning order was ascending interleaved. 


\section{Preprocessing}

Data were preprocessed using BrainVoyager QX, version 1.9 (Brain Innovation, Maastricht, The Netherlands). Images were corrected for slice scan time differences, followed by rigid body motion correction, high-pass temporal filtering, and spatial data smoothing using a Gaussian kernel with a $4 \mathrm{~mm}$ full width at half maximum. Functional data were coregistered with the anatomical volume using rigid body transformation and manual adjustments to obtain optimal fit by visual inspection if necessary. Subsequently, both functional and structural volumes were transformed into standard stereotaxic space using Talairach normalization.

The degree of head motion during scanning was greater in the 13 year-olds compared with the two older age groups on the two most affected parameters (Mayer et al., 2007; Yoo et al., 2005), viz translation in the $\mathrm{z}$ direction (Tz; $\mathrm{F}_{(2}$, $\left.{ }_{63}=5.40 ; \mathrm{p}=.007\right)$ and rotation in the $\mathrm{x}$ direction $\left(\mathrm{Rx} ; \mathrm{F}_{(2,63)}=6.77 ; \mathrm{p}=.002\right)$. In order to control for the confounding effect of head motion we applied 3 corrections to our data. Firstly, head motion within one scan may distort the measured fMRI signal by spatial misallocation. Therefore, we identified scans during which head motion exceeded a particular threshold, viz absolute motion difference between 2 successive scans in $\mathrm{z}$ direction greater than $0.4 \mathrm{~mm}\left(1 / 10^{\text {th }}\right.$ of the voxel size), and rotation around $\mathrm{x}$ dimension greater than 0.26 degrees (angle corresponding to $0.4 \mathrm{~mm}$ z-displacement of frontopolar voxels, assuming the rotation point in middle of brain is $88 \mathrm{~mm}$ from the anterior end of the brain frontal pole (Talairach \& Tournoux, 1988)). Task trials (taking into account the hemodynamic response function delay) cause signal intensity changes with peak BOLD values 5 to 6 seconds after their occurrence. These signal changes may overlap in time with head motion a few scans later. Consequently, these signal changes are contaminated with within-scan head motion. Therefore, trials in a preceding time window of 1 to 8 seconds of the identified scan were modeled as an additional event of no interest. Because this procedure reduces the number of trials available in the events of interest, we set an upper limit of $25 \%$ to the number of trials discarded in this way. As a consequence, 4 young adolescents and 1 old adolescent were excluded because they exceeded this upper limit. After applying this procedure, age groups still differed on the motion parameters for the remaining scans, viz $\mathrm{Tz}\left(\mathrm{F}_{(2,58)}=2.81 ; \mathrm{p}=.069\right)$ and $\mathrm{Rx}\left(\mathrm{F}_{(2,58)}=6.93\right.$; $\mathrm{p}=.002)$. Therefore, as a second measure to equate age groups on the motion parameters it was unavoidable to exclude an additional 5 young adolescents with the largest head motion on the $\mathrm{Tz}$ and $\mathrm{Rx}$ parameters. This was effective in eliminating head motion differences between the 3 age groups, viz $\mathrm{Tz}\left(\mathrm{F}_{(2}\right.$, $\left.\left.{ }_{53}\right)=0.61 ; \mathrm{p}=.545\right)$ and $\mathrm{Rx}\left(\mathrm{F}_{(2,53)}=2.53 ; \mathrm{p}=.089\right)$. In total, 10 adolescents were excluded due to excessive head motion as mentioned in the participants section. Thirdly, head motion between successive scans causes signal intensity changes due to inhomogeneities in the magnetic field which are unrelated to the task 
(Friston et al., 1996). Therefore, we modeled this source of signal variance by included the 6 motion parameters as covariates of no interest in our general linear model (Friston et al., 1996; Johnstone et al., 2006).

Imaging analyses

Statistical analyses were performed on individual participants data by using the general linear model in SPM5 (Welcome Department of Cognitive Neurology, London, UK). The fMRI time series were modeled as series of events convolved with a canonical hemodynamic response function. A design matrix was set up to model all task conditions of interest (GO, NOGO, GAMBLE, and PASS trials, neutral, positive and negative feedback) and the 6 motion parameters. Error trials and trials contaminated with head motion were modeled as two separate predictors that were ignored in the further analyses.

Firstly, age group differences were examined during a global task contrast (all 4 trial types pooled > fixation) in the second level analysis of SPM5. This analysis was included to promote comparison with other developmental fMRI studies. A whole brain, voxel-wise random-effect ANOVA was used with task difficulty (2 levels: exogenous vs endogenous) and task response (2 levels: gambling vs passing) as within-subject variables and age group (3 levels) as a between-subject variable. We reported results of this whole brain analysis using a Family Wise Error (FWE<.05), False Discovery Rate (FDR<.05) error corrected level and an uncorrected level of $\mathrm{p}<.001$, all combined with a cluster threshold $\geq 5$ voxels. If statistically significant effects were found, post hoc comparisons were performed. To elaborate this analysis, the whole brain group comparison was repeated for the pooled exogenous conditions, pooled endogenous conditions and for the gamble versus pass contrast.

Secondly, we performed some analyses to specifically test the focalization hypothesis. In these analyses, we gathered brain activation measures within the single-subject analysis to rule out inter-individual variability in anatomy and functional organization and performed group comparisons on these measures in the external statistical package SPSS 16.0. To examine developmental changes in the extent of activation, we compared the percentage of task-positive and task-negative responding voxels during task performance in several brain areas. The Wake Forest University (WFU) Pick Atlas Automated Anatomical Labeling (AAL) regions were used to construct 7 main masks in both the left and right hemisphere. Some of these main masks were further divided into subregions. The frontal mask was divided into dorsolateral, inferior, orbital, medial superior, insular and motor regions. The parietal mask and the temporal mask were divided in an inferior, medial and superior region. The limbic, occipital, cerebellum and subcortical masks were not divided. The specific AAL regions used to construct each (sub)mask are described in the footnote of figure 2 (Maldjian et al., 2003; Tzourio-Mazoyer et al., 2002). These masks were constructed such 
that no overlap existed and that together they covered the entire brain. The WFU masks were transformed from MNI to Talairach space and then matched to each participants' anatomical volume using 12 parameter linear normalization in SPM5. Both a positive and a negative functional activation map per trial type (GO, NOGO, GAMBLE, PASS) was generated for each individual. A threshold of $\mathrm{p}<.001$ (corresponding $\mathrm{T}=3.25$ ) was used for these activation maps. An uncorrected threshold was used, because the FDR error correction level varies from map to map, whereas the FWE corrected level is too rigorous for individual data sets. Within each mask, the percentage of active voxels was calculated for the 8 activation maps. To determine the extent of activation independent of task condition, the mean percentage of active voxels across the 4 trial types for both the positive and negative activation maps was computed for each mask. This variable was used as the dependent variable. Due to non-normality, group comparisons were based on nonparametric randomization tests with 10,000 permutations using NPFact version 1.0 (May et al., 1993). If statistically significant effects were found, post hoc non-parametric comparisons were performed. As we directly examined the focalization hypothesis of decreased extent of activation with age, a one-tailed test of significance was used. For both task-positive and task-negative activations, the alpha value was set at .05 for each main mask after Bonferroni correction for the 2 hemispheres. If a main mask was further divided into subregions, we applied a Bonferroni correction for the number of examined subregions.

With regard to developmental changes in the magnitude of activation, we compared the Percent Signal Change (PSC) in several ROIs. To test the focalization hypothesis of increased BOLD magnitude with age in task-related areas, we chose several brain areas that are known to be involved in cognitive task performance as ROIs. Meta-analyses of fMRI studies have indicated that several brain areas play a crucial role during performance of many cognitive tasks regardless of the specific task demands (Duncan \& Owen, 2000; Mennes et al., 2006; Stiers et al., 2010). Thus, these areas are expected to be active during the present gambling task as well, which is indeed the case (Stiers et al., 2010). In addition, resting state imaging studies reinforced the idea that these areas work together in several networks: 1) the Central Executive Network (CEN; Seeley et al., 2007; Sridharan et al., 2008) which largely matches the frontoparietal network described by Dosenbach and colleagues (2007), 2) the Salience Network (SN; Seeley et al., 2007; Sridharan et al., 2008) which largely matches the cingulo-opercular network described by Dosenbach and colleagues (2007), and 3) the Default Mode Network (DMN; Dosenbach et al., 2007; Greicius et al., 2003; Thomason et al., 2008). Three positive responding ROIs were selected as part of the CEN: the Inferior Frontal Junction located at the junction of the inferior frontal and precentral sulci (IFJ; Cole \& Schneider, 2007; Derrfuss et al., 2005; Dosenbach et al., 2007), the Inferior Frontal Sulcus around its junction with the 
inferior segment of the precentral sulcus (IFS; Cole \& Schneider, 2007; Derrfuss et al., 2005; Dosenbach et al., 2007) and the IntraParietal Sulcus (IPS; Dosenbach et al., 2007; Corbetta \& Shulman, 2002). Two positive responding ROIs were selected as part of the SN: the dorsal part of the Anterior Cingulate Cortex (dACC; Amodio \& Frith, 2006; Cole \& Schneider, 2007; Dosenbach et al., 2007) and the anterior Insula (aIns; Cole \& Schneider, 2007; Derrfuss et al., 2005; Dosenbach et al., 2007). In addition, two negative responding ROIs as part of the DMN were selected: the Posterior Cingulate Cortex (PCC; Dosenbach et al., 2007; Greicius et al., 2003) and the lateral Inferior Parietal Cortex (lIPC; Dosenbach et al., 2007; Greicius et al., 2003). For guidance of identifying these ROIs we used the coordinates of these functional areas as described by Stiers and colleagues (2010). The ROIs were identified in each individual data set based on task-related signal changes in accordance with known anatomical landmarks. A global task contrast (all 4 trial types pooled $>$ fixation) was used to indentify these ROIs bilateral for each participant. The final voxels extracted were identified based on the local maximum of a functional cluster and a radius of $6 \mathrm{~mm}$. Any possible overlap between ROIs was corrected for by adjusting the radius, to maximally $4 \mathrm{~mm}$. For each ROI, the average PSC was extracted for each of the 4 trial types. Only the 20\% voxels with the highest PSC were included to make the amplitude assessments maximally independent of the extent of activation. For the CEN, SN and DMN separately, an ANOVA was conducted with age group (3 levels) as a between-subject variable and ROI (respectively 3, 2, 2 levels), laterality (2 levels), task difficulty (2 levels) and task response (2 levels) as within-subject variables. The assumption of equal error variances across groups (Levene's test) was met for all analyses. Alpha was set at .05 for each ANOVA to give maximal sensitivity. If statistically significant effects were found, post hoc comparisons were performed. Within the comprehensive ANOVAs used, we were primarily interested in developmental changes. Therefore, in the results section we will focus on main effects of age group and interactions of age group with the other factors.

Finally, we examined exploratory brain-behavior correlations as well as the correlation between extent and magnitude of activation. The mean PSC of each ROI was correlated with the activation extent within the corresponding mask. In addition, reaction times, number of NOGO errors and the GAMBLE/PASS ratio, as indications of behavioral performance, were entered into correlation analyses with PSC and activation extent. The averaged PSC across task conditions, ROIs and hemispheres was used for each brain network, i.e. CEN, SN and DMN. Task-positive and task-negative activation extent averaged across hemispheres was used for each main mask. The statistical threshold was set at $\mathrm{p}<.05$. 


\section{Results}

\section{Behavioral data}

Table 1 shows the mean and standard deviations of behavioral performance on the gambling task for the three age groups separately. The behavioral results showed neither developmental differences in impulsivity measured with reaction times $\left(\mathrm{F}_{(2,53)}=0.23 ; \mathrm{p}=.79\right)$ and NOGO errors $\left(\chi_{(2)}^{2}=4.34 ; \mathrm{p}=.11\right)$ nor developmental differences in risk-taking behavior measured with the ratio GAMBLE/ PASS trials $\left(\mathrm{F}_{(2,53)}=1.63 ; \mathrm{p}=.21\right)$, number of negative feedback $\left(\chi_{(2)}^{2}=4.80\right.$; $\mathrm{p}=.09)$ and total number of points $\left(\chi_{(2)}^{2}=1.71 ; \mathrm{p}=.43\right)$. With regard to the task conditions independent of age, the ANOVA on the reaction time data showed a main effect of trial type, with slower reaction times on GAMBLE compared with GO trials (1553.73 vs 1149.76 milliseconds; $\mathrm{F}_{(2,53)}=372.00 ; \mathrm{p}<.001$ ). This result suggests that for all age groups decision making in endogenous trials was more difficult than in exogenous trials. As task performance was comparable between age groups, the developmental findings in brain activity were unlikely to be confounded by performance differences which removed the need to control for performance in the following fMRI analyses (Berl et al., 2006; Manoach, 2003).

Table 1: Behavioral performance on the gambling task

\begin{tabular}{llll}
\hline & 13 year-olds & $\begin{array}{l}17 \text { year-olds } \\
\mathrm{N}=21\end{array}$ & $\begin{array}{l}\text { 21 year-olds } \\
\mathrm{N}=17\end{array}$ \\
\cline { 2 - 4 } RT GO trials & $\mathrm{N}=18$ & $1150.16(215.36)$ & $1096.39(192.88)$ \\
RT GAMBLE trials & $1538.97(156.90)$ & $1570.15(277.92)$ & $1549.07(290.92)$ \\
ratio GAMBLE/ PASS trials & $1.17(0.44)$ & $1.03(0.41)$ & $0.91(0.39)$ \\
total number of points & $1791.11(259.24)$ & $1877.78(211.32)$ & $1885.68(176.10)$ \\
number of NOGO errors & $0.91(0.91)$ & $0.65(0.58)$ & $0.41(0.53)$ \\
number of negative feedback & $2.78(1.21)$ & $2.01(1.26)$ & $1.96(1.33)$ \\
\hline
\end{tabular}

Note. Values are presented as mean (standard deviation). RT= Reaction Time in milliseconds.

Whole brain analysis

\section{Task activation}

We identified brain areas underlying the execution of the gambling task, both across age groups and for each age group separately. Brain activation patterns during decision making were very similar in the 3 age groups. This is illustrated in Figure 2 by drawing both positive and negative pooled task effects within each age group over one another. Positive brain activations related to decision making were found bilaterally in inferior frontal junction, dorsal part of ACC, anterior insula and intraparietal sulcus. These coincide with core functional networks involved in cognitive task execution (e.g. Dosenbach et al., 2007; Duncan 
A Positive pooled task effects

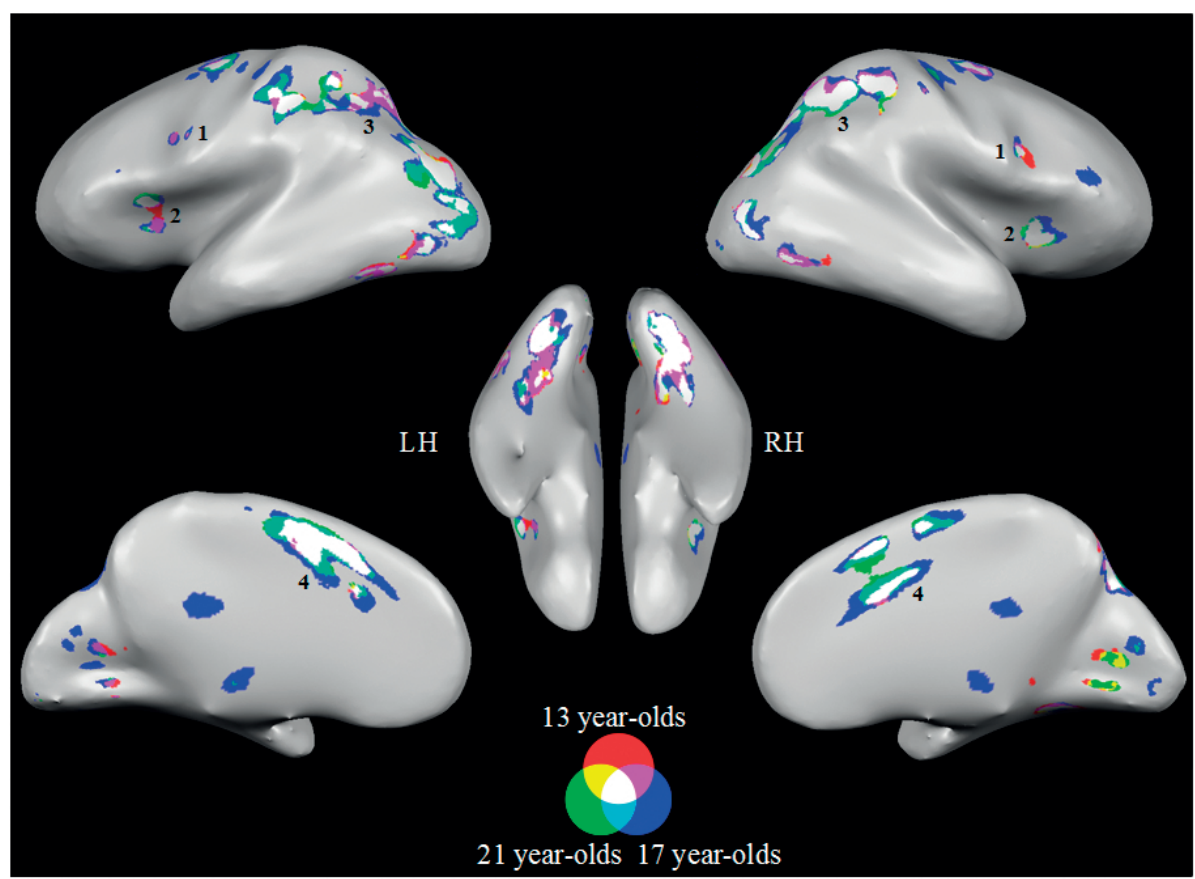

B Negative pooled task effects

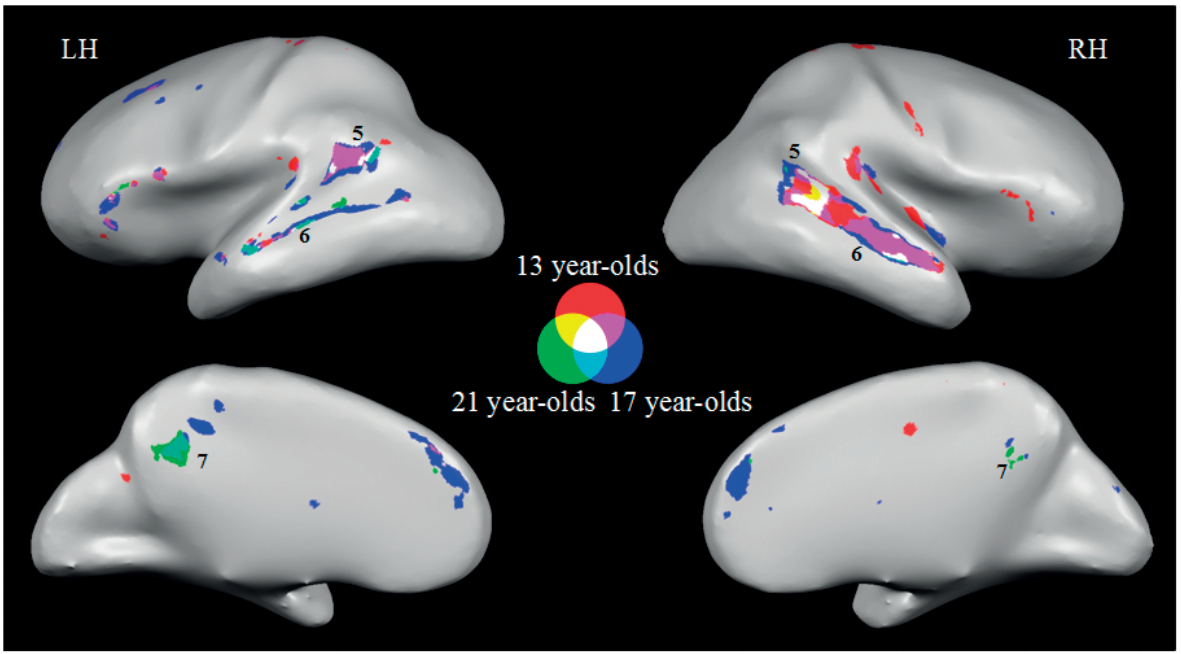

Figure 2: Functional activation patterns for the global task contrast per age group, separately for (A) positive and (B) negative activation. The functional activation pattern for the 13 yearolds is presented in red, for the 17 year-olds in bleu and for the 21 year-olds in green. The overlap in activation patterns of the 3 age groups is presented in white. Generally, the age groups (de)activated the same brain areas during the gambling task: $1=$ inferior frontal junction, $2=$ anterior insula, $3=$ intraparietal sulcus, $4=$ dorsal part of anterior cingulate cortex, $5=$ lateral inferior parietal cortex, $6=$ superior temporal gyrus, $7=$ posterior cingulate cortex. 
\& Owen, 2000; Stiers et al., 2010). Clusters in the lateral inferior parietal cortex, superior temporal gyrus and posterior cingulate cortex were deactivated during performance of the gambling task. These areas among others have been described as part of the default mode network (e.g. Dosenbach et al., 2007; Greicius et al., 2003; Stiers et al., 2010). Examination of task (de)activations across age groups yielded the same picture of brain areas involved in executing the gambling task. These findings confirm that the present gambling task was effective in modulating the BOLD signal in functional brain networks, i.e. CEN, SN and DMN.

\section{Age group differences}

The whole brain, voxel-wise analysis revealed one small cluster that significantly differed between age groups for the global task contrast at the FWE corrected level (FWE<.05; F=14.13), namely in the lingual gyrus (Talairach coordinates: 15, -81, -3). At the FDR corrected level (FDR<.05; F=10.24) which is often applied in random-effect GLM analysis, two additional clusters in the dorsal part of ACC (Talairach coordinates: 9, 15, 36) and inferior frontal sulcus (Talairach coordinates: 45, 24, 15) differed significantly between age groups. Whole brain post hoc comparisons between pairs of groups showed more activation of both lingual gyrus and dorsal part of ACC in 21 year-olds compared with 13 and 17 year-olds, who did not differ from each other. Activation of inferior frontal sulcus was higher in 17 compared with 13 as well as 21 year-olds.

Evaluation of the whole brain group comparison at the uncorrected level $(\mathrm{p}<.001 ; \mathrm{F}=7.14)$ yielded several clusters with developmental effects (Table 2). Post hoc pair-wise group analyses showed that in most of these areas, activation increased between ages 13, 17 and 21. As an exception, in inferior frontal and intraparietal sulci an inverted U-shaped developmental pattern was found. Moreover, activation of the precuneus was higher in 13 and 17 compared with 21 year-olds (Table 2).

We also looked for developmental effects in specific task conditions. These results largely coincide with the results described above for the global task contrast. At the FWE (FWE<.05; F=14.13) as well as the FDR (FDR<.05; F=10.39) corrected level the lingual gyrus (Talairach coordinates: 15, -81, -3) and dorsal part of ACC (Talairach coordinates: 9, 15, 36) again manifested small clusters of significant group difference, but only for the pooled endogenous conditions versus baseline. No other significant developmental effects were found at the FWE and FDR corrected significance levels. When the threshold was lowered to an uncorrected level of .001, an almost similar pattern of age-related changes was seen as in the global contrast (see Table 2 for details).

These whole brain analysis results indicate that adolescents activate similar brain areas as young adults during decision making. Any existing developmental changes in the age range 13 to 21 years are very small at best and do not meet 
the standard statistical requirements that are usually demanded of fMRI research. In the next section we will proceed with more specific and sensitive methods that have been used in imaging studies of development to substantiate the focalization hypothesis.

Table 2: Results of the whole brain age group comparison

\begin{tabular}{llccclllll}
\hline Brain area & Hem. & \multicolumn{9}{c}{ Talairach coördinates } & $\mathrm{F}$ & Size & Post hoc & \\
& & $\mathrm{x}$ & $\mathrm{y}$ & $\mathrm{z}$ & & & & \\
\hline dACC & $\mathrm{R}$ & 9 & 15 & 36 & 15.87 & 10 & $3>1=2$ & exo & endo \\
IFS & $\mathrm{L}$ & -48 & 21 & 27 & 14.63 & 14 & $2>1=3$ & exo & endo \\
& $\mathrm{R}$ & 45 & 24 & 15 & 12.92 & 14 & $2>1=3$ & & endo \\
SFS & $\mathrm{L}$ & -24 & -12 & 60 & 10.33 & 7 & $3>1$ & & \\
IPS & $\mathrm{L}$ & -33 & -66 & 39 & 13.60 & 9 & $2>1=3$ & & endo \\
Precuneus & $\mathrm{R}$ & 3 & -69 & 45 & 10.33 & 11 & $1=2>3$ & exo & endo \\
Lingual gyrus & $\mathrm{R}$ & 15 & -81 & -3 & 19.14 & 12 & $3>1=2$ & exo & \\
\multirow{2}{*}{ Cuneus } & & 12 & -72 & -6 & 9.67 & 9 & $2>1$ & & \\
& $\mathrm{~L}$ & -18 & -84 & 9 & 12.47 & 17 & $3>1$ & exo & endo \\
& $\mathrm{R}$ & 9 & -87 & 12 & 11.23 & 20 & $3>1=2$ & & endo \\
MOG & & 21 & -78 & 18 & 10.13 & 5 & $3>2$ & & endo \\
\hline
\end{tabular}

Note. The whole brain results for the global task contrast described here are significant at the uncorrected level $\mathrm{p}<.001$ ( $\mathrm{F}=7.14$ ) and cluster threshold $\geq 5$ voxels. Post-hoc pair-wise group comparisons are presented; $1=13$ year-olds; $2=17$ year-olds; $3=21$ year-olds. The age group differences that were confirmed for the pooled exogenous conditions and pooled endogenous conditions are shown in the last two columns. Size in number of voxels. Hem.= Hemisphere; $\mathrm{R}=$ right hemisphere; $\mathrm{L}=$ left hemisphere; exo= pooled exogenous conditions; endo= pooled endogenous conditions; dACC= dorsal part of anterior cingulate cortex; IFS= inferior frontal sulcus; $\mathrm{SFS}=$ superior frontal sulcus; IPS= intraparietal sulcus; $\mathrm{MOG}=$ middle occipital gyrus

\section{Developmental changes in the extent of activation}

First, we addressed the question whether young adolescents showed more diffuse and extended brain activation than old adolescents and adults. Therefore, we counted the number of voxels in specific brain regions that showed a significant signal change in any of the relevant task conditions, and compared these across age groups. Developmental effects for activation extent were not significant for most of the brain regions investigated. An age-related effect of taskpositive activation extent was found only for the inferior parietal cortex (alpha level .017 after Bonferroni correction for three subregions in the parietal cortex), for both the right $\left(\mathrm{F}_{(2,53)}=4.75\right.$; randomization $\left.\mathrm{p}=.011\right)$ and left $\left(\mathrm{F}_{(2,53)}=4.37\right.$; randomization $\mathrm{p}=.014$ ) hemisphere (Figure 3 ). The 13 year-olds showed less task-positive activation extent in the inferior parietal cortex compared with the 17 year-olds in both hemispheres (right randomization $\mathrm{p}=.003$; left randomization $\mathrm{p}=.006$ ) and a trend for lesser activation compared with the 21 year-olds in the left hemisphere was found (randomization $\mathrm{p}=.027$ ). In none of the investigated brain regions age groups differed in task-negative activation extent. Addi- 
tionally, repeating these analyses for task-positive and task-negative activation extent during the more difficult, endogenous task conditions yielded the same results.
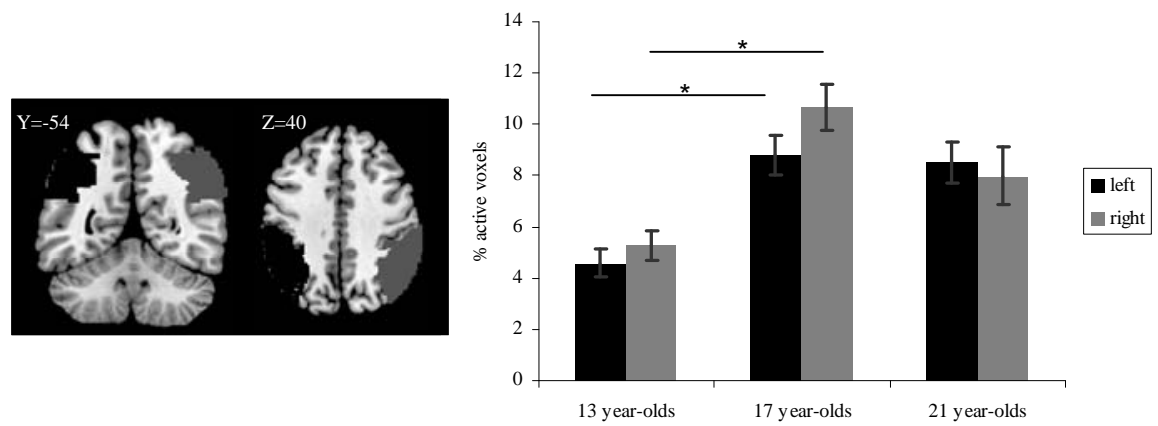

Figure 3: Mean percentage of task-positive voxels in inferior parietal cortex with error bars indicating standard error of the mean. The used masks projected on a brain template and the mean percentage of task-positive voxels are presented for the inferior parietal cortex. Mask and mean percentage of active voxels are presented in black for the left hemisphere and in grey for the right hemisphere. Significant post-hoc pair-wise group comparisons are denoted by horizontal lines with asterisks ( $\mathrm{p}<.005$ ). The 16 masks used were composed of the following Automated Anatomical Labeling (AAL) regions: FRONTAL dorsolateral (dorsolateral superior and middle frontal gyri), inferior (inferior frontal gyrus opercular and triangular parts, rolandic operculum), orbital (orbital and medial orbital parts of superior frontal gyrus, orbital part of middle frontal gyrus, orbital part of inferior frontal gyrus, gyrus rectus, olfactory cortex), medial superior (medial part of superior frontal gyrus), insula (insula), motor (precentral gyrus, supplementary motor area), LIMBIC (anterior, median and posterior cingulate and paracingulate gyri), PARIETAL inferior (inferior parietal, angular and supramarginal gyri), medial (precuneus, paracentral lobule), superior (postcentral and superior parietal gyri), TEMPORAL inferior (middle and inferior temporal gyri, middle temporal pole, fusiform gyrus), medial (hippocampus, parahippocampal gyrus, amygdala), superior (superior temporal gyrus, superior temporal pole, heschl gyrus), OCCIPITAL (superior, middle and inferior ccipital gyri, cuneus, calcarine fissure, lingual gyrus), CEREBELLUM, SUBCORTICAL (caudate nucleus, putamen, pallidum, thalamus).

Developmental changes in the magnitude of activation

Next, we investigated whether older participants showed larger response amplitudes in task-related cortical areas. ROIs selected based on recent delineation of networks involved in cognitive task execution, were defined within each participant's functional data.

\section{Central Executive Network}

Within the CEN, inferior frontal junction, inferior frontal sulcus and intraparietal sulcus were selected as ROIs. One young adolescent had no positive responding 
functional cluster in the area of left inferior frontal sulcus. Therefore, 55 participants were included in the ANOVA for the CEN. The effects of both task difficulty $\left(\mathrm{F}_{(2,52)}=4.13 ; \mathrm{p}=.022\right)$ and task response $\left(\mathrm{F}_{(2,52)}=4.38 ; \mathrm{p}=.017\right)$ were modulated by age group (Figure 4). Greater PSC for endogenous compared with exogenous trials was significant in $21(\mathrm{p}=.001)$ and $17(\mathrm{p}=.017)$ year-olds, but not in 13 year-olds ( $\mathrm{p}=.397)$. Furthermore, greater activation of CEN areas during trials in which participants passed compared with trials in which they gambled was significant in $13(\mathrm{p}<.001)$ and 17 year-olds $(\mathrm{p}=.014)$, but not in 21 year-olds $(\mathrm{p}=.138)$. These findings suggest that age has a differential effect on the neurocognitive processes underlying decision making in the CEN. To the ex-

CEN IFJ

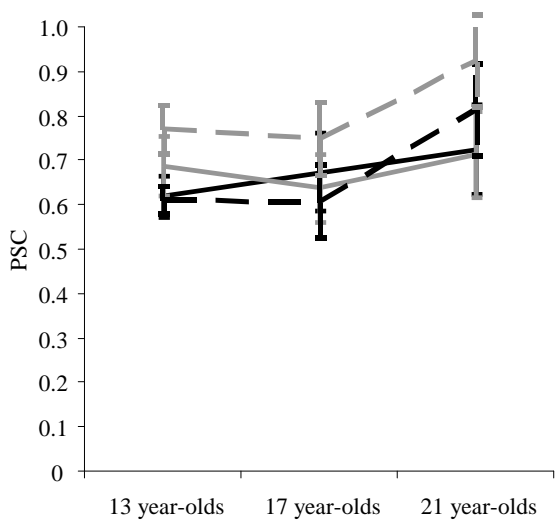

CEN IFS

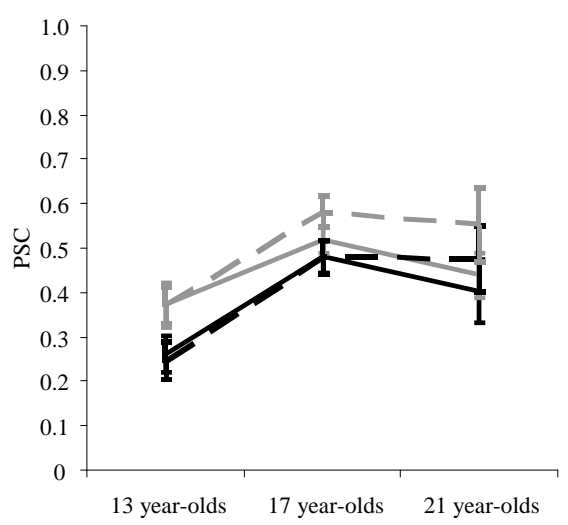

CEN IPS

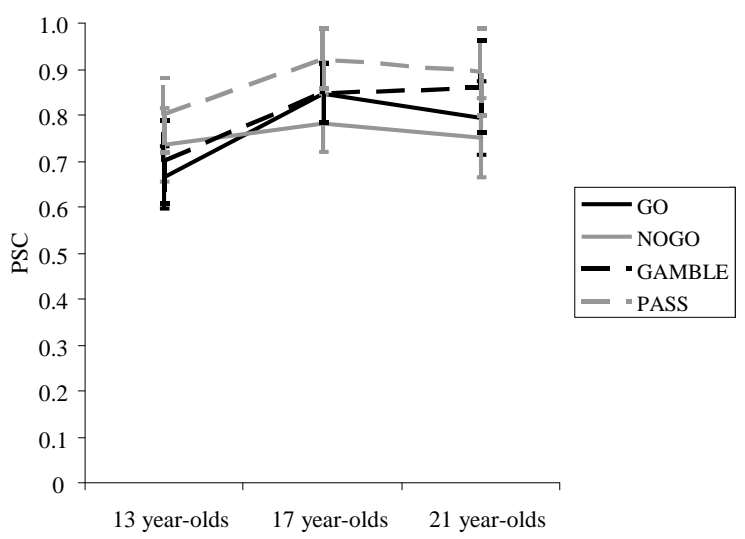


SN aIns

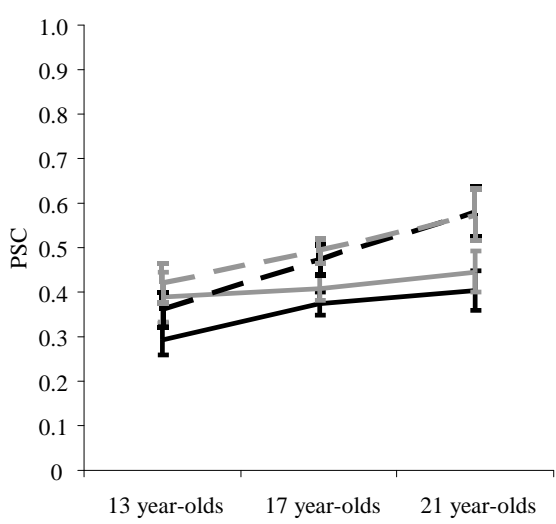

DMN IIPC

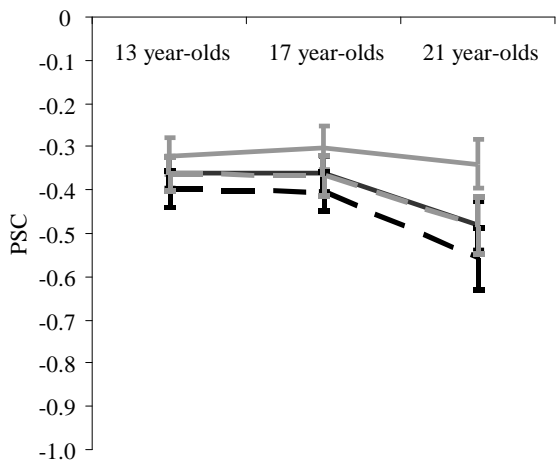

SN dACC

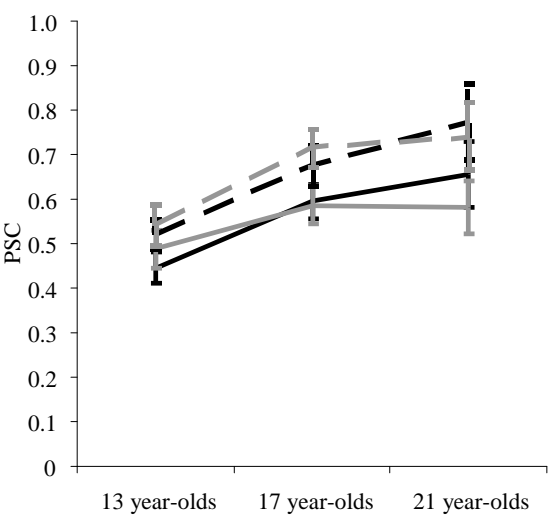

DMN PCC

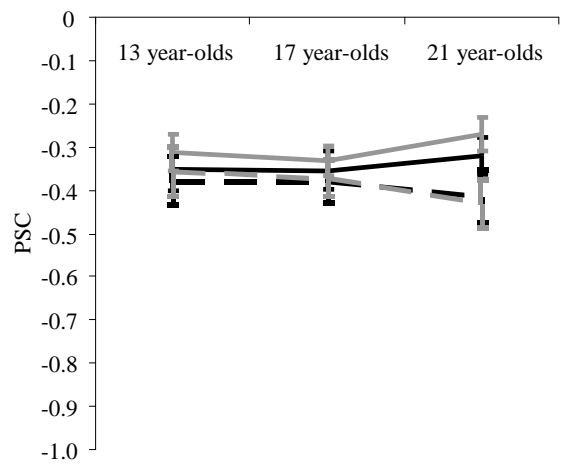

Figure 4: Age-related activation profiles of ROIs during the gambling task. Mean percent signal changes were shown for each trial type (GO, NOGO, GAMBLE, PASS) in each ROI of the Central Executive, Salience and Default Mode Networks separately. Effect of task difficulty (exogenous versus endogenous) is presented by the continuous versus dotted lines. The effect of task response (gambling versus passing) is shown by the black versus grey lines. Mean and standard deviation of the $\mathrm{x}, \mathrm{y}, \mathrm{z}$ coordinates of the ROIs across all participants were: IFJ right 42.9(4.0), 0.9(4.2), 30.0(5.6) and left -46.4(4.1), 1.1(4.4), 31.2(5.3); IFS right 40.2(5.2), 22.1(7.2), 28.8(5.5) and left -43.0(4.9), 23.4(7.1), 29.1(5.0); IPS right 27.6(5.7), 54.8(5.2), 47.4(4.3) and left -33.9(5.7), -53.5(16.1), 46.6(4.3); aIns right 30.9(3.2), 15.5(4.2), 7.3(4.3) and left -32.8(2.5), 16.7(4.1), 8.6(4.1); dACC right 3.4(2.5), 6.2(4.8), 44.8(4.4) and left -7.2(2.8), 5.6(4.4), 44.5(3.8); IIPC right 41.5(4.6), -63.8(6.1), 32.7(5.6) and left -44.4(3.9), -67.7(5.0), 32.7(5.7); PCC right 5.3(3.2), -48.4(5.1), 31.4(6.6) and left -8.9(5.2), -48.9(5.44), 31.5(8.1). PSC=percent signal change; $\mathrm{CEN}=$ central executive network; IFJ=inferior frontal junction; IFS=inferior frontal sulcus; IPS=intraparietal sulcus; $\mathrm{SN}=$ salience network; aIns=anterior insula; $\mathrm{dACC}=$ dorsal anterior cingulate cortex; $\mathrm{DMN}=$ default mode network; IIPC=lateral inferior parietal cortex; PCC=posterior cingulate cortex. 
tent that increased signal change in CEN areas reflects neurocognitive effort, the age effects observed suggest that for younger participants the endogenous relative to exogenous gambling decision was not more effortful as opposed to adults, while at the same time the decision to refrain from gambling did emerge as more effortful.

\section{Salience Network}

The dorsal part of ACC and anterior insula were selected as ROIs within the SN. There was a main effect for age group $\left(\mathrm{F}_{(2,53)}=4.37\right.$; $\left.\mathrm{p}=.017\right)$, but also an interaction effect for age group $\mathrm{x}$ task difficulty $\left(\mathrm{F}_{(2,53)}=4.26 ; \mathrm{p}=.019\right)$. In fact, the age effect was such that it increased PSC during endogenous relative to exogenous trials. This effect of decision difficulty was strongest in 21 year-olds $(\mathrm{p}<.001)$, followed by $17(\mathrm{p}<.001)$ and lowest but still significant in 13 yearolds ( $\mathrm{p}=.011)$. Likewise, age group differences in $\mathrm{SN}$ areas were significant only in endogenous trials ( $p=.005$; 13 year-olds $<21$ year-olds) but not in exogenous trials $(p=.088)$. This result suggests that the saliencing effect of a difficult decision only emerges or is increased with age during adolescence.

\section{Default Mode Network}

Functional clusters in posterior cingulate and lateral inferior parietal cortices were selected as ROIs within the DMN. One young adolescent and two old adolescents had no negative responding cluster in the left or right posterior cingulate cortex. Therefore, 53 participants were included in the ANOVA for the DMN. Greater deactivation of DMN areas during endogenous compared with exogenous trials was modulated by age group $\left(\mathrm{F}_{(2,50)}=2.54 ; \mathrm{p}=.037\right)$. This task difficulty effect was strongest in 21 year-olds $(\mathrm{p}<.001)$, followed by $13(\mathrm{p}=.029)$ and 17 year-olds $(\mathrm{p}=.055)$. So, in line with the increased signal change with age in CEN and SN areas during more challenging decisions, the 21 year-olds also show enhanced inhibition of DMN areas during more challenging trials.

\section{Correlation analyses}

In all positive responding ROIs the mean PSC was positively correlated with the task-positive activation extent in the corresponding mask (Table 3). This finding suggests that higher PSC are associated with a larger activation extent. This association is shown within each ROI, and does not suggest qualitative changes in the activation pattern. Similarly, the mean PSC in the negatively responding ROIs correlated negatively with the task-negative activation extent in corresponding masks (Table 3). These effects are not age specific, however, as they remained significant when we controlled for age.

Brain-behavior correlations were only significant in a few brain areas. Taskpositive activation extent in the occipital cortex was associated with faster reaction times $(\mathrm{r}=-.271 ; \mathrm{p}=.044)$, and in the limbic cortex with less NOGO errors 
$(\mathrm{r}=-.267 ; \mathrm{p}=.047)$. Task-negative activation extent in the parietal cortex was associated with a lower ratio of GAMBLE/ PASS trials ( $\mathrm{r}=-.284 ; \mathrm{p}=.034)$, whereas in the occipital cortex with slower reaction times ( $\mathrm{r}=.267 ; \mathrm{p}=.047)$. As above, these correlation remained (nearly) significant when we controlled for age. The mean PSC in each functional brain network was not associated with behavioral performance.

Table 3: Correlations between mean percent signal change and activation extent

\begin{tabular}{llccc}
\hline ROI & Mask & Hemisphere & $\mathrm{r}$ & $\mathrm{p}$-value \\
\hline Inferior Frontal Junction & frontal & $\mathrm{R}$ & .471 & $<.001$ \\
(IFJ) & dorsolateral & $\mathrm{L}$ & .305 & .022 \\
Inferior Frontal Sulcus & frontal & $\mathrm{R}$ & .480 & $<.001$ \\
(IFS) & dorsolateral & $\mathrm{L}$ & .358 & .007 \\
IntraParietal Sulcus & parietal & $\mathrm{R}$ & .335 & .012 \\
(IPS) & inferior & $\mathrm{L}$ & .534 & $<.001$ \\
dorsal part of Anterior Cingulate & limbic area & $\mathrm{R}$ & .474 & $<.001$ \\
Cortex (dACC) & & $\mathrm{L}$ & .630 & $<.001$ \\
anterior Insula & insula & $\mathrm{R}$ & .581 & $<.001$ \\
(aIns) & & $\mathrm{L}$ & .445 & .001 \\
Posterior Cingulate Cortex & limbic area & $\mathrm{R}$ & -.721 & $<.001$ \\
(PCC) & & $\mathrm{L}$ & -.441 & .001 \\
lateral Inferior Parietal Cortex & parietal & $\mathrm{R}$ & -.378 & .004 \\
(IIPC) & inferior & $\mathrm{L}$ & -.612 & $<.001$ \\
\hline
\end{tabular}

\section{Discussion}

The results of the present study confirmed only one aspect of the focalization hypothesis after rigorously controlling confounding variables. Functional brain activity focalized into task-specific brain areas with increasing BOLD response between ages 13 and 21. However, this was not accompanied by a change in the number of active voxels in either direction. Nor did we find any qualitative shifts in brain areas involved in executing the gambling task. In fact, the brain areas engaged in our three age groups were highly similar.

A challenging gambling decision task was used to take advantage of the later developing decision making abilities (Hooper et al., 2004; Steinberg et al., 2008b), whereas previous developmental studies often used relatively simple cognitive tasks (e.g. Durston et al., 2006; Scherf et al., 2006; Tamm et al., 2002). The behavioral results on this gambling task indicated that the age of the participants did not have an effect on their gambling behavior, neither in the speed of decision making nor in making impulsive and risky decisions. This lack of differences in gambling behavior between adolescents and adults agrees with the results of Eshel and colleagues (2007), although children aged 9 to 12 were found to make more errors compared with adults (van Leijenhorst et al., 2006). 
As is evident from these previous studies as well as our own results, absence of behavioral evidence for developmental changes in risk-taking and decision making does not preclude the possibility of finding developmental changes in the neural mechanism engaged during gambling decisions.

The first component of the focalization hypothesis states that during development task induced brain activation evolves from diffuse brain activity distributed over more brain areas than in adults, and also encompasses brain structures that are not activated by the same task in adults. Our results could not confirm this. In the whole brain analysis we did not find, even at the uncorrected significance level, any brain areas that were exclusively active in younger participants. In addition, when voxels in various parts of the brain were counted in individual data sets, we could not find any systematic decrease in activation extent with age. In fact, we found the opposite effect, increased activation extent with age in the inferior parietal cortex. Bilateral task-positive activation extent was smaller in 13 year-olds compared with 17 year-olds, and borderline significant compared with 21 year-olds in the left hemisphere. Previous studies examining agerelated changes in activation extent showed inconsistent results, with small effects (e.g. Durston et al., 2006; Galvan et al., 2006; Luna et al., 2001; Scherf et al., 2006). Earlier evidence for an age-related shift from diffuse to focal activation patterns might be mediated by confounding factors. Higher heterogeneity in younger age groups due to the use of wide age ranges and more variable anatomy and functional organization increases the chance of finding more diffuse activation patterns. In the present study these factors were controlled. Furthermore, most of previous studies examined developmental changes in activation extent over a wider age range than the present study, revealing the most robust differences between children and adults (Galvan et al., 2006; Luna et al., 2001; Scherf et al., 2006). In addition, maturational changes in functional connectivity supportive of progressive focalization were most pronounced between children and adults, with an intermediate pattern for adolescents (Kelly et al., 2009). This might suggest that a developmental shift from a diffuse to focal activation pattern might be applicable in comparing children with adults, but not within the period of adolescence.

The focalization hypothesis further states that the shift from diffuse activation towards activity confined to task-related areas is accompanied by an increase in BOLD response in these areas. A few of these emerged in the whole brain group analysis. Activation in the dorsal part of ACC and lingual gyrus was stronger in 21 year-olds compared with 13 and 17 year-olds at the FDR corrected level. In addition, exploring age-related differences at the uncorrected significance level revealed increased response amplitude with age as well in superior frontal sulcus and several occipital areas. This developmental effect was further confirmed in the analysis of percent signal change in selected regions of the major task-related brain networks. In areas of task positive net- 
works, central executive as well as salience network, the BOLD response was enhanced with increasing age and similarly, areas of the default mode network were more deactivated with age. The age-related increase in response amplitude was shown during the difficult, endogenous trials. A possible explanation for this gradual increase in BOLD strength could be the gradual maturation of the areas in these distributed functional networks during adolescence. This is in line with the observed region specific changes in cortical density, with prolonged maturation of dorsal parietal, dorsal and medial prefrontal areas during and post adolescence (Gogtay et al., 2004; Sowell et al., 1999; Sowell et al., 2001). Maturation most likely also takes the form of maturing connectivity, with more effective long range connections being installed in the course of adolescence (Fair et al., 2007, 2008; Kelly et al., 2009). Although the areas within brain networks might be available in children and adolescents, the way these areas are connected functionally is still immature and inefficient compared with adults.

The intensification of the BOLD response in task-related areas with increasing age was modulated by task conditions. The main developmental pattern that we observed in the present study was an increase in response amplitude with age during endogenous relative to exogenous decision trials, and a complementary increased deactivation of the DMN areas. This effect of task difficulty was most pronounced in 21 year-olds and to a lesser extent or not at all established in the two younger age groups. These findings agree with previous studies showing that in adults the cortical response to increased task difficulty involves the same network of brain areas working harder (Duncan \& Owen, 2000; Gould et al., 2003). Our developmental finding that this task difficulty effect matures during adolescence is confirmed by O'Hare and colleagues (2008), who did not observe it in children aged 7-10. This general developmental pattern is in CEN areas complicated by an additional interaction effect of age and task response. The response amplitude in CEN areas was enhanced during passing compared with gambling trials in 13 and 17 year-olds, but not in 21 year-olds. The CEN areas, inferior frontal junction, inferior frontal sulcus as well as intraparietal sulcus, have been associated with response inhibition (e.g. Garavan et al., 1999; Simmonds et al., 2008). This finding suggests that inhibiting risky behavior takes more effort in adolescents than young adults, which corresponds with the increased tendency to risk taking behavior during adolescence (Steinberg et al., 2008b). In 13 year-olds the CEN response was determined solely by the decision to gamble or pass, whereas in 21 year-olds the CEN response was solely determined by whether the decision was exogenous or endogenous. The 17 year-olds held an intermediate position, showing CEN response modulation by both task difficulty and task response. To the extent that response amplitude can be interpreted as a marker for the neural expenditure put into making a decision, this result indicates that for 13 year old adolescent boys it is more effortful to decide to pass, whereas for young adults it is more effortful to consider endogenously 
to come to a decision. Such changes can well be mediated by changes in other brain structures, possibly involved in socio-emotional processes that develop in the course of adolescence (Nelson et al., 2005; Steinberg, 2005, 2008a).

As a last consideration we would like to point out that developmental effects in the whole brain group analysis were very subtle and did not meet standard fMRI requirements for multiple comparison correction when examined within the averaged group data. Not until the threshold was lowered from FWE and FDR corrected to an uncorrected significance level, which is often applied in developmental studies, some more clusters were found to differ between age groups. However, using an uncorrected significance level has to be treated with great caution as the probability of false positives increases (Bennett et al., 2009; Poldrack \& Mumford, 2009). Given the subtlety of age effects relative to the variability in measured BOLD responses, developmental imaging studies should include larger sample sizes than are presently used. The weak developmental effects in brain activation were in clear contrast to the strong task induced activation patterns that were observed in all three age groups. These areas largely corresponded with previous neuroimaging studies examining decision making (e.g. Botvinick et al., 2004; Paulus, 2005; Smith et al., 2009; van Leijenhorst et al., 2006) as well as with the core functional networks involved in cognitive task execution (e.g. Dosenbach et al., 2007; Greicius et al., 2003; Sridharan et al., 2008; Stiers et al., 2010). These robust findings confirm that the gambling task was very effective in modulating the measured BOLD signal and that developmental effects were small in comparison.

In interpreting the present results, several limitations should be taken into consideration. First, our sample was restricted to male participants. Given differential trajectories of structural brain development in males and females (e.g. Giedd et al., 2006), female adolescents should be included as well in future studies to examine the influence of sex on the established developmental changes underlying decision making. Second, the age range used here covers the early/middle adolescence to young adulthood. Future studies should also include a child/early adolescent group as well as an older adult group to characterize the developmental trajectory in brain activation in a broader age range. Third, we excluded 10 adolescents in order to control for the confounding effect of head motion. This selection bias may have reduced the generalization of the present findings. Nevertheless, we think that grounds for controlling head motion weigh heaviest in examining true age effects. Namely, including young adolescents with excessive head motion would have reduced the signal to noise ratio in this group and consequently overestimated age effects.

In sum, we found only partially evidence for the hypothesized progression towards more focalized and enhanced activation in task-relevant areas between ages 13 and 21 when controlling for nuisance variables. We found no evidence for either decreased activation extent or qualitative shifts in activation patterns 
between ages 13 and 21, but revealed similar activation patterns across age groups. Developmental changes in activation magnitude evolved from no differential response in most task-related areas to an enhanced BOLD response to more difficult, endogenous task conditions with increasing age. In addition, deciding to pass as opposed to gamble exerted more effort in 13 year-olds. 


\section{References}

Achenbach, T.M., Rescorla, L.A., 2001. Manual for the ASEBA school-age forms \& profiles. Burlington, VT: University of Vermont, Research Centre for Children, Youth and Families.

Achenbach, T.M., Rescorla, L.A., 2003. Manual for ASEBA adult forms and profiles. Burlington, VT: University of Vermont, Research Centre for Children, Youth and Families.

Amodio, D.M., Frith, C.D., 2006. Meeting of minds: the medial frontal cortex and social cognition. Nature, 7, 268-277.

Ardila, A., Rosselli, M., Matute, E., Guajardo, S., 2005. The influence of the parents' educational level on the development of executive functions. Developmental Neuropsychology, 28(1), 539-560.

Bennett, C.M., Miller, M.B., Wolford, G.L., 2009. Neural correlates of interspecies perspective taking in the post-mortem Atlantic Salmon: an argument for multiple comparisons correction. In: Organization for Human Brain Mapping Abstracts.

Berl, M.M., Vaidya, C.J., Gaillard, W.D., 2006. Functional imaging of developmental and adaptive changes in neurocognition. NeuroImage, 30, 679-691.

Booth, J.R., Burman, D.D., Meyer, J.R., Lei, Z., Trommer, B.L., Davenport, N.D., et al., 2003. Neural development of selective attention and response inhibition. NeuroImage, 20, 737-751.

Botvinick, M.M., Cohen, J.D., Carter, C.S., 2004. Conflict monitoring and anterior cingulate cortex: an update. Trends in Cognitive Sciences, 8(12), 539-546.

Braver, T.S., Cohen, J.D., Nystrom, L.E., Jonides, J., Smith, E.E., Noll, D.C., 1997. A parametric study of prefrontal cortex involvement in human working memory. Neuroimage, 5, 49-62.

Brett, M., Johnsrude, I.S., Owen, A.M., 2002. The problem of functional localization in the human brain. Nature Reviews Neuroscience, 3, 243-249.

Brown, T.T., Lugar, H.M., Coalson, R.S., Miezin, F.M., Petersen, S.E., Schlaggar, B.L., 2005. Developmental changes in human cerebral functional organization for word generation. Cerebral Cortex, 15, 275-290.

Bunge, S.A., Dudukovic, N.M., Thomason, M.E., Vaidya, C.J., Gabrieli, J.D.E., 2002. Immature frontal lobe contributions to cognitive control in children: Evidence from fMRI. Neuron, 33, 301-311.

Casey, B.J., Thomas, K.M., Davidson, M.C., Kunz, K., Franzen, P.L., 2002. Dissociating striatal and hippocampal function developmentally with a stimulus-response compatibility task. The Journal of Neuroscience, 22(19), 8647-8652.

Casey, B.J., Trainor, R.J., Orendi, J.L., Schubert, A.B., Nystrom, L.E., Giedd, J.N., et al., 1997. A developmental functional MRI study of prefrontal activation during performance of a go-no-go task. Journal of Cognitive Neuroscience, 9(6), 835-847.

Choudhury, S., Blakemore, S. J., Charman, T., 2006. Social cognitive development during adolescence. Social Cognitive and Affective Neuroscience, 1(3), 165-174.

Cole, M.W., Schneider, W., 2007. The cognitive control network: Integrated cortical regions with dissociable functions. NeuroImage, 37, 343-360.

Corbetta, M., Shulman, G.L., 2002. Control of goal-directed and stimulus-driven attention in the brain. Nature Reviews Neuroscience, 3, 201-215.

Crone, E.A., Wendelkern, C., Donohue, S., van Leijenhorst, L., Bunge, S.A., 2006. Neurocognitive development of the ability to manipulate information in working memory. Proceedings of the National Academy of Sciences U.S.A., 103(24), 9315-9320. 
Dahl, R.E., 2004. Adolescent brain development: a period of vulnerabilities and opportunities. Keynote address. Annals of the New York Academy of Sciences, 1021, 1-22.

Derrfuss, J., Brass, M., Neumann, J., von Cramon, D.Y., 2005. Involvement of the inferior frontal junction in cognitive control: Meta-analyses of switching ad stroop studies. Human Brain Mapping, 25, 22-34.

Devlin, J.T., Poldrack, R.A., 2007. In praise of tedious anatomy. NeuroImage, 37, 1033-1041. Directoraat-Generaal voor de Arbeidsvoorziening (1989). Handleiding voor de functieanalyse [Function analyses manual]. Den Haag: SDU uitgeverij.

Dosenbach, N.U.F, Fair, D.A., Miezin, F.M., Cohen, A.L., Wenger, K.K., Dosenbach, R.A.T., et al., 2007. Distinct brain networks for adaptive and stable task control in humans. Proceedings of the National Academy of Sciences U.S.A., 104(26), 11073-11078.

Duncan, J., Owen, A.M., 2000. Common regions of the human frontal lobe recruited by diverse cognitive demands. Trends in Neurosciences, 23(10), 475-483.

Dunn, L.M., Dunn, L.M., 2005. Peabody Picture Vocabulary Test-III-NL. Handleiding [Manual]. Amsterdam: Harcourt Assessment B.V.

Durston, S., Casey. B.J., 2006. What have we learned about cognitive development from neuroimaging? Neuropsychologia, 44, 2149-2157.

Durston, S., Davidson, M.C., Tottenham, N., Galvan, A., Spicer, J., Fossella, J.A., et al., 2006. A shift from diffuse to focal cortical activity with development. Developmental Science, 9(1), 1-20.

Ernst, M., Nelson, E.E., Jazbec, S., McClure, E.B., Monk, C.S., Leibenluft, E., et al., 2005. Amygdala and nucleus accumbens in responses to receipt and omission of gains in adults and adolescents. NeuroImage, 25(4), 1279-1291.

Eshel, N., Nelson, E.E., Blair, J., Pine, D.S., Ernst, M., 2007. Neural substrates of choice selection in adults and adolescents: development of the ventrolateral prefrontal and anterior cingulate cortices. Neuropsychologia, 45, 1270-1279.

Fair, D.A., Cohen, A.L., Dosenbach, N.U.F., Church, J.A., Miezin, F.M., Barch, D.M., et al., 2008. The maturing architecture of the brain's default network. Proceedings of the National Academy of Sciences U.S.A., 105(10), 4028-4032.

Fair, D.A., Dosenbach, N.U.F., Church, J.A., Cohen, A.L., Brahmbhatt, S., Miezin, F.M., et al., 2007. Development of distinct control networks through segregation and integration. Proceedings of the National Academy of Sciences U.S.A., 104, 13507-13512.

Friston, K.J., Williams, S., Howard, R., Frackowiak, R.S.J., Turner, R., 1996. Movementrelated effects in fMRI time-series. Magnetic Resonance in Medicine, 35(3), 346-355.

Gaillard, W.D., Grandin, C.B., Xu, B., 2001. Developmental aspects of pediatric fMRI: considerations for image acquisition, analysis, and interpretation. NeuroImage, 13(2), 239249.

Galvan, A., Hare, T.A., Parra, C.E., Penn, J., Voss, H., Glover, G., et al., 2006. Earlier development of the accumbens relative to orbitofrontal cortex might underlie risk-taking behavior in adolescents. The Journal of Neuroscience, 26(25), 6885-6892.

Garavan, H., Ross, T.J., Stein, E.A., 1999. Right hemispheric dominance of inhibitory control: an event-related functional fMRI study. Proceedings of the National Academy of Sciences U.S.A., 96, 8301-8306.

Giedd, J.N., 2004. Structural magnetic resonance imaging of the adolescent brain. Annals of the New York Academy of Sciences, 1021, 77-85.

Giedd, J.N., Clasen, L.S., Lenroot, R., Greenstein, D., Wallace, G.L., Ordaz, S., et al., 2006. Puberty-related influences on brain development. Molecular and Cellular Endocrinology, 254-255, 154-162.

Giedd, J.N., Lalonde, F.M., Celano, M.J., White, S.L., Wallace, G.L., Lee, N.R. et al., 2009. Anatomical brain magnetic resonance imaging of typically developing children and ado- 
lescents. Journal of the American Academy of Child and Adolescent Psychiatry, 48(5), 465-470.

Giorgio, A., Watkins, K.E., Chadwick, M., James, S., Winmill, L., Douaud, G., et al., 2010. Longitudinal changes in grey and white matter during adolescence. NeuroImage, 49, 94103.

Gogtay, N., Giedd, J.N., Lusk, L., Hayashi, K.M., Greenstein, D., Vaituzis, A.C., et al., 2004. Dynamic mapping of human cortical development during childhood through early adulthood. Proceedings of the National Academy of Sciences U.S.A., 101(21), 81748179.

Gould, R.L., Brown, R.G., Owen, A.M., ffytche, D.H., Howard, R.J., 2003. fMRI BOLD response to increasing task difficulty during successful paired associates learning. NeuroImage, 20, 1006-1019.

Greicius, M.D., Krasnow, B., Reiss, A.L., Menon, V., 2003. Functional connectivity in the resting brain: A network analysis of the default mode hypothesis. Proceedings of the National Academy of Sciences U.S.A., 100(1), 253-258.

Hooper, C.J., Luciana, M., Conklin, H.M., Yarger, R.S., 2004. Adolescents' performance on the Iowa Gambling Task: implications for the development of decision making and ventromedial prefrontal cortex. Developmental Psychology, 40(6), 1148-1158.

Huizinga, M., Dolan, C. V., van der Molen, M.W., 2006. Age-related changes in executive functions: Developmental trends and a latent variable analysis. Neuropsychologia, 44, 2017-2036.

Johnstone, T., Ores Walsh, K.S., Greischar, L.L., Alexander, A.L., Fox, A.S., Davidson, R.., et al., 2006. Motion correction and the use of motion covariates in multiple-subject fMRI analysis. Human Brain Mapping, 27, 779-788.

Kelly, A.M.C., Di Martino, A., Uddin, L.Q., Shehzad, Z., Gee1, D.G., Reiss, P.T., et al., 2009. Development of anterior cingulate functional connectivity from late childhood to early adulthood. Cerebral Cortex, 19, 640-657.

Keulers, E.H.H., Evers, E.A.T., Stiers, P., Jolles, J., 2010. Age, sex and pubertal phase influence mentalizing about emotions and actions in adolescents. Developmental Neuropsychology, 35(4), 555-569.

Klenberg, L., Korkman, M., Lahti-Nuuttila, P., 2001. Differential development of attention and executive functions in 3- to 12-year-old Finnish children. Developmental Neuropsychology, 20(1), 407-428.

Luciana, M., Conklin, H.M., Hooper, C.J., Yarger, R.S., 2005. The development of nonverbal working memory and executive control processes in adolescents. Child Development, 76(3), 697-712.

Luna, B., Garver, K.E., Urban, T.A., Lazar, N.A., Sweeney, J.A., 2004. Maturation of cognitive processes from late childhood to adulthood. Child Development, 75(5), 1357-1372.

Luna, B., Thulborn, K.R., Munoz, D.P., Merriam, E.P., Garver, K.E., Minshew, N.J., et al., 2001. Maturation of widely distributed brain function subserves cognitive development. NeuroImage, 13, 786-793.

Maldjian, J.A., Laurienti, P.J., Burdette, J.B., Kraft, R.A., 2003. An automated method for neuroanatomic and cytoarchitectonic atlas-based interrogation of fMRI data sets. NeuroImage, 19, 1233-1239.

Manoach, D.S., 2003. Prefrontal cortex dysfunction during working memory performance in schizophrenia: reconciling discrepant findings. Schizophrenia Research, 60, 285-298.

Manoach, D.S., Gollub, R.L., Benson, E.S., Searl, M.M., Goff, D.C., Halpern, E., et al., 2000. Schizophrenic subjects show aberrant fMRI activation of dorsolateral prefrontal cortex and basal ganglia during working memory performance. Biological Psychiatry, 48, 99109. 
May, R., Hunter, M., Gabriel, R., 1993. NPfact 1.0. Victoria, Canada: University of Victoria, Department of Psychology.

Mayer, A.R., Franco, A.R., Ling, J., Canive, J.M., 2007. Assessment and quantification of head motion in neuropsychiatric functional imaging research as applied to schizophrenia. Journal of the International Neuropsychological Society, 13, 839-845.

Mennes, M., Stiers, P., Lagae, L., Van den Bergh, B., 2006. Long-term cognitive sequelae of antenatal maternal anxiety: involvement of the orbitofrontal cortex. Neuroscience and Biobehavioral Reviews, 30, 1078-1086.

Mennes, M., Van den Bergh, B., Lagae, L., Stiers, P., 2009. Developmental brain alterations in 17 year old boys are related to antenatal maternal anxiety. Clinical Neurophysiology, 120, 1116-1122.

Mennes, M., Wouters, H., van den Bergh, B., Lagae, L., Stiers, P., 2008. ERP correlates of complex human decision making in a gambling paradigm: Detection and resolution of conflict. Psychophysiology, 45, 714-720.

Nelson, E.E., Leibenluft, E., McClure, E.B., Pine, D.S., 2005. The social re-orientation of adolescence: A neuroscience perspective on the process and relation to psychopathology. Psychological Medicine, 35, 163-174.

O’Hare, E.D., Lu, L.H., Houston, S.M., Bookheimer, S.Y., Sowell, E.R., 2008. Neurodevelopmental changes in verbal working memory load-dependency: An fMRI investigation. NeuroImage, 42, 1678-1685.

Paulus, M.P., 2005. Neurobiology of decision-making: Quo vadis? Cognitive Brain Research, 23, 2-10.

Paus, T., 2005. Mapping brain maturation and cognitive development during adolescence. Trends in Cognitive Sciences, 9, 60-68.

Paus, T., Keshavan, M., Giedd, J.N., 2008. Why do many psychiatric disorders emerge during adolescence? Nature Reviews Neuroscience, 9, 947-957.

Poldrack, R.A., Mumford, J.A., 2009. Independence in ROI analysis: where is the voodoo? Social Cognitive and Affective Neuroscience, 4, 208-213.

Raven, J., Raven, J.C., Court, J.H., 1998. Manual for Raven’s Progressive Matrices and Vocabulary Scales. Section 1: General Overview. Harcourt Assessment, San Antonio TX.

Rubia, K., Smith, A.B., Taylor, E., Brammer, M., 2007. Linear age-correlated functional development of right inferior fronto-striato-cerebellar networks during response inhibition and anterior cingulate during error-related processes. Human Brain Mapping, 28, 1163-1177.

Rubia, K., Smith, A.B., Woolley, J., Nosarti, C., Heyman, I., Taylor, E., et al., 2006. Progressive increase of frontostriatal brain activation from childhood to adulthood during event-related tasks of cognitive control. Human Brain Mapping, 27, 973-993.

Scherf, K.S., Sweeney, J.A., Luna, B., 2006. Brain basis of developmental change in visuospatial working memory. Journal of Cognitive Neuroscience, 18(7), 1045-1058.

Seeley, W.W., Menon, V., Schatzberg, A.F., Keller, J., Glover, G.H., Kenna, H., et al., 2007. Dissociable intrinsic connectivity networks for salience processing and executive control. The Journal of Neuroscience, 27(9), 2349-2356.

Simmonds, D.J., Pekar, J.J., Mostofsky, S.H., 2008. Meta-analysis of go/no-go tasks demonstrating that fMRI activation associated with response inhibition is task- dependent. Neuropsychologia, 46, 224-232.

Slifer, K.J., Cataldo, M.F., Cataldo, M.D., Llorente, A.M., Gerson, A.C., 1993. Behavior analysis of motion control for pediatric neuro-imaging. Journal of Applied Behavior Analysis, 269, 469-470. 
Smith, B.W., Mitchell, D.G.V., Hardin, M.G., Jazbec, S., Fridberg, D., Blair, R.J.R., et al., 2009. Neural substrates of reward magnitude, probability, and risk during a wheel of fortune decision-making task. NeuroImage, 44, 600-609.

Sowell, E.R., Thompson, P.M., Holmes, C.J., Jernigan, T.L., Toga, A.W., 1999. In vivo evidence for post-adolescent brain maturation in frontal and striatal regions. Nature neuroscience, 2(10), 859-861.

Sowell, E.R., Thompson, P.M., Tessner, K.D., Toga, A.W., 2001. Mapping continued brain growth and gray matter density reduction in dorsal frontal cortex: Inverse relationships during postadolescent brain maturation. The Journal of Neuroscience, 21(22), 88198829.

Spear, L.P., 2000. The adolescent brain and age-related behavioral manifestations. Neuroscience and Biobehavioral Reviews, 24, 417-463.

Sridharan, D., Levitin, D.J., Menon, V., 2008. A critical role for the right fronto-insular cortex in switching between central-executive and default-mode networks. Proceedings of the National Academy of Sciences U.S.A., 105(34), 12569-12574.

Steinberg, L., 2005. Cognitive and affective development in adolescence. Trends in Cognitive Sciences, 9(2), 69-74.

Steinberg, L., 2008a. A social neuroscience perspective on adolescent risk-taking. Developmental Review, 28, 78-106.

Steinberg, L., Albert, D., Cauffman, E., Banich, M., Graham, S., Woolard, J., 2008b. Age differences in sensation seeking and impulsivity as indexed by behavior and self report: Evidence for a dual systems model. Developmental Psychology, 44(6), 1764-1778.

Stiers, P., Mennes, M., Sunaert, S., 2010. Distributed task coding throughout the multiple demand network of the human frontal-insular cortex. NeuroImage, 52(1), 252-262.

Talairach, J., Tournoux, P., 1988. Co-planar stereotaxic atlas of the human brain. Thieme Medical Publishers Inc, New York.

Tamm, L., Menon, V., Reiss, A.L., 2002. Maturation of brain function associated with response inhibition. Journal of the American Academy of Child \& Adolescence Psychiatry, 41(10), 1231-123.

Thomas, K.M., King, S.W., Franzen, P.L., Welsh, T.F., Berkowitz, A.L., Noll, D.C. et al., 1999. A developmental functional MRI study of spatial working memory. NeuroImage, 10, 327-338.

Thomason, M.E., Chang, C.E., Glover, G.H., Gabrieli, J.D.E., Greicius, M.D., Gotliba, I.H., 2008. Default-mode function and task-induced deactivation have overlapping brain substrates in children. NeuroImage, 41, 1493-1503.

Toga, A.W., Thompson, P.M., 2007. What is where and why is important. NeuroImage, 37, 1045-1049.

Tzourio-Mazoyer, N., Landeau, B., Papathanassiou, D., Crivello, F., Etard, O., Delcroix, N., et al., 2002. Automated anatomical labeling of activations in SPM using a macroscopic anatomical parcellation of the MNI single-subject brain. NeuroImage, 15(1), 273-289.

Uylings, H.B.M., Rajkowsk, G., Sanz-Arigita, E., Amunts, K., Zilles, K., 2005. Consequences of large interindividual variability for human brain atlases: converging macroscopical imaging and microscopical neuroanatomy. Anatomy \& Embryology, 210, 423-431.

Van Leijenhorst, L., Crone, E.A., Bunge, S.A., 2006. Neural correlates of developmental differences in risk estimation and feedback processing. Neuropsychologia, 44, 21582170.

Velanova, K., Wheeler, M.E., Luna, B., 2008. Maturational changes in anterior cingulate and frontoparietal recruitment support the development of error processing and inhibitory control. Cerebral Cortex, 18, 2505-2522. 
Wenger, K.K., Visscher, K.M., Miezin, F.M., Petersen, S.E., Schlaggar, B.L., 2004. Comparison of sustained and transient activity in children and adults using a mixed blocked/event-related fMRI design. NeuroImage, 22, 975-985.

Wilke, M., Holland, S.K., 2003. Variability of gray and white matter during normal development: a voxel-based MRI analysis. NeuroReport, 14(15), 1887-1890.

Yoo, S., Choi, B., Juh, R., Pae, C., Lee, C., 2005. Head motion analysis during cognitive fMRI examination: Application in patients with schizophrenia. Neuroscience Research, 53, 84-90. 



\section{Chapter}

\section{Decoding age from task-induced brain activation in adolescence}

Esther H.H. Keulers, Alexandros Goulas, Jelle Jolles and Peter Stiers

Manuscript. 


\begin{abstract}
In the present study we examined whether age-related changes in brain activation are widespread throughout the brain, and whether these changes are independent of the particular cognitive function performed. This view on functional maturation is based on widespread maturational changes in anatomy and functional connectivity, and contrasts with previous developmental fMRI studies that focussed on changes in brain responsiveness associated with particular changes in behavior. Next to the traditional, univariate voxel-wise age group comparisons, we applied multivariate pattern classification analysis to study developmental changes in brain activation during a simple gonogo task in male adolescents. This multivariate approach was highly accurate in discriminating between 13 year-olds and respectively 17 and 21 year-olds. The multivariate results suggest that developmental changes are spatially distributed throughout the brain, affecting the responsiveness of a wide range of task positive and default mode regions. Moreover, these distributed age-distinctive patterns in brain activation generalize to different conditions of the gonogo task, and even to a totally different gambling task. This suggests that functional brain maturation in adolescence is independent of the specific cognitive processes executed. In contrast to the accurate classification of adolescent age groups by the multivariate analysis, univariate voxel-wise analysis revealed few age-related clusters, that only differed between 13 and 21 year-olds. Thus, multivariate pattern classification analysis might be more suited for studying subtle and widespread developmental changes in brain activation than an univariate approach.
\end{abstract}




\section{Introduction}

Adolescence is a developmental period between child and adulthood of profound changes in body and behavior. Because adolescents are prone to risk taking behavior, leading to rising numbers of accidents and early death, as well as an increased incidence of behavioral disorders (Casey et al., 2008; Dahl, 2004; Steinberg, 2008a), these changes in behavior have often been subject of research. In recent years this research also included many functional neuroimaging studies, assuming that these changes in behavior will lead to the markers of functional brain maturation that are characteristic for adolescence. For example, studies on the late maturation of response control have asked what changes in functional activation during response control tasks may be responsible for this improved behavioral performance (e.g. Rubia et al., 2006; Tamm et al., 2002). Similarly, more recent attempts searched for the neural correlates of adolescent changes in social cognition (e.g. Blakemore et al., 2007) and risky decision making (e.g. Steinberg et al., 2008b; van Leijenhorst et al., 2010).

In contrast to this approach, most studies of anatomical changes underlying adolescent brain maturation do not focus on typical aspects of adolescent behavior, but instead look for changes throughout the brain. These structural MRI studies typically find widespread changes in both subcortical and cortical structures, involving mostly association cortices in the major lobes, as well as in white matter tracts between them (Giedd, 2004; Giedd et al., 2009; Giorgio et al., 2010; Gogtay et al., 2004). In addition, increase in long-range (i.e. between spatially remote areas) functional connectivity has been demonstrated in widespread brain networks with age, such as anterior cingulate-insular connections in the task positive network and posterior cingulate-ventral medial prefrontal connections in the default mode network (Fair et al., 2007, 2008; Kelly et al., 2009).

In the present study we ask whether also at the functional level adolescent brain maturation is characterized by widespread changes, rather than changes in brain responsiveness associated with the maturation of specific cognitive functions. This alternative view on functional maturation follows the shift in the conception of the functional organization of the cortex, from cortical specialization associated with a particular cognitive function towards widespread functional networks that are involved in a wide range of cognitive functions. Metaanalyses of neuroimaging studies into specific cognitive functions have shown that the same brain areas are active during performance of many cognitive tasks, regardless of the specific task demands (Derrfuss et al., 2004; Duncan \& Owen, 2000; Mennes et al., 2006; Stiers et al., 2010). Further evidence supports the idea that these areas work together as networks, both during task execution and 
when the brain is at rest (Cole \& Schneider, 2007; Dosenbach et al., 2007; Sridharan et al., 2008).

To address this question of widespread functional maturation we start from the ability to inhibit a prepotent response. Later on we will investigate how specific these maturational changes are to this particular cognitive function. This simple aspect of response control is already acquired by the end of preschool years, but performance improvements continue well into early adolescence (Huizinga et al., 2006; Luna et al., 2004). Successful inhibition in adults is associated with increased activity in a predominantly right lateralized network of lateral and medial prefrontal, parietal and subcortical regions (Buchsbaum et al., 2005; Garavan et al., 2006; Simmonds et al., 2008). The right ventrolateral prefrontal cortex in particular has been implicated (Aron \& Poldrack, 2005; Garavan et al., 2006).

Functional MRI studies examining developmental changes in brain activation associated with response inhibition reported inconsistent results. Some fMRI studies have shown an age-related increase in frontal activation during inhibition, viz in anterior cingulate cortex and inferior frontal gyrus. These results suggest that areas specifically involved in response inhibition, are stronger activated with increasing age (e.g. Bunge et al., 2002; Rubia et al., 2006, 2007; Tamm et al., 2002). However, other fMRI studies demonstrated a decrease in activation in frontal areas with age, notably in lateral and medial frontal areas. This finding of an initially more diffuse pattern of activation in frontal, taskrelevant areas has been explained as reflecting more cognitive effort in children compared with adults during successful inhibition (e.g. Booth et al., 2003; Casey et al., 1997; Durston et al., 2002). Moreover, the reported age-related effects are subtle, and do not survive statistical significance thresholds that take into account the normally recommended corrections for multiple comparisons (Bennett et al., 2009; Poldrack \& Mumford, 2009).

The inconsistency in results may partially reflect the intrinsic shortcoming of the traditional voxel-wise group comparisons to determine subtle and distributed changes in brain activation. Univariate voxel-wise analysis requires sufficient signal difference at a single voxel to obtain statistical significance, while the developmental changes may comprise of small changes in responsiveness distributed over a large number of voxels. That would make multivariate pattern classification analysis a more suited approach for developmental studies, because it takes many voxels simultaneously into account in the search for consistent spatial activation patterns that are maximally discriminant between age groups. Given the age-related changes in the configuration of functional brain networks (e.g. Fair et al., 2007), it makes sense that studying development in terms of changing activation patterns is more sensitive than studying changes in individual voxels (Bray et al., 2009). In addition to increased sensitivity (De Martino et al., 2008), multivariate pattern classification analysis may also pro- 
vide better consistency. In developmental fMRI studies, statistical differences revealed by univariate voxel-wise age group comparisons may reflect true differences as well as chance differences in the noise of the data. This might be particularly true when the significance thresholds have to be lowered to reveal the weak age effects. Consequently, reported age group differences might reflect sample specific patterns that do not generalize towards other samples, yielding inconsistency across studies. Multivariate pattern classification, on the other hand, directly tests generalizability of the age-distinctive pattern learned by the classifier to a new data sample that is independent of the training sample. The degree of generalizability to the test sample determines the relevance of the found pattern.

The feature of generalizability also allows us in the present study to address in a direct way the question whether age differentiating patterns are specific to particular cognitive functions or not. Different task conditions intend to control the requirement of specific cognitive processes at particular times during the task. By using data from one task condition for training and another task condition for testing, it can be directly investigated whether the pattern learned is specific to the cognitive demands imposed by the different task conditions. However, the most rigorous test for the independency of functional maturation patters from specific cognitive functions comprises of training the classifier on functional data from one particular task and testing it on data from the same group of participants performing a completely different task.

In the study described here we administered an event related gonogo task to three male adolescent age groups of respectively 13, 17 and 21 years of age. Participants had to press a response key whenever a letter was presented, but had to withhold their response when the nogo stimulus was presented (letter "A"). The present task included go oddball next to go and nogo trials. These oddball trials required a response comparable to the go trials, but occurred as often as the nogo trials to control for the effect of low-frequent occurrence. Next to the traditional univariate voxel-wise analysis, we applied multivariate pattern classification analysis to establish whether age-related functional changes were widespread throughout the brain as indicated by maturational changes in anatomy and functional connectivity. In addition, we examined whether developmental changes in brain activation are independent from the particular cognitive task performed, by investigating whether classification generalizes between the three different conditions of the gonogo task. Moreover, we used the fMRI data obtained from the same sample of participants while they performed a challenging gambling task (Keulers et al., 2010) and investigated whether the classifier trained on the data from the gonogo task was successful in classifying participants according to age from their functional activation data during the gambling task. 


\section{Materials and methods}

\section{Participants and procedure}

A total of 70 participants from the seventh (12/13 year-olds) and eleventh (16/17 year-olds) grades of pre-university education as well as university students (20/21 year-olds) were included. This sample was also described in a previous study, which examined developmental differences during a challenging gambling task (Keulers et al., 2010). Participants were recruited through advertisements and information talks. All participants had normal or corrected-to-normal vision, were free from psychiatric or neurological abnormalities, never repeated or skipped a grade, did not use medication that could influence cognitive functioning and were screened for MRI contra-indications. Written informed consent was obtained from all participants and their parents in case participants aged 17 or less. Participants received travel expenses and a fee of 25 euros. The study was approved by the ethical committee of the Faculty Psychology and Neuroscience of the Maastricht University. Data from 1 participant were excluded due to a motivational problem, 2 participants were excluded due to technical problems, 3 participants due to poor behavioral performance (error rate $>30 \%$ ) and 7 participants due to excessive head motion (see section Functional Magnetic Resonance Imaging data - Preprocessing for details). This resulted in a final sample of 57 male, right-handed participants divided into three age groups as follows: 13 year-olds $(\mathrm{N}=19$, mean age $=12.9, \mathrm{SD}=0.3$, range $12.3-13.7), 17$ year-olds $(\mathrm{N}=21$, mean age $=17.0, \mathrm{SD}=0.4$, range $16.3-17.7)$ and 21 year-olds $(\mathrm{N}=17$, mean age $=21.0, \mathrm{SD}=0.6$, range 20.1-21.9).

Participants attended a training session on average one week before the scan session. The training session served to familiarize participants with the scanning environment and procedure, and to practice the experimental tasks using a mock scanner. In addition, the youngest age group received a training in the mock scanner to reduce motion (Slifer et al., 1993). Participants viewed a cartoon videotape in the mock scanner for 2 x 10 minutes, which was halted whenever the participants moved their head. Furthermore, two neuropsychological tests were administered to obtain estimates of intelligence. Age groups did neither differ on an estimate of verbal intelligence $\left(\mathrm{M}=113.89, \mathrm{SD}=8.23, \mathrm{~F}_{(2,54)}=2.38\right.$; $\mathrm{p}=.102$ ) as assessed with the Peabody Picture Vocabulary Test-III-NL (Dunn \& Dunn, 2005) nor on an estimate of nonverbal intelligence $(\mathrm{M}=121.96, \mathrm{SD}=5.17$, $\left.\mathrm{F}_{(2,54)}=1.40 ; \mathrm{p}=.256\right)$ as assessed with the Raven Standard Progressive Matrices (Raven et al., 1998). The Child Behavior Checklist and the Youth Self Report were administered in the two youngest age groups (Achenbach \& Rescorla, 2001) whereas the Adult Self Report (Achenbach \& Rescorla, 2003) was presented in the student group to screen for behavioral problems. All participants had scores on the total problem scale of these questionnaires within 1 SD of the 
mean of a normative standardized sample. Furthermore, age groups did not differ on the total problem scale of the self report versions $\left(F_{(2,52}=1.46 ; p=.242\right)$. In addition, level of parental education was inquired with a commonly-used Dutch education scale (Directoraat-Generaal voor Arbeidsvoorziening, 1989), as higher levels of parental education have been associated with higher performance on executive functioning tasks (Ardila et al., 2005; Klenberg et al., 2001). Fifteen parent-pairs (26.3\%) had a moderate level of education (elementary school to general secondary education) and 42 (73.7\%) parent-pairs had a high level of education (high vocational education to doctoral degree). Level of parental education did not differ between age groups $\left(\chi_{(2)}^{2}=2.66 ; p=.264\right)$.

Task

The gonogo task consisted of three letter stimuli (“ $\mathrm{X}$ ”, "B" and “ $A$ ”) that were randomly presented using a fast event-related design. Participants were instructed to press the response key every time a letter appeared on the screen, but to withhold their response when an " $\mathrm{A}$ " appeared. The letter stimuli corresponded to three conditions: GO ("X”), GOoddball ("B”) and NOGO (“A”). In $60 \%$ of all trials, the letter " $\mathrm{X}$ " was shown, whereas the letters " $\mathrm{A}$ " and " $\mathrm{B}$ " both appeared in $20 \%$ of all trials. The higher percentage of GO stimuli allowed for the build up of a prepotent tendency to respond. The GOoddball stimuli required a response comparable to the GO trials, but occurred as often as the NOGO trials to control for the effect of low-frequent occurrence (Chikazoe et al., 2009; Hampshire et al., 2010). Letters were presented visually at the centre of the screen, with a duration of 200 ms. During inter stimulus intervals a fixation cross was shown and jittering was applied to these intervals. The inter stimulus interval varied between $1000 \mathrm{~ms}$ and $2000 \mathrm{~ms}$, with an average duration of 1500 ms. In addition, null-events with the same duration and inter stimulus interval as the letter trials were included (33\% of all trials) in which the fixation cross remained visible, to allow for better separation of the BOLD responses. The task was presented in two runs, each consisting of 260 trials, of which 175 were letters. Duration of each run was 7.48 minutes. The mean and variability of reaction times, errors of omission (misses) and errors of commission (false alarms) were recorded. The task was programmed using E-Prime (version 1.1; Psychology Software Tools, 2002).

\section{Behavioral analyses}

The statistical package SPSS 16.0 was used for the analyses. Developmental changes in speed (i.e. mean reaction time), intra-individual speed variability (i.e. standard deviation of reaction time/mean reaction time (Stuss et al., 2003)) and accuracy (i.e. percentage of false alarms and misses) on the gonogo task were examined. Regarding the mean and variability of reaction times, two analyses of 
variance (ANOVAs) were conducted with age group (3 levels) as betweensubject variable and trial type (2 levels: GO vs GOoddball) as within-subject variable. The reaction time data did not violate the parametric assumption of ANOVA. The number of false alarms (i.e. failure to withhold response on NOGO trials) and misses (i.e. failure to react on GO and GOoddball trials) were analysed with a non-parametric Kruskal-Walis test due to non-normality. If statistically significant effects were found, post hoc comparisons were performed.

Functional Magnetic Resonance Imaging data

Image acquisition

A Siemens MAGNETOM Allegra 3T MRI head-only scanner was employed. Head motion was constrained by the use of foam padding. A total number of 32 axial slices covering the whole brain including the cerebellum were imaged by using a T2*-weighted gradient echo planner pulse sequence $(\mathrm{TR}=2000 \mathrm{~ms}$, TE $=30 \mathrm{~ms}, \mathrm{FA}=90, \mathrm{FOV}=224$, slice thickness $=4 \mathrm{~mm}$, matrix size $=64 \times 64$, flip angle $=90^{\circ}$ ). Voxel size was $3.5 \times 3.5 \times 4 \mathrm{~mm}$. A T1-weighted anatomical scan was acquired to aid with spatial normalization ( $\mathrm{TR}=2250 \mathrm{msec}$, $\mathrm{TE}=2.6$ msec, flip angle $=9^{\circ}, \mathrm{FOV}=256 \mathrm{~mm}$, slice thickness $=1 \mathrm{~mm}$, matrix size $=256$ $\mathrm{x} 256$, number of slices $=192$ ). Voxel size was 1 x 1 x $1 \mathrm{~mm}$. Slice scanning order was ascending interleaved.

\section{Preprocessing}

Data were preprocessed using BrainVoyager QX, version 1.9 (Brain Innovation, Maastricht, The Netherlands). Images were corrected for slice scan time differences, followed by rigid body motion correction, high-pass temporal filtering, and spatial data smoothing using a Gaussian kernel with a $4 \mathrm{~mm}$ full width at half maximum. Functional data were coregistered with the anatomical volume using rigid body transformation and manual adjustments to obtain optimal fit by visual inspection, if necessary. Subsequently, both functional and structural volumes were transformed into standard stereotaxic space using Talairach normalization.

The degree of head motion during scanning was greater in the 13 year-olds compared with the two older age groups on the two most affected parameters (Mayer et al., 2007; Yoo et al., 2005), viz translation in the $\mathrm{z}$ direction (Tz; $\mathrm{F}_{(2}$, $61)=5.89 ; \mathrm{p}=.005)$ and rotation in the $\mathrm{x}$ direction $\left(\mathrm{Rx} ; \mathrm{F}_{(2,61)}=6.62 ; \mathrm{p}=.002\right)$. In order to control for the confounding effect of head motion we applied 3 corrections to our data. Firstly, head motion within one scan may distort the measured fMRI signal by spatial misallocation. Therefore, we identified scans during which head motion exceeded a particular threshold, viz absolute motion difference between 2 successive scans in z-direction greater than $0.4 \mathrm{~mm}\left(1 / 10^{\text {th }}\right.$ of the 
voxel size), and rotation in the $\mathrm{x}$ dimension greater than 0.26 degrees (angle corresponding to $0.4 \mathrm{~mm}$ z-displacement of frontopolar voxels, assuming the rotation point in middle of brain is $88 \mathrm{~mm}$ from the anterior end of the brain frontal pole (Talairach \& Tournoux, 1988)). Task trials (taking into account the hemodynamic response function delay) cause signal intensity changes with peak BOLD values 5 to 6 seconds after their occurrence. These signal changes may overlap in time with head motion a few scans later. Consequently, these signal changes are contaminated with within-scan head motion. Therefore, trials in a preceding time window of 1 to 8 seconds of the identified scan were modeled as an additional event of no interest. Because this procedure reduces the number of trials available in the events of interest, we set an upper limit of $25 \%$ to the number of trials discarded in this way. As a consequence, 1 young adolescents and 1 old adolescent were excluded because they exceeded this upper limit. After applying this procedure, age groups still differed on the motion parameters for the remaining scans, viz $\mathrm{Tz}\left(\mathrm{F}_{(2,59)}=6.94 ; \mathrm{p}=.003\right)$ and $\mathrm{Rx}\left(\mathrm{F}_{(2,59)}=5.70\right.$; $\mathrm{p}=.005)$. Therefore, as a second measure to equate groups on the motion parameters it was unavoidable to exclude an additional 5 young adolescents with the largest head motion on the $\mathrm{Tz}$ and $\mathrm{Rx}$ parameters. This was effective in eliminating head motion differences between the 3 age groups, viz $\mathrm{Tz}\left(\mathrm{F}_{(2}\right.$, $\left.{ }_{54}=2.53 ; \mathrm{p}=.089\right)$ and $\mathrm{Rx}\left(\mathrm{F}_{(2,54)}=2.87 ; \mathrm{p}=.065\right)$. Thirdly, head motion between successive scans causes signal intensity changes due to inhomogeneities in the magnetic field which are unrelated to the task (Friston et al., 1996). Therefore, we modeled this source of signal variance by including the 6 motion parameters as covariates of no interest in our general linear model (Friston et al., 1996; Johnstone et al., 2006).

\section{Imaging analyses}

Statistical analyses were performed on individual participants data by using the general linear model in SPM5 (Welcome Department of Cognitive Neurology, London, UK). The fMRI time series were modeled as series of events convolved with a canonical hemodynamic response function. A design matrix was set up to model all task conditions of interest (GO, GOoddball and NOGO trials) and the 6 motion parameters. Error trials and trials contaminated with head motion were modeled as two separate predictors that were ignored in the further analyses.

Individual percent signal change maps were created for each condition of interest, based on the estimated weights for each of the conditions, and used as data for a second level analysis in which subject was treated as a random effect. A whole brain, voxel-wise general linear model (GLM) with task condition (3 levels) as within-subject variable and age group (3 levels) as between-subject variable was performed. First, we identified brain areas underlying the execution of the gonogo task in a contrast that pooled over task conditions and age groups, at the Family Wise Error corrected level (FWE<.05). 
Second, we examined age group differences using the univariate voxel-wise GLM. In order to make the GLM results directly comparable with the multivariate pattern (MVP) classification results, we examined developmental effects for each task condition and each pair-wise age group combination separately (i.e. GO trials 13 vs. 17 year-olds; GO trials 13 vs. 21 year-olds; GO trials 17 vs. 21 year-olds; GOoddball trials 13 vs. 17 year-olds; GOoddball trials 13 vs. 21 yearolds; GOoddball trials 17 vs. 21 year-olds; NOGO trials 13 vs. 17 year-olds; NOGO trials 13 vs. 21 year-olds; NOGO trials 17 vs. 21 year-olds). We reported results of these analyses using the False Discovery Rate (FDR $<.05)$ error corrected level, which is a less stringent whole brain correction for multiple comparisons (Genovese et al., 2002).

Third, we applied MVP classification analysis to the percent signal change maps from the first level analysis in order to examine differences between pairwise age groups, for each task condition. A whole brain approach was applied, in which all grey matter voxels were examined simultaneously. Grey matter voxels were selected across participants within each pair-wise group comparison, first by creating an individual spatially smoothed grey matter mask (Gaussian kernel with $9 \mathrm{~mm}$ full width at half maximum), and second by finding the common voxels across these individual masks that had a grey matter density of at least 0.2 in all masks. The voxels included in the analysis are referred to as features. For each participant, the values of the included voxels in the percent signal change map were converted to a vector/exemplar and labelled according to the age group a participant belonged to. For the MVP classification analysis a pattern recognition algorithm was adopted, the Recursive Feature Elimination (RFE) (De Martino et al., 2008; Guyon et al., 2002). This strategy belongs to the so-called wrapper methods, in which the classifier itself is used to select features that appear to be relevant for the classification at hand. For the implementation of the algorithm a version of Support Vector Machines called Least Squares Support Vector Machines (Suykens et al., 2002) and a linear kernel were adopted (http://www.esat.kuleuven.be/sista/lssvmlab/). First, for each task condition and each pair-wise age group combination the dataset was split into $\mathrm{N}$ folds ( $\mathrm{N}=10$ for comparisons between 13 and 17 year-olds, $\mathrm{N}=9$ for 13 versus 21 year-olds, and $\mathrm{N}=19$ for 17 versus 21 year-olds). A cross-validation approach was adopted leaving out data from one fold, functioning as a test set. The remaining data were used as training samples and were further divided in S overlapping splits ( $\mathrm{S}=3$ for comparisons between 13 and 17 year-olds, $\mathrm{S}=4$ for 13 versus 21 year-olds, and $S=3$ for 17 versus 21 year-olds). The classifier was trained separately in each of these splits in order to establish a mathematical function that best discriminates between the age groups. Absolute weights were calculated for each feature/voxel, which coded for the importance of each voxel to the classification. Subsequently, the average absolute weights across the splits was computed. Classification accuracy for this iteration was assessed on the 
independent test set, not used in the training. Iteratively, the process of training and testing the classifier was repeated 15 times, i.e. 15 iterations. The $30 \%$ of the initial features/voxels with the smallest weights were discarded at each iteration, after which the remaining features/voxels were used as training samples in the next iteration. To make the classification result independent of the choice of training and test set, we repeated these steps $\mathrm{N}$ times, each time leaving out data from a different fold as a test set. We generalized the classification performance by averaging accuracies over the $\mathrm{N}$ folds for each of the 15 iterations. To arrive at a final generalization performance we selected the iteration with the highest classification accuracy. The $\mathrm{N}$ weight vectors obtained at this best iteration were mapped to the 3D space and averaged as well. In this way a discriminative map was obtained with voxels that appear most discriminative at the majority of the $\mathrm{N}$ folds, having the highest weights. To evaluate the significance of the acquired accuracy values from the described cross-validation, we performed randomisation statistics with 1000 permutations and cross-validation (Mourão-Miranda et al., 2005). In each permutation, the analysis was performed as before but now the age group class labels were randomly assigned to the vectors. Accuracies from these permutations (at the iteration with the highest classification accuracy in the original analysis) that exceeded or were equal to the accuracy obtained from the original analysis were counted. Dividing this number by 1000 , a $\mathrm{p}$ value was obtained.

Fourth, to examine whether functional brain maturation is specific to a particular cognitive function or not, we first explored the generalizability of the age discriminating patterns to the different conditions of the gonogo task. For each pair-wise age group combination, we used percent signal change maps from two conditions of the gonogo task as training set, while the maps from the third gonogo condition was used as test set. This yielded three folds for crossvalidation, one for each gonogo condition. Second, we established the accuracy with which the age discriminative pattern found by training the classifier on the gonogo data, allowed to classify the same subjects while performing a very different cognitive task, i.e. a gambling task (Keulers et al., 2010). In this gambling task participants had to choose between gambling for one of two options or for passing, in order to cumulate as many points as possible (Mennes et al., 2008; Stiers et al., 2010). Four trial groups along two dimensions were defined: 1) exogenous versus endogenous trials depending on whether stimulus characteristics suggested the appropriate choice or not, and 2) gambling versus passing trials depending on whether a button was pressed or not (Mennes et al, 2008). For each pair-wise age group combination, the percent signal change maps from all three gonogo conditions were used as training set. In addition, the percent signal changes maps from the four different task conditions within the gambling task were used as test set. There were small differences in participants included in either the gonogo (training) or gambling (testing) data. Of the current partici- 
pants included in the gonogo data, three 13 year-olds, two 17 year-olds and two 21 year-olds were not included in the gambling data. In addition, two 13 yearolds, two 17 year-olds and two 21 year-olds were included in the gambling but not in the gonogo data.

Since the MVP classification analysis looks for discriminating voxel response patterns between age groups, this approach gives no directional information of age-related changes in brain activation. Therefore, we performed regions of interest (ROI) based age group analyses to examine the direction as well as the specific pattern of developmental changes between ages 13 and 21 in BOLD response. A selection of regions that contributed to age group classification were picked out. The final ROIs were identified based on the local maximum of a cluster combined with a radius of $6 \mathrm{~mm}$, based on the MVP results for the pooled gonogo conditions between 13 and 21 year-olds. For each ROI, the average Percent Signal Change (PSC) was extracted for each of the 3 gonogo conditions. Only the $20 \%$ voxels with the highest PSC were included to make the amplitude assessments maximally independent of the extent of activation. For each ROI, the PSC was compared between age groups by conducting an ANOVA with task condition (3 levels) as within-subject variable and age group (3 levels) as between-subject variable. The assumption of equal error variances across groups (Levene's test) was met for all analyses. Alpha was set at .05 for each ANOVA to give maximal sensitivity. If statistically significant effects were found, post hoc comparisons were performed.

\section{Results}

\section{Behavioral performance}

The behavioral results showed an overall decrease in mean reaction time with age $\left(\mathrm{F}_{(2,54)}=13.13 ; \mathrm{p}<.001\right)$. The 13 year-olds reacted slower compared with 17 $(\mathrm{p}=.022)$ and $21(<.001)$ year-olds, and 17 year-olds in their part were slower than 21 year-olds $(\mathrm{p}=.043)$. In addition, the ANOVA showed a main effect of trial type with longer reaction times on GOoddball compared with $\mathrm{GO}$ trials $\left(\mathrm{F}_{(2}\right.$, 54) $=14.25 ; \mathrm{p}<.001$ ). This suggests that reacting on less frequent GOoddball trials is more difficult compared with frequent GO trials. There was no significant interaction between age group and trial type.

Regarding the speed variability, the ANOVA revealed a main effect for age group $\left(\mathrm{F}_{(2,54)}=15.15 ; \mathrm{p}<.001\right)$ and an age group $\mathrm{x}$ trial type interaction effect $\left(\mathrm{F}_{(2}\right.$, 54) $=3.67 ; \mathrm{p}=.032$ ). On GO trials the 13 year-olds showed more variability in reaction times than $17(\mathrm{p}<.001)$ and $21(\mathrm{p}<.001)$ year-olds, whereas on GOoddball trials both 13 and 17 year-olds showed more speed variability compared with 21 year-olds (respectively $\mathrm{p}<.001$ and $\mathrm{p}=.004$ ). 
The number of misses on GO $\left(\chi_{(2)}^{2}=15.74 ; p<.001\right)$ and GOoddball $\left(\chi_{(2)}^{2}=6.99\right.$; $\mathrm{p}=.030)$ trials decreased with age. The 13 year-olds failed more often to respond on GO trials than the $17(\mathrm{p}<.001)$ and $21(\mathrm{p}<.001)$ year-olds and on GOoddball trials compared with 21 year-olds $(\mathrm{p}=.017)$. The number of false alarms did not differ between age groups $\left(\chi_{(2)}^{2}=3.67 ; p=.160\right)$.

As an age-effect in erroneous responses on NOGO trials was lacking, these results do not support the idea of maturation in the ability to inhibit a prepotent response during adolescence. Task performance in general, however, does become more efficient as evidenced by decreased mean reaction time, reaction time variability and number of misses.

\section{Brain activation during the gonogo task}

Positive responding areas during performance of the gonogo task were found in the anterior cingulate cortex, anterior insula, inferior frontal sulcus, subcortical and occipital regions, which have been described as part of task positive networks (e.g. Dosenbach et al., 2007; Sridharan et al., 2008; Stiers et al., 2010). In addition, the posterior cingulate cortex, superior temporal and parahippocampal gyri among others, which have been described as part of the default mode network, were deactivated during task execution (e.g. Dosenbach et al., 2007; Greicius et al., 2003; Stiers et al., 2010). These findings confirm that the used gonogo task was effective in modulating the BOLD signal in distributed functional brain networks. An overview of all significant activation clusters during gonogo performance are reported in Supplementary Table 1. The identified task positive and negative responding areas were subsequently used as a reference frame to interpret developmental changes in brain activation.

\section{Developmental effects revealed by the univariate voxel-wise analysis}

Differences in brain activation between age groups during the execution of the gonogo task as revealed by the GLM analysis are described in Table 1 . These univariate voxel-wise comparisons did not find a lot of differences in brain activation between age groups. The majority of significant clusters was revealed between 13 and 21 year-olds, for all three task conditions. Conversely, there were almost no clusters that significantly differed between 13 and 17 year-olds on the one hand, and between 17 and 21 year-olds on the other hand. The majority of age-affected clusters fall within or partially overlap (i.e. located at the border) with the earlier identified task positive and task negative responding areas. In addition, a few age-related clusters did not overlap with task related areas (Table 1). To further explore developmental effects in the univariate GLM, we evaluated the voxel-wise age group comparisons at the uncorrected level as well $(\mathrm{p}<.001 ; \mathrm{F}=11.23$; Figure 1). This yielded for each task condition and pairwise age group combination only a few, small additional clusters. 
Table 1: Results of the general linear model pair-wise age group comparisons for each gonogo condition compared to rest

\begin{tabular}{|c|c|c|c|c|c|c|c|}
\hline \multirow{2}{*}{$\begin{array}{l}\text { Task } \\
\text { condition }\end{array}$} & \multirow{2}{*}{$\begin{array}{l}\text { Age group } \\
\text { comparison }\end{array}$} & \multirow{2}{*}{ Brain area } & TAL & \multirow[t]{2}{*}{ Size } & \multirow[t]{2}{*}{ F-value } & \multirow{2}{*}{$\begin{array}{l}\mathrm{P}_{\mathrm{FDR}} \\
\text { corrected }\end{array}$} & \multirow{2}{*}{$\begin{array}{l}\text { Task } \\
\text { pos/ } \\
\text { neg\# }\end{array}$} \\
\hline & & & $\mathrm{x} \quad \mathrm{y}$ & & & & \\
\hline \multirow[t]{13}{*}{$\overline{\mathrm{GO}}$} & 13 vs 17 & l precentral sulcus & $-36-2451$ & 6 & 23.96 & .042 & pos \\
\hline & \multirow[t]{11}{*}{13 vs 21} & r STG & $57-3315$ & 28 & 33.04 & .001 & \\
\hline & & m SMA & $0-1557$ & 49 & 28.95 & .003 & pos \\
\hline & & r cerebellum culmen & $21-63-24$ & 34 & 26.70 & .003 & pos \\
\hline & & 1 inf. precentral sulcus & $-51-933$ & 26 & 19.69 & .018 & pos \\
\hline & & $\mathrm{r}$ thalamus VMP & $9-183$ & 10 & 18.98 & .020 & \\
\hline & & r postcentral sulcus & $27-3651$ & 7 & 17.39 & .027 & neg \\
\hline & & l parahip. gyrus & $-24-48-3$ & 6 & 16.68 & .033 & neg \\
\hline & & l postcentral sulcus & $-57-2436$ & 8 & 16.26 & .036 & pos \\
\hline & & l cerebellum culmen & $-36-54-24$ & 9 & 16.02 & .038 & pos \\
\hline & & l trans. temp. gyrus & $-45-3012$ & 8 & 15.77 & .040 & neg \\
\hline & & $\mathrm{m}$ posterior cingulate & $-6-4536$ & 10 & 15.24 & .046 & neg \\
\hline & 17 vs 21 & r cerebellum culmen & $21-63-24$ & 26 & 26.99 & .017 & pos \\
\hline GO & 13 vs 17 & no significant clusters & & & & & \\
\hline \multirow[t]{3}{*}{ oddball } & 13 vs 21 & r lingual gyrus & \multirow{3}{*}{$\begin{array}{l}18-510 \\
24-66-24\end{array}$} & 78 & 23.13 & .048 & neg \\
\hline & & r cerebellum & & 12 & 21.64 & .048 & pos \\
\hline & 17 vs 21 & no significant clusters & & & & & \\
\hline \multirow[t]{9}{*}{ NOGO } & 13 vs 17 & no significant clusters & & & & & \\
\hline & 13 vs 21 & r IPS & $27-6633$ & 43 & 25.33 & .011 & pos \\
\hline & & I SFS & -243633 & 8 & 24.20 & .011 & neg \\
\hline & & 1 inf. precentral sulcus & $-51-639$ & 9 & 24.15 & .011 & pos \\
\hline & & m ventral PFC & 3453 & 42 & 23.99 & .011 & neg \\
\hline & & $\mathrm{r}$ inf. precentral sulcus & $39-630$ & 16 & 23.27 & .013 & pos \\
\hline & & I STG & $-60-3918$ & 6 & 18.78 & .031 & \\
\hline & & r cuneus & \multirow[t]{2}{*}{$27-7215$} & 7 & 17.47 & .046 & \\
\hline & 17 vs 21 & no significant clusters & & & & & \\
\hline
\end{tabular}

Note. \# Indication whether activation clusters fall within and/ or overlap with task positive responding areas (=pos), task negative responding areas (=neg), or do not overlap with taskrelated areas at all (empty). Size in number of voxels. $\mathrm{TAL}=$ Talairach coordinates; FDR= False Discovery Rate error corrected level; $\mathrm{r}=$ right hemisphere; l= left hemisphere; $\mathrm{m}=$ medial; STG= Superior Temporal Gyrus; SMA= Supplementary Motor Area; inf.= inferior; $\mathrm{VMP}=$ ventral medial nucleus of the thalamus; parahip.= parahippocampal; trans.= transverse; temp.=temporal; IPS= Intraparietal Sulcus; SFS= Superior Frontal Sulcus; PFC= Prefrontal Cortex.

These results confirm that developmental changes in brain activation are subtle and often fail to reach significance in univariate GLM, even when lowering the statistical threshold. This finding corresponds to the subtlety of reported age effects in other developmental fMRI studies. 


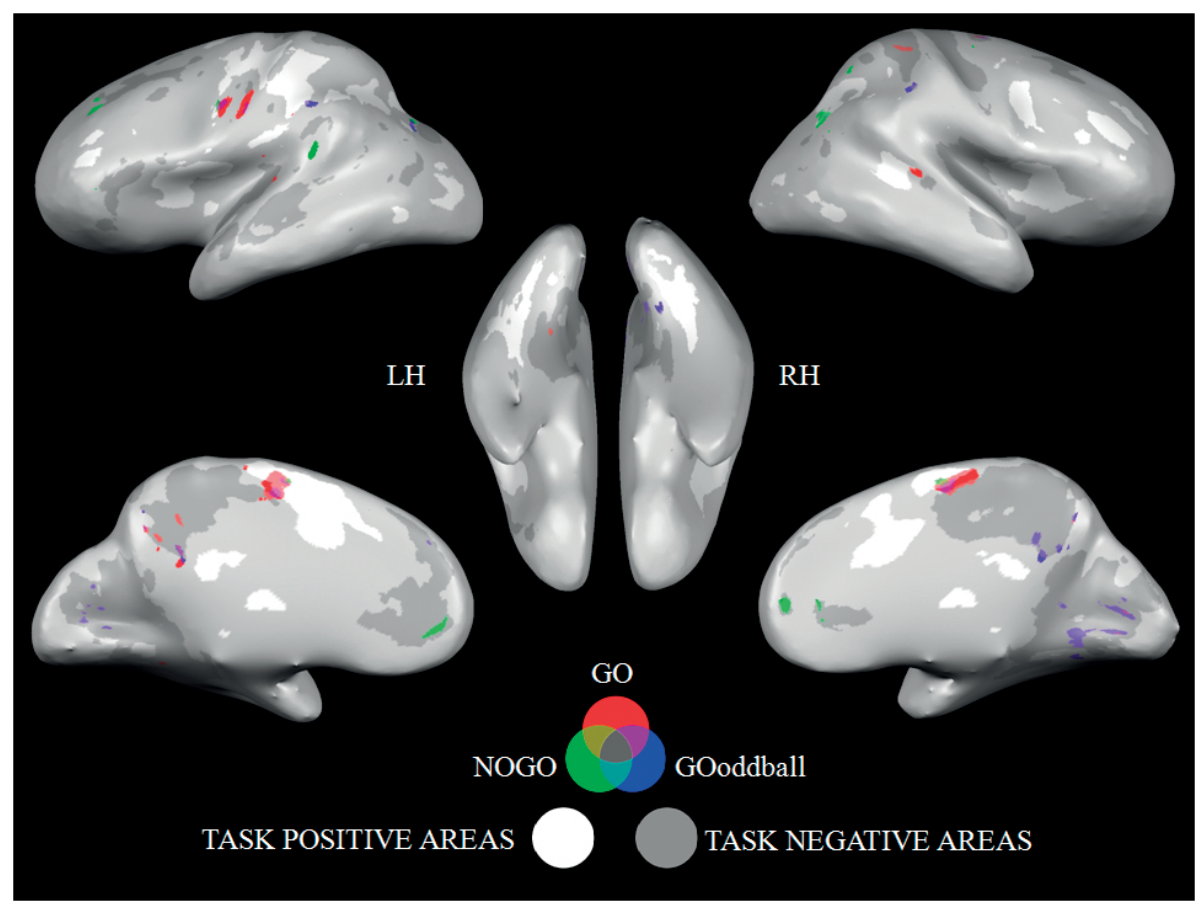

Figure 1: Differences in brain activation between 13 and 21 year-olds during gonogo performance revealed by the univariate voxel-wise analysis. Task positive (white, $\mathrm{T}=2.44$ ) and task negative (grey, $\mathrm{T}=2.23$ ) responding areas independent of task condition and age group are visualized at the False Discovery Rate (FDR) error corrected level. These identified task positive and negative areas are used as a reference frame to interpret the location of developmental findings. Differences in brain activation between 13 and 21 year-olds revealed by the univariate general linear model are presented at the uncorrected level of $\mathrm{p}=.001(\mathrm{~F}=11.23)$. Age group differences are presented separately for each task condition: GO (red), GOoddball (blue) and NOGO (green) trials.

Developmental effects revealed by multivariate pattern classification analysis

The MVP classification analysis was applied to the percent signal change maps from the first level analysis in order to examine differences between pair-wise age groups, for each task condition.

\section{Classification performance}

The multivariate pattern classification analysis proved to be an effective tool in discriminating between adolescent age groups. Classification accuracies per iteration for each task condition and pair-wise age group combination, are shown in Figure 2. The highest accuracies were obtained in discriminating 13 from 21 year-olds, for all task conditions (GO: 80.8\%; GOoddball: 80.1\%; NOGO: $84.0 \%$ ). Of 1000 random permutations only $0.1-0.3 \%$ resulted in higher accuracy values than the observed ones. 
A 13 versus 17 year-olds

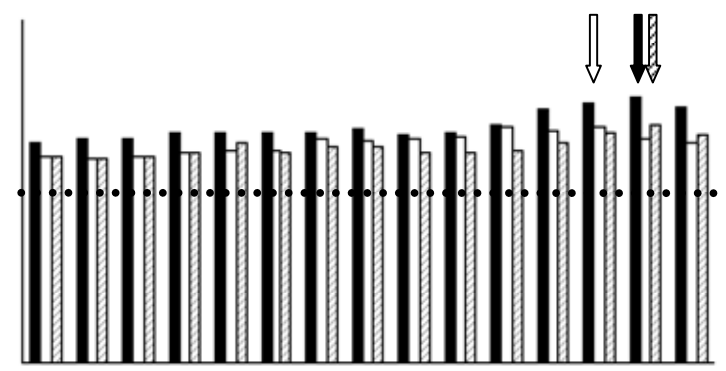

B 13 versus 21 year-olds

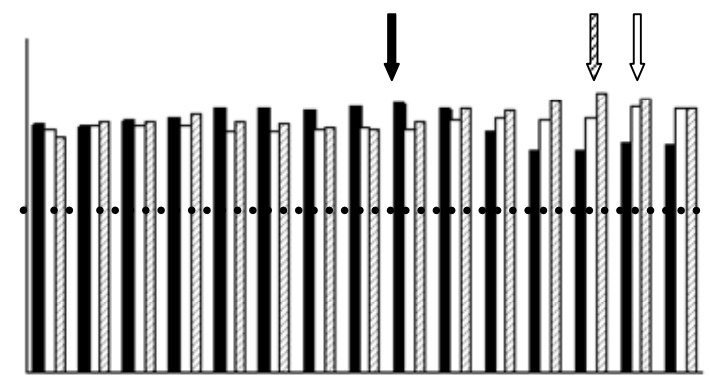

C 17 versus 21 year-olds



- GO $\square$ GOoddball $\square$ NOGO

Figure 2: Mean accuracy per iteration obtained by multivariate pattern classification analysis for each task condition and pair-wise age group combination. Each graph shows for each iteration the mean accuracy over folds for discriminating between respectively 13 and 17 year-olds (A), 13 and 21 year-olds (B), and 17 and 21 year-olds (C). The three columns represent the task conditions: GO (black), GOoddball (white) and NOGO (striped) trials. The dotted line represents chance level. The arrows indicate the iteration with the highest accuracy over folds, for each task condition. The mean discriminative map corresponding with this iteration was selected, one for each gonogo condition. 
The actual classification results can therefore be considered statistically highly significant (GO: $\mathrm{p}=.001$; GOoddball: $\mathrm{p}=.003$; NOGO: $\mathrm{p}=.001$ ). Categorizing 13 against 17 year-olds was correct in $77.5 \%(p=.004)$ based on signal changes during GO trials, in 68.9\% ( $\mathrm{p}=.031)$ during GOoddball trials and in $70.0 \%$ $(\mathrm{p}=.020)$ during NOGO trials. Lower and non-significant accuracies were observed in discriminating between 17 and 21 year-olds (GO: 62.3\%, p>.01; GOoddball: 58.8\%, $\mathrm{p}>.01$; NOGO: 50.9\%, $\mathrm{p}>.01$ ), suggesting that functional maturation mostly takes place between ages 13 and 17 .

\section{Pattern of brain areas contributing to age group classification}

The discriminative weight maps showing the spatial pattern by which age groups significantly differ for each task condition are shown in Figure 3A (13 versus 17 year-olds) and Figure 3B (13 versus 21 year-olds). Voxel clusters collectively contributing to age group classification were distributed throughout the whole brain, rather than being confined to one or more individual regions. First, some of these brain regions are associated with the default mode network, as they fall within or partially overlap with task-induced deactivated areas (Figure 3). These are evident in ventral medial prefrontal cortex, posterior cingulate cortex and anterior calcarine sulcus. In addition, discriminating regions were found in bilateral temporal and parahippocampal gyri, areas that also have been included in the default mode network (Beckmann et al., 2005; Dosenbach et al., 2007; Greicius et al., 2003). Second, several contributing regions were found that fall within or partially overlap with activated areas during execution of the gonogo task (Figure 3). For both go conditions, discriminating clusters emerged in supplementary motor area, precentral and postcentral sulci, and posterior insula, all of which are included in the sensory-motor network as described in resting state imaging studies (Beckmann et al., 2005; Damoiseaux et al. 2006; De Luca et al., 2006). In addition, voxels in attention related areas of the right intraparietal sulcus and frontal eye field (Corbetta \& Shulman, 2002; Fox et al., 2006) contributed to age discrimination. In the visual processing network (Beckmann et al., 2005; Damoiseaux et al. 2006; De Luca et al., 2006), areas in bilateral fusiform and lingual gyri contributed to age group classification. Furthermore, voxels clusters in areas of the prefrontal cortex associated with salience and central executive networks (Dosenbach et al., 2007; Sridharan et al., 2008) were found to contribute to age group discrimination. These included the anterior cingulate cortex and anterior insula, although the latter only contributed in the 13 versus 17 year-olds comparison. In addition, a cluster was found in the left inferior frontal junction, although other areas of the central executive network did not contribute to age group classification. Third, some of the regions contributing to age group discrimination did not overlap with positive or negative task responding areas, namely in the precuneus and left inferior frontal sulcus. 
A 13 versus 17 year-olds

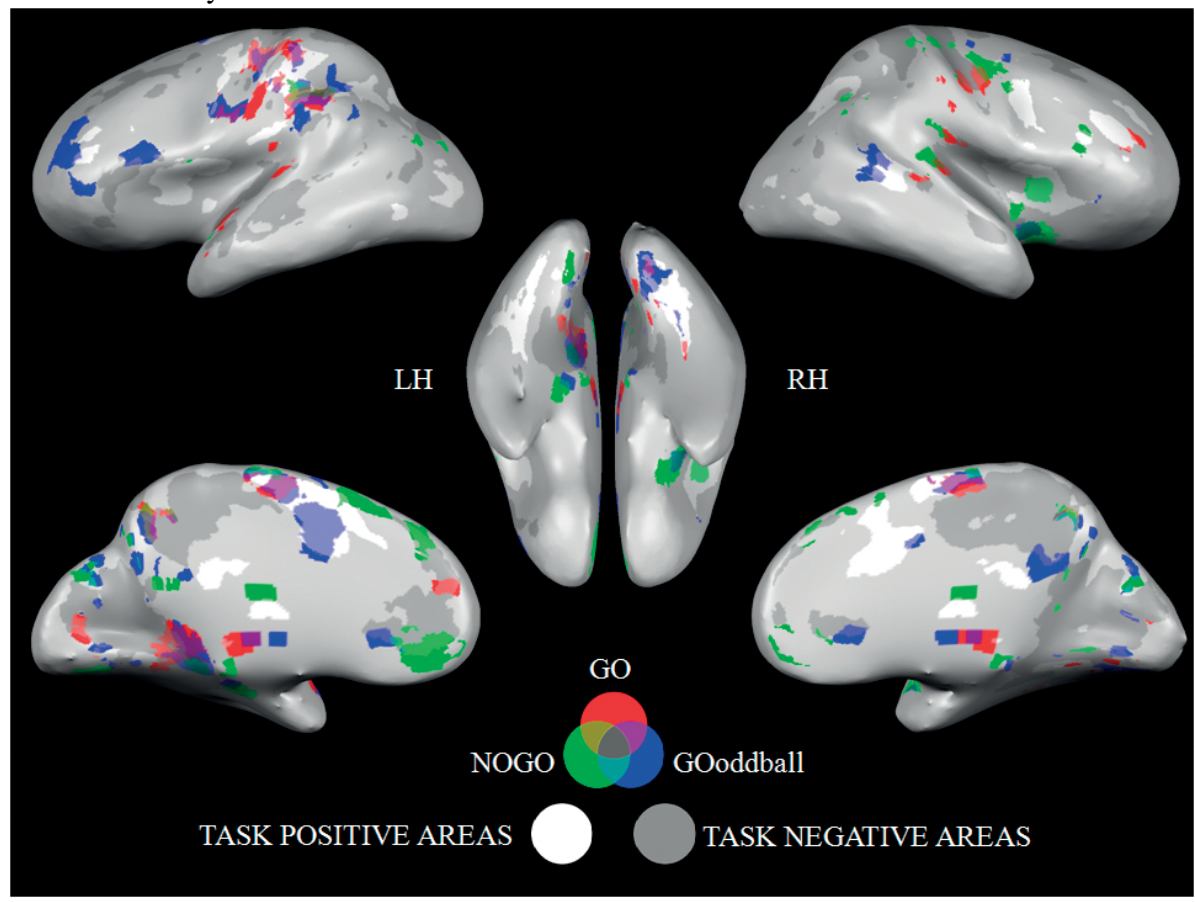

B 13 versus 21 year-olds

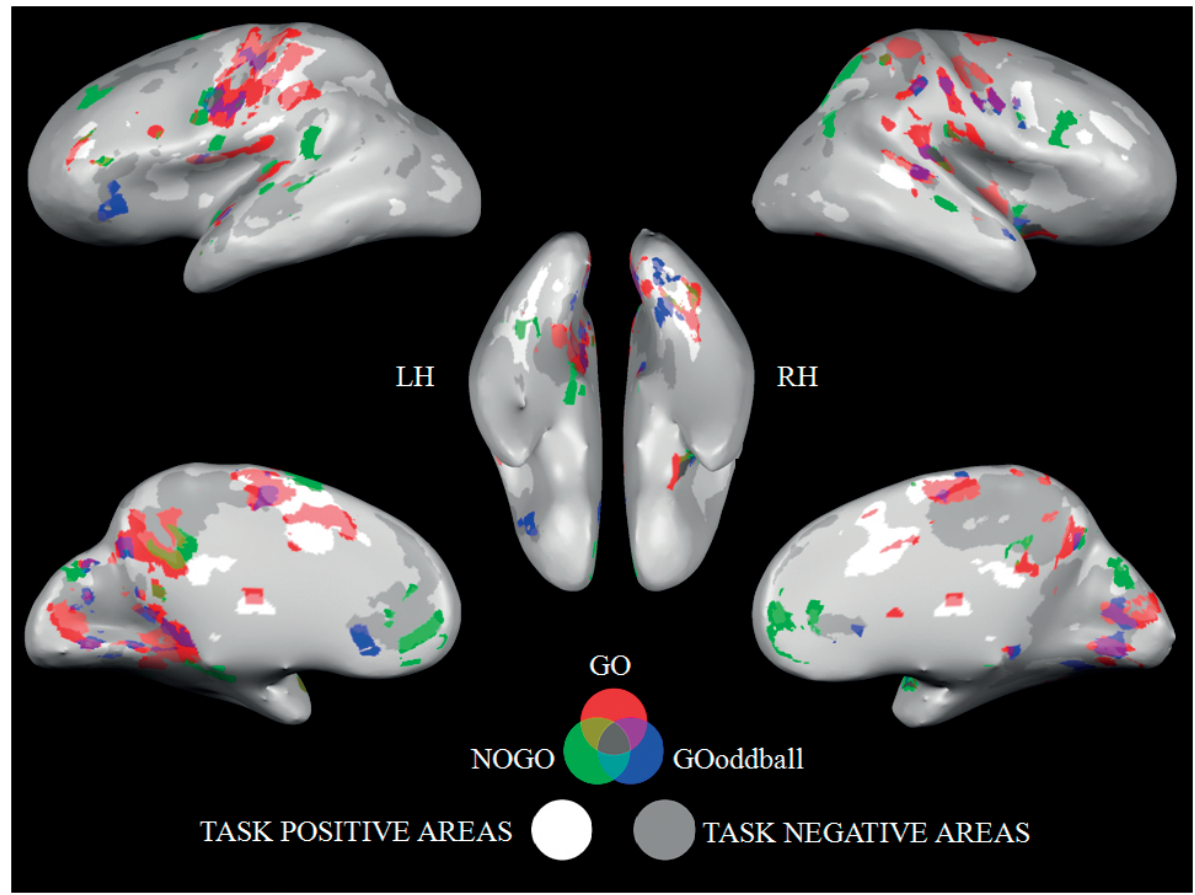


Figure 3: Pattern of brain areas contributing to age group discrimination revealed by multivariate pattern classification analyses. Task positive (white, $\mathrm{T}=2.44$ ) and task negative (grey, $\mathrm{T}=2.23$ ) responding areas independent of task condition and age group are visualized at the False Discovery Rate (FDR) error corrected level. These identified task positive and negative areas are used as a reference frame to interpret the location of developmental findings. The $30 \%$ most discriminating voxels of the weight maps corresponding to the best iteration are visualized here. Weight maps are presented for, respectively, 13 versus 17 year-olds (A) and 13 versus 21 year-olds (B), as well as for each task condition separately: GO (red), GOoddball (blue) and NOGO (green) trials.

These results show that both task positive and task negative brain networks as well as brain regions not specifically related to the gonogo task, collectively differentiate between activation patterns of 13 versus respectively 17 and 21 year-olds.

\section{Elaboration of feature selection parameters}

Because parameters in the MVP classification procedure have an effect on the results obtained, we performed the analysis with different settings for initial and subsequent iterative feature selection. In the results above, we used multivariate feature selection by discarding the $30 \%$ least contributing voxels at each iteration (total number of iterations $=15$ ), whereas there was no initial voxel selection. It has been shown, however, that initial exclusion of functionally nonresponsive voxels (univariate GLM analysis) can significantly improve the performance of the recursive feature elimination procedure (e.g. De Martino et al., 2008; Ryali et al., 2010). To this end, we repeated the classification analysis for the initial non-significant age group comparison of 17 versus 21 year-olds with the following settings: 50 iterations and discarding the $10 \%$ least contributing voxels on each iteration. Moreover, we performed an initial voxel selection based on univariate responsiveness (De Martino et al., 2008). Since the optimal number of voxels for initial reduction is not known in advance, we established the optimal number empirically. We ran a cross-validation within the training set of each fold. This consists of repeating the same classification with a different number of voxels ranging from the 4000 to the 8000 univariate most responsive voxels, in steps of 2000 voxels. The voxel number yielding the highest accuracy is selected for each task condition, and subsequently the algorithm was run with this optimal amount of initial voxels. Initial voxel selection boosted accuracies to a statistical significant level for the 17 versus 21 year-olds classification, with the highest accuracies reached in the last iteration steps, i.e. step 49 or 50 (GO: 70.2\%, $\mathrm{p}=.023$; GOoddball: 72.8\%, $\mathrm{p}=.019$; NOGO: 67.5\%, $\mathrm{p}=.055$ ). These results suggest that the functional activation differences between 17 and 21 yearolds concern small areas of relatively few voxels, which the classification was not able to pick up in the whole brain approach with fewer iterations. 
Generalizability of age-distinctive functional patterns

Although the voxel maps in Figure 3 show differences between conditions of the gonogo task in voxels contributing to classification, it does not necessarily follow that the discrimination patterns are specific to the different cognitive demands in each of the conditions. The patterns in Figure 3 also reflect the artificial decision to visualize only the $30 \%$ voxels with the highest weights. To directly test the specificity of the distributed age discriminating patterns for the different conditions of the gonogo task, the classifier was trained on percent signal change maps from two gonogo conditions and tested on the maps from the other gonogo condition, yielding three folds for cross-validation. Classification accuracies for all pair-wise age group combinations proved to be very high: 96.6\% for 13 versus 17 year-olds, $95.7 \%$ for 13 versus 21 year-olds, and $97.9 \%$ for 17 versus 21 year-olds. These results suggest that the age-distinctive pattern of functional activation is not specific to particular conditions of the gonogo task.

To investigate the generalizability of the distributed discriminating age patterns over cognitive functions even further, we established the accuracy with which the discriminative activation pattern found by training the classifier on the current gonogo data, allowed to classify the same participants while performing a very different cognitive task. Most of the current participants also participated in our previous study with a challenging and complex gambling task (Keulers et al., 2010). In this gambling task participants had to choose between gambling for one of two options or for passing, in order to cumulate as many points as possible. We used percent signal change maps obtained during the four conditions of the gambling task as test set for the classifier trained on the gonogo percent signal change maps. This analysis again established high accuracies of discriminating between different age groups while performing a very different task, namely between 13 versus 17 year-olds (70.1\%), 13 versus 21 year-olds (78.1\%), and 17 versus 21 year-olds (82.9\%). This strengthens the hypothesis that developmental changes in functional activation concern a general pattern or principle, which seems to be largely independent of the specific cognitive task performed.

\section{ROI analyses}

Since the MVP classification analysis does not give information about the direction of age-related changes in brain activation, we performed ROI-based group analyses for a selection of regions that contributed to age group discrimination.

In two task-induced deactivated clusters that contributed to age group discrimination, i.e. ventral medial prefrontal and posterior cingulate cortices, PSC decreased with age (respectively $\mathrm{F}_{(2,53)}=8.05 ; \mathrm{p}=.001$ and $\mathrm{F}_{(2,54)}=8.40 ; \mathrm{p}=.001$ ). The 21 year-olds deactivated these regions more compared with 13 year-olds 
(respectively $\mathrm{p}=.001$ and $\mathrm{p}<.001$ ), with an intermediate position for the 17 yearolds which failed to reach significance (Figure 4A).

From the task positive areas that contributed to age group discrimination, we selected several regions from the different positive networks for ROI analysis (Figure 4B): viz supplementary motor area, postcentral gyrus, intraparietal sulcus, frontal eye field, anterior cingulate cortex and anterior insula. PSC in the two motor network areas increased with age, viz supplementary motor area $\left(\mathrm{F}_{(2}\right.$, 54) $=4.56 ; \mathrm{p}=.015)$ and postcentral gyrus $\left(\mathrm{F}_{(2,54)}=5.97 ; \mathrm{p}=.005\right)$. Postcentral gyrus was activated more by 21 and 17 year-olds compared with 13 year-olds (respectively $\mathrm{p}=.018$ and $\mathrm{p}=.009$ ), whereas only 21 year-olds activated supplementary motor area stronger compared with 13 year-olds ( $\mathrm{p}=.021)$, with an intermediate, non-significant position for the 17 year-olds. In the attention-related frontal eye field, the BOLD response increased with age as well $\left(\mathrm{F}_{(2,54)}=6.07\right.$; $\left.\mathrm{p}=.004\right)$, with higher PSC for 21 and 17 year-olds compared with 13 year-olds (respectively $\mathrm{p}=.009$ and $\mathrm{p}=.016$ ). In the second attention-related ROI, right intraparietal sulcus, the interaction of age and task condition was significant $\left(F_{(2,54)}=6.45\right.$; $\mathrm{p}=.003)$. The age effect was strongest during NOGO trials $\left(\mathrm{F}_{(2,54)}=9.18\right.$; $\mathrm{p}<.001)$, followed by GOoddball trials $\left(\mathrm{F}_{(2,54)}=3.94 ; \mathrm{p}=.025\right)$ and only borderline significant during $\mathrm{GO}$ trials $\left(\mathrm{F}_{(2,54)}=3.01 ; \mathrm{p}=.058\right)$. Furthermore, enhanced intraparietal activation during NOGO compared with GO trials ( $\mathrm{p}=.004)$ with an intermediate, non-significant position for GOoddball trails was only seen in 21 year-olds and not in 13 and 17 year-olds. The effect of age group on PSC failed to reach significance in the areas of the salience network, viz anterior cingulate cortex and anterior insula.

When repeating all these ROI analyses with reaction time variability as a covariate in the ANOVA, the described age group effects in all ROIs remained unchanged. This suggests that the age effects in BOLD response can not be explained by age differences in general task performance.

Thus, task-induced deactivation of default mode areas increased with age. At the same time, activation of motor and attention related areas increased, while there was no change in typical response control areas. Only in the attention-related right intraparietal sulcus the age effect was modulated by task conditions, with enhanced activation during inhibition only in 21 year-olds. 
A ROIs that overlapped with task negative responding areas

vmPFC (3 45 3)

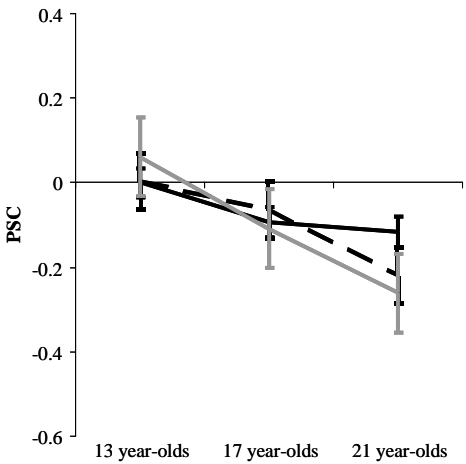

$\operatorname{PCC}(-6-4233)$

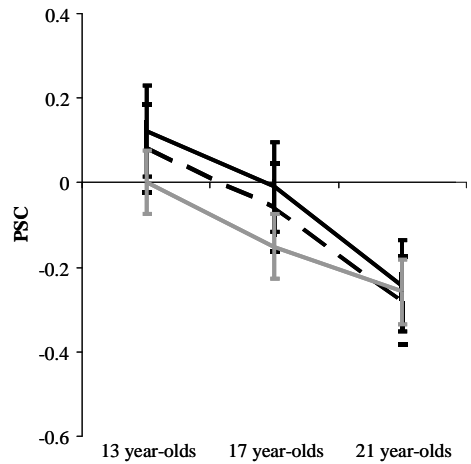

B ROIs that overlapped with task positive responding areas

SMA (0 -9 51)

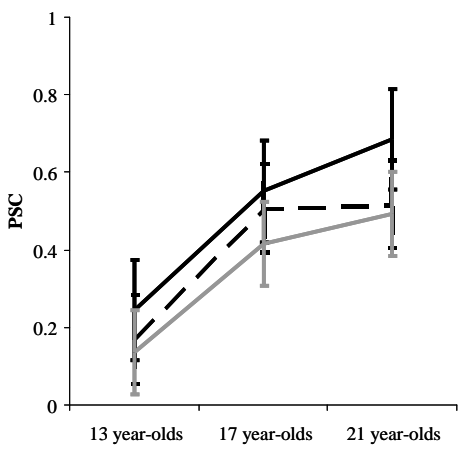

IPS (27 -66 33)

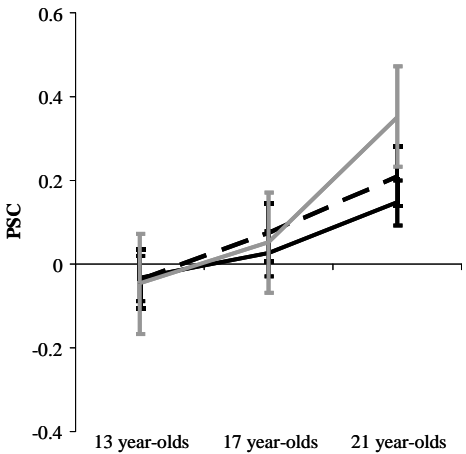

postcentral gyrus (-57 -24 39)



FEF (27 -15 57)




dACC (0 18 42)

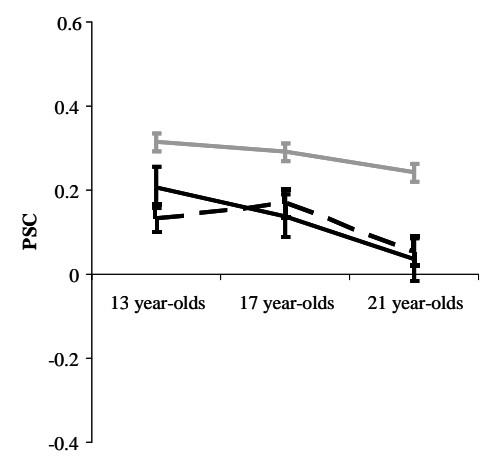

anterior insula (30 18 6)

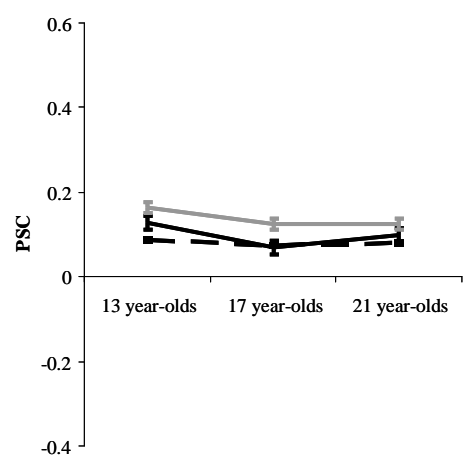

Figure 4: Percent signal changes in ROIs that contributed to age group classification. In each ROI mean percent signal changes are shown with error bars indicating standard error of the mean, separately for each trial type (GO(black), GOoddball (striped), NOGO (grey)). $\mathrm{vmPFC}=$ ventral medial prefrontal cortex; $\mathrm{PCC}=$ posterior cingulate cortex; SMA= supplementary motor area; IPS= intraparietal sulcus; $F E F=$ frontal eye field; $d A C C=$ dorsal part of anterior cingulate cortex.

\section{Discussion}

In the present study, multivariate pattern (MVP) classification analysis was applied for the first time in a developmental context with the intention to classify subjects based on functional activation maps. This multivariate approach was highly accurate in classifying different adolescent age groups based on their activation pattern during a simple gonogo task. The highest accuracies were obtained for discriminating 13 from 21 year-olds, followed by discriminating 13 from 17 year-olds, whereas accuracies for discriminating 17 from 21 year-olds did only reach significance when we used more optimal parameters for univariate and multivariate feature selection. These MVP classification results show that developmental changes are spatially distributed throughout the brain, affecting the responsiveness of a wide range of cortical structures. In addition, these findings suggest that the largest distributed developmental changes in functional brain activation take place before the age of 17. Age-related changes in functional activation after age 17 are less pronounced. The contributing voxels are still distributed across the brain, but their number is smaller. The voxels that contributed to age group classification were roughly confined to task-related areas, as they fell within or overlapped with brain areas that were either activated or deactivated during execution of the present gonogo task. Some voxels that contributed to age group classification are included in the default mode network (Dosenbach et al., 2007; Greicius et al., 2003), whereas other contribut- 
ing voxels can be classified within task positive functional networks, namely sensory-motor, attention, visual processing, salience and central executive networks. These functional networks have been described within resting state functional imaging and are consistent between subjects and sessions (Beckmann et al., 2005; Damoiseaux et al., 2006; De Luca et al., 2006; Dosenbach et al., 2007). The widespread age-related changes in functional responsiveness of both task positive and default mode brain networks correspond with maturational changes in functional connectivity between these areas (Fair et al., 2007, 2008; Kelly et al., 2009).

Moreover, we were able to show that these distributed age-distinctive patterns are not specific to the particular cognitive processes that are executed. The age-distinctive pattern based on signal changes during one cognitive process of the gonogo task (for instance, response execution in GO trials) is able to accurately predict age group based on signal changes during a different cognitive process of the gonogo task (for instance, inhibit a prepotent response in NOGO trials). Even stronger evidence for this general principle of functional brain maturation comes from the ability to generalize the age-distinctive pattern based on one cognitive task to a totally different task. The classifier trained on the gonogo data was able to accurately predict age group based on activation patterns during a complex and challenging gambling task. These results confirm our initial hypothesis that developmental changes in functional activation concern a general pattern, which is not specific to a particular cognitive function. This is in line with widespread maturational changes in brain anatomy in this developmental period (Giedd et al., 2009; Gogtay et al., 2004), which have general, functional a-specific implications for brain functioning. In addition, agerelated changes in functional connectivity have been shown between areas of distributed brain networks, that are activated during task execution regardless of the specific task demands (Fair et al., 2007, 2008).

With regard to default mode regions that contributed to age group classification, task-induced deactivation of these areas increased with age. For example, 21 year-olds deactivated ventral medial prefrontal and posterior cingulate cortices more during task execution compared with 13 year-olds, with an intermediate position for 17 year-olds. In age-affected areas that were associated with task positive networks, the BOLD response increased with age. The exact developmental trajectory differed somewhat between the different regions. In the postcentral gyrus and frontal eye field, PSC was higher in both 21 and 17 compared with 13 year-olds, whereas PSC in supplementary motor area was only enhanced in 21 compared with 13 year-olds. In addition, the age-related increase in right intraparietal activation was modulated by task response, with the greatest increase during nogo trials. Percent signal changes in anterior cingulate cortex and anterior insula, however, did not differ between age groups when examined in the ROI analyses. This suggests that prefrontal areas associated with the salience 
network (e.g. Sridharan et al., 2008) did contribute to age group discrimination in the MVP analysis, but this effect was not strong or reliably enough to be detected within these individual regions. These salience areas involved in cognitive control have among others the highest global brain connectivity, suggesting a role in the integration of brain activity in order to coordinate cognition and behavior (Cole et al., 2010). Networks such as the sensory-motor, visual and attentional networks are necessary for executing the gonogo task and are therefore connected by the cognitive control networks during task execution. Given that more effective long range connections are installed in the course of adolescence (Fair et al., 2007; Kelly et al., 2009), the connections between the cognitive control and other functional networks might improve with increasing age. This increased functional connectivity over longer ranges might underlie the age differences in activation of task positive areas observed in the present study.

In contrast to the high accuracy of classifying adolescent age groups revealed by the MVP classification analysis, the univariate voxel-wise analysis revealed few and small age-related clusters that only differed between 13 and 21 year-olds. The regions revealed by the GLM did, however, correspond with the regions highlighted in the MVP classification analysis. This finding underscores that an univariate voxel-wise approach lacks the sensitivity to reveal subtle and distributed age differences in brain activation. These relatively small age-related changes in percent signal change relative to the variability and noise in the measured data, fail to reach significance at the voxel level (Bray et al., 2009; De Martino et al., 2008).

In summary, the present study is the first to show that multivariate pattern classification analysis is highly accurate in classifying adolescents of 13 years of age versus respectively 17 and 21 year-olds based on their brain activation during a simple gonogo task. The MVP classification results show that functional maturation concerns subtle differences in responsiveness of both task positive and default mode regions widely distributed throughout the brain. More importantly, this age-distinctive pattern in brain activation is independent of the specific cognitive function executed, as it generalizes to different conditions of the gonogo task and even to a totally different gambling task. The present findings suggest that a multivariate approach might be more suited for studying developmental changes in brain activation than the traditional, univariate voxel-wise analysis. Furthermore, this study shows evidence for widespread changes in brain activation that are a-specific to cognitive functions, which suggests an alternative view on functional brain maturation that is in line with widespread maturational changes in anatomy and functional connectivity. 


\section{References}

Achenbach, T.M., Rescorla, L.A., 2001. Manual for the ASEBA school-age forms \& profiles. Burlington, VT: University of Vermont, Research Centre for Children, Youth and Families.

Achenbach, T.M., Rescorla, L.A., 2003. Manual for ASEBA adult forms and profiles. Burlington, VT: University of Vermont, Research Centre for Children, Youth and Families.

Ardila, A., Rosselli, M., Matute, E., Guajardo, S., 2005. The influence of the parents' educational level on the development of executive functions. Developmental Neuropsychology, 28(1), 539-560.

Aron, A.R., Poldrack, R.A., 2005. The cognitive neuroscience of response inhibition: Relevance for genetic research in attention-deficit/hyperactivity disorder. Biological Psychiatry, 57, 1285-1292.

Beckmann, C.F., DeLuca, M., Devlin, J.T., Smith, S.M., 2005. Investigations into resting state connectivity using independent component analysis. Philosophical Transactions of the Royal Society B, 360, 1001-1013.

Bennett, C.M., Miller, M.B., Wolford, G.L., 2009. Neural correlates of interspecies perspective taking in the post-mortem Atlantic Salmon: an argument for multiple comparisons correction. In: Organization for Human Brain Mapping Abstracts.

Blakemore, S.J., den Ouden, H., Choudhury, S., Frith, C., 2007. Adolescent development of the neural circuitry for thinking about intentions. Social Cognitive and Affective Neuroscience, 2(2), 130-139.

Booth, J.R., Burman, D.D., Meyer, J.R., Lei, Z., Trommer, B.L., Davenport, N.D., et al., 2003. Neural development of selective attention and response inhibition. NeuroImage, 20, 737-751.

Bray, S., Chang, C., Hoeft, F., 2009. Applications of multivariate pattern classification analyses in developmental neuroimaging of healthy and clinical populations. Frontiers in Human Neuroscience, 3, 1-12.

Buchsbaum, B.R., Greer, S., Chang, W., Berman, K.F., 2005. Meta-analysis of neuroimaging studies of the Wisconsin card-sorting task and component processes. Human Brain Mapping, 25, 35-45.

Bunge, S.A., Dudukovic, N.M., Thomason, M.E., Vaidya, C.J., Gabrieli, J.D.E., 2002. Immature frontal lobe contributions to cognitive control in children: Evidence from fMRI. Neuron, 33, 301-311.

Casey, B.J., Getz, S., Galvan, A., 2008. The adolescent brain. Developmental Review, 28, 6277.

Casey, B.J., Trainor, R.J., Orendi, J.L., Schubert, A.B., Nystrom, L.E., Giedd, J.N., et al., 1997. A developmental functional MRI study of prefrontal activation during performance of a go-no-go task. Journal of Cognitive Neuroscience, 9(6), 835-847.

Chikazoe, J., Jimura, K., Asari, T., Yamashita, K., Morimoto, H., Hirose, S., et al., 2009. Functional dissociation in right inferior frontal cortex during performance of go/no-go task. Cerebral Cortex, 19, 146-152.

Cole, M.W., Pathak, S., Schneider, W., 2010. Identifying the brain's most globally connected regions. NeuroImage, 49, 3132-3148.

Cole, M.W., Schneider, W., 2007. The cognitive control network: Integrated cortical regions with dissociable functions. NeuroImage, 37, 343-360.

Corbetta, M., Shulman, G.L., 2002. Control of goal-directed and stimulus-driven attention in the brain. Nature Reviews Neuroscience, 3, 201-215. 
Dahl, R.E., 2004. Adolescent brain development: a period of vulnerabilities and opportunities. Keynote address. Annals of the New York Academy of Sciences, 1021, 1-22.

Damoiseaux, J.S., Rombouts, S.A.R.B., Barkhof, F., Scheltens, P., Stam, C.J., Smith, S.M., et al., 2006. Consistent resting-state networks across healthy subjects. Proceedings of the National Academy of Sciences U.S.A., 103(37), 13848-13853.

De Luca, M., Beckmann, C.F., De Stefano, N., Matthews, P.M., Smith, S.M., 2006. fMRI resting state networks define distinct modes of long-distance interactions in the human brain. NeuroImage, 29, 1359-1367.

De Martino, F., Valente, G., Staeren, N., Ashburner, J., Goebel, R., Formisano, E., 2008. Combining multivariate voxel selection and support vector machines for mapping and classification of fMRI spatial patterns. NeuroImage, 43, 44-58.

Derrfuss, J., Brass, M., von Cramon, D.Y., 2004. Cognitive control in the posterior frontolateral cortex: evidence from common activations in task coordination, interference control, and working memory. NeuroImage, 23, 604-612.

Directoraat-Generaal voor de Arbeidsvoorziening (1989). Handleiding voor de functieanalyse [Function analyses manual]. Den Haag: SDU uitgeverij.

Dosenbach, N.U.F, Fair, D.A., Miezin, F.M., Cohen, A.L., Wenger, K.K., Dosenbach, R.A.T., et al., 2007. Distinct brain networks for adaptive and stable task control in humans. Proceedings of the National Academy of Sciences U.S.A., 104(26), 11073-11078.

Duncan, J., Owen, A.M., 2000. Common regions of the human frontal lobe recruited by diverse cognitive demands. Trends in Neurosciences, 23(10), 475-483.

Dunn, L.M., Dunn, L.M., 2005. Peabody Picture Vocabulary Test-III-NL. Handleiding [Manual]. Amsterdam: Harcourt Assessment B.V.

Durston, S., Thomas, K.M., Yang, Y., Ulu, A.M., Zimmerman, R.D., Casey, B.J., 2002. A neural basis for the development of inhibitory control. Developmental Science, 5(4), F9-F16.

Fair, D.A., Cohen, A.L., Dosenbach, N.U.F., Church, J.A., Miezin, F.M., Barch, D.M., et al., 2008. The maturing architecture of the brain's default network. Proceedings of the National Academy of Sciences U.S.A., 105(10), 4028-4032.

Fair, D.A., Dosenbach, N.U.F., Church, J.A., Cohen, A.L., Brahmbhatt, S., Miezin, F.M., et al., 2007. Development of distinct control networks through segregation and integration. Proceedings of the National Academy of Sciences U.S.A., 104, 13507-13512.

Fox, M.D., Corbetta, M., Snyder, A.Z., Vincent, J.L., Raichle, M.E., 2006. Spontaneous neuronal activity distinguishes human dorsal and ventral attention systems. Proceedings of the National Academy of Sciences U.S.A., 103(26), 10046-10051.

Friston, K.J., Williams, S., Howard, R., Frackowiak, R.S.J., Turner, R., 1996. Movementrelated effects in fMRI time-series. Magnetic Resonance in Medicine, 35(3), 346-355.

Garavan, H., Hester, R., Murphy, K., Fassbender, C., Kelly, C., 2006. Individual differences in the functional neuroanatomy of inhibitory control. Brain Research, 1105, 130-142.

Genovese, C.R., Lazar, N.A., Nicols, T., 2002. Thresholding of statistical maps in functional neuroimaging using the false discovery rate. NeuroImage, 15, 870-878.

Giedd, J.N., 2004. Structural magnetic resonance imaging of the adolescent brain. Annals of the New York Academy of Sciences, 1021, 77-85.

Giedd, J.N., Lalonde, F.M., Celano, M.J., White, S.L., Wallace, G.L., Lee, N.R. et al., 2009. Anatomical brain magnetic resonance imaging of typically developing children and adolescents. Journal of the American Academy of Child and Adolescent Psychiatry, 48(5), 465-470.

Giorgio, A., Watkins, K.E., Chadwick, M., James, S., Winmill, L., Douaud, G., et al., 2010. Longitudinal changes in grey and white matter during adolescence. NeuroImage, 49, 94103. 
Gogtay, N., Giedd, J.N., Lusk, L., Hayashi, K.M., Greenstein, D., Vaituzis, A.C., et al., 2004. Dynamic mapping of human cortical development during childhood through early adulthood. Proceedings of the National Academy of Sciences U.S.A., 101(21), 81748179.

Greicius, M.D., Krasnow, B., Reiss, A.L., Menon, V., 2003. Functional connectivity in the resting brain: A network analysis of the default mode hypothesis. Proceedings of the National Academy of Sciences U.S.A., 100(1), 253-258.

Guyon, I., Weston, J., Barnhill, S., Vapnik, V., 2002. Gene selection for cancer classification using support vector machines. Machine Learning, 46, 389-422.

Hampshire, A., Chamberlain, S.R., Monti, M.M., Duncan, J., Owen, A.M., 2010. The role of the right inferior frontal gyrus: inhibition and attentional control. NeuroImage, 50(3), 1313-1319.

Huizinga, M., Dolan, C. V., van der Molen, M.W., 2006. Age-related changes in executive functions: Developmental trends and a latent variable analysis. Neuropsychologia, 44, 2017-2036.

Johnstone, T., Ores Walsh, K.S., Greischar, L.L., Alexander, A.L., Fox, A.S., Davidson, R.., et al., 2006. Motion correction and the use of motion covariates in multiple-subject fMRI analysis. Human Brain Mapping, 27, 779-788.

Kelly, A.M.C., Di Martino, A., Uddin, L.Q., Shehzad, Z., Gee1, D.G., Reiss, P.T., et al., 2009. Development of anterior cingulate functional connectivity from late childhood to early adulthood. Cerebral Cortex, 19, 640-657.

Keulers, E.H.H., Stiers, P., Jolles, J., 2010 Developmental changes between ages 13-21 in the extent and magnitude of the BOLD response during decision making. NeuroImage, in press.

Klenberg, L., Korkman, M., Lahti-Nuuttila, P., 2001. Differential development of attention and executive functions in 3- to 12-year-old Finnish children. Developmental Neuropsychology, 20(1), 407-428.

Luna, B., Garver, K.E., Urban, T.A., Lazar, N.A., Sweeney, J.A., 2004. Maturation of cognitive processes from late childhood to adulthood. Child Development, 75(5), 1357-1372.

Mayer, A.R., Franco, A.R., Ling, J., Canive, J.M., 2007. Assessment and quantification of head motion in neuropsychiatric functional imaging research as applied to schizophrenia. Journal of the International Neuropsychological Society, 13, 839-845.

Mennes, M., Stiers, P., Lagae, L., Van den Bergh, B., 2006. Long-term cognitive sequelae of antenatal maternal anxiety: involvement of the orbitofrontal cortex. Neuroscience and Biobehavioral Reviews, 30, 1078-1086.

Mourao-Miranda, J., Bokde, A.L.W., Born, C., Hampel, H., Stetter, M., 2005. Classifying brain states and determining the discriminating activation patterns: Support Vector Machine on functional MRI data. NeuroImage, 28, 980-995.

Poldrack, R.A., Mumford, J.A., 2009. Independence in ROI analysis: where is the voodoo? Social Cognitive and Affective Neuroscience, 4, 208-213.

Raven, J., Raven, J.C., Court, J.H., 1998. Manual for Raven’s Progressive Matrices and Vocabulary Scales. Section 1: General Overview. Harcourt Assessment, San Antonio TX.

Rubia, K., Smith, A.B., Taylor, E., Brammer, M., 2007. Linear age-correlated functional development of right inferior fronto-striato-cerebellar networks during response inhibition and anterior cingulate during error-related processes. Human Brain Mapping, 28, 1163-1177.

Rubia, K., Smith, A.B., Woolley, J., Nosarti, C., Heyman, I., Taylor, E., et al., 2006. Progressive increase of frontostriatal brain activation from childhood to adulthood during event-related tasks of cognitive control. Human Brain Mapping, 27, 973-993. 
Ryali, S., Supekar, K., Abrams, D.A., Menon, V., 2010. Sparse logistic regression for whole brain classification of fMRI data. Neuroimage, 51(2), 752-764.

Simmonds, D.J., Pekar, J.J., Mostofsky, S.H., 2008. Meta-analysis of go/no-go tasks demonstrating that fMRI activation associated with response inhibition is task- dependent. Neuropsychologia, 46, 224-232.

Slifer, K.J., Cataldo, M.F., Cataldo, M.D., Llorente, A.M., Gerson, A.C., 1993. Behavior analysis of motion control for pediatric neuro-imaging. Journal of Applied Behavior Analysis, 269, 469-470.

Sridharan, D., Levitin, D.J., Menon, V., 2008. A critical role for the right fronto-insular cortex in switching between central-executive and default-mode networks. Proceedings of the National Academy of Sciences U.S.A., 105(34), 12569-12574.

Steinberg, L., 2008a. A social neuroscience perspective on adolescent risk-taking. Developmental Review, 28, 78-106.

Steinberg, L., Albert, D., Cauffman, E., Banich, M., Graham, S., Woolard, J., 2008b. Age differences in sensation seeking and impulsivity as indexed by behavior and self report: Evidence for a dual systems model. Developmental Psychology, 44(6), 1764-1778.

Stiers, P., Mennes, M., Sunaert, S., 2010. Distributed task coding throughout the multiple demand network of the human frontal-insular cortex, NeuroImage, 52(1), 252-262.

Stuss, D.T., Murphy, K.J., Binns, M.A., Alexander, M.P., 2003. Staying on the job: the frontal lobes control individual performance variability. Brain, 126, 2363-2380.

Suykens, J.A.K., Van Gestel, T., De Barbanter, J., De Moor, B., Vanderwalle, J., 2002. Least Squares Support Vector Machines. World Scientific Publishing.

Talairach, J., Tournoux, P., 1988. Co-planar stereotaxic atlas of the human brain. Thieme Medical Publishers Inc, New York.

Tamm, L., Menon, V., Reiss, A.L., 2002. Maturation of brain function associated with response inhibition. Journal of the American Academy of Child \& Adolescence Psychiatry, 41(10), 1231-123.

Van Leijenhorst, L., Gunther Moor, B., Op de Macks, Z.A., Rombouts, S.A.R.B., Westenberg, P.M., Crone, E.A., 2010. Adolescent risky decision-making: Neurocognitive development of reward and control regions. Neuroimage, 51(1), 345-355.

Yoo, S., Choi, B., Juh, R., Pae, C., Lee, C., 2005. Head motion analysis during cognitive fMRI examination: Application in patients with schizophrenia. Neuroscience Research, 53, 84-90. 
Supplementary Table 1: Overview of task positive and negative responding areas during a pooled gonogo task contrast independent of age group

\begin{tabular}{|c|c|c|c|c|c|c|}
\hline Brain area & & $\begin{array}{l}\text { TAL } \\
x \quad y \quad z \\
\end{array}$ & Size & T value & $\begin{array}{l}\text { Dosenbach et } \\
\text { al., } 2007\end{array}$ & $\begin{array}{l}\text { Sridharan et } \\
\text { al., } 2008\end{array}$ \\
\hline \multicolumn{7}{|c|}{ POSITIVE RESPONDING AREAS } \\
\hline supplementary motor area & $\mathrm{L}$ & $-9-648$ & 320 & 11.80 & & \\
\hline anterior cingulate cortex & $\mathrm{R}$ & 61233 & & 7.49 & -11046 & 43030 \\
\hline anterior insula & $\mathrm{R}$ & $\begin{array}{l}30129 \\
42123\end{array}$ & 60 & $\begin{array}{l}6.84 \\
6.62\end{array}$ & 36164 & $3725-4$ \\
\hline anterior insula & $\mathrm{L}$ & -33915 & 28 & 6.54 & -35144 & $-3424-6$ \\
\hline posterior insula & $\mathrm{L}$ & 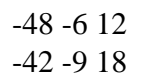 & 29 & $\begin{array}{l}8.18 \\
5.20\end{array}$ & & \\
\hline inferior frontal sulcus & $\mathrm{R}$ & 332730 & 18 & 5.54 & 432234 & 451645 \\
\hline thalamus & $\mathrm{L}$ & $-15-189$ & 107 & 9.23 & $-12-157$ & \\
\hline putamen & & $\begin{array}{l}-2433 \\
-18315\end{array}$ & & $\begin{array}{l}7.56 \\
6.17\end{array}$ & & \\
\hline thamalus & $\mathrm{R}$ & $9-312$ & 32 & 6.26 & $10-158$ & \\
\hline putamen & & 1833 & & 6.20 & & \\
\hline postcentral gyrus & $\mathrm{L}$ & $\begin{array}{lll}-42 & -21 & 54 \\
-54 & -21 & 45 \\
-51 & -36 & 48\end{array}$ & 207 & $\begin{array}{l}10.19 \\
8.89 \\
5.73\end{array}$ & & \\
\hline fusiform gyrus & $\mathrm{L}$ & $\begin{array}{lll}-39 & -51 & -21 \\
-39 & -60 & -15 \\
-27 & -78 & -9\end{array}$ & 138 & $\begin{array}{l}11.14 \\
9.20 \\
6.65\end{array}$ & $-32-66-29$ & \\
\hline fusiform gyrus & $\mathrm{R}$ & $\begin{array}{l}27-45-21 \\
33-63-9 \\
18-42-18\end{array}$ & 381 & $\begin{array}{l}10.65 \\
10.28 \\
9.92\end{array}$ & $31-61-29$ & \\
\hline lingual gyrus & $\mathrm{R}$ & $21-843$ & 24 & 11.50 & $8-824$ & \\
\hline middle occipital gyrus & $\mathrm{L}$ & $-30-813$ & 11 & 7.27 & & \\
\hline NEGATIVE RESPONDIN & AR & EAS & & & & \\
\hline superior frontal sulcus & $\mathrm{L}$ & -24948 & 5 & 5.26 & & \\
\hline inferior frontal sulcus & $\mathrm{L}$ & -512715 & 10 & 5.42 & & \\
\hline posterior insula & $\mathrm{R}$ & $\begin{array}{lll}33 & -18 & 21 \\
42 & -18 & 9 \\
33 & -15 & 12\end{array}$ & 318 & $\begin{array}{l}10.50 \\
10.05 \\
7.79\end{array}$ & & \\
\hline superior parietal lob & & $21-3960$ & 913 & 10.61 & & \\
\hline postcentral sulcus & & $21-2457$ & & 8.60 & & \\
\hline precentral sulcus & & $33-2139$ & & 8.53 & & \\
\hline $\begin{array}{l}\text { posterior cingulate cortex/ } \\
\text { anterior calcarine sulcus }\end{array}$ & $\mathrm{L}$ & $\begin{array}{llll}-2 & -57 & 15 \\
-12 & -84 & 21 \\
-18 & -72 & 24\end{array}$ & 234 & $\begin{array}{l}6.79 \\
6.52 \\
6.38\end{array}$ & $-11-5713$ & $7-4333$ \\
\hline lingual gyrus & $\mathrm{R}$ & $\begin{array}{l}3-663 \\
12-6618 \\
3-6012\end{array}$ & 236 & $\begin{array}{l}6.87 \\
6.73 \\
3.60\end{array}$ & $8-824$ & \\
\hline parahippocampal gyrus & $\mathrm{L}$ & $\begin{array}{lll}-30 & -36 & -9 \\
-21 & -39 & -9 \\
-27 & -48 & 6\end{array}$ & 101 & $\begin{array}{l}7.17 \\
6.98 \\
5.11\end{array}$ & $-25-44-12$ & \\
\hline parahippocampal gyrus & $\mathrm{R}$ & $21-36-6$ & 7 & 5.73 & $25-44-12$ & \\
\hline parahippocampal gyrus & $\mathrm{R}$ & $24-30-12$ & 5 & 5.26 & & \\
\hline superior temporal gyrus & $\mathrm{L}$ & $-48-246$ & 79 & 8.22 & & \\
\hline
\end{tabular}


Note. The whole brain GLM results described here are significant at the family wise error corrected level $(\mathrm{F}<.05 ; \mathrm{F}=24.64$; voxels $\geq 5)$. In the last two columns the present results are compared with two studies that described areas within task positive networks as well as the default mode network (Dosenbach et al., 2007; Sridharan et al., 2008). Size in number of voxels. $\mathrm{TAL}=$ Talairach coordinates; $\mathrm{R}=$ right hemisphere; $\mathrm{L}=$ left hemisphere; $\mathrm{VMP}=$ ventral medial nucleus of the thalamus. 

Chapter

Developmental fMRI studies: should cortisol reactivity be considered as confounder?

Esther H.H. Keulers, Peter Stiers, Nancy A. Nicolson and Jelle Jolles Under review. 


\begin{abstract}
Functional Magnetic Resonance Imaging (fMRI) is a frequently used tool in examining the neural substrate of cognitive processes, but is sometimes experienced as stressful. Stressors trigger the secretion of cortisol, which has been shown to influence cognition as well as brain functioning. The present study aimed to examine whether cortisol reactivity to fMRI scanning differed between young and old adolescents and investigated the association between cortisol and brain activation during a gambling task. Cortisol levels were elevated pre relative to post imaging in all participants, suggesting anticipatory stress. A subgroup of adolescents was characterized by an enhanced cortisol output during the MRI session, irrespective of age. Compared with non-responders, these stress responders showed decreased activation in the inferior parietal cortex, which is related to attentional processes. Furthermore, associations between cortisol and the BOLD response were observed only in young as opposed to old adolescents, suggesting an age-dependent effect of cortisol on brain activation. The present results therefore suggest that cortisol reactivity to fMRI scanning should be considered as a confounder and should be incorporated in analysing fMRI data, especially in developmental fMRI studies.
\end{abstract}




\section{Introduction}

Magnetic Resonance Imaging (MRI) of the head has become a valuable and frequently used tool for both diagnostic and research purposes. Although the procedure is non-invasive, 5 to $30 \%$ of subjects undergoing (f)MRI scanning have reported psychological distress and anxiety (Melendez and McCranck, 1993). These reactions range from apprehension caused by spatial restriction in the scanner to more severe panic or claustrophobic reactions. There are indications that psychological distress in relation to (f)MRI scanning might affect the quality of the images and interferes with task performance (Dantendorfer et al., 1997; Eatough et al., 2009; Melendez and McCranck, 1993).

Subjects who experience (f)MRI scanning as stressful may likely manifest a neuroendocrine response during the MRI session. Stressors activate the hypothalamic-pituitary-adrenocortical (HPA) axis, particularly in situations characterized by novelty, unpredictability, and low perceived control (Dickerson and Kemeny, 2004), and this is generally the case in an (f)MRI experiment. Activation of the HPA axis triggers the secretion of cortisol. Marked inter-individual differences have been shown in both baseline cortisol levels and stress reactivity (Kirschbaum and Hellhammer, 1994). In addition, subgroups of subjects with similar endocrinological patterns have been differentiated, for example showing either increased or decreased cortisol in response to stressors (Pruessner et al., 2003, 2008). Although elevated levels of cortisol in relation to (f)MRI scanning have been shown in both adults (Tessner et al., 2006) and adolescents (Eatough et al., 2009), age groups have never been compared directly. Being subjected to (f)MRI scanning might be more stressful for children and adolescents compared with adults, as their ability to regulate emotions and stress is still developing (Nelson et al., 2005). Likewise, there are no data available yet with respect to individual differences in cortisol reactivity to (f)MRI scanning within different age groups.

Cortisol has been shown to influence cognition and brain function. Cortisol can easily cross the blood-brain barrier and binds to receptors in the brain, with a preference for limbic system components including hippocampus, amygdala and prefrontal cortex (Lupien et al., 2007). Cortisol levels have been shown to influence cognition according to an inverted U-shaped curve (de Kloet et al., 1999). Decreased as well as increased levels of cortisol due to either pharmacological manipulations or laboratory stressors impaired cognitive functions, such as declarative and working memory (e.g., de Quervain et al., 2000; Kirschbaum et al., 1996; Lupien et al., 2002; Newcomer et al., 1999). Several neuroimaging studies have examined the neural correlates of stress by using psychological stress paradigms (e.g., the Montreal Imaging Stress Task (Dedovic et al., 2005)). 
However, the results have been divergent, and overall interpretation is hampered by methodological differences (Dedovic et al., 2009a, 2009b). The most consistent finding is the decreased activation in medial and orbital prefrontal cortices during these stress paradigms (Kern et al., 2008; Pruessner et al., 2008; Wang et al., 2005). Furthermore, increased cortisol levels have been associated with decreased activation in limbic structures, such as the hippocampus (de Quervain et al., 2003; Pruessner et al., 2008). Positive associations have been established between cortisol levels and activation in ventrolateral prefrontal, anterior cingulate, and parietal areas (Kern et al., 2008; Wang et al., 2005, 2007; Weerda et al., 2010).

In summary, individual differences in cortisol reactivity to (f)MRI scanning might confound the cognitive and neuroimaging results, especially in studies comparing age groups. Kukolja et al. (2008) showed that the association of cortisol with both behavioral performance and brain activation during a spatial working memory task was different in young (19-29 years) versus old (52-71 years) adults. To our knowledge, no such data is available for developing age groups. Therefore, the present study examined cortisol changes in relation to fMRI scanning in two adolescent age groups to determine whether cortisol reactivity to fMRI is age-dependent. The levels of salivary cortisol were assessed before, during and after the scan session in 13 year-old and 17 year-old males. In addition to cortisol reactivity, this study examined the association between cortisol and the BOLD response during performance of a gambling task.

\section{Methods}

Participants and procedure

A subsample of participants from a larger study examining developmental changes in brain activation during various cognitive tasks (Keulers et al., 2010) provided salivary cortisol measures. All participants had normal or corrected-tonormal vision, were free from psychiatric or neurological abnormalities, were screened for MRI contra-indications, and did not use medication that could influence cognitive functioning or salivary cortisol. Written informed consent was obtained from all participants and their parents. Before the scan session participants attended a training session, which served to familiarize them with the scanning environment and procedure as well as to have them practice the experimental tasks using a mock scanner. Participants received travel expenses and a fee of 25 euros. The study was approved by the ethical committee of the Faculty Psychology and Neuroscience of Maastricht University. From the total of 24 participants included in this study, two participants were excluded due to excessive head motion (see section Statistical analyses - Imaging data - Pre- 
processing for details). This resulted in a final sample of 22 right-handed males, divided into two age groups: young adolescents $(\mathrm{N}=11$, mean age $=12.9$, $\mathrm{SD}=0.3)$ and old adolescents $(\mathrm{N}=11$, mean age $=16.8, \mathrm{SD}=0.3)$.

\section{Measurements}

\section{Salivary cortisol}

A total of five saliva samples were obtained, of which the first two were used to define a baseline cortisol level. The baseline samples were obtained at approximately the same time as the start of the scan session $\left(\mathrm{F}_{(1,20)}=0.70 ; \mathrm{p}=.51\right)$ on two preceding days: halfway through the practice session (measure 1) and at home (measure 2). The third sample was collected at the start of the scan session (measure 3). A fourth saliva sample was obtained in the scanner after the participant had finished the first functional run (measure 4). The final sample was collected immediately after the participant had finished the scan session (measure 5). Measures 4 and 5 were collected 13.7 ( $\mathrm{SD}=2.2$ ) and 76.5 ( $\mathrm{SD}=4.2$ ) minutes, respectively, after the participant had entered the bore of the magnet. All saliva samples were collected in the mid and late afternoon to control for diurnal fluctuations in salivary cortisol level. There was a slight difference in the start time of the scan session between young (mean time 16:37 ( $\mathrm{SD}=0: 52)$ ) and old (mean time 15:15 $(\mathrm{SD}=1: 35)$ ) adolescents $\left(\mathrm{T}_{(21)}=2.50 ; \mathrm{p}=.02\right)$. To exclude any possible influence of this small time difference, the analyses were performed with and without the time of cortisol measure 3 as a covariate.

Salivary cortisol was collected with a cotton dental roll, which was stored in a plastic tube (Salivette; Sarstedt, Etten-Leur, the Netherlands). Subjects were asked not to eat or smoke 1.5 hour prior to saliva sampling. They were told to chew lightly on the swab and to keep it fully in their mouth for 2 minutes. For the cortisol sampling at home, subjects were instructed to write down the exact time of the measurement on a form that also contained the specific instructions mentioned above. Samples were stored in subjects' home freezers until transport to the lab. In the lab, uncentrifuged samples were frozen at $-20^{\circ} \mathrm{C}$ until analysis.

Salivary cortisol levels were determined in duplicate by direct radioimmunoassay using 125I-cortisol and antiserum made against the 3-CMO-BSA conjugate by Dr. J. Sulon, University of Liege, Belgium. The lower detection limit of the assay was $0.3 \mathrm{nmol} / \mathrm{l}$, with a mean intra-assay coefficient of variation of $4.8 \%$. All samples from an individual subject were analyzed in the same assay to reduce sources of variability.

\section{Gambling task}

Participants performed a gambling task in which they could either gamble or pass in order to earn as many points as possible (Mennes et al., 2008; Stiers et al., 2010). In each trial a horizontal bar divided into two colored parts was pre- 
sented (range from 5\%-95\% to 50\%-50\%), indicating the probability of an imaginary token being hidden underneath. Participants could guess under which part a token was hidden by pressing the corresponding left or right button. Depending on the correctness of their choice, points could be won or lost. The points that could be won were indicated above the bar (range 10-100). The points that could be lost were presented below the bar (range 0-100), with the most ambiguous proportions (50\%-50\%) coupled with the highest losses (80100 points). Participants could also choose to pass by withholding their response, resulting in a small reward of 20 points. All participants started with 100 points. Feedback about the trial and an update of their total score was provided in $67 \%$ of the trials, in order to disentangle the effects related to feedback processing from those related to the decision making process.

The behavioral results from a previous study with this task (Mennes et al., 2008) were used to delineate four trial types along two dimensions. Task difficulty (exogenous vs endogenous decision making) and task response (gambling vs passing) were manipulated, leading to easy, exogenous trials in which participants could either gamble (exo GAMBLE) or pass (exo PASS) and to difficult, endogenous trials in which participants could either gamble (endo GAMBLE) or pass (endo PASS). These trial conditions were not relevant for the current analyses. The task comprised three runs of 7.3 minutes and 50 trials each. The bar was presented for 3.5 seconds and was, in 2/3 of the trials, followed 0.22.0 seconds later by feedback for 1.7 seconds. The interval between the end of the feedback and the start of a new trial varied between 1.0-4.0 seconds.

Statistical analyses

\section{Cortisol data}

The statistical package SPSS 16.0 was used for the analyses. Of all 110 salivary cortisol measures, measure 3 was missing for one young adolescent and measure 5 was missing for another young adolescent. In order to include these two young adolescents in the analyses, the missing cortisol values were replaced by the mean cortisol value of the particular measure for that age group (Tabachnick and Fidell, 2001). Natural logarithmic transformations were applied before analyses to meet the criteria for normality. To determine cortisol changes over time and/or changes due to age, an analysis of variance (ANOVA) with salivary cortisol measures (5 levels) as within-subject variable and age group (2 levels) as between-subject variable was conducted. Furthermore, to examine effects of cortisol on behavioral measures as well as on brain activation we determined two metrics of cortisol. The Area Under the Curve (AUC) for the cortisol values before, during and after fMRI scanning was calculated, with respect to both ground and increase using the trapezoid formulas as suggested by Pruessner et al. (2003). The AUC with respect to ground $\left(A_{U C}\right)$ indicates the total amount 
of cortisol output during the fMRI experiment. The AUC with reference to increase $\left(A \mathrm{AC}_{\mathrm{I}}\right)$ indicates cortisol changes over time, with positive values representing an increase over time and negative values representing a decrease with respect to the pre-imaging cortisol level. Natural logarithmic transformations were applied on both AUC values to meet criteria for normality, after a linear translation had been applied to the $\mathrm{AUC}_{\mathrm{I}}$ values to deal with negative values. The advantages of the AUC values are the merging of repeated measures and taking into account different time intervals between measures. Two independent sample T-tests with age group (2 levels) as between-subject variable were conducted to examine age differences in the $\mathrm{AUC}_{\mathrm{G}}$ and $\mathrm{AUC}_{\mathrm{I}}$. All analyses were repeated with the time of cortisol measure 3 included in the model as a covariate.

Given large inter-individual differences in cortisol levels and changes over time, we defined subgroups of participants with similar endocrinological patterns by using cluster analysis (k-means method) on the AUC values (Pruessner et al., 2003). Based on the $\mathrm{AUC}_{\mathrm{I}}$ we could not differentiate clusters of participants, whereas based on the $\mathrm{AUC}_{\mathrm{G}}$ two clusters were revealed. This resulted in seven responders (two young adolescents) and fifteen non-responders (nine young adolescents). Compared with non-responders, responders were characterized by a higher total cortisol output during fMRI scanning. The responders did not differ from the non-responders in either age $\left(\mathrm{T}_{(21)}=-1.42 ; \mathrm{p}=.18\right)$ or in timing of the cortisol measure $\left(\mathrm{T}_{(21)}=0.38 ; \mathrm{p}=.71\right)$. Next, this new group variable (responders vs non-responders based on $\mathrm{AUC}_{\mathrm{G}}$ ) was used as between-subject variable in an ANOVA, with salivary cortisol measures (5 levels) as within-subject variable.

To examine the influence of cortisol on cognitive performance, correlation analyses were performed between the two AUC values and all behavioral measures on the gambling task. Furthermore, differences between responders and non-responders in total number of points, number of exo PASS errors (i.e., failure to withhold response by a gain of 10 or 20 points) and negative feedback were analysed with a non-parametric Mann-Whitney test due to non-normality. Reaction times were analyzed with an ANOVA with task difficulty (2 levels: exo GAMBLE vs endo GAMBLE trials in which a response was given) as within-subject variable and group (2 levels: responders vs non-responders) as between-subject variable.

Imaging data

Acquisition

A Siemens MAGNETOM Allegra 3T MRI head-only scanner was employed. Head motion was constrained by the use of foam padding. A total of 32 axial slices covering the whole brain including the cerebellum were imaged by using a T2*-weighted gradient echo planner pulse sequence $(\mathrm{TR}=2000 \mathrm{~ms}$, TE $=30$ 
ms, $\mathrm{FA}=90, \mathrm{FOV}=224$, slice thickness $=4 \mathrm{~mm}$, matrix size $=64 \times 64$, flip angle $=90^{\circ}$ ). Voxel size was $3.5 \times 3.5 \times 4$ mm. A T1-weighted anatomical scan was acquired to aid with spatial normalization ( $\mathrm{TR}=2250 \mathrm{msec}$, TE $=2.6 \mathrm{msec}$, flip angle $=9^{\circ}, \mathrm{FOV}=256 \mathrm{~mm}$, slice thickness $=1 \mathrm{~mm}$, matrix size $=256 \mathrm{x}$ 256 , number of slices $=192$ ). Voxel size was 1 x 1 x $1 \mathrm{~mm}$. Slice scanning order was ascending interleaved.

\section{Preprocessing}

Data were preprocessed using BrainVoyager QX, version 1.9 (Brain Innovation, Maastricht, The Netherlands). Images were corrected for slice scan time differences, followed by rigid body motion correction, high-pass temporal filtering, and spatial data smoothing using a Gaussian kernel with a $4 \mathrm{~mm}$ full width at half maximum. Functional data were coregistered with the anatomical volume using rigid body transformation and manual adjustments to obtain optimal fit by visual inspection, if necessary. Subsequently, both functional and structural volumes were transformed into standard stereotaxic space using Talairach normalization.

To control for the confounding effect of head motion we applied two corrections to our data (Friston et al., 1996; Johnstone et al., 2006). Firstly, head motion within one scan may distort the measured fMRI signal by spatial misallocation. Therefore, we identified scans during which head motion exceeded a particular threshold, viz absolute motion difference between successive scans in $\mathrm{z}$ direction greater than $0.4 \mathrm{~mm}\left(1 / 10^{\text {th }}\right.$ of the voxel size), and rotation around the $\mathrm{x}$ dimension greater than 0.26 degrees (angle corresponding to $0.4 \mathrm{~mm} \mathrm{z}$ displacement of frontopolar voxels, assuming the rotation point in middle of brain is $88 \mathrm{~mm}$ from the anterior end of the brain frontal pole (Talairach \& Tournoux, 1988)). Task trials (taking into account the hemodynamic response function delay) cause signal intensity changes with peak BOLD values 5 to 6 seconds after their occurrence. These signal changes may overlap in time with head motion a few scans later. Therefore, trials in a preceding time window of 1 to 8 seconds of the identified scan were modeled as an additional event of no interest (Keulers et al., 2010). Because this procedure reduces the number of trials available in the events of interest, we excluded one young adolescent and one old adolescent with more than $25 \%$ of their trials discarded. After applying this procedure, the age groups did not differ on the two most affected motion parameters for the remaining scans (Mayer et al., 2007; Yoo et al., 2005), namely translation in the $\mathrm{z}$ direction $\left(\mathrm{Tz} ; \mathrm{F}_{(1,20)}=.003 ; \mathrm{p}=.959\right)$ and rotation in the $\mathrm{x}$ direction $\left(\mathrm{Rx} ; \mathrm{F}_{(1,20)}=2.73 ; \mathrm{p}=.114\right)$. Second, we included the six motion parameters as covariates of no interest in our general linear model to model signal intensity changes due to head motion (Friston et al., 1996; Johnstone et al., 2006). 
Analyses

Statistical analyses were performed on individual participants data by using the general linear model in SPM5 (Welcome Department of Cognitive Neurology, London, UK). The fMRI time series were modeled as series of events convolved with a canonical hemodynamic response function. A design matrix was set up to model all task conditions of interest and the six motion parameters. Error trials and trials contaminated with head motion were modeled as two separate predictors that were ignored in the further analyses.

First, a voxel-wise linear regression analysis was performed on the global individual contrast image (all 4 trial types pooled $>$ fixation) to obtain activation patterns correlated with $\mathrm{AUC}_{\mathrm{G}}$ and $\mathrm{AUC}_{\mathrm{I}}$, respectively. Significant associations

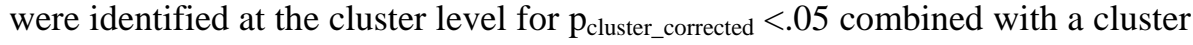
threshold $\geq 10$ voxels. This regression analysis was repeated separately for the young and old adolescents.

Second, we examined differences between responders and non-responders in activation patterns, irrespective of task condition. Individual contrast images were taken to a second level analysis in which subject was treated as a random effect. A whole brain, voxel-wise random-effect ANCOVA was conducted with task difficulty (2 levels) and task response (2 levels) as within-subject variables, group (2 levels) as a between-subject variable, and age as a covariate. We report results of this whole brain analysis using a False Discovery Rate (FDR) error corrected level, which is often applied for group differences in random-effect analyses, combined with a cluster threshold $\geq 10$ voxels. Results of this group comparison were used to define regions of interest (ROI), to compare Percent Signal Change (PSC) between responders and non-responders. The final voxels extracted were identified based on the local maximum of a cluster and a radius of $6 \mathrm{~mm}$. The average PSC of the 20\% strongest responding voxels was calculated for each of the 4 trial types. For each ROI, an ANOVA was conducted with task difficulty (2 levels) and task response (2 levels) as within-subject variables and group (2 levels) as between-subject variable. The assumption of equal error variances across groups (Levene's test) was met for all analyses. Alpha was set at .05 for each ANOVA to give maximal sensitivity.

\section{Results}

Salivary cortisol changes during an fMRI experiment

Salivary cortisol levels changed during the course of the fMRI experiment $\left(\mathrm{F}_{(1}\right.$, 20) $=3.35$; $\mathrm{p}=.03$; Figure 1). Cortisol levels were higher at the start (measure 3) compared with the end (measure 5) of the scan session ( $\mathrm{p}=.01)$. Neither the main effect of age group $\left(\mathrm{F}_{(1,20)}=4.14 ; \mathrm{p}=.06\right)$, nor the inter action term $\left(\mathrm{F}_{(1,20)}=0.53\right.$; 


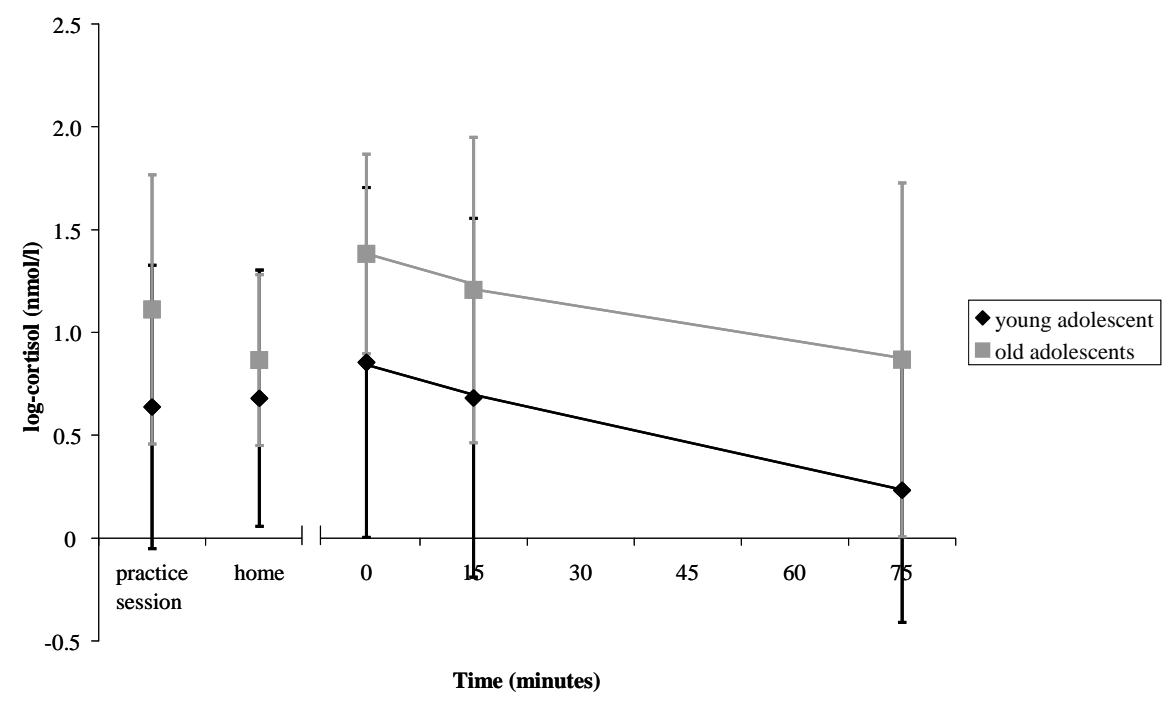

Figure 1: Cortisol changes during the fMRI experiment for each age group. Error bars indicate the standard deviation.

$\mathrm{p}=.72$ ) was significant. Furthermore, age groups did not differ on the cortisol metrics $\mathrm{AUC}_{\mathrm{G}}\left(\mathrm{T}_{(21)}=-1.86 ; \mathrm{p}=.08\right)$ and $\mathrm{AUC}_{\mathrm{I}}\left(\mathrm{T}_{(21)}=-0.60 ; \mathrm{p}=.55\right)$. The nonsignificant trend for higher cortisol values as well as $\mathrm{AUC}_{\mathrm{G}}$ in old compared with young adolescents probably reflects the difference in time of the scan session, which was scheduled earlier in the afternoon for old adolescents. Correspondingly, when we controlled for the time of cortisol measure 3, the trend for an age group difference became weaker for the separate cortisol values ( $\mathrm{p}=.09)$ as well as for $\mathrm{AUC}_{\mathrm{G}}(\mathrm{p}=.17)$. These results suggest that cortisol levels decreased in the time window between the start and end of an fMRI experiment, irrespective of age group.

Salivary cortisol changes in responders versus non-responders

Given the large inter-individual differences, we compared responders and nonresponders on the cortisol levels during the fMRI experiment. The ANOVA showed main effects for group $\left(\mathrm{F}_{(1,20)}=45.29 ; \mathrm{p}<.001\right)$ and cortisol measure $\left(\mathrm{F}_{(1 \text {, }}\right.$ $\left.{ }_{20)}=3.56 ; \mathrm{p}=.03\right)$, as well as an interaction effect $\left(\mathrm{F}_{(1,20)}=4.09 ; \mathrm{p}=.02\right)$. The responders had higher salivary cortisol levels compared with non-responders on all measures except from the home measure (Figure 2). This finding suggests that responders indeed showed elevated cortisol secretion compared with nonresponders during test sessions, but showed comparable cortisol levels in a nonstressful environment. 


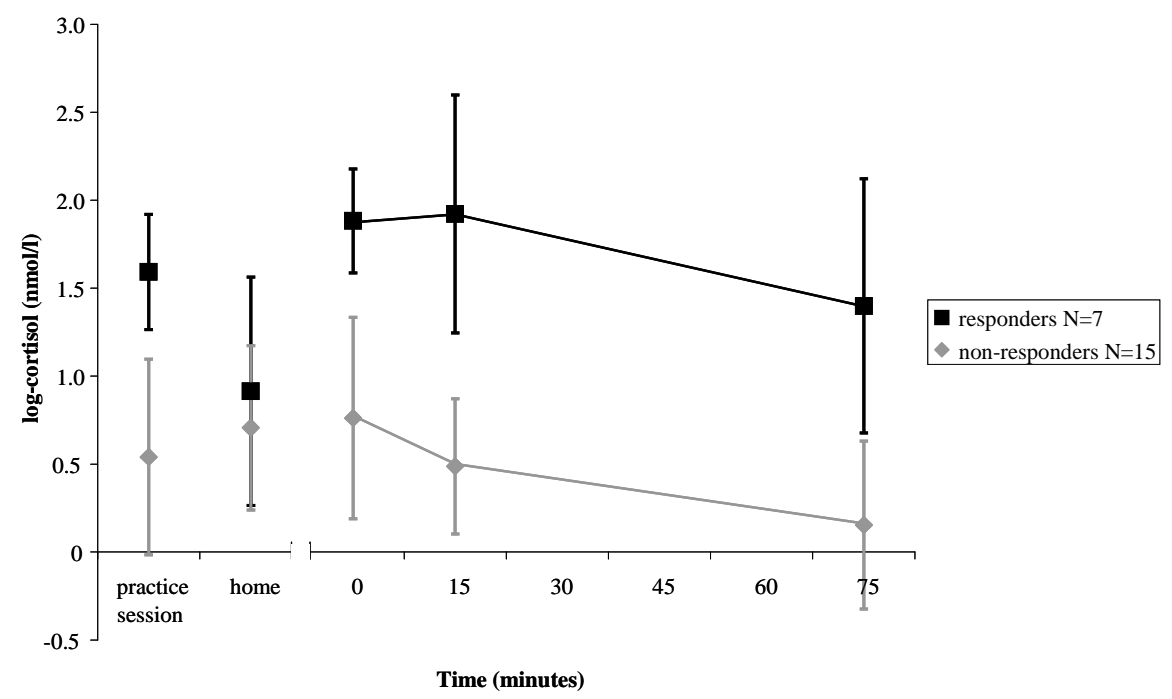

Figure 2: Cortisol changes during the fMRI experiment in responders and non-responders. Error bars indicate the standard deviation.

Relation between cortisol and behavioral performance

There were no significant correlations between salivary cortisol values (individual measures 1 through $5, \mathrm{AUC}_{\mathrm{G}}$ or $\mathrm{AUC}_{\mathrm{I}}$ ) and any of the gambling performance variables. In addition, responders and non-responders did not differ on reaction times, exo PASS errors, negative feedback or total points.

Correlations between cortisol and brain activation

A linear voxel-wise regression analysis was carried out to identify specific brain regions associated with cortisol levels during fMRI scanning. Table 1 lists a total overview of clusters that correlated significantly with one of the AUC values. The $A_{U C}$, indicating total cortisol output, was positively correlated with activation in the right cuneus and negatively correlated with activation in the right lateral inferior parietal cortex. We also calculated correlations between $\mathrm{AUC}_{\mathrm{G}}$ and brain activation in the separate adolescent age groups. In young adolescents, total cortisol output was positively correlated with activation in the left superior parietal lob. In addition, cortisol output correlated negatively with brain activation in several areas, namely two clusters in ventral medial prefrontal cortex and right precentral sulcus. In old adolescents, there were neither positive nor negative significant correlations between $\mathrm{AUC}_{\mathrm{G}}$ and brain activation.

The $\mathrm{AUC}_{\mathrm{I}}$, indicating changes in cortisol relative to the pre-imaging level, was negatively correlated with activation in the left middle temporal gyrus. The correlation analysis in the separate adolescent age groups yielded significant 
Table 1: Results of the correlation analyses between cortisol and brain activation

\begin{tabular}{|c|c|c|c|c|c|c|c|c|c|}
\hline \multicolumn{5}{|c|}{$\mathrm{AUC}_{\mathrm{G}}$} & \multicolumn{5}{|c|}{$\mathbf{A U C}_{\mathbf{I}}$} \\
\hline TAL & size & $\mathrm{F}$ & $\mathrm{p}$ & brain area & TAL & size & $\mathrm{F}$ & $\mathrm{p}$ & brain area \\
\hline \multicolumn{10}{|c|}{ All participants $(\mathrm{N}=22)$} \\
\hline \multicolumn{10}{|c|}{ Positive correlations } \\
\hline $12-7527$ & 17 & 5.28 & .038 & cuneus & \multicolumn{5}{|c|}{ non-significant } \\
\hline \multicolumn{10}{|c|}{ Negative correlations } \\
\hline $45-5136$ & 18 & 5.18 & .030 & lateral IPC & $-48-633$ & 24 & 5.51 & .007 & MTG \\
\hline \multicolumn{10}{|c|}{ Young adolescents $(\mathrm{N}=11)$} \\
\hline \multicolumn{10}{|c|}{ Positive correlations } \\
\hline$-30-5157$ & 10 & 8.48 & .022 & SPL & $-9-36-21$ & 10 & 6.40 & .025 & cerebellum \\
\hline & & & & & $-12-315$ & 12 & 3.72 & .009 & caudate \\
\hline \multicolumn{10}{|c|}{ Negative correlations } \\
\hline$-345-6$ & 10 & 8.33 & .022 & vmPFC & $-33-360$ & 15 & 10.7 & .002 & MFG \\
\hline$-933-3$ & 19 & 7.79 & .001 & vmPFC & $3-6921$ & 23 & 8.49 & .001 & precuneus \\
\hline \multirow[t]{4}{*}{$42-1530$} & 12 & 6.99 & .008 & precentral & 303312 & 12 & 7.88 & .009 & MFG \\
\hline & & & & & $-48-633$ & 22 & 7.68 & .001 & MTG \\
\hline & & & & & $15-345$ & 23 & 7.35 & .001 & cingulate \\
\hline & & & & & 42429 & 10 & 6.10 & .025 & \\
\hline
\end{tabular}

Old adolescents $(\mathrm{N}=\mathbf{1 1})$

No significant results for either positive or negative correlations

Note. This table presents clusters in which brain activation during the gambling task significantly correlated with cortisol, measured as the Area Under the Curve with respect to both ground (AUCG) and pre-imaging level (AUCI). These correlations were calculated for all participants together, as well as for young and old adolescents separately. Associations were considered significant at the cluster level for pcluster-corrected $<.05$ combined with a cluster threshold $\geq 10$ voxels. TAL $=$ Talairach coordinates; size $=$ cluster size indicated by the number of voxels; IPC = Inferior Parietal Cortex; MTG= Middle Temporal Gyrus; SPL= Superior Parietal Lob; vmPFC= ventral medial Prefrontal Cortex; MFG= Middle Frontal Gyrus; IFS= Inferior Frontal Sulcus.

clusters only in young adolescents. A stronger increase in cortisol during the scan session was associated with higher activation in the left cerebellum and in the body of the caudate nucleus. In addition, $\mathrm{AUC}_{\mathrm{I}}$ was negatively correlated with brain activation in several areas in the frontal, cingulate and temporal cortices.

These findings suggest that total cortisol output $\left(\mathrm{AUC}_{\mathrm{G}}\right)$ and cortisol changes over time $\left(\mathrm{AUC}_{\mathrm{I}}\right)$ were related to activation in different areas of the brain. Furthermore, associations between cortisol and the BOLD response were revealed in young adolescents but not in old adolescents.

Brain activation differences between responders and non-responders

The whole brain, voxel-wise analysis revealed two clusters that significantly differed between responders and non-responders for the global task contrast at the FDR corrected level (FDR $<.05 ; \mathrm{F}=22.53$ ), namely in the right inferior parie- 
tal cortex (Talairach coordinates: 30, -54, 48 and 48, -42, 45). These clusters overlapped with task relevant, positive responding areas. In both inferior parietal ROIs the PSC was higher in non-responders compared with responders $\left(F_{(1}\right.$, ${ }_{20)}=11.11 ; \mathrm{p}=.003$ and $\mathrm{F}_{(1,20)}=19.56 ; \mathrm{p}<.001$, respectively).

\section{Discussion}

The present study examined stress-related cortisol reactivity in two adolescent age groups during an fMRI experiment. Pre-imaging salivary cortisol levels were elevated compared with post-imaging levels, suggesting anticipatory stress regarding fMRI scanning, which decreased during the course of the scan session. This pattern applied to all participants, irrespective of age. The reduction in anticipatory anxiety has been previously observed in adults undergoing (f)MRI (Dantendorfer et al., 1997). Although the decrease of cortisol during the scan session might partially reflect the diurnal rhythm of cortisol, this effect is minimized by scanning in the late afternoon when the diurnal fluctuations are relatively small. In addition, the present data showed large individual differences in the total cortisol output during the scan session $\left(\mathrm{AUC}_{\mathrm{G}}\right)$, resulting in a distinction of responders and non-responders as has been previously done by Pruessner et al. (2003, 2008). Responders showed higher salivary cortisol levels compared with non-responders during both practice and imaging sessions, but showed comparable cortisol levels at home. This suggests a general tendency, not specifically related to fMRI, for responders to increase cortisol in situations that are probably experienced as stressful. The enhanced cortisol output in responders was independent of age, suggesting that the psychological distress associated with (f)MRI participation is comparable throughout adolescence.

Higher cortisol output during the scan session in responders compared with non-responders was associated with reduced activation in the right inferior parietal cortex. The right inferior parietal cortex has been described as part of the dorsal attention network (Corbetta and Shulman, 2002; Fox et al., 2006) as well as the task positive network (Dosenbach et al., 2007; Sridharan et al., 2008). This finding might be explained by a disruption of ongoing cognitive activities by the stress related to fMRI scanning, which redirects attentional resources from the gambling task towards stress-related internal thoughts (Seibert and Ellis, 1991). In line with this interpretation, subjects with posttraumatic stress disorder have been characterized by cognitive deficits related to attention and attenuated BOLD responses in attention-related parietal regions during task performance (Pannu Hayes et al., 2009).

Another important finding of the present study is that significant correlations between cortisol and brain activation were found in young but not in old adolescents. This suggests an age-dependent effect of cortisol on the brain, despite the 
fact that age groups were indistinguishable in cortisol reactivity. Young adolescents may be less able to self-regulate the psychological distress associated with imaging, which consequently interferes with task related activation. This explanation is in line with developmental studies showing that cognitive control continues to develop throughout adolescence (e.g., Huizinga et al., 2006; Luna et al., 2004). The two measures of cortisol, i.e., total cortisol output ( $\mathrm{AUC}_{\mathrm{G}}$ ) and cortisol change relative to pre-imaging level $\left(A_{U} C_{I}\right)$, were associated with the BOLD response in different areas of the brain. This finding corresponds to the suggestion of Pruessner et al. (2003) that the areas under the curve with respect to either ground or pre-imaging level were associated with different psychological variables. In young adolescents, a higher cortisol output was associated with a decrease in ventral medial prefrontal activation as well as with increased superior parietal activation. These findings are in line with previous reports of involvement of the medial prefrontal cortex in modulating the stress response (Kern et al., 2008; Pruessner et al., 2008), as well as positive associations between cortisol and parietal activation (Wang et al., 2005; Weerda et al., 2010). Increase of cortisol during the scan session relative to the pre-imaging level was associated with attenuated activation in medial and lateral prefrontal as well as temporal clusters in young adolescents. These areas have been related to psychological stress and cortisol reactivity in previous studies (Kern et al., 2008; Wang et al., 2005). More specifically, the association between cortisol and prefrontal activation is consistent with the high level of cortisol receptors present in this area (Lupien et al., 2007).

In interpreting the present results, the sample size should be taken into consideration. Given large inter-individual variability in both HPA axis reactivity and brain activation patterns, the relatively small sample of 22 participants (of whom 11 young and 11 old adolescents) might have influenced the significance of the results. Nevertheless, this study did reveal some significant associations between cortisol and brain activation. This suggests that the present findings might be a conservative estimation of the potential importance of stress in imaging experiments. Future research should not only include larger sample sizes but also an additional child and adult group to examine developmental effects in stress reactivity to (f)MRI scanning over a broader age range. When considering the possible effect of the imputation of two missing cortisol measures, the present findings remained unchanged when we excluded the two participants with missing values from the analyses.

In summary, the present results provide the first evidence that individual differences in cortisol reactivity to fMRI scanning might have a confounding influence in imaging experiments. Although young and old adolescents both showed anticipatory stress responses that decreased during the scan session, cortisol was associated with the BOLD response only in young adolescents. This finding implies that cortisol may cloud developmental fMRI findings, as the 
effect of cortisol on brain activation is age-dependent. Moreover, a subgroup of participants could be distinguished, irrespective of age, who showed elevated salivary cortisol levels during the fMRI experiment. This cortisol response pattern was associated with attenuated activation in the inferior parietal cortex. Therefore, cortisol reactivity to fMRI scanning should be considered as a confounding factor and should be included in the planning, execution and analyses of fMRI data, especially in studies comparing age groups. 


\section{References}

Corbetta, M., Shulman, G.L., 2002. Control of goal-directed and stimulus-driven attention in the brain. Nat. Rev. Neurosci. 3, 201-215.

Dantendorfer, K., Amering, M., Bankier, A., Helbich, T., Prayer, D., Youssefzadeh, S., Alexandrowicz, R., Imhof, H., Katschnig, H., 1997. A study of the effects of patient anxiety, perceptions, and equipment on motion artifacts in magnetic resonance imaging. Magn. Reson. Imaging 15, 301-306.

Dedovic, K., D'Aguiar, C., Pruessner, J.C., 2009a. What stress does to your brain: a review of neuroimaging studies. Can. J. Psychiatry 54, 17-25.

Dedovic, K., Duchesne, A., Andrews, J., Engert, V., Pruessner, J.C., 2009b. The brain and the stress axis: The neural correlates of cortisol regulation in response to stress. Neuroimage 47, 864-871.

Dedovic, K., Renwick, R., Mahani, N.K., Engert, V., Lupien, S.J., Pruessner, J.C., 2005. The Montreal Imaging Stress Task: using functional imaging to investigate the effects of perceiving and processing psychosocial stress in the human brain. J. Psychiatry Neurosci. 30, 319-325.

de Kloet, E.R., Oitzl, M.S., Joels, M., 1999. Stress and cognition: Are corticosteroids good or bad guys? Trends Neurosci. 22 (10), 422-426.

de Quervain, D.J., Henke, K., Aerni, A., Treyer, V., McGaugh, J.L., Berthold, T., Nitsch, R.M., Buck, A., Roozendaal, B., Hock, C., 2003. Glucocorticoid-induced impairment of declarative memory retrieval is associated with reduced blood flow in the medial temporal lobe. Eur. J. Neurosci. 17 (6), 1296-1302.

de Quervain, D.J., Roozendaal, B., Nitsch, R.M., McGaugh, J.L., Hock, C., 2000. Acute cortisone administration impairs retrieval of long-term declarative memory in humans. Nat. Neurosci. 3 (4), 313-314.

Dickerson, S., Kemeny, M., 2004. Acute stressors and cortisol responses: a theoretical integration and synthesis of laboratory research. Psychol. Bull. 130 (3), 355-391.

Dosenbach, N.U.F, Fair, D.A., Miezin, F.M., Cohen, A.L., Wenger, K.K., Dosenbach, R.A.T., Fox, M.D., Snyder, A.Z., Vincent, J.L., Raichle, M.E., Schlaggar, B.L., Petersen, S.E., 2007. Distinct brain networks for adaptive and stable task control in humans. Proc. Natl. Acad. Sci. USA 104 (26), 11073-11078.

Eatough, E.M., Shirtcliff, E.A., Hanson, J.L., Pollak, E.A., 2009. Hormonal reactivity to MRI scanning in adolescents. Psychoneuroendocrinology 34, 1242-1246.

Fox, M.D., Corbetta, M., Snyder, A.Z., Vincent, J.L., Raichle, M.E., 2006. Spontaneous neuronal activity distinguishes human dorsal and ventral attention systems. Proc. Natl. Acad. Sci. USA 103 (26), 10046-10051.

Friston, K.J., Williams, S., Howard, R., Frackowiak, R.S.J., Turner, R., 1996. Movementrelated effects in fMRI time-series. Magn. Reson. Med. 35 (3), 346-355.

Huizinga, M., Dolan, C.V., van der Molen, M.W., 2006. Age-related changes in executive functions: Developmental trends and a latent variable analysis. Neuropsychologia 44, 2017-2036.

Johnstone, T., Ores Walsh, K.S., Greischar, L.L., Alexander, A.L., Fox, A.S., Davidson, R., Oakes, T.R., 2006. Motion correction and the use of motion covariates in multiplesubject fMRI analysis. Hum. Brain Map. 27, 779-788.

Kern, S., Oakes, T.R., Stone, C.K., McAuliff, E.M., Kirschbaum, C., Davidson, R.J., 2008. Glucose metabolic changes in the prefrontal cortex are associated with HPA axis response to a psychosocial stressor. Psychoneuroendocrinology 33, 517-529. 
Keulers, E.H.H., Stiers, P., Jolles, J., 2010 Developmental changes between ages 13-21 in the extent and magnitude of the BOLD response during decision making. NeuroImage, in press.

Kirschbaum, C., Hellhammer, D., 1994. Salivary cortisol in psychoneuroendocrine research: recent developments and applications. Psychoneuroendocrinology 19, 313-333.

Kirschbaum, C., Wolf, O.T., May, M., Wippich, W., Hellhammer, D.H., 1996. Stress- and treatment-induced elevations of cortisol levels associated with impaired declarative memory in healthy adults. Life Sci. 58 (17), 1475-1483.

Kukolja, J., Thiel, C.M., Wolf, O.T., Fink, G.R., 2008. Increased cortisol levels in cognitively challenged situations are beneficial in young but not older subjects. Psychopharmacology 201, 293-304.

Luna, B., Garver, K.E., Urban, T.A., Lazar, N.A., Sweeney, J.A., 2004. Maturation of cognitive processes from late childhood to adulthood. Child Development, 75 (5), 1357-1372.

Lupien, S.J., Maheu, F., Tu, M., Fiocco, A., Schramek, T.E., 2007. The effects of stress and stress hormones on human cognition: Implications for the field of brain and cognition. Brain Cogn. 65, 209-237.

Lupien, S.J., Wilkinson, C.W., Briere, S., Menard, C., Ng Ying Kin, N. M., Nair, N.P., 2002. The modulatory effects of corticosteroids on cognition: Studies in young human populations. Psychoneuroendocrinology 27 (3), 401-416.

Mayer, A.R., Franco, A.R., Ling, J., Canive, J.M., 2007. Assessment and quantification of head motion in neuropsychiatric functional imaging research as applied to schizophrenia. J. Int. Neuropsych. Soc. 13, 839-845.

Melendez, J., McCrank, E., 1993. Anxiety-related reactions associated with magnetic resonance imaging examinations. JAMA 270, 745-747.

Mennes, M., Wouters, H., van den Bergh, B., Lagae, L., Stiers, P., 2008. ERP correlates of complex human decision making in a gambling paradigm: Detection and resolution of conflict. Psychophysiology 45, 714-720.

Nelson, E.E., Leibenluft, E., McClure, E.B., Pine, D.S., 2005. The social re-orientation of adolescence: A neuroscience perspective on the process and relation to psychopathology. Psychol. Med. 35, 163-174.

Newcomer, J.W., Selke, G., Melson, A.K., Hershey, T., Craft, S., Richards, K., Alderson, A.L., 1999. Decreased memory performance in healthy humans induced by stress-level cortisol treatment. Arch. Gen. Psychiatry 56 (6), 527-533.

Pannu Hayes, J., LaBar, K.S., Petty, C.M., McCarthy, G., Morey, R.A., 2009. Alterations in the neural circuitry for emotion and attention associated with posttraumatic stress symptomatology. Psychiat. Res. Neuroim. 172, 7-15.

Pruessner, J.C., Dedovic, K., Khalili-Mahani, N., Engert, V., Pruessner, M., Buss, C., Renwick, R., Dagher, A., Meaney, M.J., Lupien, S., 2008. Deactivation of the limbic system during acute psychosocial stress: evidence from positron emission tomography and functional magnetic resonance imaging studies. Biol. Psychiatry 63 (2), 234-240.

Pruessner, J.C., Kirschbaum, C., Meinlschmid, G., Hellhammer, D.H., 2003. Two formulas for computation of the area under the curve represent measures of total hormone concentration versus time-dependent change. Psychoneuroendocrinology 28, 916-931.

Seibert, P.S., Ellis, H.C., 1991. Irrelevant thoughts, emotional mood states, and cognitive task performance. Mem. Cognition 19, 507-513.

Sridharan, D., Levitin, D.J., Menon, V., 2008. A critical role for the right fronto-insular cortex in switching between central-executive and default-mode networks. Natl. Acad. Sci. USA 105 (34), 12569-12574.

Stiers, P., Mennes, M., Sunaert, S., 2010. Distributed task coding throughout the multiple demand network of the human frontal-insular cortex, NeuroImage, 52(1), 252-262. 
Tabachnick, B.G., Fidell, L.S., 2001. Using Multivariate Statistics. Allyn and Bacon, Boston, London.

Tessner, K.D., Walker, E.F., Hochman, K., Hamann, S., 2006. Cortisol responses of healthyvolunteers undergoing magnetic resonance imaging. Hum. Brain Map. 27, 889-896.

Wang, J., Korczykowski, M., Rao, H., Fan, Y., Pluta, J., Gur, R.C., McEwen, B.S., Detre, J.A., 2007. Gender difference in neural response to psychological stress. Soc. Cogn. Affect. Neurosci. 2, 227-239.

Wang, J., Rao, H., Wetmore, G.S., Furlan, P.M., Korczykowski, M., Dinges, D.F., Detre, J.A., 2005. Perfusion functional MRI reveals cerebral blood flow pattern under psychological stress. Natl. Acad. Sci. USA 102, 17804-17809.

Weerda, R., Muehlhan, M., Wolf, O.T., Thiel, C.M., 2010. Effects of acute psychosocial stress on working memory related brain activity in men. Hum. Brain Map, in press.

Yoo, S., Choi, B., Juh, R., Pae, C., Lee, C., 2005. Head motion analysis during cognitive fMRI examination: Application in patients with schizophrenia. Neurosci. Res. 53, 8490. 


\section{Chapter}

Sex differences in brain activation are related to motivational context rather than to the nature of decision making 


\begin{abstract}
The present study examined sex-related differences in brain activation during decision making. Previous studies revealed discrepant sex effects in neurocognitive functions, which might be caused by using different task paradigms. Therefore, we used two decision making tasks that differed along two dimensions: motivational context, and exogenous versus endogenous decision making. A homogenous sample of young adults aged 20-21 years performed a gonogo task (low motivational context, exogenous decision making) as well as a gambling task (high motivational context) with both exogenous and endogenous decision making trials. Men reported a higher sensation seeking tendency on a self-report questionnaire than women, although behavioral performance on both decision making tasks was comparable between male and female young adults. Nevertheless, we did reveal sex effects in brain activation during both exogenous and endogenous gambling trials. There were no sex effects during the gonogo task. In two task-related areas, anterior cingulate cortex and lingual gyrus, men showed a higher BOLD response than women. In addition, some task-induced deactivations were modulated by sex. Clusters in inferior frontal sulcus and precuneus were deactivated only by men, whereas a posterior cingulate cluster was deactivated only by women. These sex-related functional changes underlying gambling performance were not mediated by the higher sensation seeking in men, except for the anterior cingulate cortex. The present results suggest that sex differences in brain activation during decision making are related to the motivational context, rather than to the nature of decision making.
\end{abstract}




\section{Introduction}

It has long been recognized that men and women differ in behavior, cognitive profile and risk for psychopathology. These sex differences become more pronounced during adolescence, following hormonal changes related to puberty (Giedd et al., 2006; Sisk \& Zehr, 2005). The behavioral and cognitive differences have increasingly been related to sex-related distinctions in brain structure and functioning. Sex is indeed known to influence the rate of structural brain development, as girls reach peak grey matter thickness 1-2 years earlier than boys (Blanton et al., 2004; Giedd et al., 2006). In addition, differences between male and female adult brains have been found. Men are characterized by larger overall brain volumes as well as higher white matter and cerebrospinal fluid percentages (Leonard et al., 2008; Luders et al., 2005), whereas in women larger regional grey matter volumes in for example caudate, frontal, temporal and parietal cortices as well as thicker cortices have been shown (Good et al., 2001; Luders et al., 2009; Sowell et al., 2007).

Traditionally, sex differences in adults have been mostly examined for verbal and spatial abilities. Women outperform men at verbal tasks such as verbal memory and word fluency, whereas men are superior on spatial tasks, and especially on mental rotation (Kimura, 1996; Linn \& Petersen, 1985; Voyer et al., 1995). However, functional MRI studies examining sex differences in verbal and spatial processing have revealed contradictory results, describing both the existence (Kucian et al., 2005; Shaywitz et al., 1995; Weiss et al., 2003) and the absence (Schlosser et al., 1998; Unterrainer et al., 2000) of brain activation differences between men and women. A similar contradiction is observed with respect to sex-related differences in brain activation of adults performing cognitive control tasks, such as working memory (Bell et al., 2006; Haut \& Barch, 2006; Speck et al., 2000) and response inhibition (Halari \& Kumari, 2005; Li et al., 2006) tasks.

Discrepancies between fMRI studies may partially be caused by the confounding influence of sample characteristics, such as age. For example, some studies used a broad age range (20-40 years; Kucian et al., 2005; Li et al., 2006), resulting in a heterogeneous group. Structural brain as well as socio-emotional changes are not yet fully matured in 20 year-olds (Nelson et al., 2005; Sowell et al., 1999). In addition, evidence suggests that neurocognitive performance already differs between ages 20 and 40 (Van der Elst et al., 2006). The use of different task paradigms requiring different cognitive functions may also contribute to heterogeneity in sex-related brain activation effects. Most of the studies used simple stimulus-response tasks, in which the decision to respond in a particular way is prescribed by external signals. These relative easy tasks can be 
performed with a minimum of cognitive effort and might lack the challenge to consistently trigger sex differences in brain activation. Manipulating task aspects that make a task more challenging, such as difficulty level, motivational context or emotional content might differently affect sex differences in brain activation. One such factor that has consistently triggered sex differences is the presence of a motivational context, such as implemented in gambling and decision making tasks in which points or other rewards can be obtained (e.g. Lee et al., 2009; Reavis \& Overman, 2001).

Male adults are superior to female adults in behavioral performance on gambling and decision making tasks, such as the Iowa Gambling Task (Bolla et al., 2004; Reavis \& Overman, 2001). In addition, men have shown a higher propensity for impulsive, risk taking and sensation seeking behavior compared with women (Byrnes et al., 1999; Rosenblitt et al., 2001). This suggests that there are sex-related differences in the neural mechanisms that control motivational decision making. This was confirmed in two neuroimaging studies in adults aged 2040 (Bolla et al., 2004; Lee et al., 2009), both of which focused on anatomically determined regions of interests (ROI) implicated in decision making: the insula, and the orbital and dorsolateral prefrontal cortices (Manes et al., 2002; O’Doherty et al., 2001; Paulus, 2005). Women showed stronger activation than men in dorsolateral prefrontal cortex and insula, whereas both stronger and weaker activation was reported in the orbital frontal cortex of women compared with men (Bolla et al., 2004; Lee et al., 2009). These sex effects were subtle, however, as the results did not survive multiple comparisons correction when examined in a whole brain analysis (Bennett et al., 2009; Bolla et al., 2004; Lee et al., 2009).

Given that increased risk taking as well as poor decision making tendencies are characteristic of adolescence and even of young adulthood (Dahl, 2004; Gardner \& Steinberg, 2005; Paus et al., 2008; Steinberg, 2008a), this is an interesting age window to study sex effects. A higher sensation seeking tendency in male compared with female adolescents has been revealed, although this sex effect was the same for different age ranges within adolescence and young adulthood (Steinberg et al., 2008b). Conversely, meta analysis results of Byrnes and colleagues (1999) did show that this sex difference in risk taking became smaller over the age period of adolescence and young adulthood. This latter finding is in line with sex-specific structural developmental trajectories throughout adolescence and adulthood, with women maturing faster than men (Giedd et al., 1999, 2006). No functional neuroimaging study, however, investigated sex differences in the neural mechanisms engaged in decision making in the age window of adolescence and young adulthood.

In the present study, we examined sex differences in brain activation during decision making in a homogenous sample of young adults aged 20-21. To establish whether motivational context is indeed an important factor in revealing sex 
differences, we used two tasks that differed in motivational context. First, a simple gonogo task was used, in which the motivational context was low. The decision to respond or not was exogenously controlled in this task. Second, participants performed a challenging gambling task in a high motivational context, as they could cumulate points over trials. Next to motivational context, gambling tasks are characterized by endogenous control of behavior as opposed to the exogenous controlled gonogo task, in which the decision to respond in a particular way is guided from within oneself (Mennes et al., 2009; Miller, 2000; Brass \& von Cramon, 2004). Therefore, in the present gambling task the probability of winning and points that could be won were manipulated in order to distinguish between exogenous versus endogenous controlled decision trials, based on whether or not the task stimuli suggested the appropriate response. We hypothesized differential activation patterns between male and female young adults during decision making, only in a high (gambling task) as opposed to a low (gonogo task) motivational context. Furthermore, we expected the sexrelated functional differences to be more pronounced during the endogenous as opposed to exogenous gambling trials. Sex-related differences in brain activation were examined at the whole brain level instead of predetermined ROI analyses in order to explore sex effects unrestrictedly. As a last research question we examined sex differences in sensation seeking behavior and its association with brain activation during execution of the decision making tasks.

\section{Materials and methods}

\section{Participants and procedure}

University students (20/21 year-olds) were recruited through advertisements. All participants had normal or corrected-to-normal vision, were free from psychiatric and neurological abnormalities, did not use medication that could influence cognitive functioning and were screened for MRI contra-indications. Only women using monophasic combination oral contraceptives were included to control for the possible confounding influence of hormone levels on cognition (Amin et al., 2006; Fernandez et al., 2003). Women were scheduled on pilltaking days as the dosages of estrogen and progesterone were the same in each pill and therefore comparable between the female subjects. Written informed consent was obtained from all participants. Participants received a fee of 25 euros. The study was approved by the ethical committee of the Faculty Psychology and Neuroscience of Maastricht University. From the total of 35 participants included in the study, 1 male participant had to be excluded due to technical problems, 1 male participant due to poor behavioral performance on the gonogo task (error rate $>30 \%$ ) and 2 male participants because of too few trials in one 
gambling condition (endogenous GAMBLE). This resulted in a final sample of 31 right-handed participants divided into a male $(\mathrm{N}=15$, mean age $=21.0$, $\mathrm{SD}=0.6)$ and a female $(\mathrm{N}=16$, mean age $=20.8, \mathrm{SD}=0.6)$ group.

Participants attended a training session on average one week before the scan session. The training session served to familiarize participants with the scanning environment and procedure, and to practice the experimental tasks using a mock scanner. Furthermore, two neuropsychological tests were administered to obtain estimates of intelligence. Men and women did neither differ on an estimate of verbal intelligence $\left(\mathrm{M}=108.2, \mathrm{SD}=8.0, \mathrm{~T}_{(30)}=1.18 ; \mathrm{p}=.25\right)$ as assessed with the Peabody Picture Vocabulary Test-III-NL (Dunn \& Dunn, 2005) nor on an estimate of nonverbal intelligence $\left(\mathrm{M}=120.4, \mathrm{SD}=6.6, \mathrm{~T}_{(30)}=0.22 ; \mathrm{p}=.83\right)$ as assessed with the Raven Standard Progressive Matrices (Raven et al., 1998). The Adult Self Report (Achenbach \& Rescorla, 2003) was administered to screen for behavioral problems. All participants had scores on the total problem scale of these questionnaires within $1 \mathrm{SD}$ of the mean of a normative standardized sample. Furthermore, men and women did not differ on the total problem scale of the self report questionnaire $\left(\mathrm{T}_{(30)}=-0.24 ; \mathrm{p}=.81\right)$.

\section{Instruments}

\section{Gonogo task}

Exogenous decision making in a low motivational context was assessed with a gonogo paradigm. The gonogo task consisted of three letter stimuli (" $\mathrm{X}$ ", " $\mathrm{B}$ " and " $\mathrm{A}$ ") that were randomly presented using a fast event-related design. Participants were instructed to press the button every time a letter appeared on the screen, but to withhold their response when an "A" appeared. The letter stimuli corresponded to three conditions: GO ("X”), GOoddball ("B”) and NOGO ("A"). In $60 \%$ of all trials, the letter " $\mathrm{X}$ " was shown, whereas the letters " $A$ " and " $B$ " both appeared in $20 \%$ of the trials. The higher percentage of GO stimuli allowed for the build up of a prepotent tendency to respond. The GOoddball stimuli required a response comparable to the GO trials, but occurred as often as the NOGO trials to control for the effect of low-frequent occurrence (Chikazoe et al., 2009; Hampshire et al., 2010). Letters were presented visually at the centre of the screen, with a duration of 200 ms. During inter stimulus intervals a fixation cross was shown and jittering was applied to these intervals. The inter stimulus interval varied between 1000 and 2000 ms, with an average duration of 1500 ms. In addition, null-events with the same duration and inter stimulus interval as the letter trials were included (33\% of all trials) in which the fixation cross remained visible, to allow for better separation of the BOLD responses. The task was presented in two runs, each consisting of 260 trials, of which 175 were letters. Duration of each run was 7.48 minutes. 


\section{Gambling task}

An event-related gambling task was used to assess decision making in a high motivational context. Participants could decide to either gamble or pass in order to earn as many points as possible (Mennes et al., 2008; Stiers et al., 2010). In each trial a horizontal bar divided into two colored parts was presented (range from $5 \%-95 \%$ to $50 \%-50 \%$ ), indicating the probability of an imaginary token being hidden underneath. Participants could guess under which part a token was hidden, by pressing the corresponding left or right button. Depending on the correctness of their choice, points could be won or lost. The points that could be won were indicated above the bar (range 10-100). The points that could be lost were presented below the bar (range 0-100), with the most ambiguous proportions (50\%-50\%) coupled with the highest losses (80-100 points). Participants could also choose to pass by withholding their response, which resulted consequently in receiving a small 20 points reward. All participants started with 100 points. Feedback about the trial and an update of their total score was provided in only $67 \%$ of the trials, in order to disentangle the effects related to feedback processing from those related to the decision making process.

The behavioral results from a previous study with this task (Mennes et al., 2008) were used to delineate four trial groups along two dimensions: 1) task difficulty: exogenous versus endogenous trials depending on whether stimulus characteristics suggested the appropriate choice or not, and 2) task response: gambling versus passing trials depending on whether a button was pressed or not. Trials characterized by a high probability of winning (80-95\%) and a high gain ( $\geq 30$ points) in which participants should always gamble were exogenous gambling trials (exo GAMBLE). Trials characterized by a gain of 20 or less points in which participants should always pass were exogenous passing trials (exo PASS). Gambling in these trials is disadvantageous as participants received a 20 points reward anyway when passing. Decisions on exogenous GAMBLE and PASS trials were relatively easy and determined by stimulus characteristics such as the probability of winning and points that could be won. These trials are considered to measure exogenous decision making. All other trials characterized by an uncertain probability of winning (50-75\%) and a gain of 30 points or more were considered endogenous trials. Endogenous trials were more difficult as it was not immediately clear based on the stimulus characteristics which response was the most favorable. A final decision in such trials was guided by the participant and required interpretation of different kinds of information such as previous experiences, gain, gain/loss ratio, total score and internal motivational states. Based on whether the participant decided to gamble or pass, these endogenous trials were labeled respectively endo GAMBLE or endo PASS. The task comprised three runs of 7.3 minutes and 50 trials each. The bar was presented for 3.5 seconds and was, in 2/3 of the trials, followed $0.2-2.0$ seconds later by feedback 
for 1.7 seconds. The interval between the end of the feedback and the start of a new trial varied between 1.0-4.0 seconds.

\section{Brief Sensation Seeking Scale}

The Brief Sensation Seeking Scale (BSSC; Hoyle et al., 2002, the originally English version was translated into Dutch) was used to measure sensation seeking. This self-report questionnaire was created by adapting items from the Form V of the Sensation Seeking Scale (SSS-V; Zuckerman et al., 1978). The BSSC consists of 8 items, representing the original four dimensions of sensation seeking, namely thrill and adventure seeking, experience seeking, disinhibition, and boredom susceptibility. Responses are indicated on a five-point scale labeled, strongly disagree, disagree, neither disagree nor agree, agree and strongly agree. Total sum scores range from 8 to 40, with higher scores indicative of increased sensation seeking. Good reliability and validity data of this scale have been reported (Hoyle et al., 2002).

\section{Behavioral analyses}

The statistical package SPSS 16.0 was used for the analyses. Reaction times on respectively the gonogo and gambling task were analyzed with two analyses of variance (ANOVA) with sex (2 levels) as between-subject variable and trial type (2 levels) as within-subject variable. The reaction time data did not violate the parametric assumption of ANOVA. The ratio endogenous GAMBLE/ PASS trials on the gambling task, indicative of a tendency to gamble, was analyzed using an independent samples T-test with sex (2 levels) as between-subject variable. Gonogo dependent variables, viz number of false alarms (i.e. failure to withhold response on NOGO trials) and misses (i.e. failure to react on GO and GOoddball trials) as well as gambling dependent variables, viz total number of points, number of errors on exogenous PASS trials (i.e. failure to withhold response by a gain of 10 or 20 points) and negative feedback were analysed with a non-parametric Mann-Whitney test due to non-normality. Sex differences in Brief Sensation Seeking Scale sum scores were analyzed using an independent samples T-test with sex (2 levels) as between-subject variable.

\section{Functional Magnetic Resonance Imaging data}

\section{Image acquisition}

A Siemens MAGNETOM Allegra 3T MRI head-only scanner was employed. Head motion was constrained by the use of foam padding. A total number of 32 axial slices covering the whole brain including the cerebellum were imaged by using a T2*-weighted gradient echo planner pulse sequence $(\mathrm{TR}=2000 \mathrm{~ms}$, TE $=30 \mathrm{~ms}, \mathrm{FA}=90, \mathrm{FOV}=224$, slice thickness $=4 \mathrm{~mm}$, matrix size $=64 \times 64$, flip angle $=90^{\circ}$ ). Voxel size was $3.5 \times 3.5 \times 4 \mathrm{~mm}$. A T1-weighted anatomical 
scan was acquired to aid with spatial normalization ( $\mathrm{TR}=2250 \mathrm{msec}$, $\mathrm{TE}=2.6$ msec, flip angle $=9^{\circ}, \mathrm{FOV}=256 \mathrm{~mm}$, slice thickness $=1 \mathrm{~mm}$, matrix size $=256$ $\mathrm{x} 256$, number of slices $=192$ ). Voxel size was 1 x 1 x $1 \mathrm{~mm}$. Slice scanning order was ascending interleaved.

\section{Preprocessing}

Data were preprocessed using BrainVoyager QX, version 1.9 (Brain Innovation, Maastricht, The Netherlands). Images were corrected for slice scan time differences, followed by rigid body motion correction, high-pass temporal filtering, and spatial data smoothing using a Gaussian kernel with a $4 \mathrm{~mm}$ full width at half maximum. Functional data were coregistered with the anatomical volume using rigid body transformation and manual adjustments to obtain optimal fit by visual inspection if necessary. Subsequently, both functional and structural volumes were transformed into standard stereotaxic space using Talairach normalization.

In order to control for the confounding effect of head motion we applied 2 corrections to our data (Friston et al., 1996; Johnstone et al., 2006). Firstly, head motion within one scan may distort the measured fMRI signal by spatial misallocation. Therefore, we identified scans during which head motion exceeded a particular threshold, viz absolute motion difference between 2 successive scans in $\mathrm{z}$ direction greater than $0.4 \mathrm{~mm}\left(1 / 10^{\text {th }}\right.$ of the voxel size), and rotation around $\mathrm{x}$ dimension greater than 0.26 degrees (angle corresponding to $0.4 \mathrm{~mm} \mathrm{z}$ displacement of frontopolar voxels, assuming the rotation point in middle of brain is $88 \mathrm{~mm}$ from the anterior end of the brain frontal pole (Talairach \& Tournoux, 1988)). Task trials (taking into account the hemodynamic response function delay) cause signal intensity changes with peak BOLD values 5 to 6 seconds after their occurrence. These signal changes may overlap in time with head motion a few scans later. Consequently, these signal changes are contaminated with within-scan head motion. Therefore, trials in a preceding time window of 1 to 8 seconds of the identified scan were modeled as an additional event of no interest. Male and female young adults did not differ on the two most affected motion parameters for the remaining scans (Mayer et al., 2007; Yoo et al., 2005), viz translation in the $\mathrm{z}$ direction (gonogo task $\mathrm{F}_{(1,29)}=0.81$; $\mathrm{p}=.38$; gambling task $\mathrm{F}_{(1,29)}=0.39 ; \mathrm{p}=.54$ ) and rotation in the $\mathrm{x}$ direction (gonogo task $\mathrm{F}_{(1 \text {, }}$ 29) $=0.27$; $\mathrm{p}=.61$; gambling task $\left.\mathrm{F}_{(1,29)}=0.00 ; \mathrm{p}=1.00\right)$. Secondly, head motion between successive scans causes signal intensity changes due to inhomogeneities in the magnetic field which are unrelated to the task (Friston et al., 1996). Therefore, we modeled this source of signal variance by including the 6 motion parameters as covariates of no interest in our general linear model (Friston et al., 1996; Johnstone et al., 2006). 


\section{Imaging analyses}

Statistical analyses were performed on individual participants data by using the general linear model in SPM5 (Welcome Department of Cognitive Neurology, London, UK). The fMRI time series were modeled as series of events convolved with a canonical hemodynamic response function. A design matrix was set up to model all task conditions of interest (gonogo task: GO, GOoddball and NOGO trials; gambling task: exo GAMBLE, exo PASS, endo GAMBLE, endo PASS trials, neutral, positive and negative feedback) and the 6 motion parameters. Error trials and trials contaminated with head motion were modeled as two separate predictors that were ignored in the further analyses.

Individual percent signal change maps were created for each condition of interest, based on the estimated weights for each of the conditions, and used as data for a second level analysis in which subject was treated as a random effect. First, we identified brain areas underlying the execution of both the gonogo and gambling task, ignoring sex, in a whole brain voxel-wise general linear model. For the analysis of the gonogo task, an ANOVA with task condition (3 levels) as within-subject variable and sex (2 levels) as between-subject variable was performed. For analyzing the gambling task data, an ANOVA with task difficulty (2 levels) and task response (2 levels) as within-subject variables and sex (2 levels) as between-subject variable was conducted. Task (de)activations were identified for each ANOVA in a contrast that pooled over task conditions and sex, at the Family Wise Error correction level (FWE<.05) combined with a cluster threshold $\geq 10$ voxels.

Second, we examined sex differences in the pooled task contrast, for each task separately. We reported results of this group comparison at the False Discovery Rate error correction level (FDR $<.05)$, which is a less stringent whole brain correction for multiple comparisons (Genovese et al., 2002).

We performed region of interest (ROI) analyses to further investigate sexrelated differences in brain activation. The ANOVA results for both tasks that significantly differed between male and female young adults were used to define ROIs. The final voxels extracted were identified based on the local maximum of a significant cluster revealed by the ANOVA, and a radius of $6 \mathrm{~mm}$. For each ROI, the average percent signal change (PSC) was extracted for each of the task conditions. Only the $20 \%$ voxels with the highest PSC were included to make the amplitude assessments maximally independent of the extent of activation. For each gonogo ROI, an ANOVA was conducted with task condition (3 levels) as within-subject variable and sex (2 levels) as between-subject variable. For each gambling ROI, an ANOVA was performed with task difficulty (2 levels) and task response (2 levels) as within-subject variables and sex (2 levels) as between-subject variable. The assumption of equal error variances across groups (Levene's test) was met for all analyses. Alpha was set at .05 for each ANOVA to give maximal sensitivity. If statistically significant effects were found, post 
hoc comparisons were performed. In addition, associations between sensation seeking and PSC during performance of the decision making tasks were explored in each ROI that was affected by sex.

\section{Results}

Behavioral data

Gonogo task

There was neither a sex difference in reaction time $\left(\mathrm{F}_{(1,29)}=2.43\right.$; $\left.\mathrm{p}=.13\right)$, nor any sex difference in the number of misses ( $\mathrm{GO} \chi_{(30)}^{2}=-0.79, \mathrm{p}=.43$; GOoddball $\left.\chi_{(30)}^{2}=-0.57, \mathrm{p}=.57\right)$ and false alarms $\left(\chi_{(30)}^{2}=-1.61 ; \mathrm{p}=.11\right)$. The ANOVA did show a main effect of trial type independent of $\operatorname{sex}\left(\mathrm{F}_{(1,29)}=7.53\right.$; $\left.\mathrm{p}=.01\right)$, with longer reaction times on GOoddball compared with GO trials.

\section{Gambling task}

One sex difference was present for the gambling task, namely in the number of errors on exogenous PASS trials $\left(\chi_{(30)}^{2}=-2.11 ; p=.04\right)$. Women failed more often than men to withhold their response in trials with a small gain of 10 or 20 points. No further sex-related differences were revealed, neither in reaction times $\left(F_{(1}\right.$, ${ }_{29)}=0.16 ; \mathrm{p}=.69$ ), nor in risk-taking behavior measured with the ratio endogenous GAMBLE/ PASS trials $\left(T_{(30)}=-0.12 ; p=.90\right)$, number of negative feedback $\left(\chi_{(30)}^{2}=-0.60 ; p=.55\right)$ and total number of points $\left(\chi_{(30)}^{2}=-0.93 ; p=.35\right)$. With regard to the task conditions independent of sex, the ANOVA on the reaction time data showed a main effect of trial type, with slower reaction times on endogenous compared with exogenous GAMBLE trials $\left(\mathrm{F}_{(1,29)}=218.50 ; \mathrm{p}<.001\right)$.

These results suggest that in general task performance on decision making tasks was comparable between men and women.

\section{Sensation seeking}

Men scored higher than women on the sum score of the Brief Sensation Seeking Scale $\left(\mathrm{T}_{(29)}=3.56 ; \mathrm{p}=.001\right)$. Details on sum scores on the sensation seeking questionnaire for men and women are shown in Figure 1.

\section{Task activation}

Both gonogo and gambling tasks (de)activated similar brain areas, although (de)activations during the gambling task were more extensive and included additional clusters. This is illustrated in Figure 2 by drawing both positive and negative pooled task effects within each task over one another. In both tasks, positive brain activations were found bilaterally in dorsal part of anterior cingulate cor- 


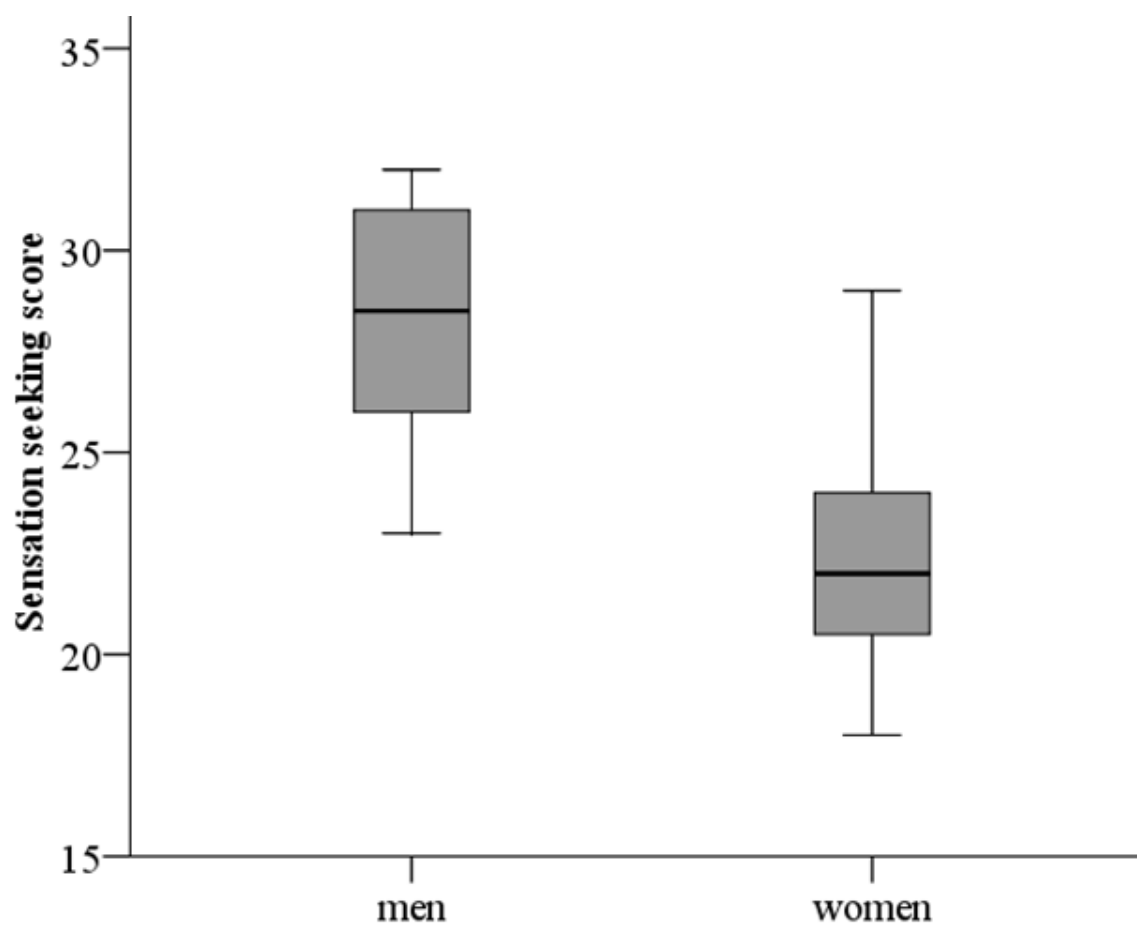

Figure 1: Box plots for the sum score on the Brief Sensation Seeking Scale, showing the minimum, first quartile, median, third quartile, and maximum scores

tex, putamen, anterior insula, occipital areas, left thalamus and right intraparietal sulcus. During the gonogo task left posterior insula was additionally activated. During performance of the gambling task some unique activations were revealed as well, namely bilaterally in the inferior frontal junction, inferior frontal sulcus, frontal eye fields, and parietal areas. These activated brain areas coincide with core functional networks involved in cognitive task execution (e.g. Dosenbach et al., 2007; Duncan \& Owen, 2000; Stiers et al., 2010). Clusters in the ventral medial prefrontal cortex, posterior cingulate cortex, temporal gyri and left parahippocampal gyrus were deactivated during performance of both tasks. In addition, during the gambling task the medial prefrontal cortex, lateral inferior parietal cortex and superior frontal sulcus were deactivated as well. These areas among others have been described as part of the default mode network (e.g. Dosenbach et al., 2007; Greicius et al., 2003; Stiers et al., 2010).

These findings confirm that both tasks were effective in modulating the BOLD signal in functional brain networks, although these network areas were more engaged during the challenging gambling task compared with the gonogo task. 


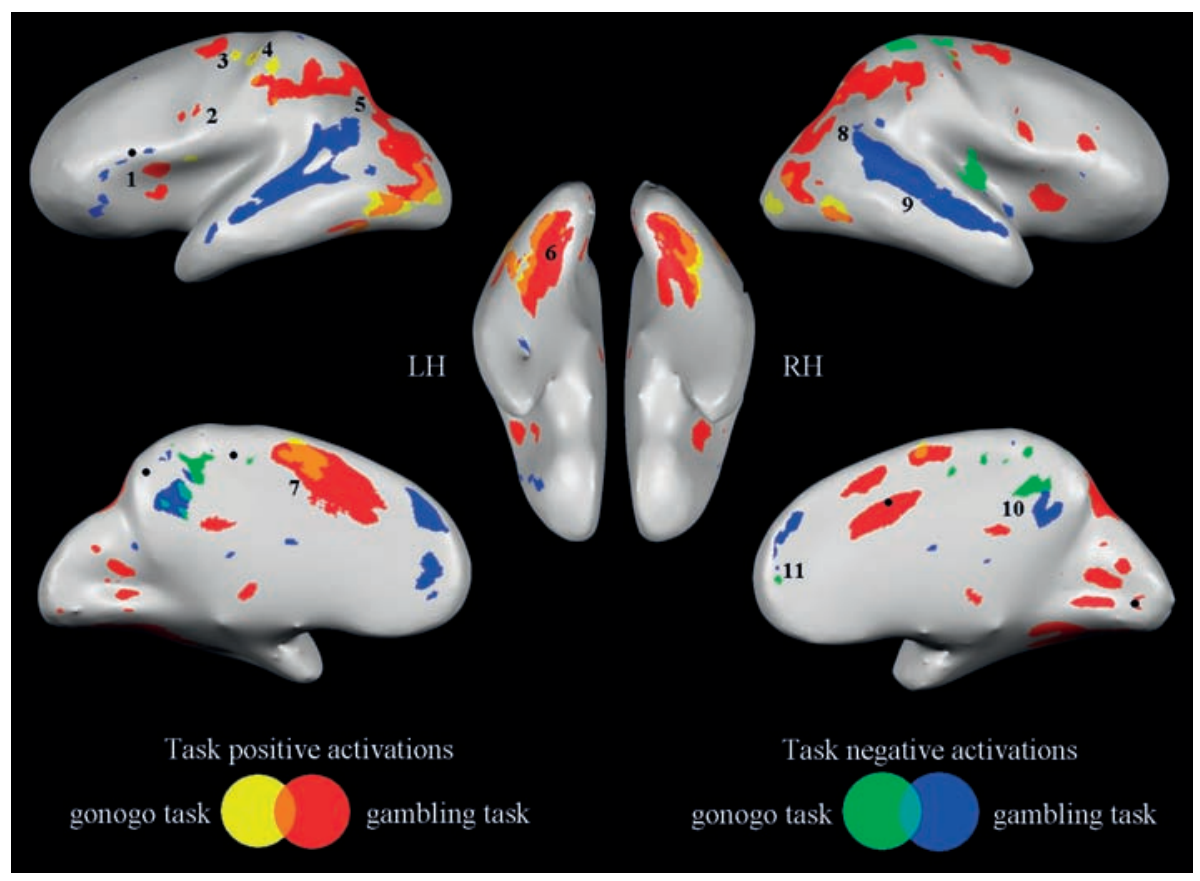

Figure 2: Functional activation patterns during gonogo and gambling task execution, and sexrelated clusters. The functional activation pattern for the gonogo task ( $\mathrm{FWE}<.05$; $\mathrm{T}=4.99)$ is presented in yellow (positive) and green (negative), and for the gambling task ( $\mathrm{FWE}<.05$; $\mathrm{T}=4.89$ ) in red (positive) and blue (negative). Both tasks activate similar brain areas shown by the overlapping clusters, although activations during the gambling task were more extensive and included additional clusters: $1=$ anterior insula, $2=$ inferior frontal junction, $3=$ frontal eye fields, $4=$ postcentral sulcus, $5=$ intraparietal sulcus, $6=$ occipital areas, $7=$ dorsal part of anterior cingulate cortex, $8=$ lateral inferior parietal cortex, $9=$ superior temporal gyrus, $10=$ posterior cingulate cortex, $11=$ ventral medial prefrontal cortex. The five clusters that differed between male and female young adults as revealed by the whole brain comparison during the gambling task are presented as small black circles.

\section{Sex differences in brain activation}

\section{Gonogo task}

The whole brain, voxel-wise analysis revealed no clusters that significantly differed between men and women during performance of the gonogo task at the FDR error corrected level. Repeating the whole brain group comparison for the separate task conditions did also not yield any sex differences.

\section{Gambling task}

The whole brain, voxel-wise analysis revealed five clusters that significantly differed between men and women during the gambling performance at the FDR error corrected level (FDR<.05; F=19.07), namely in the anterior cingulate cortex (Talairach coordinates: 9, 12, 39), lingual gyrus (Talairach coordinates: 15, 
-84, 0), inferior frontal sulcus (Talairach coordinates: $-45,18,24)$, precuneus (Talairach coordinates: $-3,-63,48$ ), and posterior cingulate cortex (Talairach coordinates: $-9,-30,54)$. These clusters are visually presented in Figure 2, relative to task-related (de)activations. We also looked for sex effects in the four specific gambling conditions, as well as the exogenous and endogenous trials. The results for all these conditions largely coincide with the results described above for the global taskcontrast. In line with this finding, there were no significant clusters for interaction effects of sex with respectively the exogenousendogenous and gambling-passing dimensions of the gambling task.

We can conclude therefore that sex differences in brain activation do exist during a challenging gambling task in which points can be won, but such effects were not present -or at least did not reach statistical significance- during an exogenous controlled gonogo task in a low motivational context. Since the sex effects were present in the exogenous as well as the endogenous conditions of the gambling task, they are specific to the motivational task set up in general, rather than to the nature of decision making.

\section{ROI analyses}

We created $6 \mathrm{~mm}$ spherical ROIs centered on the peak activity voxel in regions revealed by the ANOVA that significantly differed between male and female young adults. Since only for the gambling task significant sex effects were found, these 5 ROIs were used here.

The sex-related clusters in anterior cingulate cortex and lingual gyrus overlapped with positive responding areas during gambling execution (Figure 2). In both clusters, men showed higher PSC during all gambling conditions compared with women (respectively $\mathrm{F}_{(1,29)}=8.42 ; \mathrm{p}=.007$ and $\mathrm{F}_{(1,29)}=7.96 ; \mathrm{p}=.008$; Figure $3 \mathrm{~A})$. The remaining three clusters did not overlap with task related areas during performance of the gambling task (Figure 2). Both the inferior frontal sulcus and precuneus were deactivated by men, whereas women did not (de)active these clusters (respectively $\mathrm{F}_{(1,29)}=9.18 ; \mathrm{p}=.005$ and $\mathrm{F}_{(1,29)}=19.70 ; \mathrm{p}<.001$; Figure 3A). Again, these sex differences were independent of gambling condition. In addition, the posterior cingulate cluster was deactivated during the gambling task in women, but not (de)activated in men $\left(F_{(1,29)}=15.07 ; p=.001\right.$; Figure 3A).

We performed these ROI analyses as well for activation during the gonogo task, to explore whether a comparable sex trend was found. In none of the regions was a significant effect found, neither a main effect of sex, nor an interaction of sex with gonogo condition (Figure 3B). There was, however, a trend of higher PSC in the anterior cingulate cortex of men compared with women $\left(\mathrm{F}_{(1}\right.$, 29 ) $=3,29$; $\mathrm{p}=.081$; Figure $3 \mathrm{~B}$ ), which was found as well during execution of the gambling task. 
A Gambling task

right anterior cingulate cortex (9 12 39)

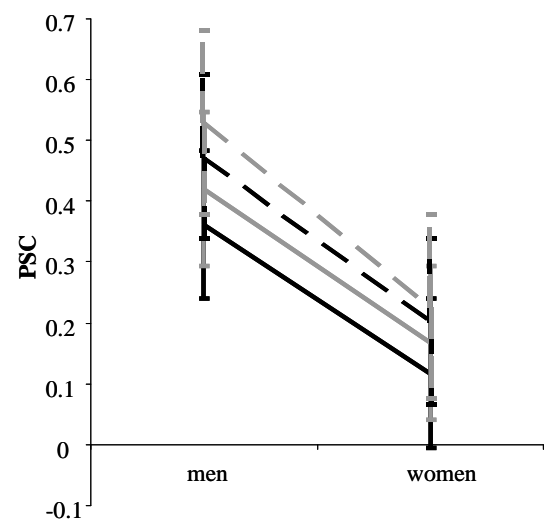

right lingual gyrus (15-84 0)

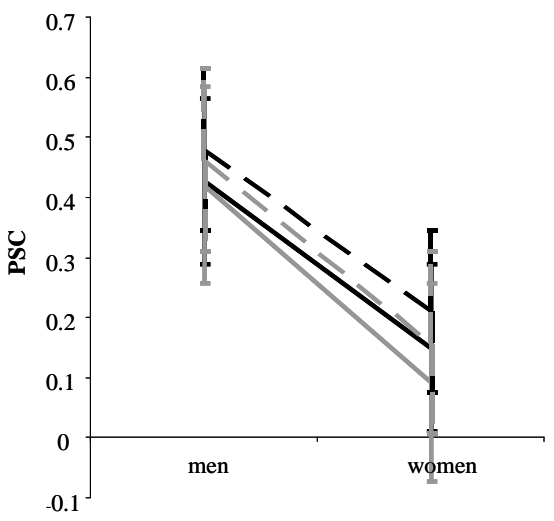

left inferior frontal sulcus (-45 18 24)

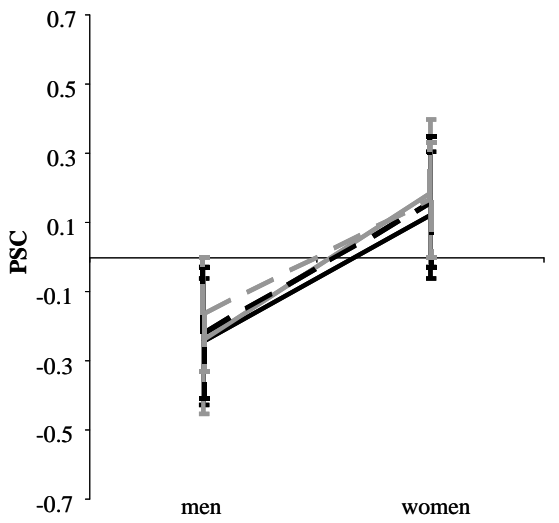

B Gonogo task
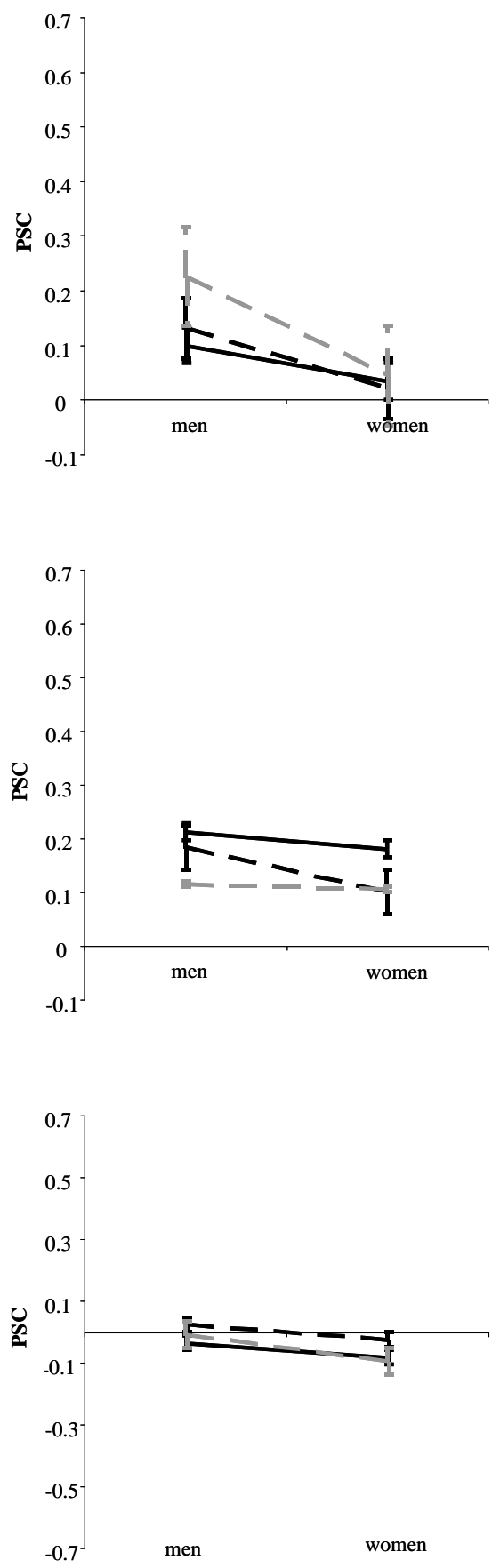
left precuneus (-3 -63 48)
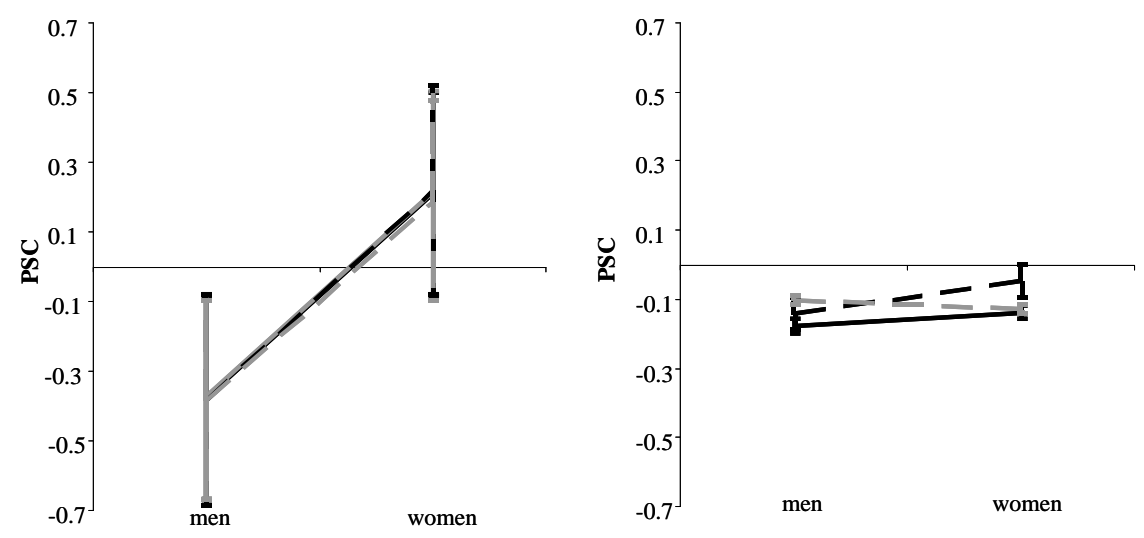

left posterior cingulate sulcus (-9 -30 54)
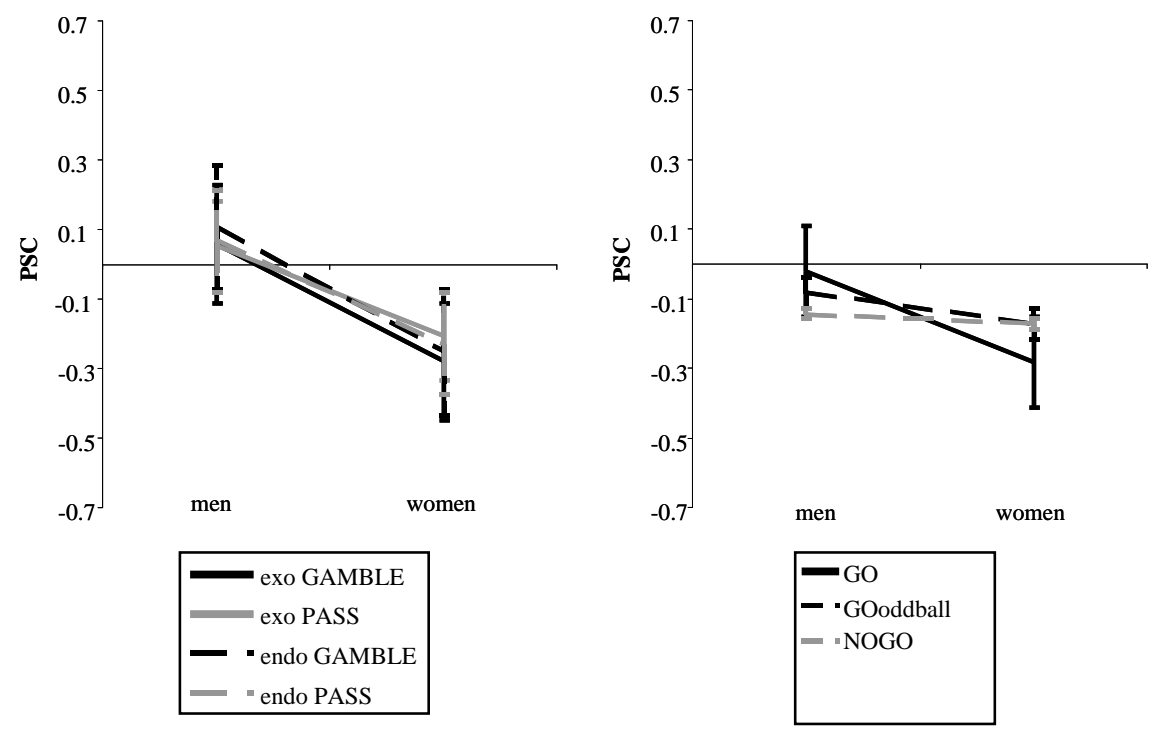

Figure 3: Sex-related activation profiles in ROIs. In each ROI, mean percent signal changes (PSC) were shown for men and women separately, for each task condition: (A) gambling: exogenous GAMBLE and PASS, and endogenous GAMBLE and PASS and (B) gonogo: GO, GOoddball, NOGO. ROIs were selected based on the whole brain results for the pooled gambling task contrast, that differed between men and women. Error bars indicate the standard error of the mean.

Sensation seeking and sex-related brain activation in ROIs

The propensity for sensation seeking behavior might have confounded the presently observed sex differences in brain activation during the gambling task, as men reported a higher sensation seeking tendency compared with women. A 
correlation analysis was performed in each ROI to examine the association between sensation seeking and PSC during the gambling task. Significant correlations were revealed in the anterior and posterior cingulate cortex, and precuneus. In the anterior cingulate cortex, a positive correlation was shown between PSC and sensation seeking for all four gambling conditions (exo GAMBLE $r=.50$, $\mathrm{p}=.005$; exo PASS $\mathrm{r}=.50, \mathrm{p}=.005$; endo GAMBLE $\mathrm{r}=.44, \mathrm{p}=.016$; endo PASS $\mathrm{r}=.46, \mathrm{p}=.010$ ). In the posterior cingulate cluster, sensation seeking was positively correlated with PSC only during the exogenous PASS trials ( $\mathrm{r}=.39$, $\mathrm{p}=.034$ ). In addition, increased sensation seeking was associated with stronger inhibition of the precuneus for all four gambling conditions (exo GAMBLE $r=-$ .47, $\mathrm{p}=.009$; exo PASS $\mathrm{r}=-.48, \mathrm{p}=.007$; endo GAMBLE $\mathrm{r}=-.49, \mathrm{p}=.006$; endo PASS $\mathrm{r}=-.49, \mathrm{p}=.007)$.

We examined the association between sensation seeking and PSC during the gonogo task in each ROI as well. Only in the anterior cingulate cortex, sensation seeking was positively correlated with PSC during NOGO trials ( $\mathrm{r}=.40, \mathrm{p}=.034)$.

In addition, because men and women significantly differed in their sensation seeking score, we examined whether the sex effects in brain activation during gambling performance reported earlier can totally or partially be explained by this difference in sensation seeking. Therefore, we repeated the ANOVA in each ROI for the gambling task with sensation seeking as a covariate. The main effect of sex remained significant in all ROIs except for the anterior cingulate cortex $\left(\mathrm{F}_{(1,28)}=2.31 ; \mathrm{p}=.14\right)$. In line with this finding, the trend for a sex difference in anterior cingulate activation during the gonogo task became non-significant as well $\left(F_{(1,28)}=0.43 ; p=.66\right)$. These findings suggest that, with the exception of the anterior cingulate cortex, sex-related differences in activation during the gambling task are related to sex and not merely mediated by a higher sensation seeking tendency in men.

\section{Discussion}

In the present study we examined sex differences in behavioral performance and brain activation during a gonogo as well as a gambling task. Men and women showed comparable behavioral performance on both decision making tasks. Nevertheless, the present results did show sex effects in brain activation during decision making. We revealed a number of regions in which brain activation differed between men and women during the gambling task in a high motivational context. Such differences, if any, did not reach statistical significance in data recorded in the same participants during an exogenous controlled gonogo task in a low motivational context. This confirms our general starting hypothesis that sex differences in brain function are task-dependent. The sex differences in brain activation were comparable in both the exogenous and endogenous condi- 
tions of the gambling task. This suggests that sex effects are related to the general motivational context of the gambling task, rather than whether decision making responses are exogenous or endogenous controlled.

The BOLD response in two task-related areas was modulated by sex during performance of the gambling task. Men showed a higher BOLD amplitude in both the anterior cingulate cortex and lingual gyrus compared with women. Thus, sex effects occurred in two task specific areas involved in, respectively, cognitive control (e.g. Dosenbach et al., 2007; Sridharan et al., 2008; Stiers et al., 2010) and visual processing of stimuli characteristics (De Luca et al. 2006; Haxby et al., 1994). This finding might suggest that making a decision within a high motivational context takes more effort in male than female young adults. This is in line with the more prolonged structural developmental trajectory of men compared with women (Giedd et al., 1999, 2006). In addition, sex-related activation differences were shown in three other areas that were not task-related. Clusters in inferior frontal sulcus and precuneus were deactivated only by men, whereas a posterior cingulate cluster was only deactivated by women. These three areas have indeed been associated with the so-called default mode network, which is involved in internally directed processes but deactivated during task performance (Greicius et al., 2003; Gusnard \& Raichle, 2001; Sridharan et al., 2008). Regarding the precuneus, however, other studies failed to show its involvement in the default mode network (Buckner et al., 2008; Margulies et al., 2009). Nevertheless, these findings suggest that task-induced deactivation in some areas might follow different patterns in men and women, possibly indicative of different connectivity. In line with our finding, there are some recent suggestions from resting state imaging that sex is a significant determinant of functional connectivity within task positive as well as default mode networks, although the exact contribution of sex requires future research (Biswal et al., 2010; Bluhm et al., 2008).

With respect to sensation seeking, men reported a higher sensation seeking tendency on a self-report questionnaire compared with women. This difference did, however, not influence the behavioral performance on the decision making tasks. In some of the ROIs the sensation seeking score was associated with brain activation during the decision making tasks. In the anterior cingulate cortex, a higher propensity for sensation seeking was associated with enhanced activation during all gambling conditions as well as during nogo trials. Moreover, the higher anterior cingulate activation in male compared with female young adults could be explained by this sex-related difference in sensation seeking. Conversely, in all other ROIs sex-related differences in brain activation during the gambling task were related to sex and not merely mediated by a higher sensation seeking tendency in men. This finding suggests that behavioral tendencies, such as sensation seeking, are important to consider as a third variable in examining 
group differences (i.e. between age groups, sex, or patient versus control groups) in brain activation during decision making.

In sum, the present findings of sex-related activation differences in both task positive and default mode areas during a challenging gambling task indicate that sex is a significant modulator in decision making in a high motivational context. This implicates that including men and women in unequal ratios into groups in fMRI research concerning decision making probably confounds the outcome. Therefore, sex is a subject-related factor that should be considered as a confounder in fMRI data, especially in studies comparing groups. 


\section{References}

Achenbach, T.M., Rescorla, L.A., 2003. Manual for ASEBA adult forms and profiles. Burlington, VT: University of Vermont, Research Centre for Children, Youth and Families.

Amin, Z., Epperson, C.N., Constable, R.T., Canlia, T., 2006. Effects of estrogen variation on neural correlates of emotional response inhibition. Neuroimage, 32, 457-464.

Bell, E.C., Willson, M.C., Wilman, A.H., Dave, S., Silverstonea, P.H., 2006. Males and females differ in brain activation during cognitive tasks. Neuroimage, 30, 529-538.

Bennett, C.M., Miller, M.B., Wolford, G.L., 2009. Neural correlates of interspecies perspective taking in the post-mortem Atlantic Salmon: an argument for multiple comparisons correction. In: Organization for Human Brain Mapping Abstracts.

Biswal, B.B., Mennes, M., Zuob, X., Gohela, S., Kelly, C., Smith, S.M., et al., 2010. Toward discovery science of human brain function, Proceedings of the National Academy of Sciences U.S.A., 107(10), 4734-4739.

Blanton, R.E., Levitt, J.G., Peterson, J.R., Fadale, D., Sporty, M.L., Lee, M., et al., 2004. Gender differences in the left inferior frontal gyrus in normal children. Neuroimage, 22(2), 626-636.

Bluhm, R.L., Osuch, E.A., Lanius, R.A., Boksman, K., Neufeld, R.W.J., Theberge, J., et al., 2008. Default mode network connectivity: effects of age, sex, and analytic approach. Neuroreport, 19(8), 887-891.

Bolla, K.I., Eldreth, D.A., Matochik, J.A., Cadet, J.L., 2004. Sex-related differences in a gambling task and its neurological correlates. Cerebral Cortex, 14, 1226-1232.

Brass, M., von Cramon, D.Y., 2004. Decomposing components of task preparation with functional Magnetic Resonance Imaging. Journal of Cognitive Neuroscience, 16(4), 609-620.

Buckner, R.L., Andrews-Hanna, J.R., Schacter, D.L., 2008. The brain’s default network: Anatomy, function, and relevance to disease. Annals of the New York Academy of Sciences, 1124, 1-38.

Byrnes, J.P., Miller, D.C., Schafer, W.M., 1999. Gender differences in risk taking: A meta analysis. Psychological Bulletin, 125(3), 367-383.

Chikazoe, J., Jimura, K., Asari, T., Yamashita, K., Morimoto, H., Hirose, S., et al., 2009. Functional dissociation in right inferior frontal cortex during performance of go/no-go task. Cerebral Cortex, 19, 146-152.

Dahl, R.E., 2004. Adolescent brain development: a period of vulnerabilities and opportunities. Keynote address. Annals of the New York Academy of Sciences, 1021, 1-22.

De Luca, M., Beckmann, C.F., De Stefano, N., Matthews, P.M., Smith, S.M., 2006. fMRI resting state networks define distinct modes of long-distance interactions in the human brain. Neuroimage, 29, 1359-1367.

Dosenbach, N.U.F, Fair, D.A., Miezin, F.M., Cohen, A.L., Wenger, K.K., Dosenbach, R.A.T., et al., 2007. Distinct brain networks for adaptive and stable task control in humans. Proceedings of the National Academy of Sciences U.S.A., 104(26), 11073-11078.

Duncan, J., Owen, A.M., 2000. Common regions of the human frontal lobe recruited by diverse cognitive demands. Trends in Neurosciences, 23(10), 475-483.

Dunn, L.M., Dunn, L.M., 2005. Peabody Picture Vocabulary Test-III-NL. Handleiding [Manual]. Amsterdam: Harcourt Assessment B.V.

Fernandez, G., Weis, S., Stoffel-Wagner, B., Tendolkar, I., Reuber, M., Beyenburg, S., et al., 2003. Menstrual cycle-dependent neural plasticity in the adult human brain is hormone, task, and region specific. The Journal of Neuroscience, 23(9), 3790-3795. 
Friston, K.J., Williams, S., Howard, R., Frackowiak, R.S.J., Turner, R., 1996. Movementrelated effects in fMRI time-series. Magnetic Resonance in Medicine, 35(3), 346-355.

Gardner, M., Steinberg, L., 2005. Peer influence on risk taking, risk preference, and risky decision making in adolescence and adulthood: an experimental study. Developmental Psychology, 41(4), 625-635.

Genovese, C.R., Lazar, N.A., Nicols, T., 2002. Thresholding of statistical maps in functional neuroimaging using the false discovery rate. NeuroImage, 15, 870-878.

Giedd, J.N., Blumenthal, J., Jeffries, N.O., Castellanos, F.X., Liu, H., Zijdenbos, A., et al., 1999. Brain development during childhood and adolescence: a longitudinal MRI study. Nature Neuroscience, 2, 861-863.

Giedd, J.N., Clasen, L.S., Lenroot, R., Greenstein, D., Wallace, G.L., Ordaz, S., et al., 2006. Puberty-related influences on brain development. Molecular and Cellular Endocrinology, 254-255, 154-162.

Good, C.D., Johnsrude, I., Ashburner, J., Henson, R.N.A., Friston, K.J., Frackowiak, R.S.J., 2001. Cerebral asymmetry and the effects of sex and handedness on brain structure: A voxel-based morphometric analysis of 465 normal adult human brains. Neuroimage, 14, 685-700.

Greicius, M.D., Krasnow, B., Reiss, A.L., Menon, V., 2003. Functional connectivity in the resting brain: A network analysis of the default mode hypothesis. Proceedings of the National Academy of Sciences U.S.A., 100(1), 253-258.

Gusnard, D.A., Raichle, M.E., 2001. Searching for a baseline: Functional imaging and the resting human brain. Nature Reviews Neuroscience, 2, 685-694.

Halari, R., Kumari, V., 2005. Comparable cortical activation with inferior performance in women during a novel cognitive inhibition task. Behavioural Brain Research, 158, 167173.

Hampshire, A., Chamberlain, S.R., Monti, M.M., Duncan, J., Owen, A.M., 2010. The role of the right inferior frontal gyrus: inhibition and attentional control. NeuroImage, 50(3), 1313-1319.

Haut, K.M., Barch, D.M., 2006. Sex influences on material-sensitive functional lateralization in working and episodic memory: Men and women are not all that different. Neuroimage, 32, 411-422.

Haxby, J.V., Horwitz, B., Ungerleider, L.G., Maisog, J.M., Pietrini, P., Grady, C.L., 1994. The functional organization of human extrastriate cortex: A PET-rCBF study of selective attention to faces and locations. The Journal of Neuroscience, 74(11), 6336-6353.

Hoyle, R.H., Stephenson, M.T., Palmgreen, P., Lorch, E.P., Donohew, R.L., 2002. Reliability and validity of a brief measure of sensation seeking. Personality and Individual Differences, 32, 401-414.

Johnstone, T., Ores Walsh, K.S., Greischar, L.L., Alexander, A.L., Fox, A.S., Davidson, R., et al., 2006. Motion correction and the use of motion covariates in multiple-subject fMRI analysis. Human Brain Mapping, 27, 779-788.

Kimura, D., 1996. Sex, sexual orientation and sex hormones influence human cognitive function. Current Opinion Neurobiology, 6, 259-263.

Kucian, K., Loenneker, T., Dietrich, T., Martin, E., von Aster, M., 2005. Gender differences in brain activation patterns during mental rotation and number related cognitive tasks. Psychology Science, 47(1), 112-131.

Lee, T.M.C., Chan, C.C.H., Leung, A.W.S., Fox, P.T., Gao, J., 2009. Sex-related differences in neural activity during risk taking: An fMRI study. Cerebral Cortex, 19, 1303-1312.

Leonard, C.M., Towler, S., Welcome, S., Halderman, L.K., Otto, R., Eckert, M.A., et al., 2008. Size matters: cerebral volume influences sex differences in neuroanatomy. Cerebral Cortex, 18, 2920-2931. 
Li, C.S., Huang, C., Constable, R.T., Sinha, R., 2006. Gender differences in the neural correlates of response inhibition during a stop signal task. Neuroimage, 32, 1918-1929.

Linn, M.C., Petersen, A.C., 1985. Emergence and characterization of gender differences in spatial abilities: A meta-analysis. Child Development, 56, 1479-1498.

Luders, E., Gaser, C., Narr, K.L., Toga, A.W., 2009. Why sex matters: Brain size independent differences in gray matter distributions between men and women. The Journal of Neuroscience, 29(45), 14265-14270.

Luders, E., Narr, K.L., Thompson, P.M., Woods, R.P., Rex, D.E., Jancke, L. et al., 2005. Mapping cortical gray matter in the young adult brain: Effects of gender. Neuroimage, 26, 493-501.

Manes, F., Sahakian, B., Clark, L., Rogers, R., Antoun, N., Aitken, M., et al., 2002. Decision making processes following damage to the prefrontal cortex. Brain, 125, 624-639.

Margulies, D.A., Vincent, J.L., Kelly, C., Lohmann, G., Uddin, L.Q., Biswal, B.B., et al., 2009. Precuneus shares intrinsic functional architecture in humans and monkeys. Proceedings of the National Academy of Sciences U.S.A., 106(47), 20069-20074.

Mayer, A.R., Franco, A.R., Ling, J., Canive, J.M., 2007. Assessment and quantification of head motion in neuropsychiatric functional imaging research as applied to schizophrenia. Journal of the International Neuropsychological Society, 13, 839-845.

Mennes, M., Van den Bergh, B., Lagae, L., Stiers, P., 2009. Developmental brain alterations in 17 year old boys are related to antenatal maternal anxiety. Clinical Neurophysiology, 120, 1116-1122.

Mennes, M., Wouters, H., van den Bergh, B., Lagae, L., Stiers, P., 2008. ERP correlates of complex human decision making in a gambling paradigm: Detection and resolution of conflict. Psychophysiology, 45, 714-720.

Miller, E.K., 2000. The prefrontal cortex and cognitive control. Nature Reviews Neuroscience, 1, 59-65.

Nelson, E.E., Leibenluft, E., McClure, E.B., Pine, D.S., 2005. The social re-orientation of adolescence: A neuroscience perspective on the process and relation to psychopathology. Psychological Medicine, 35, 163-174.

O’Doherty, J., Kringelbach, M.L., Rolls, E.T., Hornak, J., Andrews, C., 2001. Abstract reward and punishment representations in the human orbitofrontal cortex. Nature Neuroscience, 4, 95-102.

Paulus, M.P., 2005. Neurobiology of decision-making: Quo vadis? Cognitive Brain Research, 23, 2-10.

Paus, T., Keshavan, M., Giedd, J.N., 2008. Why do many psychiatric disorders emerge during adolescence? Nature Reviews Neuroscience, 9, 947-957.

Raven, J., Raven, J.C., Court, J.H., 1998. Manual for Raven’s Progressive Matrices and Vocabulary Scales. Section 1: General Overview. Harcourt Assessment, San Antonio TX.

Reavis, R., Overman, W.H., 2001. Adult sex differences on a decision making task previously shown to depend on the orbital prefrontal cortex. Behavioral Neuroscience, 115, 196206.

Rosenblitt, J.C., Soler, H., Johnson, S.E., Quadagno, D.M., 2001. Sensation seeking and hormones in men and women: exploring the link. Hormones and Behavior, 40, 396-402.

Schlosser, R., Hutchinson, M., Joseffer, S., Rusinek, H., Saarimaki, A., Stevenson, J., et al., 1998. Functional magnetic resonance imaging of human brain activity in a verbal fluency task. Journal of Neurology, Neurosurgery \& Psychiatry, 64, 492-498.

Shaywitz, B.A., Shaywitz, S.E., Pugh, K.R., Constable, R.T., Skudlarski, P., Fulbright, R.K., et al., 1995. Sex differences in the functional organization of the brain for language. Nature, 373, 607-609. 
Sisk, C.L., Zehr, J.L., 2005. Pubertal hormones organize the adolescent brain and behavior. Frontiers in Neuroendocrinology, 26, 163-174.

Sowell, E.R., Peterson, B.S., Kan, E., Woods, R.P., Yoshii, J., Bansal, R., et al., 2007. Sex differences in cortical thickness mapped in 176 healthy individuals between 7 and 87 years of age. Cerebral Cortex, 17, 1550-1560.

Sowell, E.R., Thompson, P.M., Holmes, C.J., Jernigan, T.L., Toga, A.W., 1999. In vivo evidence for post-adolescent brain maturation in frontal and striatal regions. Nature neuroscience, 2(10), 859-861.

Speck, O., Ernst, T., Braun, J., Koch, C., Miller, E., Chang, L., 2000. Gender differences in the functional organization of the brain for working memory. Neuroreport, 11, 25812585.

Sridharan, D., Levitin, D.J., Menon, V., 2008. A critical role for the right fronto-insular cortex in switching between central-executive and default-mode networks. Proceedings of the National Academy of Sciences U.S.A., 105(34), 12569-12574.

Steinberg, L., 2008a. A social neuroscience perspective on adolescent risk-taking. Developmental Review, 28, 78-106.

Steinberg, L., Albert, D., Cauffman, E., Banich, M., Graham, S., Woolard, J., 2008b. Age differences in sensation seeking and impulsivity as indexed by behavior and self report: Evidence for a dual systems model. Developmental Psychology, 44(6), 1764-1778.

Stiers, P., Mennes, M., Sunaert, S., 2010. Distributed task coding throughout the multiple demand network of the human frontal-insular cortex, NeuroImage, 52(1), 252-262.

Talairach, J., Tournoux, P., 1988. Co-planar stereotaxic atlas of the human brain. Thieme Medical Publishers Inc, New York.

Unterrainer, J.M., Wranek, U., Staffen, W., Gruber, T., Ladurner, G., 2000. Lateralized cognitive visuospatial processing: is it primarily gender related or due to quality of performance? A HMPAO-SPECT study. Neuropsychobiology, 41, 95-101.

Van der Elst, W., Van Boxtel, M.P., Van Breukelen, G.J., Jolles, J., 2006. The Stroop colorword test: influence of age, sex, and education; and normative data for a large sample across the adult age range. Assessment, 13, 62-79.

Voyer, D., Voyer, S., Bryden, M.P., 1995. Magnitude of sex differences in spatial abilities: A meta-analysis and consideration of critical variables. Psychological Bulletin, 117(2), 250-270.

Weiss, E., Siedentopf, C.M., Hofer, A., Deisenhammer, E.A., Hoptman, M.J., Kremser, C., et al., 2003. Sex differences in brain activation pattern during a visuospatial cognitive task: a functional magnetic resonance imaging study in healthy volunteers. Neuroscience Letters, 344, 169-172.

Yoo, S., Choi, B., Juh, R., Pae, C., Lee, C., 2005. Head motion analysis during cognitive fMRI examination: Application in patients with schizophrenia. Neuroscience Research, 53, 84-90.

Zuckerman, M., Eysenck, S., Eysenck, H.J., 1978. Sensation seeking in England and America: cross-cultural age and sex comparisons. Journal of Consulting and Clinical Psychology, 46, 139-149. 

Chapter

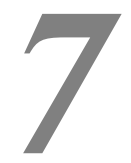

Concluding remarks 
Adolescence is characterized by developmental gains in the physical, cognitive and social domain. By contrast, adolescents are also prone to risk taking behavior and an increased incidence of psychiatric disorders and mortality. These features have increasingly been explained by prolonged maturational changes in the structure and organization of the adolescent brain. However, the implications of structural brain maturation at the level of brain activation underlying these behavioral and cognitive changes are still largely unknown. Furthermore, previous developmental findings might be confounded by subject-related and methodological factors. The studies described in this thesis aspired to contribute to the current scientific knowledge by examining neurocognitive development and its underlying age-related functional changes in the age window of adolescence, and by investigating the effect of various subject-related factors on this neurocognitive development. In this final chapter, general findings of these empirical studies are discussed in the light of the thesis aims. Furthermore, theoretical implications as well as recommendations for future research will be discussed.

\section{Handling methodological confounders in developmental fMRI studies}

Previous developmental fMRI findings might be contaminated by uncontrolled subject characteristics and methodological confounders. Regarding subject characteristics, for example, some fMRI studies included adolescents within a broad age range of 9 to 17 years, and compared them with adults (Eshel et al., 2007; Rubia et al., 2006). This yields a highly heterogeneous adolescent group, since the contribution of structural brain development, socio-emotional and pubertal changes are highly variable within this age range (Giedd et al., 2006, 2009; Nelson et al., 2005). This higher heterogeneity biases the analyses towards revealing weaker activations in the adolescent group. Head motion is an example of an age-dependent methodological confounder in fMRI studies. Unmodeled signal variation due to head motion decreases the sensitivity of fMRI analysis (Friston et al., 1996; Johnstone et al., 2006). Given that a higher amount of head motion occurs in younger age groups compared with adults (chapter 3 and 4), this leads to reduced activation in the younger groups. Another methodological confounder in developmental fMRI studies is age-related variability in anatomical and functional organization (e.g. Uylings et al., 2005; Wilke \& Holland, 2003). Group averaging in fMRI might lead in developing subjects to a decreased colocalization of cortical areas, resulting in reduced or more diffuse activation (Berl et al., 2006; Manoach, 2003). Therefore, controlling for such ageentangled confounding variables as described here is a fundamental requirement in establishing developmental changes in brain activation. In the fMRI studies described in this thesis (chapter 3 to 6), we controlled for these confounding variables more rigorously than any previous study. First, we included only male 
participants within narrow age ranges of respectively 12-13, 16-17 and 20-21 years, who were in addition comparable regarding intelligence and parental level of education. Second, age groups were equated on the amount of head motion and sudden head movements were explicitly modelled such that we only examined task trials not contaminated with head motion. In addition, we included realignment parameters in the statistical analyses to model signal variation due to motion. Lastly, in chapter 3 we performed region of interest (ROI) analyses by identifying ROIs within each individual's data analysis instead of within the averaged group data. In this way, the developmental fMRI findings in the present thesis cannot be accounted for by subject heterogeneity or age-related differences in either head motion or anatomical variability.

Psychological stress during fMRI scanning might confound age effects in brain activation as well, as fMRI might be more stressful for children and adolescents compared with adults (Eatough et al., 2009). In chapter 5 we investigated whether cortisol reactivity during fMRI scanning was age-dependent and influenced fMRI results. Although both young and old adolescents showed an anticipatory stress response during fMRI scanning, cortisol was associated with the BOLD response only in young adolescents. This finding implies that the effect of cortisol on brain activation might be age-dependent. Therefore, cortisol reactivity to fMRI scanning should be considered as confounder and incorporated in analysing developmental fMRI data as well.

\section{Functional brain maturation}

In chapter 3 and 4 we investigated age-related functional changes underlying neurocognitive development, while rigorously controlling for confounding factors as discussed above. We compared functional activation patterns between different adolescent age groups during the performance of, respectively, a gambling and a gonogo task. In general, 13, 17 and 21 year-olds activated similar brain regions during task execution. In line with this finding, age effects were rather subtle in contrast to the robust activations revealed during task performance. Consequently, age-related functional differences were often not picked up by the traditional, univariate general linear model, especially not when the recommended multiple comparisons correction was applied. However, by using more sensitive methods we were able to elicit developmental effects in brain activation. In chapter 3, for instance, the individually determined ROI-based age group comparison was able to reveal age-related changes in signal strength in brain areas activated during performance of the gambling task. The BOLD magnitude in areas of task positive networks increased between ages 13 and 21. In addition, task-induced deactivation of some default mode areas was also en- 
hanced with increasing age. These developmental changes in both task positive and default mode areas were more pronounced during difficult task conditions.

In chapter 4, we applied multivariate pattern classification analysis to reveal developmental changes in brain activation during a simple gonogo task. This method might be more sensitive than the univariate approach (De Martino et al., 2008). Given age-related changes in the configuration of functional brain networks (Fair et al., 2007, 2008), it makes sense that studying development in terms of changing activation patterns is more sensitive than studying changes in individual voxels (Bray et al., 2009). Based on widespread maturational changes in anatomy (e.g. Giedd et al., 2009) and functional connectivity (e.g. Fair et al., 2007, 2008) during adolescence, we asked whether functional maturation involved widespread changes in brain responsiveness as well, and whether these functional changes were specific to a particular cognitive function or not. The multivariate pattern classification was indeed highly accurate in discriminating between 13 year-olds and respectively 17 and 21 year-olds based on their activation patterns during a simple gonogo task. This suggests that developmental changes are spatially distributed throughout the brain, affecting the responsiveness of a wide range of cortical structures. The age-affected voxels were roughly confined to task-related areas. By using ROI-based age group comparisons in these areas, we could confirm the main developmental pattern in signal strength observed in our gambling study (chapter 3), namely an enhanced BOLD amplitude in task positive areas complemented with enhanced task-induced deactivation of default mode areas. Moreover, we showed that this distributed agedistinctive pattern learned by the classifier is not specific to a particular cognitive process, but generalizes over the different conditions of the gonogo task. Moreover, the classifier trained on the gonogo data was even able to accurately predict age group based on activation patterns during a different cognitive task, i.e. the gambling task, performed by the same participants. This shows that the functional effect of brain maturation seems to be a general developmental pattern, not specific to the particular neurocognitive functions triggered by different tasks.

Based on the results of chapter 3 and 4, functional brain maturation can be conceptualized as subtle but widespread changes in functional responsiveness of both task positive and default mode areas in the brain, which are independent of specific neurocognitive functions. First, these age-related functional changes might reflect anatomical maturation of these areas. Indeed, region specific changes in cortical density have been observed, with prolonged maturation of dorsal parietal, dorsal and medial prefrontal areas during and post adolescence (Gogtay et al., 2004; Sowell et al., 1999, 2001). Second, the present findings fit with maturation related changes in connectivity within several functional networks distributed throughout the brain, as revealed by recent resting state imaging findings. Some of these functional networks are involved in neurocognitive 
processes (task positive networks), whereas the default mode network is involved in internally directed processes but is deactivated during task performance (e.g. Dosenbach et al., 2007; Gusnard \& Raichle, 2001; Sridharan et al., 2008). These networks continue to specialize and reorganize throughout adolescence, becoming more distinct and segregated from one another and further integrating long range connections (Fair et al., 2007, 2008; Kelly et al., 2009). Although different age groups activate the same areas within task positive networks as well as deactivate the same default mode areas during task performance, the way these areas are functionally connected becomes more mature and efficient with increasing age. The activation of a more mature and optimal network with increasing age might enable computational abilities of the system to support higher levels of activity that are not possible in an immature network (Luna et al., 2010). For instance, lower or even the absence of connectivity between functional network areas might impede access to a region or another network that is needed for executing a neurocognitive function. Moreover, the complementary age-related functional changes in task positive as well as default mode networks suggest that the anti-correlation between both networks (Fox et al., 2005) might be subject to maturation as well. In adults, higher anticorrelations between both networks have been associated with higher taskinduced BOLD activation (Mennes et al., 2010) as well as with less variable behavioral performance (Kelly et al., 2008). Future research that examines the associations between maturational changes in functional connectivity and agerelated changes in brain activation during neurocognitive tasks is needed to reveal whether more mature functional networks are indeed underlying the agerelated functional changes described here.

\section{The influence of male/female sex on neurocognitive development}

In chapter 2 and 6 we examined the influence of sex on behavioral performance as well as brain activation during socio-emotional and cognitive functions. We hypothesized that sex-related differences would exist, based on differential trajectories of structural brain development in male and female adolescents (e.g. Lenroot \& Giedd, 2010), as well as sex differences in performance on various neurocognitive tasks (e.g. Bolla et al., 2004; Garavan et al., 2006). The behavioral results of chapter 2 supported a sex-related modulation of mentalizing speed, independent of the specific adolescent age group. Male adolescents needed more time than female adolescents to evaluate and judge hypothetical real-life situations. The advantage of female adolescents on the mentalizing tasks is in line with previous findings of girls outperforming boys on social tasks as well as tasks including a large verbal component (e.g., Baron-Cohen, 2002; Wassenberg et al., 2008). The fMRI study described in chapter 6 examined sex- 
related differences in functional activation patterns during decision making. Sex effects in brain activation were revealed during a gambling task with a high motivational context, but not during a gonogo task (low motivational context). In two task positive areas men showed an increased BOLD amplitude compared with women, which might suggest that motivational decision making takes more effort in male than female young adults. In addition, some task-induced deactivations might follow different patterns in men and women. Thus, both studies provided evidence for a sex-related modulation on respectively behavioral performance and functional activation during neurocognitive tasks, more specifically regarding mentalizing and motivational decision making. These findings imply that sex is an important subject-related factor that should be considered in studying neurocognitive development and its underlying neural correlates.

\section{Theoretical implications and recommendations}

The results described in this thesis have several implications for theoretical frameworks concerning functional brain maturation as well as future studies into developmental differences in brain activation. Previous developmental fMRI findings should be interpreted in the light of the possible confounders revealed in the present thesis. For instance, while rigorously controlling for confounding factors we found in chapter 3 only partial evidence for the focalization hypothesis, which has often been presented as a possible model for functional development (Durston \& Casey, 2006; Durston et al., 2006). We did find evidence for the hypothesized enhanced BOLD response in task-related areas with increasing age, but this was neither accompanied by age-related changes in the number of active voxels nor by a qualitative shift in brain areas involved in task execution as hypothesized. In addition, this age-related increased BOLD response in taskrelated areas was inconsistent with previous findings of a decreased BOLD response with age (e.g. Booth et al., 2003; Galvan et al., 2006). Therefore, the developmental findings described in this thesis do not support explanations for this decreased signal strength as reflecting either more efficient neural processing with age, or greater effort in younger subjects to perform the same task. Earlier evidence for an age-related decrease in activation extent (e.g. Durston et al., 2006; Luna et al., 2001), as well as for an age-related decrease in BOLD magnitude (e.g. Booth et al., 2003; Galvan et al., 2006) might have been mediated by confounding variables. In line with this, future studies into age-related functional changes are recommended to control for several confounding factors. First, subject heterogeneity should be decreased by using strict selection criteria concerning age ranges, sex and pubertal phase. Second, methodological factors, such as stress reactivity as well as head motion during scanning, can contaminate developmental fMRI findings and should therefore be controlled for. 
The developmental fMRI findings described in this thesis showed that age effects in functional activation are rather subtle. Given the subtlety of age effects relative to the variability in measured BOLD responses, traditional univariate analyses proved to lack the sensitivity to reveal these maturational changes in functional activation. Therefore, developmental neuroimaging studies should include larger sample sizes than are presently used in order to achieve appropriate power to detect subtle developmental differences. In addition, more sensitive methods should be considered such as individually determined region of interest analysis (chapter 3) or multivariate pattern classification analysis (chapter 4). In this thesis, multivariate pattern classification was applied for the first time in a developmental context with the intention to classify subjects based on functional activation maps. We showed that this multivariate approach could highly accurate classify different adolescent age groups with high accuracy, suggesting that this might be a very promising tool for future research comparing groups.

The implications and recommendations concerning developmental fMRI studies discussed here, may also have great value for fMRI studies in the context of aging, group comparisons (i.e. patient versus control), and inter-individual differences.

\section{Conclusion}

Based on the results from our experimental studies we conclude that this thesis has contributed to our understanding of age-related functional changes that underlie neurocognitive development in the age window of adolescence. In our fMRI studies we controlled for subject-related and methodological confounders, in order to examine true age-related effects in brain activation. This methodological rigorous approach provided new insight into the relevance of specific confounders for developmental fMRI results that were ignored or uncontrolled in previous studies. Based on the findings described in this thesis, functional brain maturation can be conceptualized as subtle but widespread changes in functional responsiveness of task-related voxels. The BOLD magnitude in task positive areas in enhanced between ages 13 and 21, complemented by an increased task-induced deactivation of default mode areas. Moreover, this distributed age-distinctive pattern of brain activation seemed applicable to a range of neurocognitive processes, indicating a general developmental principle. In addition, this thesis provided evidence for the importance of the factors male/female sex as well as stress reactivity to scanning on this neurocognitive development. In all, the findings described here underscore the notion that research into agerelated changes in brain activation underlying neurocognitive development in adolescence should take subject-related and methodological factors into consideration. 


\section{References}

Baron-Cohen, S., 2002. The extreme male brain theory of autism. Trends in Cognitive Sciences, 6(6), 248-254.

Berl, M.M., Vaidya, C.J., Gaillard, W.D., 2006. Functional imaging of developmental and adaptive changes in neurocognition. NeuroImage, 30, 679-691.

Bolla, K.I., Eldreth, D.A., Matochik, J.A., Cadet, J.L., 2004. Sex-related differences in a gambling task and its neurological correlates. Cerebral Cortex, 14, 1226-1232.

Booth, J.R., Burman, D.D., Meyer, J.R., Lei, Z., Trommer, B.L., Davenport, N.D., et al., 2003. Neural development of selective attention and response inhibition. NeuroImage, 20, 737-751.

Bray, S., Chang, C., Hoeft, F., 2009. Applications of multivariate pattern classification analyses in developmental neuroimaging of healthy and clinical populations. Frontiers in Human Neuroscience, 3, 1-12.

De Martino, F., Valente, G., Staeren, N., Ashburner, J., Goebel, R., Formisano, E., 2008. Combining multivariate voxel selection and support vector machines for mapping and classification of fMRI spatial patterns. NeuroImage, 43, 44-58.

Dosenbach, N.U.F, Fair, D.A., Miezin, F.M., Cohen, A.L., Wenger, K.K., Dosenbach, R.A.T., et al., 2007. Distinct brain networks for adaptive and stable task control in humans. Proceedings of the National Academy of Sciences U.S.A., 104(26), 11073-11078.

Durston, S., Casey. B.J., 2006. What have we learned about cognitive development from neuroimaging? Neuropsychologia, 44, 2149-2157.

Durston, S., Davidson, M.C., Tottenham, N., Galvan, A., Spicer, J., Fossella, J.A., et al., 2006. A shift from diffuse to focal cortical activity with development. Developmental Science, 9(1), 1-20.

Eatough, E.M., Shirtcliff, E.A., Hanson, J.L., Pollak, E.A., 2009. Hormonal reactivity to MRI scanning in adolescents. Psychoneuroendocrinology 34, 1242-1246.

Eshel, N., Nelson, E.E., Blair, J., Pine, D.S., Ernst, M., 2007. Neural substrates of choice selection in adults and adolescents: development of the ventrolateral prefrontal and anterior cingulate cortices. Neuropsychologia, 45, 1270-1279.

Fair, D.A., Cohen, A.L., Dosenbach, N.U.F., Church, J.A., Miezin, F.M., Barch, D.M., et al., 2008. The maturing architecture of the brain's default network. Proceedings of the National Academy of Sciences U.S.A., 105(10), 4028-4032.

Fair, D.A., Dosenbach, N.U.F., Church, J.A., Cohen, A.L., Brahmbhatt, S., Miezin, F.M., et al., 2007. Development of distinct control networks through segregation and integration. Proceedings of the National Academy of Sciences U.S.A., 104, 13507-13512.

Fox, M.D., Snyder, A.Z., Vincent, J.L., Corbetta, M., Van Essen, D.C., Raichle, M.E., 2005. The human brain is intrinsically organized into dynamic, anticorrelated functional networks. Proceedings of the National Academy of Sciences U.S.A., 102, 9673-9678.

Friston, K.J., Williams, S., Howard, R., Frackowiak, R.S.J., Turner, R., 1996. Movementrelated effects in fMRI time-series. Magnetic Resonance in Medicine, 35(3), 346-355.

Galvan, A., Hare, T.A., Parra, C.E., Penn, J., Voss, H., Glover, G., et al., 2006. Earlier development of the accumbens relative to orbitofrontal cortex might underlie risk-taking behavior in adolescents. The Journal of Neuroscience, 26(25), 6885-6892.

Garavan, H., Hester, R., Murphy, K., Fassbender, C., Kelly, C., 2006. Individual differences in the functional neuroanatomy of inhibitory control. Brain Research, 1105, 130-142. 
Giedd, J.N., Clasen, L.S., Lenroot, R., Greenstein, D., Wallace, G.L., Ordaz, S., et al., 2006. Puberty-related influences on brain development. Molecular and Cellular Endocrinology, 254-255, 154-162.

Giedd, J.N., Lalonde, F.M., Celano, M.J., White, S.L., Wallace, G.L., Lee, N.R. et al., 2009. Anatomical brain magnetic resonance imaging of typically developing children and adolescents. Journal of the American Academy of Child and Adolescent Psychiatry, 48(5), 465-470.

Gogtay, N., Giedd, J.N., Lusk, L., Hayashi, K.M., Greenstein, D., Vaituzis, A.C., et al., 2004. Dynamic mapping of human cortical development during childhood through early adulthood. Proceedings of the National Academy of Sciences U.S.A., 101(21), 81748179.

Gusnard, D.A., Raichle, M.E., 2001. Searching for a baseline: Functional imaging and the resting human brain. Nature Reviews Neuroscience, 2, 685-694.

Johnstone, T., Ores Walsh, K.S., Greischar, L.L., Alexander, A.L., Fox, A.S., Davidson, R.., et al., 2006. Motion correction and the use of motion covariates in multiple-subject fMRI analysis. Human Brain Mapping, 27, 779-788.

Kelly, A.M.C., Di Martino, A., Uddin, L.Q., Shehzad, Z., Gee1, D.G., Reiss, P.T., et al., 2009. Development of anterior cingulate functional connectivity from late childhood to early adulthood. Cerebral Cortex, 19, 640-657.

Kelly, A.M.C., Uddin, L.Q., Biswal, B.B., Castellanos, F.X., Milham, M.P., 2008. Competition between functional brain networks mediates behavioral variability. Neuroimage, 39, 527-537.

Lenroot, R.K., Giedd, J.N., 2010. Sex differences in the adolescent brain. Brain and Cognition, 72(1), 46-55.

Luna, B., Padmanabhan, A., O’Hearn, K., 2010. What has fMRI told us about the Development of Cognitive Control through Adolescence? Brain and Cognition, 72(1), 101-113.

Luna, B., Thulborn, K.R., Munoz, D.P., Merriam, E.P., Garver, K.E., Minshew, N.J., et al., 2001. Maturation of widely distributed brain function subserves cognitive development. NeuroImage, 13, 786-793.

Manoach, D.S., 2003. Prefrontal cortex dysfunction during working memory performance in schizophrenia: reconciling discrepant findings. Schizophrenia Research, 60, 285-298.

Mennes, M., Kelly, C., Zuo, X., Di Martino, A., Biswal, B.B., Castellanos, F.X. et al., 2010. Inter-individual differences in resting-state functional connectivity predict task-induced BOLD activity. NeuroImage, 50(4), 1690-1701.

Nelson, E.E., Leibenluft, E., McClure, E.B., Pine, D.S., 2005. The social re-orientation of adolescence: A neuroscience perspective on the process and relation to psychopathology. Psychological Medicine, 35, 163-174.

Rubia, K., Smith, A.B., Woolley, J., Nosarti, C., Heyman, I., Taylor, E., et al., 2006. Progressive increase of frontostriatal brain activation from childhood to adulthood during event-related tasks of cognitive control. Human Brain Mapping, 27, 973-993.

Sowell, E.R., Thompson, P.M., Holmes, C.J., Jernigan, T.L., Toga, A.W., 1999. In vivo evidence for post-adolescent brain maturation in frontal and striatal regions. Nature neuroscience, 2(10), 859-861.

Sowell, E.R., Thompson, P.M., Tessner, K.D., Toga, A.W., 2001. Mapping continued brain growth and gray matter density reduction in dorsal frontal cortex: Inverse relationships during postadolescent brain maturation. The Journal of Neuroscience, 21(22), 88198829.

Sridharan, D., Levitin, D.J., Menon, V., 2008. A critical role for the right fronto-insular cortex in switching between central-executive and default-mode networks. Proceedings of the National Academy of Sciences U.S.A., 105(34), 12569-12574. 
Uylings, H.B.M., Rajkowsk, G., Sanz-Arigita, E., Amunts, K., Zilles, K., 2005. Consequences of large interindividual variability for human brain atlases: converging macroscopical imaging and microscopical neuroanatomy. Anatomy \& Embryology, 210, 423-431.

Wassenberg, R., Hurks, P.P., Hendriksen, J.G., Feron, F.J., Meijs, C.J., Vles, J.S., et al., 2008. Age-related improvement in complex language comprehension: Results of a crosssectional study with 361 children aged 5 to 15. Journal of Clinical and Experimental Neuropsychology, 30(4), 435-448.

Wilke, M., Holland, S.K., 2003. Variability of gray and white matter during normal development: a voxel-based MRI analysis. NeuroReport, 14(15), 1887-1890. 
Adolescence has been described as a period of profound developmental changes in behavior, neurocognitive functions and brain structure. However, the agerelated changes in functional brain activation underlying neurocognitive improvement are still unknown. Moreover, previous developmental results might be contaminated with uncontrolled subject characteristics as well as methodological confounders, such as head motion and stress reactivity during scanning. The first aim of this thesis was to study neurocognitive development in the age window of adolescence, and to examine age-related changes in brain activation underlying this development. Two large-scale cross-sectional studies were conducted to achieve this aim. Various age groups within adolescence were compared on both behavioral performance and functional activation patterns during neurocognitive tasks. The second aim of this thesis was to investigate the effect of subject-related and methodological factors on these developmental trajectories. Differences between male and female adolescents were investigated as the major subject-related factor, and stress-reactivity to fMRI scanning was the major methodological factor investigated. Furthermore, we controlled for the typical confounding variables within developmental fMRI studies. In chapter 1, a general introduction to this thesis was given.

In chapter 2, we examined developmental trajectories of emotional versus cognitive functions in a sample of adolescents aged 13-19. Both mentalizing speed regarding emotions, and mentalizing speed regarding actions as well as the planning time on the Tower task improved linearly with age into late adolescence. No differential trajectories were found for emotional versus cognitive development. In addition, two subject-related factors, i.e. sex and pubertal phase, proved to influence mentalizing speed. Girls outperformed boys on both mentalizing versions, and a later pubertal phase measured in boys was associated with increased mentalizing speed.

Developmental fMRI results have suggested a progression in focalization, characterized by an age-related decrease in activation extent as well as an increased signal strength in task-related areas. We evaluated this hypothesis in chapter 3, while controlling for several confounders that might bias towards focalization. Male adolescents, selected within small age ranges, of respectively 13, 17 and 21 years old performed a challenging gambling task. We incorporated head motion corrections and identified regions of interest for each participant separately to rule out the influence of age-related anatomical variability. Although different adolescent age groups engaged similar brain areas during 
decision making, the response magnitude in these areas was modulated by age. The BOLD amplitude in task positive areas increased with age, complemented by an increased deactivation of default mode areas, both more pronounced during difficult gambling conditions. The age-related increased BOLD response was, however, neither accompanied by an age-related decrease in activation extent, nor by any qualitative shift in activated areas as hypothesized. So, while controlling for confounding factors we only found evidence for the focalization of activation into task-specific brain areas with increasing BOLD response between ages 13 and 21.

In chapter 4, we examined whether age-related changes in brain activation were widespread throughout the brain, and whether this pattern was specific to a particular cognitive function or not. This view on functional maturation is based on widespread maturational changes in anatomy and functional connectivity, and contrasts with previous developmental fMRI studies that focussed on functional changes associated with particular changes in behavior. Next to the traditional, univariate voxel-wise analysis, we applied multivariate pattern classification analysis to study developmental changes in brain activation during a simple gonogo task. The multivariate approach was highly accurate in discriminating between 13 year-olds and respectively 17 and 21 year-olds. This suggests that developmental changes are spatially distributed throughout the brain, affecting the responsiveness of a wide range of task positive and default mode regions. Moreover, we showed that this developmental pattern is independent of the specific cognitive function performed, since it can be accurately generalized across different gonogo conditions and even to a totally different gambling task performed by the same participants. In contrast to the multivariate results, univariate voxel-wise analysis revealed few age-related clusters that only differed between 13 and 21 year-olds. This suggests that a multivariate approach might be more suited for studying subtle and widespread developmental changes in brain activation than an univariate approach.

Because psychological stress associated with fMRI scanning might confound neuroimaging findings, we examined in chapter 5 whether cortisol reactivity differed between young and old adolescents. Cortisol levels were elevated pre relative to post imaging in all participants, suggesting anticipatory stress. In addition, a subgroup of adolescents, irrespective of age, was characterized by an enhanced cortisol output during scanning. Compared with non-responders, these stress responders showed decreased activation in the inferior parietal cortex, which is related to attentional processes. Furthermore, associations between cortisol and the BOLD response during a gambling task were observed only in young as opposed to old adolescents, suggesting an age-dependent effect of cortisol on brain activation.

In chapter 6, we examined sex-related differences in brain activation during decision making in young adults aged 20-21. As previous results might be 
clouded by the use of different task paradigms, we used two decision making tasks that differed along two dimensions: motivational context, and the nature of decision making. Sex effects in brain activation were revealed during both exogenous and endogenous controlled trials of a gambling task, which had a high motivational context. There were no sex effects during the exogenous controlled gonogo task, which had a low motivational context. In two task-related areas, anterior cingulate cortex and lingual gyrus, men showed a higher BOLD response than women. In addition, task-induced deactivations seemed to be modulated by sex. These results suggest that sex differences in brain activation during decision making were related to the motivational context, rather than to the nature of decision making.

Finally, in chapter 7 we discussed the results of the empirical chapters in the context of the thesis aims. The methodological approach in this thesis of studying and controlling subject-related and methodological confounders provided new insight into the relevance of these factors for developmental fMRI results that were ignored or uncontrolled in previous studies. Based on the findings described in this thesis, functional brain maturation can be conceptualized as subtle but widespread changes in functional responsiveness of task-related voxels. Moreover, this age-distinctive pattern of brain activation seems to be independent of the specific neurocognitive processes executed, indicating a general developmental pattern. In addition, this thesis provided evidence for the importance of the factors male/female sex as well as stress reactivity to scanning on this neurocognitive development. 



\section{amenvatting}

In de adolescentie vinden grote veranderingen plaats in gedrag, neurocognitieve functies en hersenstructuur. Het is echter nog onbekend welke leeftijdsgerelateerde veranderingen in het functioneren van de hersenen ten grondslag liggen aan deze ontwikkelingen. Bovendien zijn eerdere onderzoeksresultaten naar neurocognitieve ontwikkeling mogelijk vertekend, doordat ze geen rekening houden met enerzijds proefpersoongebonden factoren, zoals geslacht, en anderzijds methodologische factoren, zoals hoofdbewegingen en de ervaren stress tijdens het hersenscan onderzoek. Het eerste doel van dit proefschrift was het onderzoeken van neurocognitieve ontwikkeling in de adolescentie en de leeftijdsgerelateerde veranderingen in hersenactiviteit die hieraan ten grondslag liggen. Om dit doel te bereiken werden twee grootschalige cross-sectionele studies uitgevoerd. We maakten hierbij onder andere gebruik van een Magnetic Resonance Imaging (MRI) scanner, waar door middel van een sterk magnetisch veld de werking van de hersenen in beeld gebracht kan worden. Verschillende leeftijdsgroepen binnen de adolescentie werden vergeleken op zowel prestaties als patronen van hersenactiviteit tijdens het uitvoeren van verscheidene cognitieve taken. Het tweede doel van dit proefschrift was het bestuderen van de invloed van proefpersoongebonden en methodologische factoren op deze ontwikkelingstrajecten. Verschillen tussen mannelijke en vrouwelijke adolescenten werden onderzocht als voornaamste proefpersoongebonden factor, en stress gevoeligheid tijdens het MRI onderzoek als belangrijkste methodologische factor. In hoofdstuk 1 staat een algemene introductie beschreven van dit proefschrift.

In hoofdstuk 2 onderzochten we het ontwikkelingspatroon van emotionele versus cognitieve functies in een groep adolescenten tussen 13 en 19 jaar. We gebruikten een mentalisatie taak, waarin proefpersonen zich bepaalde situaties moesten voorstellen en vervolgens een vraag moesten beantwoorden over emoties dan wel gedrag in deze situatie. Daarnaast gebruikten we de cognitieve Tower taak, waarin we keken naar de plan vaardigheden van proefpersonen terwijl ze ingewikkelde puzzels oplosten. De snelheid van werken op al deze drie taken verbeterde volgens een lineair verband tot in de late adolescentie. Er werden geen differentiële ontwikkelingspatronen gevonden voor emotionele versus cognitieve functies. Bovendien bleken twee proefpersoongebonden factoren van invloed op de mentalisatie snelheid, namelijk geslacht en puberteitsfase. Meisjes waren sneller dan jongens op beide mentalisatie taken. Daarnaast bleken jongens 
die verder in de puberteit waren sneller in mentalisatie dan jongens minder ver in de puberteit.

Resultaten van functionele MRI studies naar ontwikkeling suggereren dat hersenactiviteit steeds specifieker wordt en zich toespitst in focale clusters. Deze focalisatie hypothese wordt gekenmerkt door enerzijds een leeftijdsgerelateerde afname in de omvang van hersenactivaties en anderzijds een toename in signaalsterkte in taakgerelateerde gebieden. Wij onderzochten deze hypothese in hoofdstuk 3, terwijl we controleerden voor verschillende vertroebelende factoren die mogelijk de resultaten beïnvloedden richting focalisatie. Mannelijke adolescenten van respectievelijk 13, 17 en 21 jaar voerden een uitdagende goktaak uit in de scanner. Correcties voor hoofdbewegingen werden in de analyses verwerkt. Daarnaast stelden we voor elke proefpersoon afzonderlijk bepaalde clusters van activiteit vast, om zo te controleren voor de invloed van leeftijdsgerelateerde variabiliteit in hersenstructuur. Alhoewel verschillende leeftijdsgroepen binnen de adolescentie dezelfde hersengebieden activeerden tijdens het nemen van een beslissing, werd de signaalsterkte in deze gebieden beïnvloed door leeftijd. De signaalsterkte in taakgerelateerde gebieden nam toe met de leeftijd en parallel hieraan nam de deactivatie van zogenaamde standaard ('default mode') gebieden eveneens toe. Beide effecten waren sterker tijdens de moeilijke gokcondities. Deze leeftijdsgerelateerde toename in signaalsterkte ging echter noch samen met een afname in de omvang van hersenactiviteit, noch met een kwalitatieve verandering in geactiveerde gebieden. Samenvattend: we vonden enkel gedeeltelijk bewijs voor de focalisatie hypothese wanneer we corrigeerden voor vertroebelende factoren, namelijk dat de signaalsterkte toenam tussen 13 en 21 jaar in hersengebieden betrokken bij het uitvoeren van de goktaak.

In hoofdstuk 4 onderzochten we of leeftijdsgerelateerde veranderingen in hersenactiviteit verspreid door het brein plaatsvonden en tevens of dit patroon specifiek was voor een bepaalde cognitieve functie of niet. Deze kijk op functionele hersenrijping is gebaseerd op verspreide ontwikkelingseffecten die gevonden zijn in de structuur en functionele verbindingen in het brein. Genoemde aanpak contrasteert met eerdere neuroimaging studies naar ontwikkeling, waarin de nadruk lag op functionele veranderingen geassocieerd met specifieke gedragsveranderingen. De leeftijdsgerelateerde veranderingen in hersenactiviteit worden traditioneel onderzocht met een univariate analyse, waarin er per voxel (volume eenheid van de hersenen) getoetst wordt of de activiteit verschilt tussen leeftijdsgroepen. Naast deze methode gebruikten we in dit hoofdstuk ook een multivariate patroonanalyse om ontwikkeling in hersenactiviteit tijdens een simpele inhibitie taak te onderzoeken. De multivariate patroonanalyse neemt de informatie van alle voxels tegelijkertijd mee en zoekt naar een patroon van voxels die verschillen in hersenactiviteit tussen twee leeftijdsgroepen. Deze multivariate aanpak bleek zeer accuraat in het discrimineren tussen 13-jarige en respectievelijk 17- en 21-jarige proefpersonen. Dit suggereert dat functionele 
ontwikkeling verspreid door het brein plaatsvindt en dat de signaalsterkte van zowel taakspecifieke als standaard ('default mode') gebieden door leeftijd beïnvloed wordt. Bovendien toonden we aan dat dit ontwikkelingspatroon onafhankelijk is van de cognitieve functie die uitgevoerd wordt, omdat dit patroon gegeneraliseerd kan worden naar de verschillende condities binnen de gonogo taak en zelfs naar een totaal andere goktaak die dezelfde proefpersonen gedaan hadden. In tegenstelling tot de multivariate resultaten toonde de univariate analyse slechts enkele clusters aan die verschilden tussen 13- en 21-jarigen. De bevindingen van dit hoofdstuk suggereren dat een multivariate aanpak beter geschikt is voor het bestuderen van subtiele en verspreide leeftijdsgerelateerde veranderingen in hersenactiviteit dan een univariate aanpak.

Het ondergaan van een MRI scan kan stress veroorzaken, die vervolgens de functionele MRI resultaten mogelijk vertekent. In hoofdstuk 5 onderzochten we daarom of stress gevoeligheid verschilde tussen jonge en oudere adolescenten door het meten van het stresshormoon cortisol. Vóór de hersenscan waren de cortisol niveaus hoger dan erna, hetgeen kan duiden op anticiperende stress. Tevens was er een subgroep van adolescenten onafhankelijk van leeftijd, die gekenmerkt werd door een hogere cortisol output tijdens het MRI onderzoek. Vergeleken met de overige proefpersonen bleek deze stress gevoelige groep een lagere activiteit te hebben in de inferieure parietaal cortex, die betrokken is bij aandachtsprocessen. Bovendien werden associaties aangetoond tussen cortisol en hersenactiviteit tijdens een goktaak in jonge, maar niet in oudere adolescenten. Dit zou kunnen betekenen dat er een leeftijdsafhankelijk effect is van cortisol op hersenactiviteit.

In hoofdstuk 6 onderzochten we geslachtsverschillen in hersenactiviteit tijdens het nemen van beslissingen in een groep jongvolwassenen van 20-21 jaar. Omdat eerdere onderzoeksresultaten mogelijk vertroebeld zijn door het gebruik van verschillende taakparadigma's, gebruikten wij twee beslissingstaken die verschilden in motivationele context en het soort beslissing dat genomen moest worden. We gebruikten enerzijds een goktaak met een hoge motivationele context aangezien proefpersonen punten konden verdienen en geregeld feedback kregen over hun prestaties, en anderzijds een relatief simpele en saaie inhibitie taak die daardoor een lage motivationele context heeft. Daarnaast konden we binnen de goktaak onderscheid maken tussen exogene trials, waarin de juiste beslissing bepaald werd door taakfactoren, en endogene trials, waarin de juiste beslissing door de proefpersoon zelf bepaald moest worden. We vonden geslachtsgebonden verschillen in hersenactiviteit tijdens zowel exogene als endogene trials van de goktaak, terwijl we geen geslachtsverschillen vonden tijdens de inhibitietaak. Mannen activeerden twee taakgerelateerde gebieden, namelijk de anterieure cingulate cortex en gyrus linguaris, meer dan vrouwen. Daarnaast bleek de deactivatie van een aantal hersengebieden tijdens het uitvoeren van de goktaak te verschillen tussen mannelijke en vrouwelijke proefpersonen. De re- 
sultaten van dit hoofdstuk doen vermoeden dat geslachtsgebonden verschillen in hersenactiviteit tijdens het nemen van een beslissing gerelateerd zijn aan de motivationele context en niet aan het soort beslissing dat genomen moet worden.

Tenslotte, in hoofdstuk 7, bespreken we de resultaten van de onderzoekshoofdstukken in het licht van de doelen van dit proefschrift. De methodologische aanpak waarin we proefpersoongebonden en methodologische factoren controleren, heeft nieuwe inzichten verschaft over het belang van deze factoren in functioneel MRI onderzoek naar ontwikkeling. In voorgaand onderzoek werden deze vertroebelende factoren niet gecontroleerd of zelfs genegeerd. Gebaseerd op de bevindingen in dit proefschrift kunnen we functionele hersenrijping omschrijven als subtiele veranderingen in de signaalsterkte van taakrelevante hersengebieden, verspreid door het hele brein. Het is mogelijk om op basis van de patronen van hersenactiviteit tijdens neurocognitieve taken verschillende leeftijdsgroepen binnen de adolescentie accuraat van elkaar te onderscheiden. Bovendien blijkt dit onderscheidend patroon van hersenactiviteit onafhankelijk te zijn van de cognitieve functie die uitgevoerd wordt, hetgeen wijst op een algemeen ontwikkelingsprincipe. Tot slot heeft dit onderzoek aangetoond dat de factoren 'geslacht' en 'stressgevoeligheid tijdens een MRI scan' van invloed kunnen zijn op deze neurocognitieve ontwikkeling. 


\section{urriculum Vitae}

Esther Keulers werd geboren op 5 november 1980 te Geleen. Na het behalen van haar gymnasium diploma aan het Graaf Huyn College, locatie Sint-Michiel te Geleen, begon zij in 1999 haar studie Psychologie aan de Universiteit Maastricht. Ze koos als afstudeerrichting Biologische Psychologie en volgde zowel de specialisatie Ontwikkeling als Psychopathologie. Na een gecombineerde wetenschappelijke en klinische stage bij de afdeling Kinderneuropsychologie van het academisch ziekenhuis Maastricht, behaalde ze in 2004 haar diploma. In september 2004 werd Esther als onderzoeksmedewerkster aangesteld bij de afdeling Kinderneuropsychologie/Poli Leerstoornissen van het academisch ziekenhuis Maastricht, waar ze enerzijds neuropsychologische onderzoeken uitvoerde in het kader van de diagnostiek van leerstoornissen en psychiatrische stoornissen, en anderzijds betrokken was bij wetenschappelijk onderzoek binnen deze doelgroep. In januari 2007 startte zij een promotietraject bij de vakgroep Psychiatrie en Neuropsychologie van de Universiteit Maastricht. Het onderzoek uit deze periode staat beschreven in dit proefschrift. Momenteel is Esther als postdoc onderzoeker verbonden aan de afdeling Neuropsychologie en Psychofarmacologie van de Faculty of Psychology and Neuroscience van de Universiteit Maastricht. 



\section{$\perp_{\text {ublications }}$}

Keulers, E.H.H., Stiers, P., Jolles, J., 2010. Developmental changes between ages 13 and 21 years in the extent and magnitude of the BOLD response during decision making. Neuroimage, in press.

Keulers, E.H.H., Evers, E.A.T., Stiers, P., Jolles, J., 2010. Age, sex and pubertal phase influence mentalizing about emotions and actions in adolescents. Developmental Neuropsychology, 35(5), 555-569.

Keulers, E.H.H., Hendriksen, J.G.M., Feron, F.J.M., Wassenberg, R., Wuisman-Frerker, M.G.F., Jolles, J., Vles, J.S.H., 2007. Methylphenidate improves reading performance in children with attention deficit hyperactivity disorder and comorbid dyslexia: An unblinded clinical trial. European Journal of Paediatric Neurology, 11, 21-28.

Keulers, E.H.H., Hendriksen, J.G.M., Feron, F.J.M., Wassenberg, R., Boschma, L., Dings, F., Jolles, J., Vles, J.S.H., 2007. Non-verbale leerstoornissen: een spectrumstoornis met klinische implicaties. Tijdschrift voor Neuropsychologie, 2, $20-31$.

Hendriksen, J.G.M., Keulers, E.H.H., Feron, F.J.M., Wassenberg, R., Jolles, J., Vles, J.S.H., 2007. Subtypes of learning disabilities: neuropsychological and behavioural functioning of 495 children referred for multidisciplinary assessment. European Child and Adolescent Psychiatry, 16(8), 517-524.

Wassenberg, R., Hendriksen, J.G.M., Hurks, P.P.M., Feron, F.J.M., Keulers, E.H.H., Vles, J.S.H., Jolles, J., 2008. Development of inattention, impulsivity, and processing speed as measured by the d2 Test: Results of a large crosssectional study in children aged 7-13. Child Neuropsychology, 14(3), 195-210.

Keulers, E.H.H., Stiers, P., Nicolson, N.A., Jolles, J. Developmental fMRI studies: should cortisol reactivity be considered as confounder? Under review.

Keulers, E.H.H., Stiers, P., Jolles, J. Sex differences in brain activation are related to motivational context rather than to the nature of decision making. Under review. 